\title{
Development of Electrochemical Microsensors for in vivo Neurotransmitter Detection
}

\author{
Cheng Yang \\ Jinan, Shandong, China \\ B.S. East Tennessee State University, 2012
}

A Dissertation presented to the Graduate faculty of the University of Virginia in Candidacy for the Degree of Doctor of Philosophy

Department of Chemistry

University of Virginia

November, 2016 


\section{Abstract}

Everything that happens in our body and all the interactions we have with the outside world are controlled by our brain. Studies of neurotransmitters are critical for a better understanding of how our brain works. Fast-scan cyclic voltammetry (FSCV) is the most popular electrochemical technique for the in vivo detection of electroactive neurotransmitters and neurochemicals with high temporal resolution. To further improve the detection selectivity, sensitivity, and spatial resolution, carbon nanomaterial based microelectrodes are applied to enhance FSCV for the neurotransmitters detection. In this thesis, I introduce and discuss synthesis and fabrication of several novel carbon nanomaterials, the effect of different surface modifications, and their applications for in vivo neurotransmitter detection.

Chapter 1 covers the recent advances in carbon nanomaterial based electrochemical sensors for direct neurotransmitter detection. First, strategies are compared to incorporate carbon nanomaterials into electrochemical sensors for neurotransmitter detection. Second, several new applications are highlighted as well as studies to address the remaining challenges for implementation. Chapters 2-5 describe new carbon nanomaterials and surface treatments for enhanced neurotransmitter detection. The direct growth of CNTs on metal wires is introduced in Chapter 2. Chapter 3 and Chapter 4 introduce three surface modification methods, laser treatment, $\mathrm{O}_{2}$ plasma etching, and anti-static gun treatment, to improve sensitivity, selectivity, and bio-fouling properties on CNT yarn microelectrodes. In Chapter 5, three different types of CNT fiber materials, CNT yarn, PEI/CNT fiber, and CA/CNT fiber, are introduced and compared for the detection of neurotransmitters. Surface physical properties and the electrochemical performance to neurotransmitters are characterized in these studies and correlated using Langmuir adsorption isotherm and diffusion profiles. Moreover, in vivo measurements are performed for dopamine at CNT-Nb microelectrodes and laser treated CNT yarn microelectrodes in Chapter 2 and Chapter 3. The effect of bio-fouling on CNT yarn is discussed in Chapter 4. 
Chapter 6 and Chapter 7 introduce two novel electrode fabrication methods based on 3D printing: a 3D printed mold assisted microelectrode fabrication method and a novel 3D printed electrode design for fiber-like materials. The 3D printing technology with low-cost, high efficiency, and customizable design provides a new path to apply our studies on novel fiber-like carbon nanomaterials and surface modifications to broader applications.

Overall, this thesis explores the synthesis and fabrication of several novel carbon nanomaterials, the effect of different surface modifications, and their applications for in vivo neurotransmitters detection. Moreover, the electrode surface properties are also characterized and then correlated to their electrochemical performance. The systematic comparison of different carbon nanomaterials and different surface modifications provides a useful structure to evaluate which new nanomaterials would be good as electrochemical sensor and which electrode a neuroscientist might choose for different experiments. 


\section{Table of Contents}

Abstract

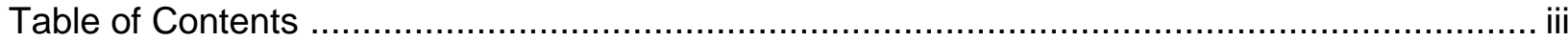

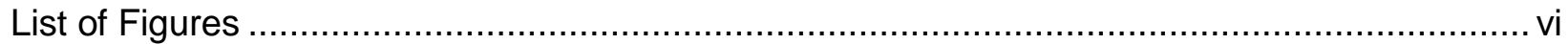

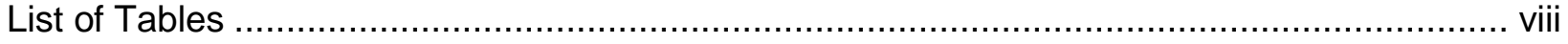

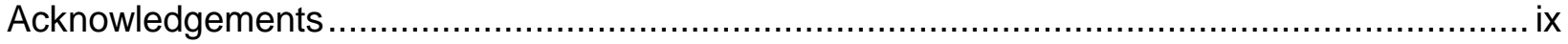

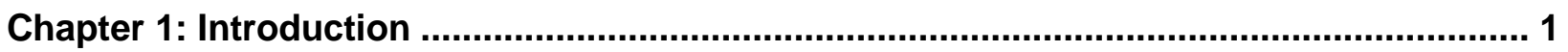

1.1 Electrochemical Detection of Neurotransmission ..................................................... 2

1.2 Basic Electrochemistry and Fast Scan Cyclic Voltammetry ……….............................. 3

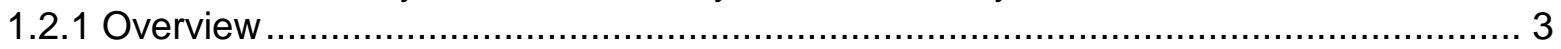

1.2.2 Fast-Scan Cyclic Voltammetry ..................................................................... 4

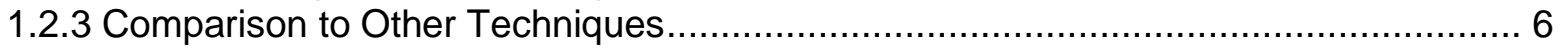

1.3 Carbon Nanomaterial Based Microelectrodes for Neurotransmitter Detection ................... 8

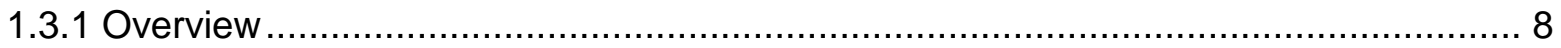

1.3.2 Carbon Nanomaterial based Electrodes Using Dip Coating/Drop Casting Method ....10

1.3.3 Direct Growth of Carbon Nanomaterials on Electrode Substrates ...........................13

1.3.4 Carbon Nanotube-Fiber Microelectrodes.....................................................15

1.3.5 Carbon Nanoelectrodes and Carbon Nanomaterial Based Electrode Array ...............17

1.3.6 3D-Printing Technologies for Carbon Nanomaterial based Microsensors Fabrication20

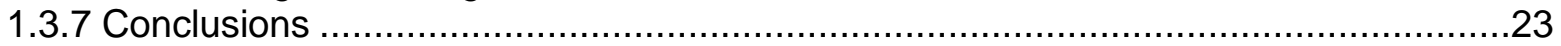

1.4 Challenges and Future Directions.................................................................24

1.4.1 Correlation between Electrochemical Performance and Carbon Nanomaterial Surface

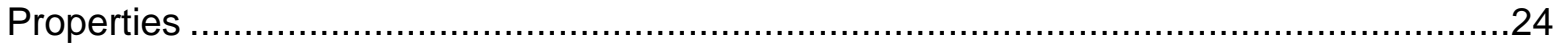

1.4.2 Effect of Bio-Fouling on Carbon Nanomaterial based Microelectrodes for in vivo

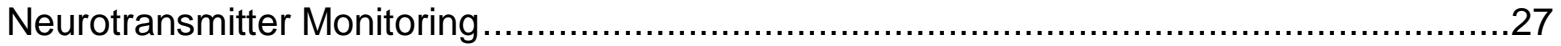

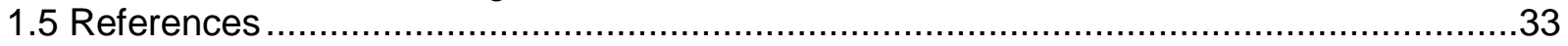

Chapter 2: Carbon Nanotubes Grown on Metal Microelectrodes for the Detection of

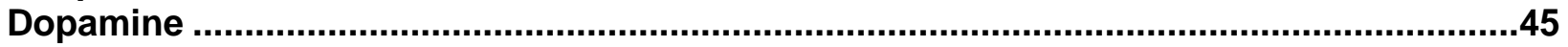

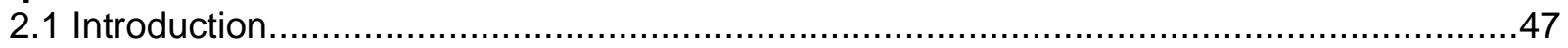

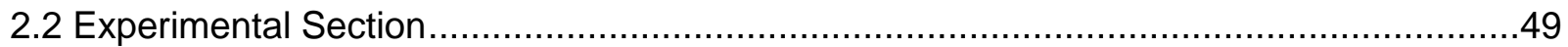

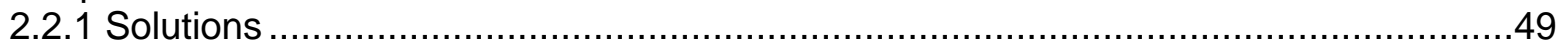

2.2.2 Synthesis of CNTs coated metal wires and carbon fibers ...................................49

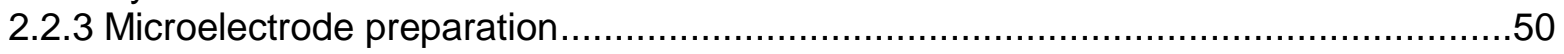

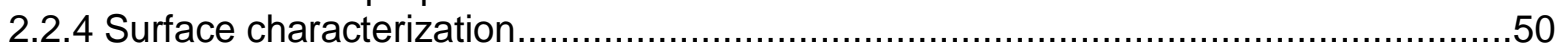

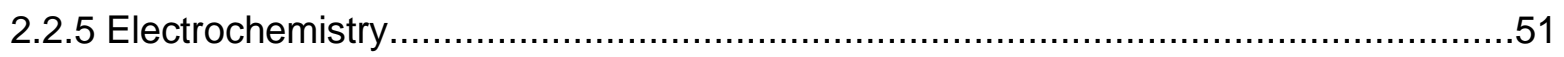

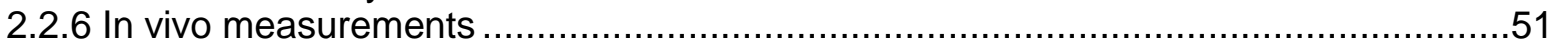

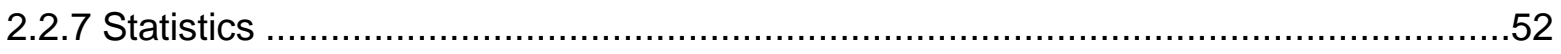

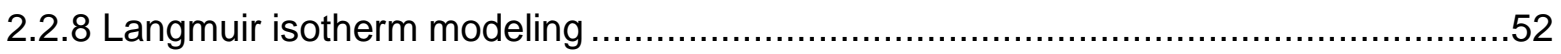

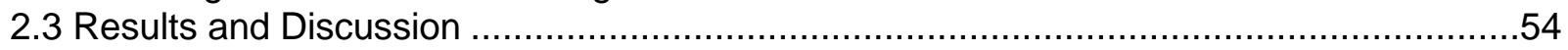

2.3.1 Characterization of CNTs grown on metal wires and carbon fibers .........................54

2.3.2 FSCV of dopamine at bare metal wire microelectrodes and CFMEs ........................56

2.3.3 FSCV of dopamine at CNT-metal wire microelectrodes and CNT-CFMEs.................57

2.3.4 Characterization of other metal substrates for CNT growth ..................................62

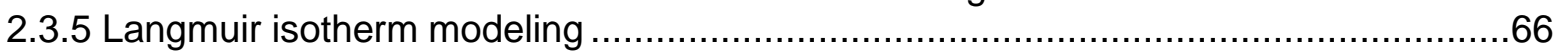

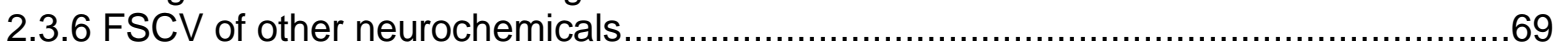

2.3.7 In vivo detection of dopamine at CNT-Nb microelectrodes .....................................

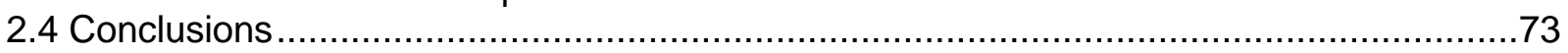

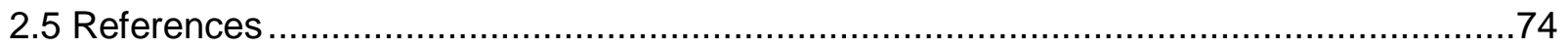


Chapter 3: Laser Treated Carbon Nanotube Yarn Microelectrodes for Rapid and Sensitive

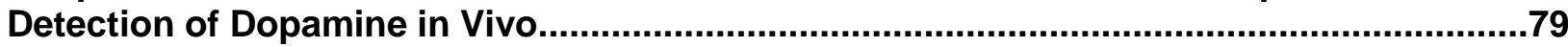

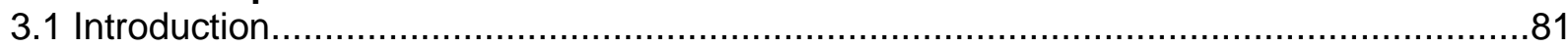

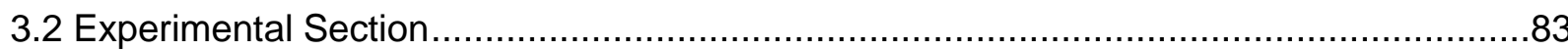

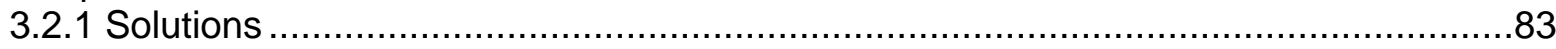

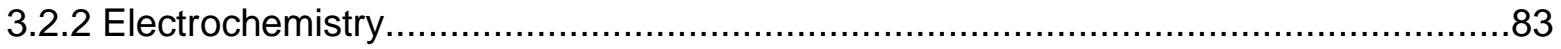

3.2.3 Carbon Nanotube Yarn Microelectrode Preparation .............................................8

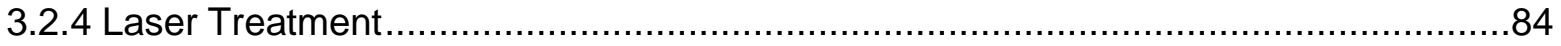

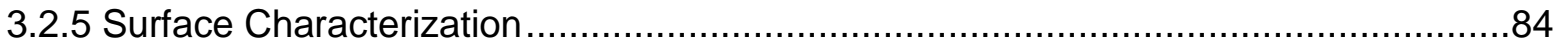

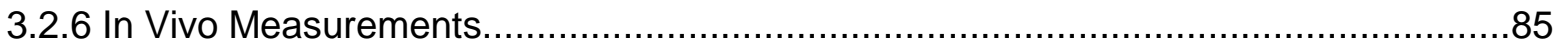

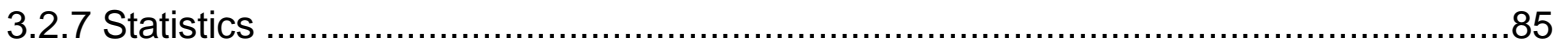

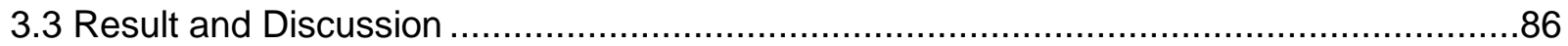

3.3.1 Laser Treatment Improves Carbon Nanotube Yarn Microelectrode Signals ...............86

3.3.2 Improved Temporal Resolution for Dopamine Detection at Laser-Treated Carbon

Nanotube Yarn Microelectrodes ..............................................................................90

3.3.3 Surface Characterization of Laser-Treated Carbon Nanotube Yarn Microelectrode ..92

3.3.4 Correlation of Electrochemical and Surface Properties ........................................99

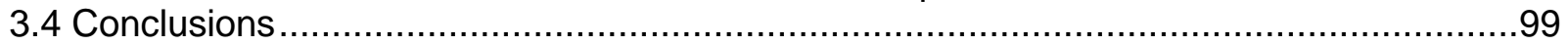

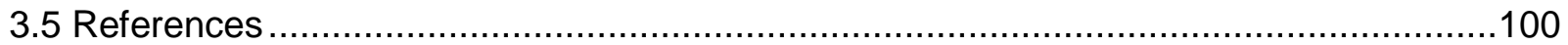

Chapter 4: $\mathrm{O}_{2}$ Plasma Etching and Anti-Static Gun Surface Modifications for CNT Yarn Microelectrode Improve Sensitivity and Anti-Fouling Properties....................................103

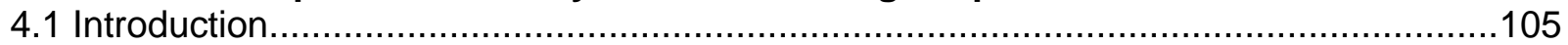

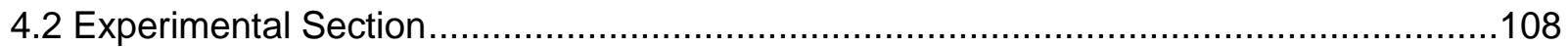

4.2.1 Carbon Nanotube Yarn Microelectrode Preparation ........................................108

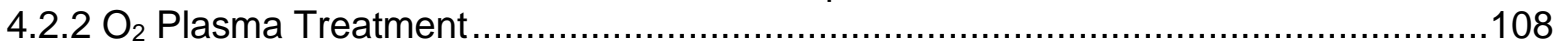

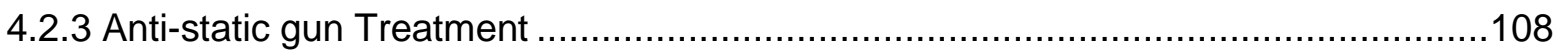

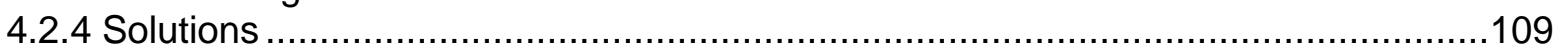

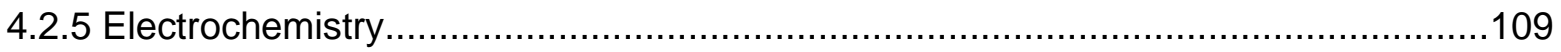

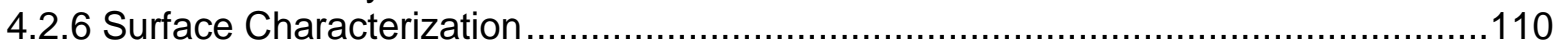

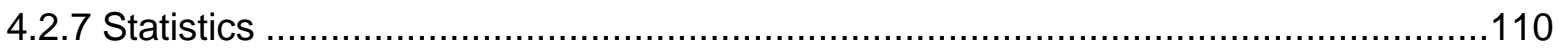

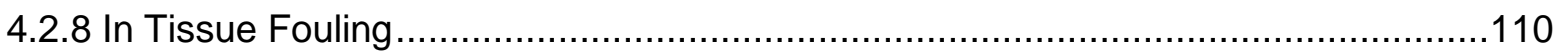

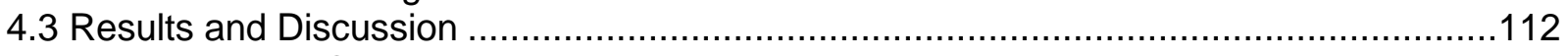

4.3.1 Parameter Optimization...........................................................................112

4.3.2 Comparison of Electrochemical Properties of $\mathrm{O}_{2}$ plasma etched and Anti-Static Gun

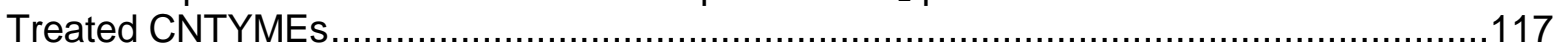

4.3.3 Physical Characterization of Treated CNTYMEs ……....................................120

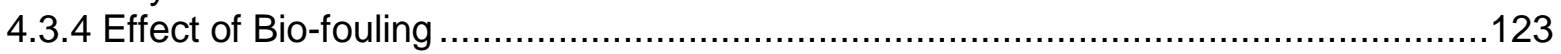

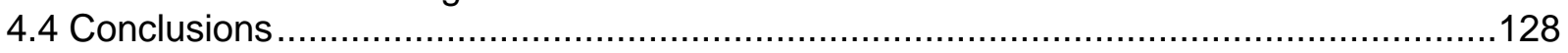

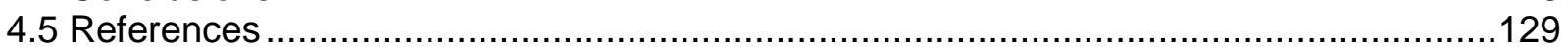

Chapter 5: Evaluation of Carbon Nanotube Fiber Microelectrodes for Neurotransmitter

Detection: Correlation of Electrochemical Performance and Surface Properties .............133

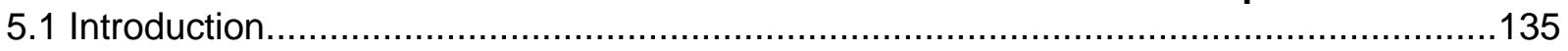

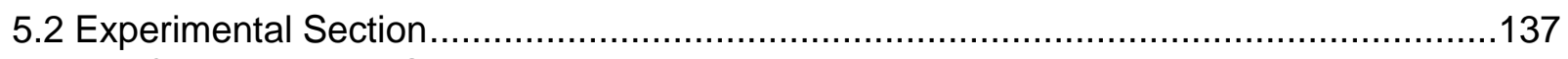

5.2.1 Chemicals and Solutions .............................................................................137

5.2.2 PEI/CNT Fiber and CA/CNT Fiber Preparation ...............................................137

5.2.3 CNT Fiber and Yarn Microelectrode Fabrication ............................................137

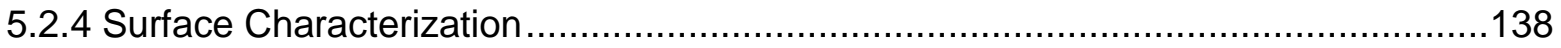

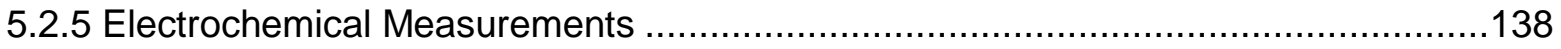

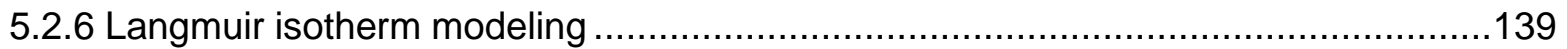

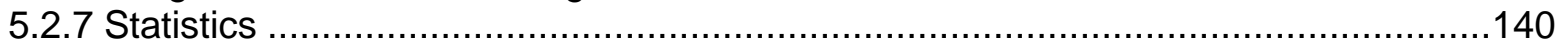

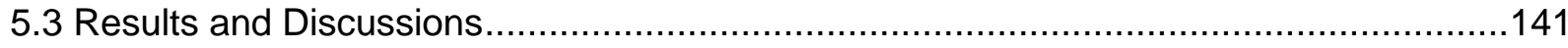


5.3.1 Surface Comparison of CNT Fiber Microelectrodes

5.3.2 Electrochemical Characterization of Dopamine Detection at CNT Fiber

Microelectrodes.

5.3.3 Electrochemical Characterization of Other Neurochemicals at CNT Fiber

Microelectrodes.

5.3.4 Dopamine Sensitivity at Rapid Scan Repetition Frequency ...............................148

5.3.5 Correlation of Electrochemical Performance and Surface Properties .....................150

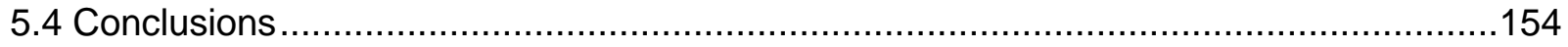

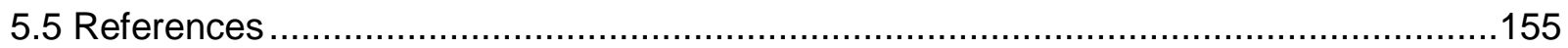

Chapter 6: Novel Carbon-Fiber Microelectrode Batch Fabrication using a 3D-Printed Mold and Polyimide Resin .....................................................................................................158

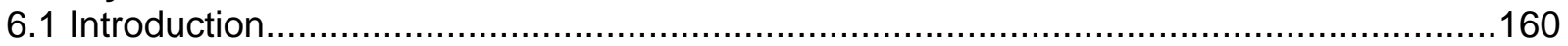

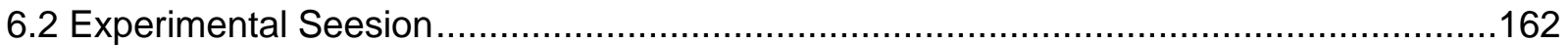

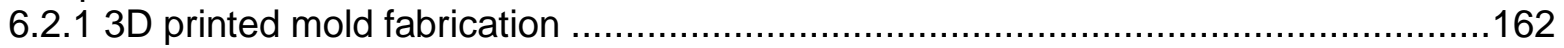

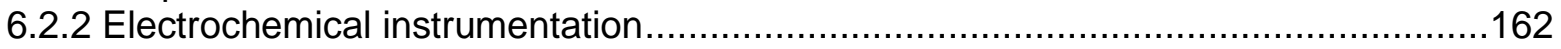

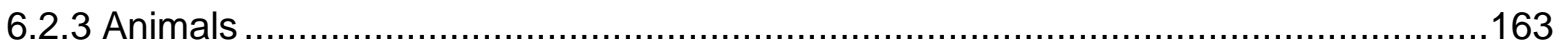

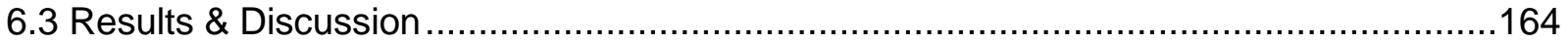

6.3.1 Polyimide-Insulated Microelectrode Fabrication Using 3D-Printed Mold ..................164

6.3.2 Electrochemical Characterization in Vitro and in Vivo........................................166

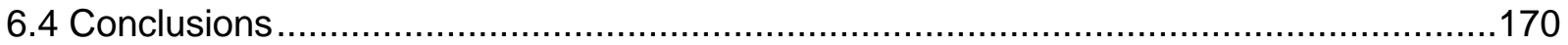

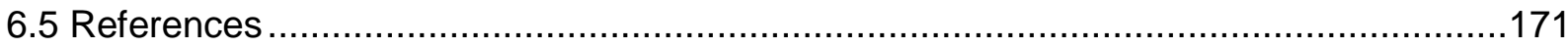

Chapter 7: High Performance, Low Cost Carbon Nanotube Yarn based 3D Printed Electrodes Compatible with a Conventional Screen Printed Electrode System................173

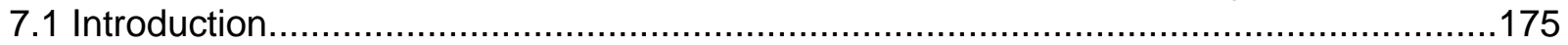

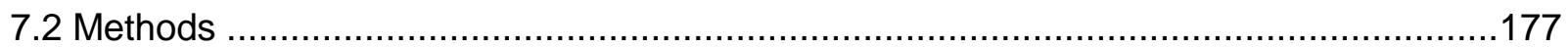

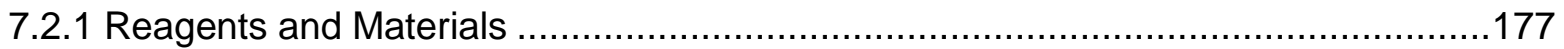

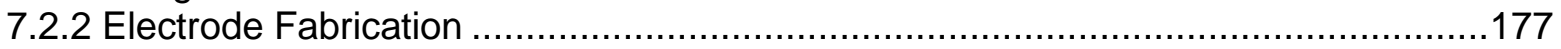

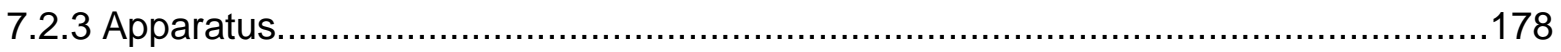

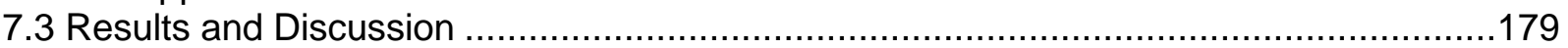

7.3.1 CNT Yarn based 3D Printed Electrode Fabrication ........................................179

7.3.2 Electrochemical Characterizations ................................................................181

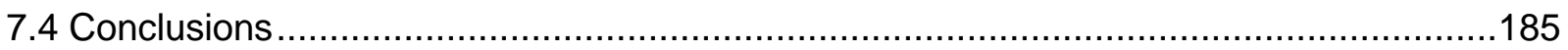

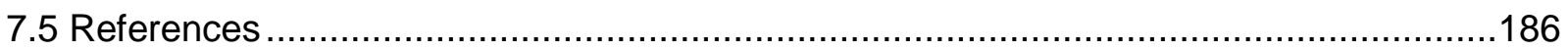

Chapter 8: Conclusions and Future Directions .......................................................189

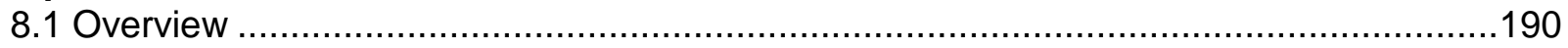

8.1.1 Evaluation of Novel Carbon Nanomaterials and Different Surface Modifications for in

vivo Neurotransmitter Monitoring ............................................................................190

8.1.2 Correlation of Surface Properties to Electrochemical Performance for

Neurotransmitters Detection............................................................................191

8.1.3 3D Printing Technology Assisted Carbon Electrode Fabrication and the Application for

Electrochemical Sensing ..................................................................................192

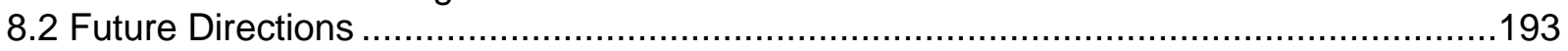

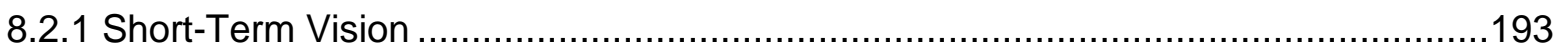

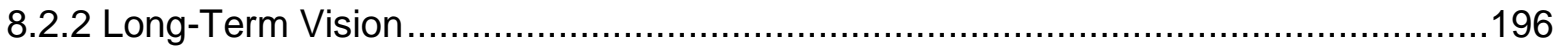

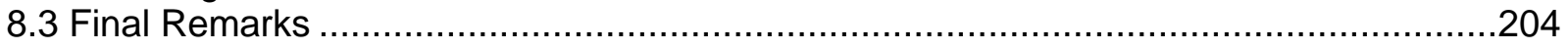

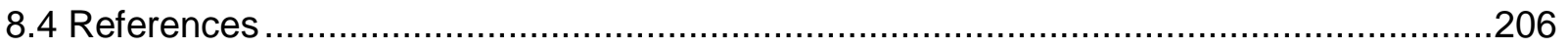




\section{List of Figures}

Figure 1.1: Rapid measurement of dopamine using FSCV ....................................................

Figure 1.2: CNT yarn microelectrodes ................................................................................ 17

Figure 1.3: Nanoelectrode designs for neurotransmitter detection .......................................... 18

Figure 1.4: Schematic illustration of amperometric response at the electrode/electrolyte interface in different concentrations of protein .............................................................................. 31

Figure 2.1: SEM images of bare metals and metals with CNT growth .........................................5 54

Figure 2.2: Raman spectrum of CNT grown on different metal substrates ...................................56

Figure 2.3: Electrochemical response of bare metal or carbon fibers ........................................57

Figure 2.4: Comparison of electrochemical response at CNT-grown niobium, tantalum and

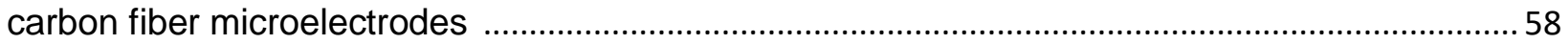

Figure 2.5: Effect of equilibration at CNT-metal microelectrodes..............................................59

Figure 2.6: CNT-Nb microelectrodes stability experiment .......................................................... 60

Figure 2.7: SEM images of bare Mo, W, Pd, SS, and Ti, and same substrates after CNT growth

Figure 2.8: Electrochemical response of bare metals

Figure 2.9: Comparison of the electrochemical response at CNT-grown metals microelectrodes

Figure 2.10: Plot of normalized anodic current to corresponding dopamine concentration .........67

Figure 2.11: Plot of normalized cathodic current to corresponding dopamine concentration .....68

Figure 2.12: Detection of other neurochemicals at CNT-Nb microelectrodes ...............................71

Figure 2.13: Dopamine detection in vivo at CNT-Nb microelectrodes .......................................72

Figure 3.1: Comparison of the response to $1 \mu \mathrm{M}$ dopamine at an unmodified disk CNTYME and

the same electrode after 15 pulses laser treatment...................................................................... 86

Figure 3.2: Stability test at laser treated CNTYMEs ……………………………….................. 89

Figure 3.3: Example cyclic voltammograms and comparison of $200 \mu \mathrm{M}$ ascorbic acid on an unmodified CNTYME and a laser-treated CNTYME ................................................................. 90

Figure 3.4: Effect of scan repetition frequency for $1 \mu \mathrm{M}$ dopamine detection at CFMEs, unmodified and laser treated CNTYMEs ……………................................................................ 91

Figure 3.5: Comparison of the effect of FSCV scan repetition frequency in vivo ......................... 92

Figure 3.6: SEM images of unmodified CNTYME and laser treated CNTYME ........................... 93 Figure 3.7: Representative three-dimensional laser confocal profile image of CFME, unmodified CNTYME, and the same microelectrode after laser treatment .................................................. 95 Figure 4.1: Comparison of the response to $1 \mu \mathrm{M}$ dopamine at an unmodified disk CNTYME and the same electrode after treatment of 1 min and 3 mins $\mathrm{O}_{2}$ plasma etching. 113

Figure 4.2: SEM images of an unmodified CNTYME and the same electrode after $1 \mathrm{~min}$ and 3 mins $\mathrm{O}_{2}$ plasma etching

Figure 4.3: Comparison of the response to $1 \mu \mathrm{M}$ dopamine at an unmodified disk CNTYME and the same electrode after treatment of 1 trigger, 2 triggers, and 4 triggers of anti-static gun modifications

Figure 4.4: SEM images of an unmodified CNTYME and the same electrode after anti-static gun treatments with 1 trigger, 2 triggers, and 4 triggers

Figure 4.5: Oxidation current change with time at anti-static gun treated CNTYMEs with 2 triggers

Figure 4.6: Raman spectra obtained at CNTYMEs with different surface modifications .......... 122

Figure 4.7: Comparison of the response to $1 \mu \mathrm{M}$ dopamine and background current at an unmodified CFME and the same electrode after different triggers of anti-static gun treatment 123 
Figure 4.8: Comparison of the response to $1 \mu \mathrm{M}$ dopamine before and after $2 \mathrm{H}$ in-tissue fouling, at an unmodified CNTYME, $\mathrm{O}_{2}$ plasma etched CNTYME, and anti-static gun treated CNTYMEs.

Figure 5.1: Surfaces of microelectrodes.

Figure 5.2: Background subtracted cyclic voltammograms and current versus time trace plot to $1 \mu \mathrm{M}$ dopamine at CA/CNT fiber, PEI/CNT fiber, and CNT yarn microelectrodes 145

Figure 5.3: Detection of different neurochemicals at CNT fiber microelectrodes 148

Figure 5.4: Effect of scan repetition frequency for $1 \mu \mathrm{M}$ dopamine detection 149

Figure 5.5: Langmuir isotherm plots obtained at different CNT fiber microelectrodes 151

Figure 6.1: 3D-printed mold design and polyimide-insulated carbon fiber microelectrodes fabrication process

Figure 6.2: Electrochemical characterization of polyimide-insulated CFMEs

Figure 6.3: Dopamine detection in vivo at polyimide-insulated CFMEs 169

Figure 7.1: Illustration of electrode design and CNT yarn 3D Printed Electrode 180

Figure 7.2: Electrochemical characterization of CNT yarn 3D printed electrodes 182

Figure 8.1: Number of publications for dopamine analysis using CNT and graphene based electrochemical methods (from 2006 to 2015)

Figure 8.2: SEM images of ion polished CNT yarns

Figure 8.3: CVs recorded at an open tube CNPE in $10 \mu \mathrm{M}$ dopamine solution with different equilibration time and dopamine oxidation scheme 


\section{List of Tables}

Table 2.1: Average $\Delta \mathrm{E}_{p}$, current density, and limit of detection for $1 \mu \mathrm{M}$ dopamine at CNT-grown microelectrodes and CFMEs

Table 2.2: Average equilibrium constants and adsorption Gibbs free energy for dopamine and

DOQ at CNT-grown microelectrodes and CFMEs

Table 3.1: Comparison of dopamine detection at CNTYMEs and laser treated CNTYMEs........87

Table 3.2: Surface properties of CNTYMEs …………........................................................... 94

Table 4.1: Comparison of dopamine detection at anti-static gun treated CNTYMEs with different triggers 115

Table 4.2: Comparison of dopamine detection at unmodified and $\mathrm{O}_{2}$ plasma etched CNTYMEs 118

Table 4.3: Comparison of dopamine detection at unmodified and anti-static gun treated CNTYMEs. 119

Table 4.4: Surface properties of CNTYMEs with different surface modifications 121

Table 4.5: Comparison of dopamine detection at CNTYMEs before/after in tissue fouling........ 125

Table 5.1: Elemental Contents at the tips of Microelectrodes Measured by EDS ...................... 142

Table 5.2: Comparison of Electrochemical Dopamine Detection 144

Table 5.3: Average Oxidation Current Density for Different Neurochemicals at CA/CNT Fiber, PEI/CNT Fiber, and CNT Yarn Microelectrodes 146

Table 5.4: Average Equilibrium Constants ( $\beta$ ) for Dopamine and Dopamine-o-quinone 153 


\section{Acknowledgements}

First, I would like to express my sincere gratitude to my advisor, Dr. Jill Venton, for the continuous support of my Ph.D study and related researches, for her patience and large amount of time of editing and shaping my writings. I have nothing but the highest regard for her: I could not have imagined having a better advisor and mentor like her for my Ph.D study. I would also like to thank everyone in the lab for being supportive. I could not finish my Ph.D study without their huge help. My sincere thanks goes to Dr. Christopher B. Jacobs for being my mentor, being extremely patient in training me, giving me numerous suggestions on how to become a good scientist, and continuous help on my research even after his moving to Oak Ridge. Dr. Jacobs also helped synthesizing CNTs-metal sample and taking TEM images in Chapter 2, performing electrochemical impedance spectroscopy measurement on CNT yarn and fibers in Chapter 5. Big thanks to Elefterios Trikantzopoulos for synthesizing CNT fibers in Chapter 5, help fabricating electrodes and writing in Chapter 5 and 6. Thank you to Dr. Michael Nguyen, Dr. Mallikarjunarao Ganesana, Ying Wang, and Scott Lee for performing animal experiments for me in Chapter 2, Chapter 3, Chapter 4, and Chapter 6. I would also like to thank Dr. Ning Xiao for training me when I first came to the lab, Dr. Alexandros Zestos for his help, and great thanks to Richard White for training me to use scanning electron microscopy and Energy-dispersive X-ray spectroscopy at UVa, and helping me making the cylindrical CNT yarn using ion polisher in Chapter 8 . Thanks to my committee members of my dissertation, Dr. Ian Harrison, Dr. James Demas, Dr. Cameron Mura, and Dr. Nathan Swami, for giving me the opportunity to present my work and valuable suggestions on my dissertation.

I would also like to thank all of our collaborators. At the Center for Nanophase Materials Sciences, Oak Ridge National Laboratory, I appreciate David B. Geohegan for giving us the opportunity to use facilities at CNMS and work together with CNMS experts in material science, Dr. Alexander A Puretzky for hosting us in the user program and great help on synthesizing CNTs- 
metal in Chapter 2, Dr. Ilia N. Ivanov for performing the laser confocal surface roughness measurement in Chapter 3 and Chapter 4, Dr. Dale K. Hensley for training me SEM, EDS, and synthesizing samples, and Dr. Dayrl P. Briggs for training me how to work in clean room and how to use ALD. Thanks also goes to Dr. Michael V. Mirkin and his graduate student Keke Hu at City University of New York for collaborating and providing us carbon nanopipettes, Dr. Kateri DuBay and her graduate student Qizhang Jia at UVa for working together on the diffusion and adsorption modeling of neurotransmitters at carbon nanomaterial electrode surface, as well as Dr. Liangbin Hu and his graduate student Yanan Chen for proving graphene oxide samples.

Finally I want to thank my family. To my parents, Dong Yang and Jing Du, for bring me to this world, supporting their love and support throughout my life, and backing me up when I am down. You sacrificed your own happiness sending me abroad and giving me the strength to chase my dreams. It may take a lifetime, but I'll do everything to repay for what you have done for me. I am and will forever be grateful of you. I would also like to thank my beautiful and considerate wife, Mimi Zhu. It is my great luck to meet you at UVa and fall in love with you. Thanks for having answers to all my questions. I cannot finish my Ph.D study without her support, encouragement, patience and unwavering love. Last but most important, I would like to give my deepest gratitude to my wife for breeding our unborn baby. Dear baby, I cannot wait to meet you. 
Chapter 1: Introduction 


\subsection{Electrochemical Detection of Neurotransmission}

Brain neuronal communication occurs primarily through the exocytotic release of neurotransmitters into synaptic junctions between two neurons and the surrounding extracellular fluid. Neurotransmitters synthesized in the axon are packed into specialized synaptic vesicles. The typical view of neurotransmission is the diffusion of neurotransmitters across the synapse, binding to dendritic receptors, and activating further signaling pathways. ${ }^{1}$ Many debilitating disorders such as Parkinson's disease, Alzheimer's disease, depression, and drug addiction occur due to problems with neurotransmission. ${ }^{2,3}$

Dopamine (3,4-dihydroxyphenethylamine, DA), a catecholamine, modulates many aspects of brain circuitry and is implicated in several neurological diseases, including Parkinson disease, which is also released from the synapse. ${ }^{4}$ Dopamine is also a standard analyte to evaluate the neurotransmitters detection at electrodes. The basal levels of extracellular dopamine released from the synapse are around 0.01-0.03 $\mu \mathrm{M}$, while phasic release during a burst of neuronal firing can be $0.1-1 \mu \mathrm{M} .^{5}$ The low extracellular concentration requires high detection sensitivity of the measuring device. Moreover, since the extracellular neurotransmitter concentrations change rapidly, ${ }^{5}$ real-time methods for neurotransmitter detection are necessary to understand the dynamics of neurotransmission and develop better treatments for these diseases.

Electroanalytical techniques have been widely developed and applied to investigate electroactive neurotransmitters and their metabolites. Such techniques have led to a better understanding of neurochemicals events ranging from exocytosis of isolated cells to in vivo neurotransmission. ${ }^{6,7}$ Electrochemical sensors are currently the gold standard for the rapid detection of neurotransmitters through their redox reactions. The advantages of electrochemical neurotransmitters sensing include fast response, high sensitivity and selectivity, as well as high temporal and spatial resolution. As to the direct detection with electrochemical methods, target 
molecules need to be electroactive within the potential window of the interstitial fluid and common targets include biogenic amines and their metabolites, as well as uric acid and ascorbic acid. Using enzyme modified electrodes, many other non-electroactive neurotransmitters can be detected in vivo as well. ${ }^{8}$

\subsection{Basic Electrochemistry and Fast Scan Cyclic Voltammetry}

\subsubsection{Overview}

Electrochemical sensing concepts for neurotransmitters are currently the gold standard, and several reviews have been published summarizing related works. ${ }^{5,9-18}$ To monitor neurotransmitters fluctuations in living tissues, several different electrochemical techniques have been applied such as amperometry, potential pulse methods, and cyclic voltammetry. ${ }^{19,20}$ Generally, electrochemical methods detect target neurotransmitters through their redox reaction at a solid electrode. When a potential is applied to the electrode, two electrochemical processes occur at the electrode surface: Faradic and non-Faradic processes. In the Faradic process, target analyte redox reaction causes electron transfer, which is proportional to the amount of the chemicals reacted. Thus, the moles of analytes which react can be calculated based on the Faradic current. The currents generated provide a quantitative measurement of dynamic chemical fluctuations that can be correlated to pharmacology, behavior, and disease pathology. Target molecules need to be electroactive within the potential window of the interstitial fluid; these include the biogenic amines (dopamine, norepinephrine, and serotonin, etc.) and their metabolites. Processes such as changes in the electrode surface morphology and electron-solution interface structure can cause current changes as well, but no actual electron exchanges happens at the electrode-solution interface. The current induced in this process is called non-Faradic current, in which a charge gradient is created and rearranged at the interface till the equilibrium is reached. 
The charging current, usually called double layer charging, is proportional to both of the scan rate and the capacitance of the electrode (related to surface area).

\subsubsection{Fast-Scan Cyclic Voltammetry}

In cyclic voltammetry, a triangle waveform is applied to an electrode to oxidize and reduce electroactive species at electrode surface. From the cyclic voltammogram, the Faradic currents can be used to quantitatively calculate the analytes concentration. Moreover, compared to constant-potential amperometry, both of the oxidation and reduction peak potentials can be used as fingerprints to distinguish different analytes from the mixture, which makes cyclic voltammetry able to detect multiple electroactive neurotransmitter and neurochemicals simultaneously. Sensitivity, selectivity, and temporal resolution can be further optimized by altering the potential limits, scan rate, and application frequency of waveform.

Fast-scan cyclic voltammetry (FSCV) is the most popular and widely used electrochemical technique in the field of neuroscience for the in vivo measurement of neurotransmitters and neurochemicals. Compared to conventionally used cyclic voltammetry methods, FSCV provides an extremely fast scan rate $(400-2000 \mathrm{~V} / \mathrm{s})$, which allows the scan repetition frequency as fast as $500 \mathrm{~Hz}$ to follow rapid biological process. Figure 1.1A shows the typical triangle waveform applied for the detection of dopamine. The potential is ramped from -0.4 to $1.3 \mathrm{~V}(\mathrm{vs} \mathrm{Ag} / \mathrm{AgCl})$ and back at $400 \mathrm{~V} / \mathrm{s}$ with the scan repetition rate of $10 \mathrm{~Hz}$. Therefore, the entire triangle waveform takes approximately $8.5 \mathrm{~ms}$ and is repeated every $100 \mathrm{~ms}$. For the rest of time when the triangle waveform is not applied, the electrode is held at the holding potential $(-0.4 \mathrm{~V})$ between scans, allowing cation such as dopamine under philological pH to adsorb on the electrode surface, which helps enhancing the detection sensitivity. For example, at conventionally used carbon fiber microelectrodes (CFMEs), the longer duration at the holding potential between scans, the larger dopamine current detected..$^{21,22}$ 
A.

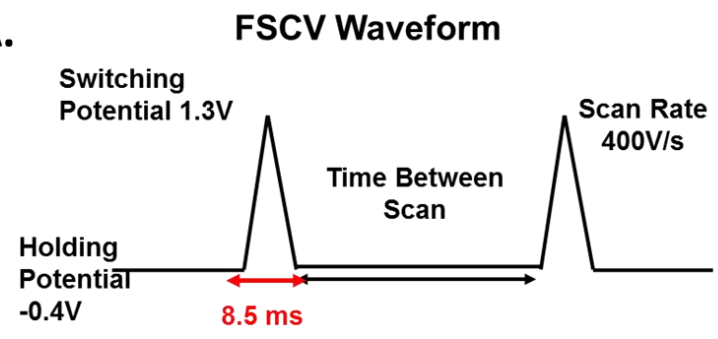

C.

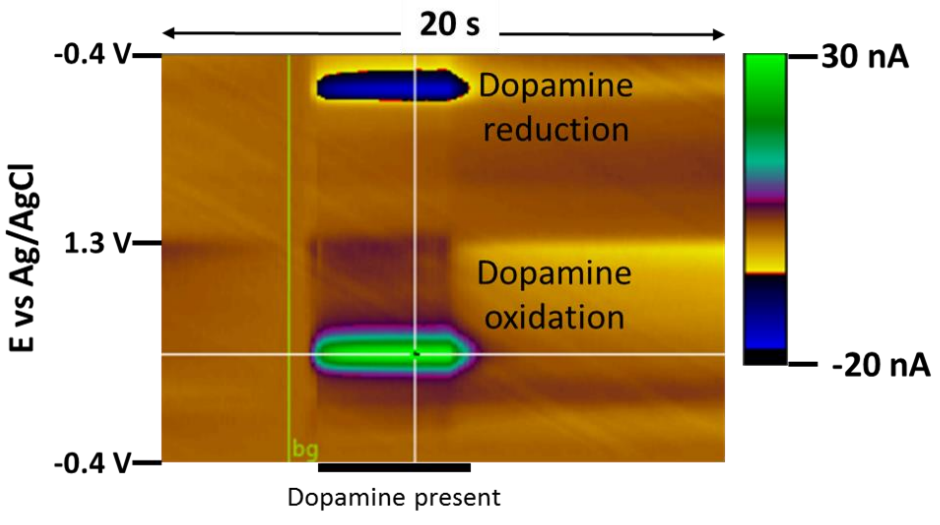

B. Background Current

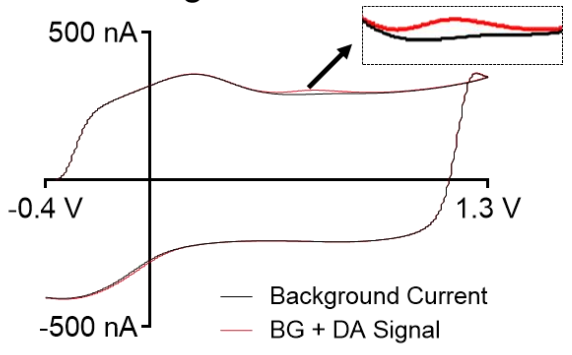

Cyclic Voltammogram

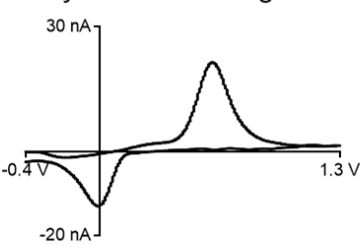

Oxidation Current vs Time

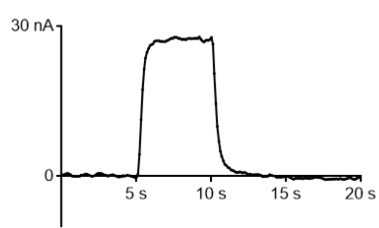

Figure 1.1: Rapid measurement of dopamine using FSCV. (A) A typical FSCV triangle waveform for dopamine detection. The potential is ramped from the holding potential $(-0.4 \mathrm{~V}$ for typical dopamine detection) to the switching potential (typically $1.3 \mathrm{~V}$ ) at a certain scan rate $(400 \mathrm{~V} / \mathrm{s})$, and then reversed back to the holding potential at the scan rate. The scan repetition rate is usually applied at $10 \mathrm{~Hz}$, but could increase up to $110 \mathrm{~Hz}$ with the scan rate of $400 \mathrm{~V} / \mathrm{s}$, and $500 \mathrm{~Hz}$ with $2000 \mathrm{~V} / \mathrm{s}$ theoretically. (B) Background current detected by FSCV with the triangle waveform shown in A applied. The black line is collected in phosphate-buffered saline (PBS) buffer solution, and red line is collected in PBS buffer with the presence of $1 \mu \mathrm{M}$ dopamine. Inset shows a zoomin view of dopamine oxidation current. (C) Example of FSCV data to $1 \mu \mathrm{M}$ dopamine in PBS buffer. Color plot showing dopamine detection in a flow-cell experiment, with X-axis referring to time, $\mathrm{Y}$ axis is voltage, and the pseudo color refers to current. Vertical white slicing line gives the background subtracted cyclic voltammetry. And horizontal slicing line gives the oxidation current vs. time plot, in which PBS buffer solution flows past the electrode initially and then switched to dopamine solution at $5 \mathrm{~s}$ (black bar). Current drops back to baseline once dopamine is removed at $10 \mathrm{~s}$.

The double layer charging current appears on the electrode surface when a potential is applied, which is proportional to the scan rate. Since the scan rate is extremely fast using FSCV, a large background charging current is produced, and the oxidation and reduction currents of the 
electroactive analyte, such as dopamine, only make a small increase in the current. In addition, the background charging current of the electrode is also proportional to surface area and is expected to increase with nanostructuring. ${ }^{5}$ Background current is conventionally digitally subtracted from the background current several seconds before the target cyclic voltammogram, leaving only the currents caused by redox reaction of the analytes.

A false color plot is used to view the entire data set collected by FSCV, which provides rich information such as currents and voltages changes with time. Voltage is plotted on the y-axis, time is shown on the x-axis, and current is represented in pseudo color. As shown in the example dopamine cyclic voltammogram (Figure 1.1C), a green area centered at around $600 \mathrm{mV}$ is due to the dopamine oxidation to form dopamine-o-quinone, and a blue area centered at around -200 $\mathrm{mV}$ indicates the reduction of dopamine-o-quinone back to dopamine. If a horizontal slice of the color plot at any given potential is taken, the oxidation current change over time (dynamic curve) can be obtained. This plot is usually taken at the oxidation peak of the analyte, which can be used to characterize the speed of electrode response (temporal response). A cyclic voltammogram can be observed by taking a vertical slice of the color plot, showing the change of current with potentials. Peak currents after background subtraction are used to calculate the analyte concentration based on Faraday's law. The separation between the oxidation and reduction peaks, $\Delta \mathrm{E}_{\mathrm{p}}$, is a qualitative measure of the analytes electron transfer kinetics with electrode surface. The application of carbon nanomaterial or further surface modifications are expected to cause peaks shift and reduce $\Delta \mathrm{E}_{\mathrm{p} .}{ }^{18,23-25}$

\subsubsection{Comparison to Other Techniques}

FSCV has become the most popular electrochemical technique for the detection of neurotransmitters and neurochemicals because of its wide range of electroactive target analytes, real-time measurement ability, and high selectivity. FSCV not only has the natural high-selectivity 
to distinguish different analytes based on their identical peak potentials, but also the high temporal resolution, as fast as $500 \mathrm{~Hz}$, to enable fast measurement to follow rapid biological processes.

Amperometry and differential pulse voltammetry (DPV) are two other commonly used electrochemical techniques for the detection of neurotransmission. Amperometry is a voltammetric technique that a constant potential is applied on the electrode, which is widely used in electrophysiology to study vesicle release events. ${ }^{26}$ Since the signal would be obtained immediately once the analyte diffuses onto the electrode surface, the temporal resolution is completely based on the sampling frequency of the system, which pushes the temporal resolution as fast as one millisecond. ${ }^{26}$ However, unlike FSCV, any compound present that is able to oxidize at that single potential would be oxidized and contributes to the overall signal, giving amperometry very limited selectivity. Biosensors combining a biological component such as enzymes, antibodies, nucleic acids, etc. are used to improve the selectivity, in which a certain analyte is produced to hydrogen peroxide and get detected. But the drawbacks such as high-cost, cumbersome fabrication method, restricted diffusion and slow signal response still exist and limit their further in tissue applications. DPV is another voltammetric technique considered as a derivative of linear sweep voltammetry, with a series of regular voltage pulses superimposed on the potential linear sweep. Using DPV, the effect of the charging current can be decreased by sampling the current just after the decay of the charging potential. Compared to FSCV, the current obtained at DPV is measured immediately before each potential change and the current difference is plotted as a function of potential, providing DPV a greater selectivity and better signal to noise ratio than FSCV. However, the scan rate $(5 \mathrm{mV} / \mathrm{s})$ contributes to the sensitivity and selectivity but also sacrifice temporal resolution (typically more than $1 \mathrm{~s}$ for each measurement).

Microdialysis with high performance liquid chromatography - mass spectrometry (HPLCMS) and functional magnetic resonance imaging (fMRI) are two techniques recently draw lots of interest for neurotransmission detection in brain. Microdialysis is a sampling technique using a 
$200-400 \mu \mathrm{m}$ in diameter sampling probe with a semi-permeable membrane which only allows small biomolecules to pass. Microdialysis is usually coupled with HPLC-MS as separation and detection instruments to provide both high selectivity and sensitivity. However, compared to FSCV, microdialysis has low temporal resolution due to the diffusion as a limitation step in the sampling process. $\mathrm{fMRI}$ is an advanced instrument based upon the nuclear magnetic resonance (NMR) has the advantage of non-invasive detection when used for neurotransmission monitoring. The relatively low special resolution used to be one of the limitation of $f M R I$, but there are a few recent studies with spatial resolution of several millimeters. ${ }^{27,28}$ The limitation, however, is the several second time scale temporal resolution limiting its real - time measurement ability. ${ }^{29}$

In comparison with microdialysis and DPV, FSCV provides faster temporal resolution, but is slower than amperometry. Meanwhile, microdialysis coupled with HPLC/MS and DPV have better selectivity than FSCV, since FSCV cannot distinguish analytes with similar redox reaction potentials, such as dopamine and norepinephrine. Although the pharmacological verification helps verify the chemical identity, ${ }^{30}$ the drug injection process brings more experimental complication and the signal caused by drug itself would be hard to be distinguished from the overall signal. The application of carbon nanomaterials based microelectrodes could help improving the selectivity and distinguishing neurotransmitters which were not distinguishable using CFMEs. ${ }^{5,18}$ Moreover, the incorporation of carbon nanomaterial microelectrodes with FSCV further enhances the sensitivity, spatial and temporal resolution for neurotransmitters detection, which will be introduced in detail in following sections.

\subsection{Carbon Nanomaterial Based Microelectrodes for Neurotransmitter Detection}

\subsubsection{Overview}

Carbon based electrodes are commonly used for neurotransmitter detection because of their low cost, good electron transfer kinetics, and biocompatibility. The high electrocatalytic 
activity of neurotransmitters on carbon surface improves the detection sensitivity. ${ }^{31}$ Moreover, the oxidation/reduction range at carbon electrodes in water environments is wider than other electrode materials, such as platinum ${ }^{4}$ due to a high overvoltage for water oxidation. Ralph Adams and his colleagues realized the utility of electrochemical methods for the study of easily oxidizable neurotransmitters. ${ }^{32}$ They first used a carbon paste electrode implanted in the brain of an anesthetized rat, demonstrating carbon based electrodes could be applied successfully to biological tissue for neurotransmitters detection. ${ }^{32}$ In the late 1970 s, Ponchon et al. introduced $\mathrm{CFMEs}^{33}$, which are still routinely used for in vivo neurotransmitter detection today.

Carbon nanomaterials (CNs) have been a hot research topic for the past two decades, since the discovery of carbon nanotubes (CNTs), and CNs have been incorporated into electrochemical sensors for neurotransmitter detection. The feature size of the nanomaterials is $1-100 \mathrm{~nm}$ and CN-modified electrodes are advantageous because of their large surface-tovolume ratio and large specific surface area. In addition, CNs have enhanced interfacial adsorption properties, better electrocatalytic activity, and fast electron transfer kinetics compared to many traditional electrode materials. In 1996, the first electrochemical application of CNTs was carried out by Britto et al. to detect dopamine at multi-walled carbon nanotube (MWCNT) paste electrodes. ${ }^{34}$ There are many strategies for incorporating CNs into electrochemical sensors for in vivo neurotransmitter measurements. Dip coating and drop casting methods are widely used because of minimal costs and the method is suitable for preparing electrodes with mixtures of CNs, conductive polymers, and/or metal nanoparticles (NPs). Direct growth of CNs and CNTs fibers draws more attention for bio-sensing applications because of the homogenous CNs surface and the preferential exposure of the ends of the CNs which have defect sites that can be functionalized with oxygen to provide abundant electroactive sites for neurotransmitters. ${ }^{31}$ The easy batch fabrication and high reproducibility of the direct growth of CNs and CNT-fiber microelectrodes makes those methods ideal fabrication approaches for single microelectrodes. 
Carbon based nanoelectrodes arrays that contain multiple plates or shanks allow detection of neurotransmitters at network levels. This chapter covers the pros and cons of different fabrication methods and electrode designs.

Several challenges still exist for neurotransmitter monitoring with CNs. First, while many studies characterize the materials, few studies investigate the correlation between the electrochemical performance and the surface properties. Second, fouling on electrode surface in tissue is often a serious problem encountered in the electrochemical analysis. While most of the CN-based sensors reported are not tested in tissue, new studies focusing on bio-fouling and strategies to combat it show that CNs may be advantageous for in vivo testing.

\subsubsection{Carbon Nanomaterial based Electrodes Using Dip Coating/Drop Casting Method}

Dip coating/drop casting fabrication methods are popular for early stage new CN applications due to their low cost and the synergistic effects of combining CNs with other composites such as conductive polymers and/or metal NPs. Polymers can modify the physical and chemical properties of composites and facilitate $\mathrm{CN}$ deposition on the support surface. ${ }^{18}$ The incorporation of CNs with polymers helps to disperse CNTs and graphene in aqueous media via non-covalent interactions, such as van der Waals forces, $\pi^{-} \pi$ interactions, or adsorption/wrapping of polymer. ${ }^{35}$ Better suspensions of $\mathrm{CNs}$ have been reported using heminGO-pristine CNTs complexes on glassy carbon electrode (GCE) ${ }^{36}$, polyhistidine dispersed MWCNT-modified GCE ${ }^{37}$, and acid yellow 9 stabilized MWCNTs on both ITO and GCE ${ }^{38}$. The lowest dopamine LOD was found at the MWCNT/Polyhistidine/GCE (15 nM) ${ }^{37}$. Several conducting polymers have been reported to modify CNTs and graphene based electrodes such as hexadecyl trimethyl ammonium bromide $(\mathrm{CTAB})^{39}$, polypyrrole $(\mathrm{PPy})^{40}$, poly $(3,4-$ ethylenedioxythiophene) (PEDOT) ${ }^{41}$, ${ }^{42}$, poly(vinylferrocene) (PVF) ${ }^{43}$, tryptophan ${ }^{44}, \beta$-cyclodextrin $(\beta-C D)^{45}$, poly (3-methylthiophene) $(\mathrm{PMT})^{46}$, polyaniline (PANI) ${ }^{47-49}$, Polyamide 6/Poly(allylamine 
hydrochloride) (PA6/PAH) ${ }^{50}$, and chitosan ${ }^{51}$. The best DA sensitivity was found on the chitosan grafted graphite on GCE, with an LOD of $4.5 \mathrm{nM}$ because of the high conductivity and biocompatibility of chitosan. ${ }^{51}$ The introduction of polymers with CNs facilitates the dispersion of CNTs and graphene in aqueous media, but polymers have disadvantages as well, such as restricting diffusion, slow temporal resolution, and cumbersome fabrication that is not always reproducible. Moreover, no work directly compares the performance of different polymers.

Metal NPs associated with CNs have been applied to fields such as energy storage, catalysis, and chemical sensors. ${ }^{52}$ Metal NPs perform several functions including increasing the surface area, facilitating heterogeneous electron transfer, enhancing electrical contact between analyte and the $\mathrm{CN}$ surface, inhibiting aggregation of graphene nanosheets, and acting as a spacer. ${ }^{53,54} \mathrm{CN}$-based electrodes incorporated with PtNPs ${ }^{55,56}$, AuNPs ${ }^{57,58}$, CuNPs ${ }^{59}$, and $\mathrm{PbNPs}^{60}$ have been reported. Tsierkezos et al. recently compared different NPs for the first time, examining the detection of dopamine, ascorbic acid, and uric acid on films of nitrogen doped MWCNTs (N-MWCNTs) decorated with RhNPs, PdNPs, IrNPs, PtNPs, and AuNPs ${ }^{61}$ The LODs for dopamine detection improved with the metal NPs and the best LOD was on N-MWCNT/AuNP film $(0.3 \mu \mathrm{M})$. This comparison work would be a good reference for future bio-applications based on metal NPs incorporated with CNs. The main limitations of metal NPs application are biocompatibility issues and the difficulty of construction.

Several works use dip coating/drop casting method to test novel CNs such as nitrogen doped carbon nanofibers, ${ }^{62}$ ordered mesoporous carbon, ${ }^{63}$ and nanodiamond-derived carbon nano-onions ${ }^{64}$. Ko et al. applied thermally oxygenated cup-stacked carbon nanofibers (oxCSCNFs) modified GCE for dopamine detection. ${ }^{65}$ Ox-CSCNFs provide highly ordered graphene edges and oxygen-containing functional groups, which promote fast electron transfer kinetics. Acid oxidized CSCNF-based screen printed electrodes (SPE) increased the sensitivity for dopamine detection because of the mesoporous structure that increased the electroactive surface 
area. $^{66}$ Another important feature of oxygenated CSCNFs is their easy dispersion in water. However, the fabrication and modification process are difficult.

Carbon nanohorns (CNHs) are horn-shaped aggregates of graphene sheets which were first synthesized using laser ablation of pure graphite by ljima. ${ }^{67} \mathrm{CNHs}$ assemble dahlia-like aggregates with an average diameter around $80 \mathrm{~nm}$ and the high purity of SWCNHs (metal-free) offers a unique opportunity for sensing and in tissue application. ${ }^{68,69}$ Valentini et al. used oxidized SWCNH-based SPE for the selective detection of epinephrine in the presence of serotonin and dopamine with LOD of $0.1 \mu \mathrm{M} .^{70}$ The same group reported better electrochemical performance for pristine SWCNHs compared to oxidized SWCNHs for dopamine detection, due to the improved electrical conductivity, electron transfer features, and surface charge of SWCNHs at different degree of oxygen-containing functional groups. ${ }^{71}$ However, the sensitivity (LODs of $0.1 \mu \mathrm{M}$ and $0.4 \mu \mathrm{M}$ at pristine and oxidized SWCNHs, respectively,) needs to be improved. ${ }^{18}$

Du et al. reported a graphene flowers-modified CFMEs to simultaneously detect dopamine, ascorbic acid and uric acid. ${ }^{72}$ Graphene flowers were prepared via electrochemical reduction of graphite oxide sprayed on electrode and these graphene flowers homogeneously decorated on the surface of the electrode. In comparison to unmodified CFMEs, graphene flower-modified CFMEs exhibited high electrocatalytic activity towards the oxidation of dopamine, ascorbic acid, and uric acid. In terms of the possible future in vivo applications, the graphene-flower modified CFMEs is advantageous due to its micrometer-scaled structure (7 $\mu \mathrm{m}$ in diameter), which would minimize tissue damage caused during insertion.

While most CN coating studies coat a GCE for convenience, this is not practical for in vivo studies. Zhang's group used a different strategy to coat acupuncture needles with CNs for the detection of neurotransmitters. ${ }^{73,74}$ Acupuncture needles are a unique microelectrode platform and are widely used in traditional Chinese medicine. Recently, Zhang's group reported a CNT/PEDOT modified acupuncture needle for in vivo detection of serotonin, with LODs of $50 \mathrm{nM}$ and $78 \mathrm{nM}$ in solution and cell medium, respectively. ${ }^{74}$ Although the acupucture needles is a 
promising platform, a systematic study is required to corelate the application of different CNs with their electrochemical performance.

The non-homogenous coating can result in agglomerations, low reproducibility, and slow electron transfer. ${ }^{31,75}$ Screen printing is easy for mass production, but the size and geometry limit their application for deep brain measurement. Most of the dip coated/drop casted CN-based electrodes are characterized by DPV, which has limited application for real-time in vivo measurement due to its slow temporal resolution. However, the low cost of drop casting and dip coating makes it ideal method for early stage electrochemistry study of CNs for neurotransmitter detection.

\subsubsection{Direct Growth of Carbon Nanomaterials on Electrode Substrates}

Direct growth of $\mathrm{CNs}$ on electrode substrates is gaining popularity due to the reproducible mass fabrication and the ability to control the geometry and orientation of the CNs. The ends of CNTs have more defect sites that can be functionalized with oxygen containing groups, which are more likely to be the most electrochemically active sites. $5,18,31,76$ The functional groups, such as carbonyls, phenols, lactones, and carboxylic acids, selectively adsorb cationic dopamine and repel the anionic ascorbate and uric acid at physiological $\mathrm{pH}^{31}$ Direct growth of $\mathrm{CNs}$ is beneficial due to its aligned CNs and high density of edge plane sites, instead of randomly distributed CN films by dip coating/drop casting methods. ${ }^{31,75}$

Vertically-aligned CNTs grown on a microelectrode substrate have high sensitivity for detecting neurotransmitters due to the high density of edge plane sites. ${ }^{77}$ One strategy is to chemically self-assemble vertically-aligned CNTs (VACNTs) on substrates with a solution deposition method. ${ }^{78}$ An alternative strategy is to directly grow CNTs in an aligned manner through chemical vapor deposition (CVD). The Mao group grew VACNTs on carbon fiber supports formed via pyrolysis of iron phthalocyanine as microelectrodes for the detection of ascorbate, DOPAC 
(3,4-Dihydroxyphenylacetic acid), dopamine, and oxygen in rat brain. ${ }^{79,80}$ Use of the VACNTCFMEs avoids manual electrode modification and allows easy fabrication of highly selective, reproducible, and stable microelectrodes. However, the intrinsically low overall conductivity and bad electrochemical stability of the carbon fibers substrates limit their further improvement of electrochemical performance. Moreover, neurotransmitters such as dopamine is detectable at carbon fibers, so the fundamental study at CNTs grown on carbon fiber is vulnerable. Our group reported CNTs directly grown on metal wires fabricated by CVD for sensitive and selective dopamine detection in vivo, and overcame the shortages of carbon fibers by the application of metal substrates. ${ }^{24}$ This was the first study to directly grow CNTs on small diameter metal wires, instead of a planar metal surfaces such as foils, which will be discussed in details in Chapter 2.

Carbon nanospikes (CNS) are a novel CN with tapered and spike-like features less than $50 \mathrm{~nm}$ in diameter. ${ }^{81}$ Our group used plasma-enhanced CVD (PECVD) to directly grow a thin layer of CNS on cylindrical metal substrates under a low-temperature growth condition. ${ }^{25}$ This is the first time that CNS have been grown on metals or on cylindrical substrates. In comparison with the CNT growth, direct growth of CNS does not need catalyst or insulation layer for catalyst deposition, resulting in a direct electrical contact between the CNS and substrate. CNS-Ta microelectrodes exhibited the fastest electron transfer kinetics and lowest LOD (8 \pm 2 nM). CNSmodified electrodes are a promising biosensing material with a similar sensitivity to CNTs but easier growth at low-temperatures and without catalyst or buffer layers.

Diamond-like carbon (DLC) is a metastable form of amorphous carbon that contains a mixture of tetrahedral and trigonal carbon hybridizations fabricated using PECVD. ${ }^{82}$ Silva et al. reported the successful growth of DLC thin film on VACNTs (Ti substrate) and its application for dopamine and epinephrine detection, with LODs of $3.9 \mu \mathrm{M}$ and $4.5 \mu \mathrm{M}$, respectively. ${ }^{83}$ The VACNT scaffolds create conductive pathways that transport current rapidly to all parts of DLC film. However, the dimensions are on the $\mathrm{mm}$ scale and the LODs are not yet sensitive enough for monitoring neurotransmitters in vivo. Similarly, Sainio et al. successfully directly grew MWCNTs 
on the top of DLC thin film electrodes (Si substrate). ${ }^{84}$ The porous structure of MWCNTs/DLC provides fast electron transfer, reduced double layer capacitance, a wide stable water window ($1.5 \mathrm{~V}-2 \mathrm{~V}$ ), and high dopamine sensitivity (LOD of $1.26 \pm 0.23 \mathrm{nM})$. The same group then directly grew carbon nanofibers (CNFs) on DLC thin film (Si substrate), which also exhibited wide water window. ${ }^{85}$ Notably, the CNFs/DLC electrodes could detect glutamate without using any enzymes. However, the detection is not direct but depends on the $\mathrm{pH}$ shift caused by different amounts of glutamate added into solution. Since the local $\mathrm{pH}$ fluctuates $^{86}$, this strategy would not be feasible for glutamate detection in vivo.

Compared to dip coating/drop casting methods, direct growth of CNs has the advantages of homogenous coating, large amount of electroactive sites, and high reproducibility. The best dopamine detection sensitivity was obtained at the MWCNTs/DLC thin film electrodes (1.26 \pm $0.23 \mathrm{nM})^{84}$. However, this electrode is not feasible for in vivo measurement since the dimension of the Si wafer substrate would cause serious tissue damage. In comparison, the application of substrates with several to tens of micron-meter diameter in several studies for CNs growth improves the overall conductivity and minimizes tissue damage.

\subsubsection{Carbon Nanotube-Fiber Microelectrodes}

CNT fibers are a macrostructured carbon material spun from a forest of CNTs. ${ }^{87,88}$ In comparison to randomly structured or tangled CNTs often produced by dip coating or growing, well-aligned CNTs fibers have the advantages of a high electroactivity, fast response, high chemical stability, controllable size, and high resistance to dopamine fouling. ${ }^{89}$ In particular, fabricating CNT fibers directly into electrodes in a manner similar to CFMEs dramatically simplifies the electrode fabrication process and improves the reproducibility. ${ }^{90}$ The first electrochemical application of a CNT fiber sensor was reported in $2003{ }^{91}$ and CNT fiber microelectrodes have been developed for dopamine detection using DPV ${ }^{92}$, chronoamperometry ${ }^{89}$, and FSCV. ${ }^{93,94}$ Two 
distinct routes have been developed for manufacturing CNT fibers: wet-spinning a suspension of CNTs into a bath or direct spinning a fiber from a CNT forest. In solution based wet spinning, CNTs are either dissolved or dispersed in a fluid, extruded out of a spinneret, and coagulated into a solid fiber by extracting the dispersant. ${ }^{95,96}$ Neat CNT fibers can be spun into a polymer bath, such as poly(vinyl alcohol) (PVA $)^{97}$ or poly(ethylene)imine $(\mathrm{PEI})^{98}$, in which the polymer molecules act as coagulators for CNTs to enhance the intertube interactions. Our group has successfully applied PEI/CNT fiber microelectrodes for in vivo monitoring of dopamine and serotonin in brain slice. ${ }^{90}$ Thin, uniform fibers were fabricated by wet spinning CNTs into fibers using PEI as a coagulating polymer. In comparison to PVA/CNT fibers, PEI/CNT fibers have better LODs of dopamine $(4.7 \pm 0.2 \mathrm{nM})$ and lower overpotential.

The alternative fiber production route employs a solid state process where CNTs are either directly spun as a fiber from the synthesis reaction zone ${ }^{99}$ or from a CNT forest grown on a solid substrate. ${ }^{100}$ Continuous CNT yarns, generated by drawing one end of the VACNT arrays, are favorable because of the improved conductivity and CNT alignment. ${ }^{101,102}$ Moreover, compared to CNT fibers which are produced with the incorporation of polymers, CNT yarns are made of CNTs only, without impurities. Schmidt et al. first used CNT yarn microelectrodes for neurotransmitter monitoring. ${ }^{94}$ In this study, the yarns were customizable and tailored for sensitive detection of neurotransmitters. Dopamine concentration fluctuations were successfully detected in acute brain slices using FSCV. Our group utilized a commercially-available CNT yarn fabricated microelectrodes for dopamine detection with LOD of $13 \pm 2 \mathrm{nM} .{ }^{93}$ We also found the dopamine signal is independent to the repetition rate with FSCV at CNT yarn microelectrodes, an advantage over conventionally used CFMEs which lose sensitivity with increasing repetition rate (Fig. 1.2). The high sensitivity with rapid measurement at CNT yarn provides high temporal resolution for neurotransmitters detection and the ability to monitor reaction intermediates and perform neurochemical redox reaction kinetic studies. Moreover, to further improve the sensitivity of CNT 
yarn microelectrodes, our group introduced laser treatment as a simple, reproducible, and efficient surface modification method, which is introduced in detail in Chapter 3.
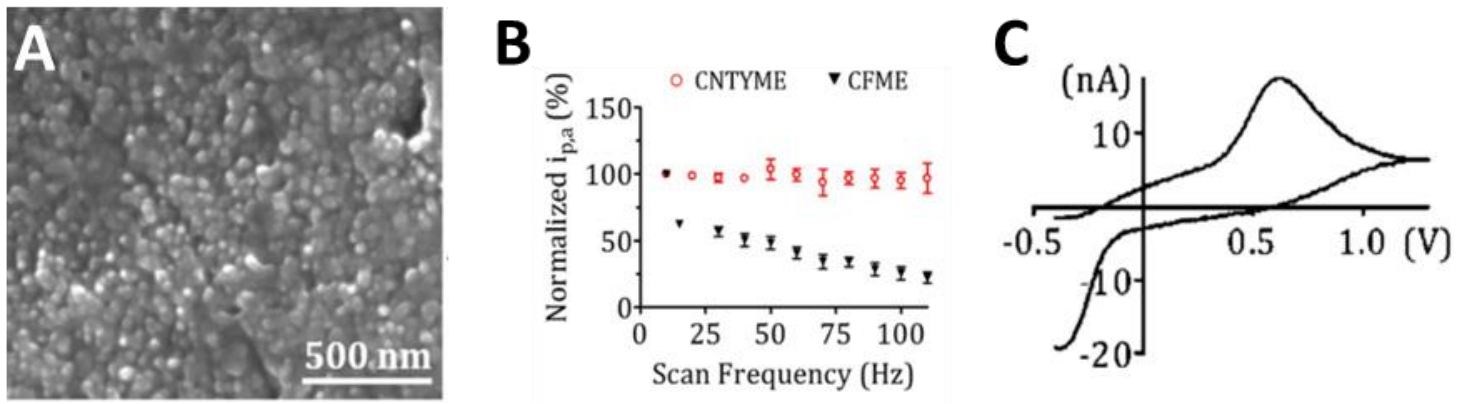

Figure 1.2: CNT yarn microelectrodes. (A) SEM image of CNT yarn microelectrode surface, shows the ends of individual 30-50 nm diameter CNTs bundled tightly together to form a nanostructured surface. (B) Peak oxidation current at CNTYMEs (red circles, $n=10$ ) and CFMEs (black triangles, $n=4$ ). Peak currents were normalized to the current at $10 \mathrm{~Hz}$, and error bars represent the standard error of the mean. (C) High-frequency measurements of $1 \mu \mathrm{M}$ dopamine at CNTYMEs, measured at $2000 \mathrm{~V} / \mathrm{s}$ and $500 \mathrm{~Hz}$. Reproduced with permission from reference ${ }^{93}$, Copyright 2014 American Chemical Society.

The use of similar methods for manufacturing CNT fiber microelectrodes as carbon-fiber microelectrodes dramatically improves the reproducibility and manufacturing efficiency. CNT fibers/yarns with well aligned CNTs have high electroactivity, fast response, high chemical stability, and high fouling resistance. Several kinds of CNT fibers/yarns and surface modifications methods have been applied for in vivo measurement of neurotransmitters, which prove they are a viable alternative to CFMEs.

\subsubsection{Carbon Nanoelectrodes and Carbon Nanomaterial Based Electrode Array}

Nanoscale carbon electrodes are useful for localized detection of neurotransmitters at the level of single cells, single vesicles, as well as single synapses. Small animal models such as Drosophila and zebrafish have attracted more attention recently due to their homologous neurotransmitters with mammals and easy, fast genetic manipulations. ${ }^{26,103,104}$ The small 
dimensions of the central nervous systems of Drosophila and zebrafish requires better spatial resolution and a small, dagger-like electrode than can penetrate through the tough glial sheath barrier with minimal tissue damage. Nanoelectrodes can be used as single sensors or as an array of densely packed nanometer scaled sensors.
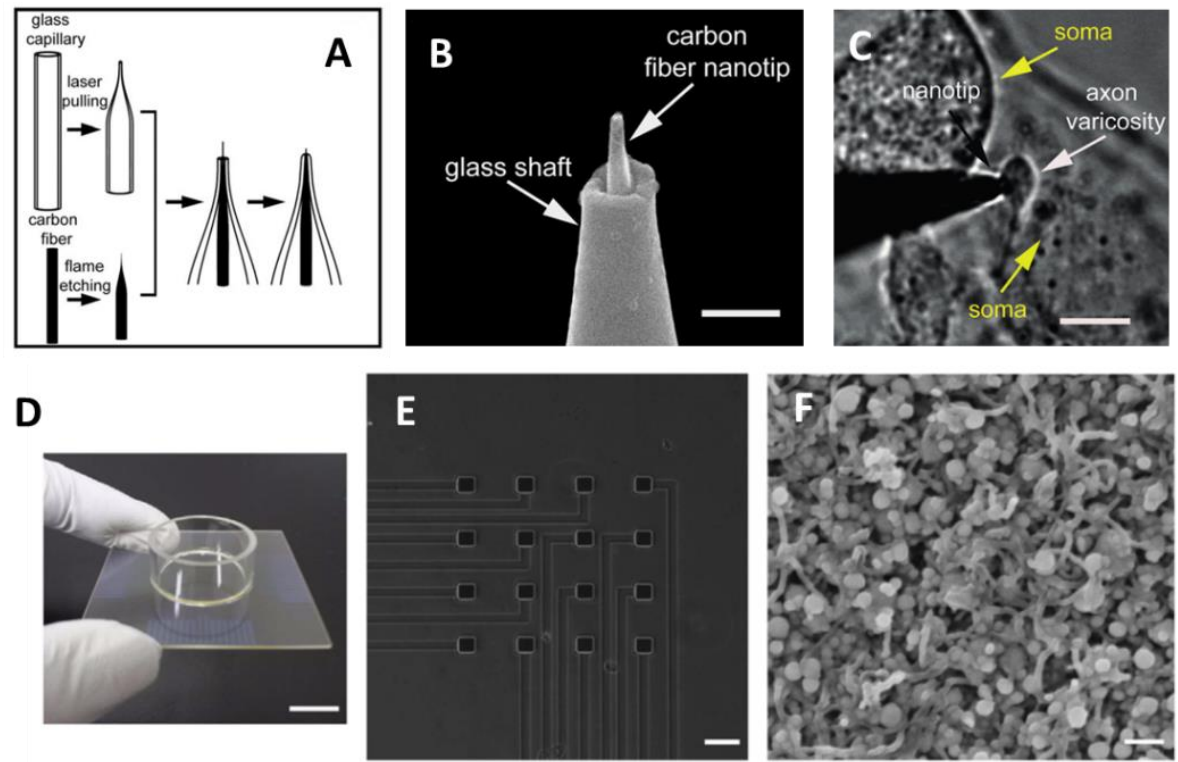

Figure 1.3: Nanoelectrode designs for neurotransmitter detection. (A-C) Carbon fiber nanotip electrodes. (A) Schematic diagram showing main process for fabrication of conical CFNEs. (B) Amplified picture of its tip; the scale bar is $1 \mathrm{~mm}$. (C) Bright-field photomicrographs showing the tip of a sensor inside a synapse between a varicosity of a SCG neuron and the soma of another SCG neuron, scale bars: $5 \mathrm{~mm}$. (D-F) Carbon nanotube-microelectrode arrays. (D) A sample CNT-MEA chip containing 64 electrical recording sites. Scale bar: $1 \mathrm{~cm}$. (E) Phase-contrast image of a CNT-MEA chip showing the spatial configuration of electrodes. Each electrode is $50 \mu \mathrm{m} \times 50$ $\mu \mathrm{m}$. Scale bar, $100 \mu \mathrm{m}$. (F) SEM image of the CNT-MEA chip surface. Scale bar, $200 \mathrm{~nm}$. (A-C) Reproduced with permission from reference ${ }^{105}$, Copyright 2014 Wiley. (D-F) Reprinted with permission from reference ${ }^{106}$, Copyright 2013 Elsevier.

Since the geometric surface area of nanoelectrodes is much smaller than microelectrodes, the application of CNs helps improve the sensitivity with a limited surface area. Li et al. used carbon fiber nanometric electrodes for direct monitoring of exocytotic flux from inside an individual synapse with high resolution in real time. ${ }^{105}$ The facile and robust nanoelectrodes were fabricated by flame-etching with glass-pulled nanopipettes microfabrication, which were subsequently 
etched with a microforge to yield a fine conical tip with diameter less than $100 \mathrm{~nm}$ and controlled shaft length (500 - $2000 \mathrm{~nm}$, Fig. 1.3A-B). The real-time monitoring inside neuronal junctions and neuromuscular junctions using amperometry (Fig. 1.3C) demonstrated the versatility and importance of this new tool.

Our group reported a small, robust, carbon nanopipette electrode (CNPE) for dopamine detection in Drosophila brain. ${ }^{103}$ The CNPEs are fabricated by selectively depositing a carbon layer on the inside of a pulled-quartz capillary that is chemically etched to expose the carbon tip. The electrodes had an average diameter of $250 \mathrm{~nm}$ and controllable exposed length of 5 to 175 $\mu \mathrm{m}$. The LOD of $25 \pm 5 \mathrm{nM}$ with FSCV is similar to CFMEs but the size is 1 order of magnitude smaller in diameter than typical CFMEs. CNPEs were used to detect endogenous dopamine release in Drosophila larvae using optogenetic stimulations, which verified the utility of CNPEs for in vivo neuroscience studies. This is the first work to combine carbon nanoelectrodes and FSCV together, providing a promising solution for the measurements with both high spatial, temporal resolution and high selectivity.

Neurotransmitter release varies not only within discrete substructures of the brain but also across individual cells. ${ }^{107}$ Multi-electrode arrays (MEAs) consolidate multiple sensing elements onto a single device for spatially resolved profiling of neurochemical dynamics as well as simultaneous detection of different analytes. ${ }^{26}$ Suzuki et al. used planar CNTs-MEA chips for the measurements of dopamine release and electrophysiological responses. ${ }^{106}$ These CNT-based MEA chips were fabricated by electroplating bamboo structured MWCNTs on a photolithographyproduced indium tin oxide (ITO) electrode (Fig. 1.3D-F). The LOD for dopamine was $1 \mathrm{nM}$ using amperometry, and the CNT-MEA chips were used to monitor synaptic dopamine release from mouse striatal brain slices. Similarly, Clark et al. reported a functionalized CNT electrode array for dopamine and ascorbic acid detection. ${ }^{108}$ These CNT-MEA chips have 64 individually addressable electrodes of oxygen-functionalized CNTs grown on Pt electrodes. The reduced 
impedance led to improved signal-to-noise ratio. Diner et al. applied a nanocrystalline boron doped diamond (BDD) nanoelectrodes array for dopamine detection, combining the advantages of high spatial resolution nanoelectrode arrays with the promising electrochemical properties of BDD. ${ }^{109}$ The dopamine detection sensitivity was $57.9 \mathrm{nA} \cdot \mu \mathrm{M}^{-1} \mathrm{~cm}^{-2}$ and the selectivity for dopamine over ascorbic acid was high due to the negative charge after oxygen-termination.

Carbon based nanoelectrodes provide robust and sharp tips for neurotransmitter detection at the levels of single synapses, vesicles, and single cells in vivo. Nanoelectrodes provide high spatial resolution, low background current from double layer charging, and minimal tissue damage. The majority of carbon-based nanoelectrodes consist of protruding carbon materials (e.g. carbon fiber) with the exposed length of several to tens of micrometer; therefore the resolution is not nanometer sized in all dimensions. ${ }^{110}$ Compared to single nanoelectrode systems, carbon based nanoelectrodes arrays allow detection of neurotransmitters at network levels. However, the stiff and large silicon wafer and bulk metal substrates limit their in vivo applications. VanDersarl et al. fabricated a flexible implantable neural probe for electrochemical dopamine sensing. ${ }^{111}$ The electrode arrays were fabricated on silicon carrier wafers and then released from the substrate by chemical/electrochemical dissolution, which makes the electrodes array flexible and used for long-term neurotransmitter monitoring in vivo. As the development of $\mathrm{CNs}$ and new electrodes continues, we expect more attention will be focused on increasing the sensitivity and nanometerscaled resolution in vivo, especially for measurements at single synapses and smaller model organisms.

\subsubsection{D-Printing Technologies for Carbon Nanomaterial based Microsensors Fabrication}

3D-printing, defined as a process used to fabricate three dimensional objects based on the digitally controlled deposition, has the potential to transform science and technology by creating bespoke, low-cost applications that previously required dedicated facilities to make. ${ }^{112,113}$ 
This would not only place traditionally expensive chemical engineering technology within reach of typical laboratories, ${ }^{114}$ but also could revolutionize access to healthcare and the chemical sciences in general in the developing world. ${ }^{115} 3 \mathrm{D}$ printing approaches are recently increasingly useful as a rapid prototyping fabrication tool in several scientific and medical applications, such as tissue-growth scaffolds, ${ }^{116,117}$ microvascular systems, ${ }^{118}$ orthopedic implants, ${ }^{119}$ rehabilitation aid tools, ${ }^{120}$ electronic device, ${ }^{121}$ and chemical reactors. ${ }^{122}$ Electrochemistry is another branch of science that can benefit from 3D-printing technologies, paving the way for both of design and fabrication of cheaper, higher performing, and bespoke electrochemical devices. ${ }^{112}$ With the rapid development of 3D printing, increasing needs are required to incorporate 3D printing to carbon nanomaterial based microsensors fabrication. In particular, 3D-printing has been employed not only to customize electrode design such as voltammetric cells or microfluidic systems, for the application of existing materials, but also to produce conductive electrodes with special shapes or compositions.

Motivated by the idea of a future whereby users can download a design for a product from the internet, modify it to suit an intended purpose and then rapidly produce any number of such items at low cost on a portable, robust devices, ${ }^{123} 3 \mathrm{D}$ printed electrochemistry reactionware has been widely applied. Symes et al. reported the usage of 3D printed reactionware for organic and inorganic synthesis, which include printed-in catalysts and other architectures, enabling in situ electrochemical monitoring of reactions with digital feedback mechanism. ${ }^{122}$ The reactionware provides a cheap and automated electrochemical analysis platform, successfully transferring techniques to typical synthetic laboratories. Microfluidic devices and flow cells for electrochemical analysis can also be fabricated using 3D printing. Snowden et al. designed a versatile flow cell which easily accommodate different types of electrodes for specific electrochemical experiments. ${ }^{124}$ The flow cell is assembled simply by resting the micro-fabricated component on the electrode of interest and securing with 3D printed thread. More recently, Erkal et al. reported 
a 3D printed microfluidic devices with integrated versatile and reusable electrode. ${ }^{125}$ With the electrode housed in commercially available fittings, various electrode materials are easily added to a threaded receiving port printed on the device, enabling a module-like approach to the experimental design. A dopamine LOD of $500 \mathrm{nM}$ at an embedded glassy carbon electrodes on the 3D printed microfluidic system was achieved. Notably, the electrode fitting is removable and reusable, instead of the traditional one-time-use evaporation/deposition-based electrodes. Recently, we developed a novel microelectrode batch fabrication method based on a 3D-printed mold and using bio-compatible, easy and quick curing polyimide resin as insulating agent. ${ }^{126}$ The 3D-printed mold is low cost, customizable to change the electrode shape, and allows 40 electrodes to be made simultaneously, which will be discussed in Chapter 6 . We also directly printed out electrodes platform specifically for CNT yarn and fibers for the sensitive detection of neurotransmitters. Moreover, the 3D printed electrodes are fully compatible to conventionally used screen printed electrodes, so no additional device or accessories is required other than a SPE based electrochemistry workstation setup, which dramatically reduce the cost and simply the fabrication process. The electrochemical characterization and electrode design/fabrication will be introduced in detail in Chapter 7.

A trend for the electrochemical application using 3D printing is the direct fabrication of electrode using conductive material, which could be used directly as electrode material for electrochemical study, or as conductive electrode substrates followed by further surface modification. Extrusion-based 3D printing (e.g. robocasting or direct ink writing) is the most versatile, which has a broad material selection including ceramics, metal alloys, polymers, and can directly fabricate electrodes. ${ }^{127,128}$ Since graphene oxide has noteworthy printing capability and unique viscoelastic properties as an aqueous dispersion, 3D printing of graphene oxide has been successfully performed in to different structural forms, such as cathode and anode for lithium-ion batteries. ${ }^{129}$ Although 3D printed graphene oxide electrode has not been applied for 
bio-sensing applications yet, the good electrical conductivity, abundant electroactive sites, as well as the bespoke design using $3 \mathrm{D}$ printing technique, will attract more neurochemists to explore the potential application for neurotransmission monitoring. Recently, 3D printed metal electrodes as custom-shaped platform has been used as electrochemical devices due to the rapid development of metal 3D printing technology. ${ }^{130}$ Ambrosi et al. used 3D printed stainless steel electrodes as platform for $\mathrm{pH}$ sensing, functionalized by the deposition of $\mathrm{IrO}_{2}$ film, which allows the fabrication of specific electrode designs that can be tailored to facilitate specific functions and properties. ${ }^{112}$ In the near future, we expect to observe more 3D printed electrochemical sensors for bio-sensing application.

\subsubsection{Conclusions}

Dip coating/drop casting method is an ideal method for testing early stage electrochemical performance study of novel CNs or combining CNs with polymers and/or metal NPs for neurotransmitter detection. However, disadvantages include the non-homogenous coating, low reproducibility, and complicated fabrication as well as restricted diffusion and decreased conductivity which jeopardize electrochemical performance. ${ }^{5}$ In comparison, direct growth of CNs on microelectrodes leads to high conductivity, larger amount of electroactive sites, higher reproducibility, and easier batch fabrication. CNTs fiber/yarn microelectrodes are expected to be a potential alternative to CFMEs for in vivo neurotransmitter detection because the highly aligned CNTs lead to controllable electrochemical properties. Moreover, the electrochemical properties of CNT yarn microelectrodes can be controlled by surface treatments, which allows researchers to tune the material depending on the requirements of sensitivity and selectivity to different analytes. ${ }^{23}$ The development of flexible electrodes is another future path since most electrodes/arrays are made from stiff silicon or metal substrates, which limit their in vivo application. In addition, with the rapid development of 3D printing, increasing needs are required 
to incorporate $3 \mathrm{D}$ printing to carbon nanomaterial based microsensors fabrication. Overall, the best LODs reported is achieved by the planar CNTs-MEA chips with chronoamperometric measurements of dopamine release $(1 \mathrm{nM}) .{ }^{106}$ And the best LOD for microelectrodes is $4.7 \pm 0.2$ $\mathrm{nM}$, achieved by PEI/CNT fiber microelectrodes. ${ }^{90}$ While most of the current experiments have concentrated on dopamine detection, future studies could expand the analytes detected and strive for better sensitivity and selectivity at smaller electrodes for a range of neurochemicals.

\subsection{Challenges and Future Directions}

Although CN-based electrochemical sensors for neurotransmitter detection have been extensively studied for the past two decades, several challenges still exist for their routine in vivo application. First, more information is needed about the correlation between surface properties and the sensor electrochemical performance. Studies that correlate surface and electrochemical properties will lead towards rational sensor design and better prediction of the likely electrochemical properties of new materials. Second, fouling of electrode surface in tissue is often a serious problem which negatively affects the analytical performance including sensitivity, detection limit, and measurement reproducibility for the in vivo implanted electrodes. While many studies do not test new electrodes in tissue, there are researchers who are beginning to study biofouling and how CN-based strategies could improve fouling. In this section, studies overcoming these challenges and provide a perspective of the future direction for in vivo electrochemical sensors for direct neurotransmitter detection will be discussed.

\subsubsection{Correlation between Electrochemical Performance and Carbon Nanomaterial Surface} Properties

Dopamine is a catecholamine with heterogeneous electron transfer that is strongly dependent on surface properties and electrocatalysis. ${ }^{31}$ The electrostatic interactions between 
negatively charged oxide groups on the carbon surface and positively charged dopamine at physiological $\mathrm{pH}$ as well as the $\pi-\pi$ stacking between $\mathrm{CNs}$ and the catechol significantly influence the redox reactions. ${ }^{131}$ The redox processes of dopamine depend on the existence of surface oxygen-containing functional groups, such as carbonyl or carboxylate, which can readily adsorb neurotransmitters and facilitate electron transfer. ${ }^{18,31}$ While CNs can enhance the surface for dopamine redox reactions, correlating the exact properties of the nanomaterials with electrochemistry is challenging.

CNTs and graphene have abundant $\mathrm{sp}^{3}$-hybridized, edge plane carbons that can be oxidized to provide functional groups compared to conventionally used glassy carbon and carbon fiber. ${ }^{132}$ The morphology of $\mathrm{CNs}$ and the density of edge plane sites significantly influence the redox reactions. Muguruma et al. reported an electrochemical study of the synthetic pathway of dopamine (DA), dopamine-o-quinone (DAQ), leucodopaminechrome (LDAC), and dopaminechrome (DAC) at an electrode made of long-length (hundred microns) CNTs dispersed in solution with surfactant cellulose. ${ }^{133}$ The electrically conducting networks of long-length CNTs had long m-electron networks and were denser than those of normal-length CNT electrode resulting in reduced activation potential for oxidation and efficient propagation of the electron produced by $L D A C \rightleftharpoons D A C$ reaction. The observation of predominant second redox couple (LDAC $\rightleftharpoons \mathrm{DAC})$ at long-length CNTs could be used as a quantitative and selective detection of dopamine just as in the conventional strategy of direct oxidation of dopamine (DA $\rightleftharpoons \mathrm{DOQ})$. These two works show that CNT alignment, length, and density affect both sensitivity and the dopamine redox reaction products. Since metal substrates that are not sensitive to dopamine enable the study of the interaction of dopamine with CNTs without the convolution of possible substrate reactivity, CNTs grown on metal substrate would provide a promising platform for the fundamental study of the interface interactions. 
Surface properties of $\mathrm{CN}$-based electrodes also influence their temporal resolution with FSCV measurements. FSCV is the preferred in vivo electrochemical method because the CV fingerprint aids in analyte identification and the typical $100 \mathrm{~ms}$ temporal resolution allows neurotransmitter measurements on a subsecond time scale.4,134-136 At traditional CFMEs, dopamine signal decreases with increasing scan repetition frequency and the $10 \mathrm{~Hz}$ repetition frequency is a compromise between temporal resolution and sensitivity. ${ }^{22,137}$ While methods to improve temporal resolution by raising the scan rate have been explored, the oxidation current still decreases with increasing scan repetition frequency. ${ }^{138,139}$ Our group has recently discovered that at CNTYMEs, the dopamine current does not change significantly with increasing scan repetition frequency: at a scan rate of $2000 \mathrm{~V} / \mathrm{s}$, dopamine can be detected, without any loss in sensitivity, with scan frequencies up to $500 \mathrm{~Hz} \cdot{ }^{93}$ Thus, the temporal resolution is four times faster without a decrease in signal. The cause of this increased temporal resolution is differences in dopamine and DOQ adsorption and desorption kinetics; at CNTYMEs, the rates of desorption for dopamine and DOQ are almost identical, while at CFMEs, the rate of desorption for DOQ is over an order of magnitude higher than that for dopamine. Therefore, CNT morphology and roughness affect the temporal resolution of measurements and CNTYMEs enable high speed measurements with high sensitivity, since there is no compromise in current with higher sampling rates. Surface roughness is another important factor affecting the dopamine redox reaction. In our recent work, ${ }^{23}$ laser-etched CNT yarn microelectrodes were used for the in vivo detection of dopamine, which will be discussed in detail in Chapter 3 .

A better understanding of the correlation between surface properties CNs, including alignment, length, edge plane sites, surface functionalization, and surface roughness, and the corresponding electrochemical performance of neurotransmitters will allow researchers to tailor the electrochemistry by applying different CNs or surface modifications. The better aligned CNTs on electrode surface provide higher conductivity and more electroactive sites for dopamine 
compared to non-oriented, spaghetti-like CNTs. Long-length CNTs form denser conducting networks than the normal-length CNTs, facilitating detection of the second dopamine redox couple, which would be used as a quantitative and selective detection of dopamine. Surface roughness on the order of dopamine diffusion length momentarily traps dopamine and allows higher temporal resolution measurements than at conventional CFMEs. A better fundamental understanding of how $\mathrm{CN}$ properties lead to electrochemical response will enable rational development of future electrodes. Recently, we correlated the surface and electrochemical properties for neurochemical detection at 3 types of materials: CNT fibers produced by wet spinning with (1) polyethylenimine (PEI/CNT) or (2) chlorosulfonic acid (CA/CNT), and (3) CNT yarns made by solid-based CNT drawing. Overall, small crevices, high conductivity, and abundant oxygen groups lead to high sensitivity for amine neurotransmitters, such as dopamine and serotonin. These results show that surface properties of CNT fibers can be used to predict their electrochemical performance, which will be introduced in Chapter 5.

\subsubsection{Effect of Bio-Fouling on Carbon Nanomaterial based Microelectrodes for in vivo} Neurotransmitter Monitoring

Long-term monitoring the real-time dynamics of neurotransmitters in vivo is essential to understand the role of chemical communication in cognitive function with diverse dynamics through learning and developmental processes. ${ }^{140-142}$ Approaches such as non-invasive brain imaging can provide long-term monitoring of brain activity, but its low spatial resolution precludes cellular level investigation. ${ }^{143}$ Optical imaging techniques can achieve neuron-resolution mapping but are limited in terms of penetration depth. ${ }^{144}$ Electrochemical microsensors incorporated with carbon nanomaterials such as CNT yarn can provide high spatial resolution for dynamics studies in deep brain regions. ${ }^{94}$ However, the effect of bio-fouling happened on carbon nanomaterial based microelectrodes for in vivo implantation is still elusive. 
Surface fouling can severely affect the sensitivity and reproducibility of electrochemical sensing in vivo and CNs have been proposed as a technique to alleviate surface fouling. Electrode fouling involves the surface passivation by a fouling agent that forms an increasingly impermeable layer on the electrode, which inhibits the direct contact of the analyte with the electrode surface for electron transfer. ${ }^{145}$ Two main types of fouling arise: (1) passive biological fouling by tissue species such as proteins and lipids and (2) active fouling by byproducts of the analyte redox reaction that form the insulating layer. The fouling agent can adhere to the electrode surface as a result of hydrophobic, hydrophilic, or electrostatic interactions. ${ }^{31,146}$ For example, the products of serotonin oxidation are very reactive and may form an insulating film on the electrode surface, thus jeopardizing the long-term stability of the electrodes. ${ }^{147,148}$ Commonly used antifouling strategies include the addition of a protective layer or barrier on the CNs substrate (polymeric film ${ }^{149,150}$ or metal nanoparticles ${ }^{151-153}$ ), electrode surface modifications ${ }^{154,155}$, and electrochemical activation ${ }^{156,157}$. This section examines recent efforts to study fouling resistance study at CN electrodes and develop $\mathrm{CN}$-based antifouling strategies.

The hydrophilic surface of CNs electrodes facilitates their antifouling properties. ${ }^{145}$ Electrodes with hydrophobic surfaces promote adsorption of hydrophobic molecules, including aromatic compounds and proteins. ${ }^{145}$ These hydrophobic interactions are sufficiently favorable, especially in an aqueous condition, that they are typically irreversible..$^{31}$ In contrast, fouling caused by hydrophilic interactions tends to be more reversible. ${ }^{158}$ Therefore, many strategies aimed at reducing fouling by the application of CNs target reducing the hydrophobicity of the electrode surface. Xiang et al. reported VACNTs grown on carbon-fiber electrodes exhibited good resistance against electrode fouling in high concentration $(0.5 \mathrm{mM})$ ascorbate solution, with only $2 \%$ signal decrease for 30 min continuous amperometric measurements. ${ }^{79}$ Our group found the PEI/CNT fiber microelectrodes have promising antifouling properties for serotonin. Prevention of electrode fouling from the metabolite of serotonin, 5-hydroxyindoleacetic acid, is particularly 
important because its physiological concentration is 10 times higher than that of serotonin. ${ }^{90} \mathrm{At}$ the PEI/CNT fiber microelectrode, the peak oxidation current for serotonin remained constant upon incubation in 5-hydroxyindolacetic acid for $2 \mathrm{~h}$ and serotonin was successfully measured in brain slices. Similarly, Harreither et al. reported pristine PVA/CNT fiber microelectrodes exhibited a high resistance to dopamine fouling. ${ }^{89}$ While the electrode lost half of its initial signal in 15 min at high concentration dopamine solution $(1 \mathrm{mM})$, there was no signal change in $100 \mu \mathrm{M}$ dopamine for $2 \mathrm{~h}$ as the insulating patches grew slower on the CNT fiber surfaces than on CFMEs. Since the basal levels of extracellular dopamine are around 10-30 $\mathrm{nM}$ and phasic release during fast burst of firing resulting in release from 0.1 to $1 \mu \mathrm{M}^{5}$, lack of fouling at low concentrations could make them advantageous in vivo. The antifouling properties of both the VACNT-carbon fiber, PVA/CNT and PEI/CNT fiber electrodes are likely due to the presence of large amount of functionalized edge plane sites on CNTs which makes the surface hydrophilic.

Boron doped diamond exhibits a high chemical stability and high resistance to surface fouling, usually at the expense of decreased reaction kinetics in comparison to other CNs. ${ }^{159,160}$ Patel et al. compared several different carbon electrodes for their antifouling properties for dopamine, including glassy carbon, oxygen-terminated polycrystalline boron-doped diamond (pBDD), edge plane pyrolytic graphite (EPPG), basal plane pyrolytic graphite (BPPG), and the basal surface of highly oriented pyrolytic graphite (HOPG) ${ }^{161}$ Although pBDD was found to be the least susceptible to surface fouling even at relatively high dopamine concentration, the reaction kinetics were relatively slow. In contrast, the reaction of $100 \mu \mathrm{M}$ dopamine at pristine basal plane HOPG had fast kinetics and only minor susceptibility toward surface fouling.

Fouling due to adsorption of biological macromolecules, such as proteins, is also important to minimize for optimal sensor performance because adsorption affects mass transport and electron transfer kinetics. ${ }^{162}$ Soluble proteins are often hydrophilic on the surface to interact with the aqueous environment and hydrophobic on the inside to maintain protein folding or the binding 
of hydrophobic materials. ${ }^{145}$ Thus, proteins can foul electrode through both hydrophilic and hydrophobic interactions. Harreither et al. reported PVA/CNT fiber electrodes were more resistant to fouling than the traditional carbon-fiber electrodes for dopamine detection in the presence of bovine serum albumin (BSA), which accounts for about $50 \%$ of total plasma protein content. ${ }^{163}$ The presence of albumin reduces the impact of dopamine fouling because the sulfur on the BSA competed with the amine moiety for the nucleophilic binding to the oxidized catechol (a critical step initiating dopamine fouling) and therefore reduced the rate of dopamine polymerization on the electrode surface. Similarly, Liu et al. recently reported a new, effective solution for electrode calibration for in vivo measurements by pretreating MWCNTS/CFMEs with BSA. ${ }^{164}$ The ratio of the sensitivity obtained with the post-calibration to that obtained with pre-calibration containing BSA was about $94 \%$. Essentially, the strategy is to pre-foul the electrodes before implantation, and thus they will not be sensitive to BSA overadsorption or changes in their surface hydrophobicity upon implantation (Fig. 1.4). The electrodes also exhibited a high selectivity for ascorbate against dopamine, DOPAC, uric acid, and serotonin. Chen et al. evaluated the impact of the surface charges and morphology of PEDOT modified gold electrodes on the detection of dopamine, ascorbic acid, and uric acid in the presence of proteins, including BSA, lysozyme, and fibrinogen. ${ }^{165}$ The adsorption of positively charged lysozyme promoted sensitive detection of ascorbic acid and uric acid, and all protein adsorption lowered the dopamine sensitivity. Although a gold electrode was used in this study, it provides a good reference for the future study focusing on the neurotransmitters detection at $\mathrm{CN}$-based electrodes in the presence of different proteins. 


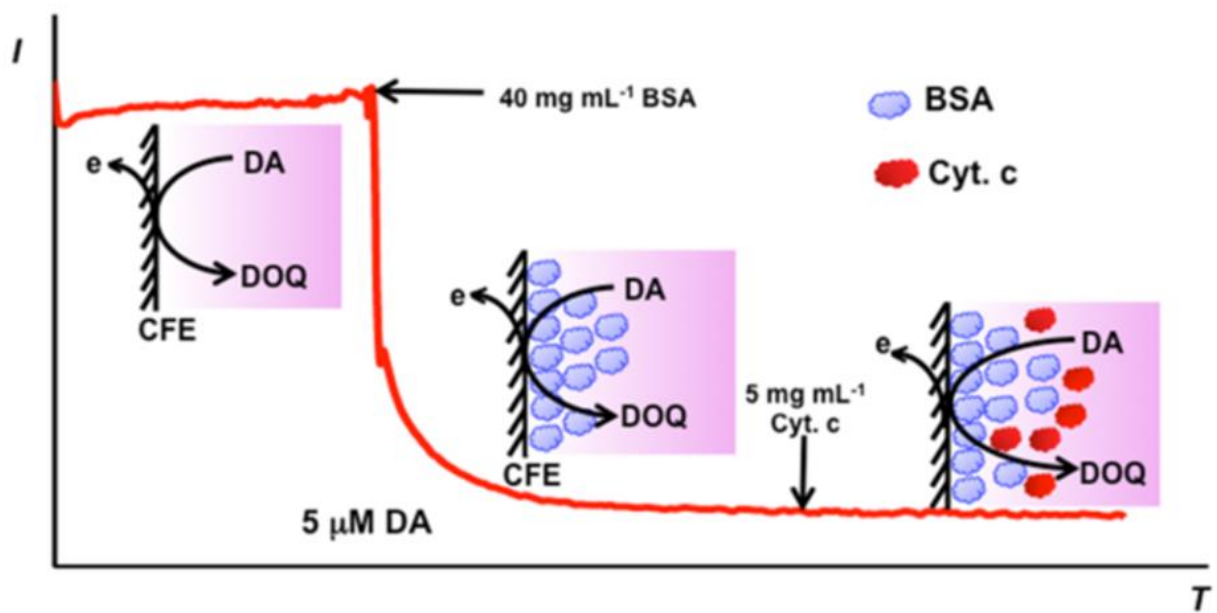

Figure 1.4: Schematic illustration of amperometric response at the electrode/electrolyte interface in different concentrations of protein. Reprinted with permission from reference ${ }^{164}$, Copyright 2016 American Chemical Society.

In summary, CNs are being used to change the surface chemistry of microsensors and improve the surface fouling resistance, giving $\mathrm{CNs}$ based implantable microsensors long-term neurotransmission monitoring ability. ${ }^{166}$ While polymer incorporated CNs electrodes, such as PEDOT:PSS, ${ }^{149}$ PEDOT:GO, ${ }^{167}$ and Nafion-CNT, ${ }^{168}$ have been proposed to provide fouling resistance for in vivo, polymers can affect temporal response and not all are effective. ${ }^{150}$ Thus, the future research will likely focus on the promising fouling resistance at CNs such as BDD, HOPG, VACNTs, and CNT fibers. In particular, hydrophilic CN surfaces tend to have better antifouling properties because of the higher reversibility of hydrophilic interactions with the surface than the hydrophobic interactions. Oxidation of the surface of $\mathrm{CNs}$ to introduce oxygen-containing functional groups is the easiest method to make the surface more hydrophilic, and oxidized CNs are preferred for in vivo neurotransmitters detection because they lead to both fouling resistance and high sensitivity. The surface roughness is another important factor contributing to the fouling resistance, contributing to the observed antifouling properties of superhydrophobic electrodes. ${ }^{169,170}$ Promising methods for future cleaning of adsorbed materials include applying single anodic/cathodic potentials $s^{157,171,172}$ or a train of pulses to periodically clean the electrode 
surface ${ }^{173,174}$. The antifouling mechanism for anodic/cathodic potential relies on altering the amount of oxygen functionalities, which affects surface hydrophilicity. ${ }^{145,146}$ Future studies should focus on how surface properties affect fouling, evaluations of long term anti-fouling in actual tissue. In chapter 4 of this thesis, the anti-fouling property at implantable CNT yarn microelectrodes with different surface modifications will be evaluated. 


\subsection{References}

(1) Matthews, G. NEUROTRANSMITTER RELEASE. Annu. Rev. 1996, 19, 219-233.

(2) Koob, G. F. Frameworks of Alcohol Addiction: Alcohol Addiction as a Reward Deficit and Stress Surfeit Disorder. Neurobiol. Alcohol Depend. 2014, 4 (August), 3-28.

(3) Janezic, S.; Threlfell, S.; Dodson, P. D.; Dowie, M. J.; Taylor, T. N.; Potgieter, D.; Parkkinen, L.; Senior, S. L.; Anwar, S.; Ryan, B.; Deltheil, T.; Kosillo, P.; Cioroch, M.; Wagner, K.; Ansorge, O.; Bannerman, D. M.; Bolam, J. P.; Magill, P. J.; Cragg, S. J.; Wade-Martins, R. Deficits in Dopaminergic Transmission Precede Neuron Loss and Dysfunction in a New Parkinson Model. Proc. Natl. Acad. Sci. U. S. A. 2013, 110 (42), E4016-25.

(4) Robinson, D. L.; Venton, B. J.; Heien, M. L. a V; Wightman, R. M. Detecting Subsecond Dopamine Release with Fast-Scan Cyclic Voltammetry in Vivo. Clin. Chem. 2003, 49 (10), 1763-1773.

(5) Jacobs, C. B.; Peairs, M. J.; Venton, B. J. Review: Carbon Nanotube Based Electrochemical Sensors for Biomolecules. Anal. Chim. Acta 2010, 662 (2), 105-127.

(6) Millar, J.; Stamford, J. A.; Kruk, Z. L.; Wightman, R. M. Department of Physiology and * Pharmacology, The London Hospital Medical College, Turner Street, London E1 2AD, U.K.; and * * Department of Chemistry, Indiana University, Bloomington, IN 47405, U.S.A. 1985, 109, 341-348.

(7) Jankowski, J. A.; Leszczyszyn, D. J.; Diliberto, E. J. Temporally Resolved Catecholamine Spikes Correspond to Single Vesicle Release from Individual Chromaffin Cells. 1991, 88 (December), 10754-10758.

(8) Mirzaei, M.; Sawan, M. Microelectronics-Based Biosensors Dedicated to the Detection of Neurotransmitters: A Review. 2014, 17981-18008.

(9) Gan, T.; Hu, S. Electrochemical Sensors Based on Graphene Materials. Microchim. Acta 2011, 175 (1-2), 1-19.

(10) Liu, J.; Liu, Z.; Barrow, C. J.; Yang, W. Molecularly Engineered Graphene Surfaces for Sensing Applications: A Review. Anal. Chim. Acta 2014, 859 (2015), 1-19.

(11) Lawal, A. T. Synthesis and Utilisation of Graphene for Fabrication of Electrochemical Sensors. Talanta 2014, 131 (2015), 424-443.

(12) Zhan, B.; Li, C.; Yang, J.; Jenkins, G.; Huang, W.; Dong, X. Graphene Field-Effect Transistor and Its Application for Electronic Sensing. Small 2014, 4042-4065.

(13) Chen, A.; Chatterjee, S. Nanomaterials Based Electrochemical Sensors for Biomedical Applications. Chem. Soc. Rev. 2013, 42, 5425-5438.

(14) Zhang, L.; Wang, J.; Tian, Y. Electrochemical in-Vivo Sensors Using Nanomaterials Made from Carbon Species, Noble Metals , or Semiconductors. No. 2014, 1471-1484.

(15) Balasubramanian, K.; Kern, K. 25th Anniversary Article: Label-Free Electrical Biodetection Using Carbon Nanostructures. Adv. Mater. 2014, 26 (8), 1154-1175.

(16) Gao, C.; Guo, Z.; Liu, J.-H.; Huang, X.-J. The New Age of Carbon Nanotubes: An Updated Review of Functionalized Carbon Nanotubes in Electrochemical Sensors. Nanoscale 2012, $4,1948$. 
(17) Vera, S. Chemical Sensors Based on Polymer Composites with Carbon Nanotubes and Graphene: The Role of the Polymer. J. Mater. Chem. A 2014, 14289-14328.

(18) Yang, C.; Denno, M. E.; Pyakurel, P.; Venton, B. J. Recent Trends in Carbon NanomaterialBased Electrochemical Sensors for Biomolecules: A Review. Anal. Chim. Acta 2015, 887, 17-37.

(19) Robinson, D. L.; Hermans, A.; Seipel, A. T.; Wightman, R. M. Monitoring Rapid Chemical Communication in the Brain. 2008, 2554-2584.

(20) Garris, P. A.; Neurobiology, I.; Hall, V. REGULATION OF TRANSIENT DOPAMINE CONCENTRATION GRADIENTS TN THE MICROENVIRONMENT SURROUNDING NERVE TERMINALS IN THE RAT STRIATUM. 1992, 51 (I), 55-64.

(21) Robinson, D. L.; Venton, B. J.; Heien, M. L. a V; Wightman, R. M. Detecting Subsecond Dopamine Release with Fast-Scan Cyclic Voltammetry in Vivo. Clin. Chem. 2003, 49 (10), 1763-1773.

(22) Venton, B. J.; Troyer, K. P.; Wightman, R. M. Response Times of Carbon Fiber Microelectrodes to Dynamic Changes in Catecholamine Concentration. Anal. Chem. 2002, 74 (3), 539-546.

(23) Yang, C.; Trikantzopoulos, E.; Nguyen, M. D.; Jacobs, C. B.; Wang, Y.; Mahjouri-Samani, M.; Ivanov, I. N.; Venton, B. J. Laser Treated Carbon Nanotube Yarn Microelectrodes for Rapid and Sensitive Detection of Dopamine in Vivo. ACS Sensors 2016, 1 (5), 508-515.

(24) Yang, C.; Jacobs, C. B.; Nguyen, M. D.; Ganesana, M.; Zestos, A. G.; Ivanov, I. N.; Puretzky, A. A.; Rouleau, C. M.; Geohegan, D. B.; Venton, B. J. Carbon Nanotubes Grown on Metal Microelectrodes for the Detection of Dopamine. Anal. Chem. 2016, 88 (1), 645652 .

(25) Zestos, A. G.; Yang, C.; Jacobs, C. B.; Hensley, D.; Venton, B. J. Carbon Nanospikes Grown on Metal Wires as Microelectrode Sensors for Dopamine. Analyst 2015, 140 (21), 7283-7292.

(26) Bucher, E. S.; Wightman, R. M. Electrochemical Analysis of Neurotransmitters. Annu. Rev. Anal. Chem. (Palo Alto. Calif). 2015, 8, 239-261.

(27) Goense, J.; Bohraus, Y.; Logothetis, N. K. fMRI at High Spatial Resolution: Implications for BOLD-Models. 2016, 10 (June), 1-13.

(28) Ferraro, S.; Mandelli, M. L.; Bertolino, N.; Ghielmetti, F.; Epifani, F.; Nigri, A.; Bruzzone, M. G.; Donato, S. Di; Savoiardo, M.; Mariotti, C. Frontal Cortex BOLD Signal Changes in Premanifest Huntington Disease A Possible fMRI Biomarker. 2014.

(29) Sahib, A. K.; Mathiak, K.; Erb, M.; Elshahabi, A.; Klamer, S.; Scheffler, K.; Focke, N. K.; Ethofer, T. Effect of Temporal Resolution and Serial Autocorrelations in Event-Related Functional MRI. 2016, 0 (August 2015), 1-9.

(30) Kuhr, W. G.; Wightman, R. M.; Rebec, G. V. Dopaminergic Neurons: Simultaneous Measurements of Dopamine Release and Single-Unit Activity during Stimulation of the Medial Forebrain Bundle. 1987, 418, 122-128.

(31) McCreery, R. L. Advanced Carbon Electrode Materials for Molecular Electrochemistry. Chem. Rev. 2008, 108 (7), 2646-2687. 
(32) Kissinger, P.; Hart, J.; Adams, R. N. Voltammetry in Brain Tissue - a New Neurophysilogical Measurement. Brain Res. 1973, 55, 209-213.

(33) Ponchon, J. L.; Cespuglio, R.; Gonon, F.; Jouvet, M.; Pujol, J. F. Normal Pulse Polarography with Carbon Fiber Electrodes for in Vitro and in Vivo Determination of Catecholamines. Anal. Chem. 1979, 51 (9), 1483-1486.

(34) Britto, P. J.; Santhanam, K. S. V; Ajayan, P. M. Carbon Nanotube Electrode for Oxidation of Dopamine. Bioelectrochemistry Bioenerg. 1996, 41 (1), 121-125.

(35) Mittal, V. Surface Modification of Nanotube Fillers; WILEY-VCH Verlag: Weinheim, Germany, 2011.

(36) Zhang, Y.; Xia, Z.; Liu, H.; Yang, M.; Lin, L.; Li, Q. Hemin-Graphene Oxide-Pristine Carbon Nanotubes Complexes with Intrinsic Peroxidase-like Activity for the Detection of $\mathrm{H} 2 \mathrm{O} 2$ and Simultaneous Determination for Trp, AA, DA, and UA. Sensors Actuators B Chem. 2013, 188 (2013), 496-501.

(37) Dalmasso, P. R.; Pedano, M. L.; Rivas, G. a. Dispersion of Multi-Wall Carbon Nanotubes in Polyhistidine: Characterization and Analytical Applications. Anal. Chim. Acta 2012, 710 (2012), 58-64.

(38) Kumar, S. A.; Wang, S.-F.; Yang, T. C.-K.; Yeh, C.-T. Acid Yellow 9 as a Dispersing Agent for Carbon Nanotubes: Preparation of Redox Polymer-Carbon Nanotube Composite Film and Its Sensing Application towards Ascorbic Acid and Dopamine. Biosens. Bioelectron. 2010, 25 (12), 2592-2597.

(39) Yang, Y. J.; Li, W. CTAB Functionalized Graphene Oxide/multiwalled Carbon Nanotube Composite Modified Electrode for the Simultaneous Determination of Ascorbic Acid, Dopamine, Uric Acid and Nitrite. Biosens. Bioelectron. 2014, 56, 300-306.

(40) Si, P.; Chen, H.; Kannan, P.; Kim, D.-H. Selective and Sensitive Determination of Dopamine by Composites of Polypyrrole and Graphene Modified Electrodes. Analyst 2011, 136 (24), 5134-5138.

(41) Wang, W.; Xu, G.; Cui, X. T.; Sheng, G.; Luo, X. Enhanced Catalytic and Dopamine Sensing Properties of Electrochemically Reduced Conducting Polymer Nanocomposite Doped with Pure Graphene Oxide. Biosens. Bioelectron. 2014, 58, 153-156.

(42) Xu, G.; Li, B.; Cui, X. T.; Ling, L.; Luo, X. Electrodeposited Conducting Polymer PEDOT Doped with Pure Carbon Nanotubes for the Detection of Dopamine in the Presence of Ascorbic Acid. Sensors Actuators B Chem. 2013, 188 (2013), 405-410.

(43) Şen, M.; Tamer, U.; Pekmez, N. Ö. Carbon Nanotubes/alizarin Red S-poly(vinylferrocene) Modified Glassy Carbon Electrode for Selective Determination of Dopamine in the Presence of Ascorbic Acid. J. Solid State Electrochem. 2011, 16 (2), 457-463.

(44) Lian, Q.; He, Z.; He, Q.; Luo, A.; Yan, K.; Zhang, D.; Lu, X.; Zhou, X. Simultaneous Determination of Ascorbic Acid, Dopamine and Uric Acid Based on Tryptophan Functionalized Graphene. Anal. Chim. Acta 2014, 823, 32-39.

(45) Tan, L.; Zhou, K.-G.; Zhang, Y.-H.; Wang, H.-X.; Wang, X.-D.; Guo, Y.-F.; Zhang, H.-L. Nanomolar Detection of Dopamine in the Presence of Ascorbic Acid at $\beta$ Cyclodextrin/graphene Nanocomposite Platform. Electrochem. commun. 2010, 12 (4), 557-560. 
(46) Quan, D. P.; Tuyen, D. P.; Lam, T. D.; Tram, P. T. N.; Binh, N. H.; Viet, P. H. Electrochemically Selective Determination of Dopamine in the Presence of Ascorbic and Uric Acids on the Surface of the Modified Nafion/single Wall Carbon nanotube/poly(3Methylthiophene) Glassy Carbon Electrodes. Colloids Surf. B. Biointerfaces 2011, 88 (2), 764-770.

(47) Shieh, Y.-T.; Tu, Y.-Y.; Wang, T.-L.; Lin, R.-H.; Yang, C.-H.; Twu, Y.-K. Apparent Electrocatalytic Activities of Composites of Self-Doped Polyaniline, Chitosan, and Carbon Nanotubes. J. Electroanal. Chem. 2013, 704 (2013), 190-196.

(48) Rodthongkum, N.; Ruecha, N.; Rangkupan, R.; Vachet, R. W.; Chailapakul, O. GrapheneLoaded Nanofiber-Modified Electrodes for the Ultrasensitive Determination of Dopamine. Anal. Chim. Acta 2013, 804, 84-91.

(49) Liu, J.; Luo, J.; Liu, R.; Jiang, J.; Liu, X. Micelle-Encapsulated Multi-Wall Carbon Nanotubes with Photosensitive Copolymer and Its Application in the Detection of Dopamine. Colloid Polym. Sci. 2013, 292 (1), 153-161.

(50) Mercante, L. A.; Pavinatto, A.; Iwaki, L. E. O.; Scagion, V. P.; Zucolotto, V.; Oliveira, O. N.; Mattoso, L. H. C.; Correa, D. S. Electrospun Polyamide 6/poly(allylamine Hydrochloride) Nanofibers Functionalized with Carbon Nanotubes for Electrochemical Detection of Dopamine. ACS Appl. Mater. Interfaces 2015, 7 (8), 4784-4790.

(51) Palanisamy, S.; Sakthinathan, S.; Chen, S.-M.; Thirumalraj, B.; Wu, T.-H.; Lou, B.-S.; Liu, $X$. Preparation of $\beta$-Cyclodextrin Entrapped Graphite Composite for Sensitive Detection of Dopamine. Carbohydr. Polym. 2016, 135, 267-273.

(52) Muszynski, R.; Seger, B.; Kamat, P. V. Decorating Graphene Sheets with Gold Nanoparticles. J. Phys. Chem. C 2008, $112(14)$, 5263-5266.

(53) Jackowska, K.; Krysinski, P. New Trends in the Electrochemical Sensing of Dopamine. Anal. Bioanal. Chem. 2013, 405 (11), 3753-3771.

(54) Ponnusamy, V. K.; Mani, V.; Chen, S.-M.; Huang, W.-T.; Jen, J.-F. Rapid Microwave Assisted Synthesis of Graphene Nanosheets/polyethyleneimine/gold Nanoparticle Composite and Its Application to the Selective Electrochemical Determination of Dopamine. Talanta 2014, 120 (2014), 148-157.

(55) Guo, D.-J.; Qiu, X.-P.; Chen, L.-Q.; Zhu, W.-T. Multi-Walled Carbon Nanotubes Modified by Sulfated TiO2 - A Promising Support for Pt Catalyst in a Direct Ethanol Fuel Cell. Carbon N. Y. 2009, 47 (7), 1680-1685.

(56) Zan, X.; Bai, H.; Wang, C.; Zhao, F.; Duan, H. Graphene Paper Decorated with a 2D Array of Dendritic Platinum Nanoparticles for Ultrasensitive Electrochemical Detection of Dopamine Secreted by Live Cells. Chem. - A Eur. J. 2016, 22 (15), 5204-5210.

(57) Yuan, D.; Chen, S.; Yuan, R.; Zhang, J.; Liu, X. An ECL Sensor for Dopamine Using Reduced Graphene Oxide/multiwall Carbon Nanotubes/gold Nanoparticles. Sensors Actuators B Chem. 2014, 191 (2014), 415-420.

(58) Komathi, S.; Gopalan, A. I.; Lee, K.-P. Nanomolar Detection of Dopamine at Multi-Walled Carbon Nanotube Grafted Silica Network/gold Nanoparticle Functionalised Nanocomposite Electrodes. Analyst 2010, 135 (2), 397-404.

(59) Zheng, S.; Huang, Y.; Cai, J.; Guo, Y. Nano-Copper-MWCNT-Modified Glassy Carbon Electrode for Selective Detection of Dopamine. Int. J. Electrochem. Sci 2013, 8 (2013), 
12296-12307.

(60) Tyszczuk-Rotko, K.; Sadok, I. The New Application of Boron Doped Diamond Electrode Modified with Nafion and Lead Films for Simultaneous Voltammetric Determination of Dopamine and Paracetamol. Electroanalysis 2016, 1-11.

(61) Tsierkezos, N. G.; Othman, S. H.; Ritter, U.; Hafermann, L.; Knauer, A.; Köhler, J. M.; Downing, C.; McCarthy, E. K. Electrochemical Analysis of Ascorbic Acid, Dopamine, and Uric Acid on Nobel Metal Modified Nitrogen-Doped Carbon Nanotubes. Sensors Actuators, B Chem. 2016, 231, 218-229.

(62) Sun, J. Y.; Li, L.; Zhang, X. P.; Liu, D.; Lv, S. M.; Zhu, D. R.; Wu, T.; You, T. A. Simultaneous Determination of Ascorbic Acid, Dopamine and Uric Acid at a Nitrogen-Doped Carbon Nanofiber Modified Electrode. RSC Adv. 2015, 5 (16), 11925-11932.

(63) Martín-Yerga, D.; Rama, E. C.; Costa-García, A. Electrochemical Characterization of Ordered Mesoporous Carbon Screen-Printed Electrodes. J. Electrochem. Soc. 2016, 163 (5), B176-B179.

(64) Yang, J.; Zhang, Y.; Kim, D. Y. Electrochemical Sensing Performance of NanodiamondDerived Carbon Nano-Onions: Comparison with Multiwalled Carbon Nanotubes, Graphite Nanoflakes, and Glassy Carbon. Carbon N. Y. 2016, 98, 74-82.

(65) Ko, S.; Tatsuma, T.; Sakoda, A.; Sakai, Y.; Komori, K. Electrochemical Properties of Oxygenated Cup-Stacked Carbon Nanofiber-Modified Electrodes. Phys. Chem. Chem. Phys. 2014, 16 (24), 12209.

(66) Lamas-Ardisana, P. J.; Fanjul-Bolado, P.; Costa-García, A. Manufacture and Evaluation of Cup-Stacked Carbon Nanofiber-Modified Screen Printed Electrodes as Electrochemical Tools. J. Electroanal. Chem. 2016, 775, 129-134.

(67) lijima, S.; Yudasaka, M.; Yamada, R.; Bandow, S.; Suenaga, K.; Kokai, F.; Takahashi, K. Nano-Aggregates of Single-Walled Graphitic Carbon Nano-Horns. Chem. Phys. Lett. 1999, 309 (3-4), 165-170.

(68) Martín, A.; Hernández-Ferrer, J.; Vázquez, L.; Martínez, M.-T.; Escarpa, A. Controlled Chemistry of Tailored Graphene Nanoribbons for Electrochemistry: A Rational Approach to Optimizing Molecule Detection. RSC Adv. 2014, 4 (1), 132.

(69) Zhu, S.; Li, H.; Niu, W.; Xu, G. Simultaneous Electrochemical Determination of Uric Acid, Dopamine, and Ascorbic Acid at Single-Walled Carbon Nanohorn Modified Glassy Carbon Electrode. Biosens. Bioelectron. 2009, 25 (4), 940-943.

(70) Valentini, F.; Ciambella, E.; Conte, V.; Sabatini, L.; Ditaranto, N.; Cataldo, F.; Palleschi, G.; Bonchio, M.; Giacalone, F.; Syrgiannis, Z.; Prato, M. Highly Selective Detection of Epinephrine at Oxidized Single-Wall Carbon Nanohorns Modified Screen Printed Electrodes (SPEs). Biosens. Bioelectron. 2014, 59, 94-98.

(71) Valentini, F.; Ciambella, E.; Boaretto, A.; Rizzitelli, G.; Carbone, M.; Conte, V.; Cataldo, F.; Russo, V.; Casari, C. S.; Chillura-Martino, D. F.; Caponetti, E.; Bonchio, M.; Giacalone, F.; Syrgiannis, Z.; Prato, M. Sensor Properties of Pristine and Functionalized Carbon Nanohorns. Electroanalysis 2016, 1-12.

(72) Du, J.; Yue, R.; Ren, F.; Yao, Z.; Jiang, F.; Yang, P.; Du, Y. Novel Graphene Flowers Modified Carbon Fibers for Simultaneous Determination of Ascorbic Acid, Dopamine and Uric Acid. Biosens. Bioelectron. 2014, 53, 220-224. 
(73) Tang, L.; Du, D.; Yang, F.; Liang, Z.; Ning, Y.; Wang, H.; Zhang, G.-J. Preparation of Graphene-Modified Acupuncture Needle and Its Application in Detecting Neurotransmitters. Sci. Rep. 2015, 5 (January), 11627.

(74) Li, Y.-T.; Tang, L.-N.; Ning, Y.; Shu, Q.; Liang, F.-X.; Wang, H.; Zhang, G.-J.; Hurtak, J. J.; Lee, H.; Ernst, E.; Nardini, C.; Langevin, H. M.; Churchill, D. L.; Cipolla, M. J.; Sierpina, V. S.; Frenkel, M. A.; Bienenstock, J.; Hsiao, S. H.; Tsai, L. J.; Chang, F. C.; Zhong, A. M.; Wu, J. L.; Hu, Y. L.; Dean, D. M.; Dana, B.; Yoseph, A. M.; Shi, Y.; Zhang, D.; Wu, M. L.; Pönicke, K.; Gergs, U.; Buchwalow, I. B.; Hauptmann, S.; Neumann, J.; Coplan, J. D.; Njagi, J.; Ball, M.; Best, M.; Wallace, K. N.; Andreescu, S.; Du, D.; Xiang, L.; Li, Y. T.; Li, Y. T.; Tang, L.; Treacy, M. M. J.; Ebbesen, T. W.; Gibson, J. M.; Harreither, W.; Vitale, F.; Summerson, S. R.; Aazhang, B.; Kemere, C.; Pasquali, M.; Hu, P.; Tanii, T.; Zhang, G. J.; Hosaka, T.; Ohdomari, I.; Bello, A.; Wang, W.; Xu, G.; Cui, X.; Sheng, G.; Luo, X.; Chen, G. Z.; Bhandari, S.; Deepa, M.; Srivastava, A. K.; Joshi, A. G.; Kant, R.; Xiang, L.; Luo, X.; Lu, F. S.; Jiang, L.; Lin, X.; Li, J.; Lin, X.; Babaei, A.; Babazadeha, M.; Babaei, A.; Taheri, A. R.; Han, H. S.; You, J. M.; Jeong, H.; Jeon, S.; Han, H. S.; Xue, C.; Gil, H.; Bae, K.-H.; Kim, L.; Kim, S. C.; Soh, K.-S.; Wang, X.; Racké, K.; Schwörer, H.; Racké, K.; Reimann, A.; Schwörer, H.; Kilbinger, H.; Xu, G.; Li, B.; Cui, X. T.; Ling, L.; Luo, X.; Liang, F. X.; Zhu, M. F. In Vivo Monitoring of Serotonin by Nanomaterial Functionalized Acupuncture Needle. Sci. Rep. 2016, 6 (February), 28018.

(75) Jacobs, C. B.; Vickrey, T. L.; Venton, B. J. Functional Groups Modulate the Sensitivity and Electron Transfer Kinetics of Neurochemicals at Carbon Nanotube Modified Microelectrodes. Analyst 2011, 136 (17), 3557.

(76) Liu, J. Fullerene Pipes. Science (80-. ). 1998, 280 (5367), 1253-1256.

(77) Álvarez-Martos, I.; Fernández-Gavela, A.; Rodríguez-García, J.; Campos-Alfaraz, N.; García-Delgado, a. B.; Gómez-Plaza, D.; Costa-García, A.; Fernández-Abedul, M. T. Electrochemical Properties of Spaghetti and Forest like Carbon Nanotubes Grown on Glass Substrates. Sensors Actuators B Chem. 2014, 192 (2014), 253-260.

(78) Xiao, N.; Venton, B. J. Rapid, Sensitive Detection of Neurotransmitters at Microelectrodes Modified with Self-Assembled SWCNT Forests. Anal. Chem. 2012, 84 (18), 7816-7822.

(79) Xiang, L.; Yu, P.; Hao, J.; Zhang, M.; Zhu, L.; Dai, L.; Mao, L. Vertically Aligned Carbon Nanotube-Sheathed Carbon Fibers as Pristine Microelectrodes for Selective Monitoring of Ascorbate in Vivo. Anal. Chem. 2014, 86 (8), 3909-3914.

(80) Xiang, L.; Yu, P.; Zhang, M.; Hao, J.; Wang, Y.; Zhu, L.; Dai, L.; Mao, L. Platinized Aligned Carbon Nanotube-Sheathed Carbon Fiber Microelectrodes for in Vivo Amperometric Monitoring of Oxygen. Anal. Chem. 2014, 86 (10), 5017-5023.

(81) Sheridan, L. B.; Hensley, D. K.; Lavrik, N. V.; Smith, S. C.; Schwartz, V.; Liang, C.; Wu, Z.; Meyer, H. M.; Rondinone, a. J. Growth and Electrochemical Characterization of Carbon Nanospike Thin Film Electrodes. J. Electrochem. Soc. 2014, 161 (9), H558-H563.

Robertson, J. Diamond-like Amorphous Carbon. Mater. Sci. Eng. R Reports 2002, 37 (46), 129-281.

Silva, T. A.; Zanin, H.; May, P. W.; Corat, E. J.; Fatibello-Filho, O. Electrochemical Performance of Porous Diamond-like Carbon Electrodes for Sensing Hormones, Neurotransmitters, and Endocrine Disruptors. ACS Appl. Mater. Interfaces 2014, 6 (23), 21086-21092. 
(84) Sainio, S.; Palom??ki, T.; Rhode, S.; Kauppila, M.; Pitk??nen, O.; Selk??!??, T.; Toth, G.; Moram, M.; Kordas, K.; Koskinen, J.; Laurila, T. Carbon Nanotube (CNT) Forest Grown on Diamond-like Carbon (DLC) Thin Films Significantly Improves Electrochemical Sensitivity and Selectivity towards Dopamine. Sensors Actuators, B Chem. 2015, 211, 177-186.

(85) Sainio, S.; Palomäki, T.; Tujunen, N.; Protopopova, V.; Koehne, J.; Kordas, K.; Koskinen, J.; Meyyappan, M.; Laurila, T. Integrated Carbon Nanostructures for Detection of Neurotransmitters. Mol. Neurobiol. 2015, 52 (2), 859-866.

(86) Dengler, A. K.; Wightman, R. M.; McCarty, G. S. Microfabricated Collector-Generator Electrode Sensor for Measuring Absolute $\mathrm{pH}$ and Oxygen Concentrations. Anal. Chem. 2015, 87 (20), 10556-10564.

(87) Vilatela, J. J.; Marcilla, R. Tough Electrodes: Carbon Nanotube Fibers as the Ultimate Current Collectors/Active Material for Energy Management Devices. Chem. Mater. 2015, $27(20), 6901-6917$.

(88) Lu, W.; Zu, M.; Byun, J. H.; Kim, B. S.; Chou, T. W. State of the Art of Carbon Nanotube Fibers: Opportunities and Challenges. Adv. Mater. 2012, 24 (14), 1805-1833.

(89) Harreither, W.; Trouillon, R.; Poulin, P.; Neri, W.; Ewing, A. G.; Safina, G. Carbon Nanotube Fiber Microelectrodes Show a Higher Resistance to Dopamine Fouling. Anal. Chem. 2013, 85 (15), 7447-7453.

(90) Zestos, A. G.; Jacobs, C. B.; Trikantzopoulos, E.; Ross, A. E.; Venton, B. J. Polyethylenimine Carbon Nanotube Fiber Electrodes for Enhanced Detection of Neurotransmitters. Anal. Chem. 2014, 86, 8568-8575.

(91) Wang, J.; Deo, R. P.; Poulin, P.; Mangey, M. Carbon Nanotube Fiber Microelectrodes. J. Am. Chem. Soc. 2003, 125 (48), 14706-14707.

(92) Viry, L.; Derré, A.; Poulin, P.; Kuhn, A. Discrimination of Dopamine and Ascorbic Acid Using Carbon Nanotube Fiber Microelectrodes. Phys. Chem. Chem. Phys. 2010, 12 (34), 99939995.

(93) Jacobs, C. B.; Ivanov, I. N.; Nguyen, M. D.; Zestos, A. G.; Venton, B. J. High Temporal Resolution Measurements of Dopamine with Carbon Nanotube Yarn Microelectrodes. Anal. Chem. 2014, 86 (12), 5721-5727.

(94) Schmidt, A. C.; Wang, X.; Zhu, Y.; Sombers, L. a. Carbon Nanotube Yarn Electrodes for Enhanced Detection of Neurotransmitter Dynamics in Live Brain Tissue. ACS Nano 2013, 7864-7873.

(95) Li, Z.; Liu, Z.; Sun, H.; Gao, C. Superstructured Assembly of Nanocarbons: Fullerenes, Nanotubes, and Graphene. Chem. Rev. 2015, 115 (15), 7046-7117.

(96) Behabtu, N.; Young, C. C.; Tsentalovich, D. E.; Kleinerman, O.; Wang, X.; Ma, A. W. K.; Bengio, E. A.; ter Waarbeek, R. F.; de Jong, J. J.; Hoogerwerf, R. E.; Fairchild, S. B.; Ferguson, J. B.; Maruyama, B.; Kono, J.; Talmon, Y.; Cohen, Y.; Otto, M. J.; Pasquali, M. Strong, Light, Multifunctional Fibers of Carbon Nanotubes with Ultrahigh Conductivity. Science (80-. ). 2013, 339 (6116), 182-186.

(97) Vigolo, B.; Pénicaud, A.; Coulon, C.; Sauder, C.; Pailler, R.; Journet, C.; Bernier, P.; Poulin, P. Macroscopic Fibers and Ribbons of Oriented Carbon Nanotubes. Science (80-. ). 2000, 290 (5495), 1331-1334. 
(98) Muñoz, E.; Suh, D. S.; Collins, S.; Selvidge, M.; Dalton, A. B.; Kim, B. G.; Razal, J. M.; Ussery, G.; Rinzler, A. G.; Martínez, M. T.; Baughman, R. H. Highly Conducting Carbon Nanotube/polyethyleneimine Composite Fibers. Adv. Mater. 2005, 17(8), 1064-1067.

(99) Koziol, K.; Vilatela, J.; Moisala, A.; Motta, M.; Cunniff, P.; Sennett, M.; Windle, A. HighPerformance Carbon Nanotube Fiber. Science (80-. ). 2007, 318 (5858), 1892-1895.

(100) Zhang, M.; Atkinson, K. R.; Baughman, R. H. Multifunctional Carbon Nanotube Yarns by Downsizing an Ancient Technology. Science 2004, 306 (5700), 1358-1361.

(101) Mayhew, E.; Prakash, V. Thermal Conductivity of High Performance Carbon Nanotube Yarn-like Fibers. J. Appl. Phys. 2014, 115 (17), 174306.

(102) Jiang, K.; Li, Q.; Fan, S. Nanotechnology: Spinning Continuous Carbon Nanotube Yarns. Nature 2002, 419 (6909), 801.

(103) Rees, H. R.; Anderson, S. E.; Privman, E.; Bau, H. H.; Venton, B. J. Carbon Nanopipette Electrodes for Dopamine Detection in Drosophila. Anal. Chem. 2015, 87 (7), 3849-3855.

(104) Traver, D.; Paw, B. H.; Poss, K. D.; Penberthy, W. T.; Lin, S.; Zon, L. I. Transplantation and in Vivo Imaging of Multilineage Engraftment in Zebrafish Bloodless Mutants. Nat. Immunol. 2003, 4 (12), 1238-1246.

(105) Li, Y.; Zhang, S.-H.; Wang, L.; Xiao, R.; Liu, W.; Zhang, X.; Zhou, Z.; Amatore, C.; Huang, W. Nanoelectrode for Amperometric Monitoring of Individual Vesicular Exocytosis Inside Single Synapses. Angew. Chemie Int. Ed. 2014, 53 (46), 12456-12460.

(106) Suzuki, I.; Fukuda, M.; Shirakawa, K.; Jiko, H.; Gotoh, M. Carbon Nanotube Multi-Electrode Array Chips for Noninvasive Real-Time Measurement of Dopamine, Action Potentials, and Postsynaptic Potentials. Biosens. Bioelectron. 2013, 49, 270-275.

(107) Wightman, R. M.; Heien, M. L. A. V; Wassum, K. M.; Sombers, L. A.; Aragona, B. J.; Khan, A. S.; Ariansen, J. L.; Cheer, J. F.; Phillips, P. E. M.; Carelli, R. M. Dopamine Release Is Heterogeneous within Microenvironments of the Rat Nucleus Accumbens. Eur. J. Neurosci. 2007, 26 (7), 2046-2054.

(108) Clark, J.; Chen, Y.; Silva, S. R. P. Low Impedance Functionalised Carbon Nanotube Electrode Arrays for Electrochemical Detection. Electroanalysis 2016, 28 (1), 58-62.

(109) Dincer, C.; Ktaich, R.; Laubender, E.; Hees, J. J.; Kieninger, J.; Nebel, C. E.; Heinze, J.; Urban, G. A. Nanocrystalline Boron-Doped Diamond Nanoelectrode Arrays for Ultrasensitive Dopamine Detection. Electrochim. Acta 2015, 185, 101-106.

(110) Colombo, M. L.; Sweedler, J. V.; Shen, M. Nanopipet-Based Liquid-Liquid Interface Probes for the Electrochemical Detection of Acetylcholine, Tryptamine, and Serotonin via Ionic Transfer. Anal. Chem. 2015, 87 (10), 5095-5100.

(111) Van Dersarl, J. J.; Mercanzini, A.; Renaud, P. Integration of 2D and 3D Thin Film Glassy Carbon Electrode Arrays for Electrochemical Dopamine Sensing in Flexible Neuroelectronic Implants. Adv. Funct. Mater. 2015, 25 (1), 78-84.

(112) Ambrosi, A.; Pumera, M. 3D-Printing Technologies for Electrochemical Applications. Chem. Soc. Rev. 2016, 45 (10), 2740-2755.

(113) Pinnell, R. C.; Almajidy, R. K.; Hofmann, U. G. Versatile 3D-Printed Headstage Implant for Group Housing of Rodents. J. Neurosci. Methods 2016, 257, 134-138. 
(114) Yager, P.; Edwards, T.; Fu, E.; Helton, K.; Nelson, K.; Tam, M. R.; Weigl, B. H. Microfluidic Diagnostic Technologies for Global Public Health. 2006, 442 (July).

(115) Cook, T. R.; Dogutan, D. K.; Reece, S. Y.; Surendranath, Y.; Teets, T. S.; Nocera, D. G. Solar Energy Supply and Storage for the Legacy and Nonlegacy Worlds. Chem. Rev. 2010, 6474-6502.

(116) Lee, K.; Wang, S.; Dadsetan, M.; Yaszemski, M. J.; Lu, L. Enhanced Cell Ingrowth and Proliferation through Three-Dimensional Nanocomposite Scaffolds with Controlled Pore Structures. Biomacromolecules 2010, 11, 682-689.

(117) Mannoor, M. S.; Jiang, Z.; James, T.; Kong, Y. L.; Malatesta, K. A.; Soboyejo, W. O.; Verma, N.; Gracias, D. H.; Mcalpine, M. C. 3D Printed Bionic Ears. Nano Lett. 2013, 13, 2634-2639.

(118) Therriault, D.; White, S. R.; Lewis, J. A. Chaotic Mixing in Three-Dimensional Microvascular Networks Fabricated by Direct-Write Assembly. 2003, 2 (April), 265-272.

(119) Mackinnon, J.; Daurka, J. Use of 3D Printing in Orthopaedic Surgery. 2014, 2963 (May), 2963.

(120) Hsu, L. H.; Huang, G. F.; Lu, C. T.; Hong, D. Y.; Liu, S. H. The Development of a Rapid Prototyping Prosthetic Socket Coated with a Resin Layer for Transtibial Amputees. 2010, 34 (March), 37-45.

(121) Zhao, C.; Wang, C.; lii, R. G.; Beirne, S.; Shu, K.; Wallace, G. G. Electrochemistry Communications Three Dimensional ( $3 D$ ) Printed Electrodes for Interdigitated Supercapacitors. Electrochem. commun. 2014, 41, 20-23.

(122) Symes, M. D.; Kitson, P. J.; Yan, J.; Richmond, C. J.; Cooper, G. J. T.; Bowman, R. W.; Vilbrandt, T.; Cronin, L. Integrated 3D-Printed Reactionware for Chemical Synthesis and Analysis Mark. Nat. Chem. 2012, 4 (5), 349-354.

(123) Geissler, B. M.; Xia, Y. Patterning: Principles and Some New Developments. 2004, No. 15, 1249-1269.

(124) Snowden, M. E.; King, P. H.; Covington, J. A.; Macpherson, J. V; Unwin, P. R. Fabrication of Versatile Channel Flow Cells for Quantitative Electroanalysis Using Prototyping. 2010, 82 (8), 3124-3131.

(125) Walton, E. L.; Mcnamara, S.; Scott, R.; Spence, D. M. Lab on a Chip Versatile and Reusable Electrodes. Lab Chip 2014, 2023-2032.

(126) Trikantzopoulos, E.; Yang, C.; Ganesana, M.; Wang, Y.; Venton, B. J. Novel Carbon-Fiber Microelectrode Batch Fabrication Using a 3D-Printed Mold and Polyimide Resin. Analyst 2016, 3-7.

(127) Ober, T. J.; Foresti, D.; Lewis, J. A. Active Mixing of Complex Fluids at the Microscale. Proc. Natl. Acad. Sci. 2015, 112 (40), 2-7.

(128) Ellis, J.; Mavromatos, N. E.; Nanopoulos, D. V.; Sakharov, a. S. Cosmology: Synchrotron Radiation and Quantum Gravity. Nature 2004, 428 (6981), 2481.

(129) Fu, K.; Wang, Y.; Yan, C.; Yao, Y.; Chen, Y.; Dai, J.; Lacey, S.; Wang, Y.; Wan, J.; Li, T.; Wang, Z.; Xu, Y.; Hu, L. Graphene Oxide-Based Electrode Inks for 3D-Printed Lithium-Ion Batteries. Adv. Mater. 2016, 28 (13), 2587-2594.

(130) Hirt, L.; Ihle, S.; Pan, Z.; Dorwling-Carter, L.; Reiser, A.; Wheeler, J. M.; Spolenak, R.; 
Vörös, J.; Zambelli, T. Template-Free 3D Microprinting of Metals Using a Force-Controlled Nanopipette for Layer-by-Layer Electrodeposition. Adv. Mater. 2016, 28 (12), 2311-2315.

(131) Cao, M.; Fu, A.; Wang, Z.; Liu, J.; Kong, N.; Zong, X.; Liu, H.; Gooding, J. J. Electrochemical and Theoretical Study of $\Pi-\Pi$ Stacking Interactions between Graphitic Surfaces and Pyrene Derivatives. J. Phys. Chem. C 2014, 118 (5), 2650-2659.

(132) Yang, X.; Feng, B.; He, X.; Li, F.; Ding, Y.; Fei, J. Carbon Nanomaterial Based Electrochemical Sensors for Biogenic Amines. Microchim. Acta 2013, 180 (11-12), 935956.

(133) Muguruma, H.; Inoue, Y.; Inoue, H.; Ohsawa, T. Electrochemical Study of Dopamine at Electrode Fabricated by Cellulose-Assisted Aqueous Dispersion of Long-Length Carbon Nanotube. J. Phys. Chem. C 2016, 120 (22), 12284-12292.

(134) Robinson, D. L.; Hermans, A.; Seipel, A. T.; Wightman, R. M. Monitoring Rapid Chemical Communication in the Brain. Chem. Rev. 2008, 108 (7), 2554-2584.

(135) Nguyen, M. D.; Lee, S. T.; Ross, A. E.; Ryals, M.; Choudhry, V. I.; Venton, B. J. Characterization of Spontaneous, Transient Adenosine Release in the Caudate-Putamen and Prefrontal Cortex. PLoS One 2014, 9 (1), e87165.

(136) Xu, Y.; Venton, B. J. Rapid Determination of Adenosine Deaminase Kinetics Using FastScan Cyclic Voltammetry. Phys. Chem. Chem. Phys. 2010, 12 (34), 10027-10032.

(137) Bath, B. D.; Michael, D. J.; Trafton, B. J.; Joseph, J. D.; Runnels, P. L.; Wightman, R. M. Subsecond Adsorption and Desorption of Dopamine at Carbon-Fiber Microelectrodes. Anal. Chem. 2000, 72 (24), 5994-6002.

(138) Keithley, R. B.; Takmakov, P.; Bucher, E. S.; Belle, A. M.; Owesson-White, C. A.; Park, J.; Wightman, R. M. Higher Sensitivity Dopamine Measurements with Faster-Scan Cyclic Voltammetry. Anal. Chem. 2011, 83 (9), 3563-3571.

(139) Ross, A. E.; Venton, B. J. Sawhorse Waveform Voltammetry for Selective Detection of Adenosine, ATP, and Hydrogen Peroxide. Anal. Chem. 2014, 86 (15), 7486-7493.

(140) Yuste, R. Neural Networks. Nat. Publ. Gr. 2015, 16 (8), 487-497.

(141) Stanley, G. B. Perspective Reading and Writing the Neural Code. Nat. Publ. Gr. 2013, 16 (3), 259-263.

(142) Cash, S. S.; Hochberg, L. R. Perspective The Emergence of Single Neurons in Clinical Neurology. Neuron 2015, 86 (1), 79-91.

(143) Fu, T.; Hong, G.; Zhou, T.; Schuhmann, T. G.; Viveros, R. D.; Lieber, C. M. Stable LongTerm Chronic Brain Mapping at the Single-Neuron Level. 2016, 13 (10).

(144) Hamel, E. J. O.; Grewe, B. F.; Parker, J. G.; Schnitzer, M. J. Review Cellular Level Brain Imaging in Behaving Mammals : An Engineering Approach. Neuron 2015, 86 (1), 140-159.

(145) Hanssen, B. L.; Siraj, S.; Wong, D. K. Y. Recent Strategies to Minimise Fouling in Electrochemical Detection Systems. Rev. Anal. Chem. 2016, 35 (1), 1-28.

(146) Roeser, J.; Alting, N. F. A.; Permentier, H. P.; Bruins, A. P.; Bischoff, R. Boron-Doped Diamond Electrodes for the Electrochemical Oxidation and Cleavage of Peptides. Anal. Chem. 2013, 85 (14), 6626-6632. 
(147) Wrona, M. Z.; Lemordant, D.; Lin, L.; Blank, C. L.; Dryhurst, G. Oxidation of 5Hydroxytryptamine and 5,7-Dihydroxytryptamine. A New Oxidation Pathway and Formation of a Novel Neurotoxin. J. Med. Chem. 1986, 29 (4), 499-505.

(148) Wrona, M. Z.; Dryhurst, G. Electrochemical Oxidation of 5-Hydroxytryptamine in Aqueous Solution at Physiological pH. Bioorg. Chem. 1990, 18 (3), 291-317.

(149) Yang, X.; Kirsch, J.; Olsen, E. V.; Fergus, J. W.; Simonian, A. L. Anti-Fouling PEDOT:PSS Modification on Glassy Carbon Electrodes for Continuous Monitoring of Tricresyl Phosphate. Sensors Actuators, B Chem. 2013, 177, 659-667.

(150) Singh, Y. S.; Sawarynski, L. E.; Dabiri, P. D.; Choi, W. R.; Andrews, A. M. Head-to-Head Comparisons of Carbon Fiber Microelectrode Coatings for Sensitive and Selective Neurotransmitter Detection by Voltammetry. Anal. Chem. 2011, 83 (17), 6658-6666.

(151) Muna, G. W.; Partridge, M.; Sirhan, H.; VerVaet, B.; Guerra, N.; Garner, H. Electrochemical Detection of Steroid Hormones Using a Nickel-Modified Glassy Carbon Electrode. Electroanalysis 2014, 26 (10), 2145-2151.

(152) Guo, Q.; Liu, D.; Zhang, X.; Li, L.; Hou, H.; Niwa, O.; You, T. Pd-Ni Alloy Nanoparticle/carbon Nanofiber Composites: Preparation, Structure, and Superior Electrocatalytic Properties for Sugar Analysis. Anal. Chem. 2014, 86 (12), 5898-5905.

(153) Yadav, S. K.; Agrawal, B.; Oyama, M.; Goyal, R. N. Graphene Modified Palladium Sensor for Electrochemical Analysis of Norepinephrine in Pharmaceuticals and Biological Fluids. Electrochim. Acta 2014, 125, 622-629.

(154) Mudrinić, T.; Mojović, Z.; Milutinović-Nikolić, A.; Banković, P.; Dojčinović, B.; Vukelić, N.; Jovanović, D. Beneficial Effect of $\mathrm{Ni}$ in Pillared Bentonite Based Electrodes on the Electrochemical Oxidation of Phenol. Electrochim. Acta 2014, 144, 92-99.

(155) Zheng, M.; Zhou, Y.; Chen, Y.; Tang, Y.; Lu, T. Electrochemical Behavior of Dopamine in the Presence of Phosphonate and the Determination of Dopamine at Phosphonate Modified Zirconia Films Electrode with Highly Antifouling Capability. Electrochim. Acta 2010, 55 (16), 4789-4798.

(156) Takmakov, P.; Zachek, M. K.; Keithley, R. B.; Walsh, P. L.; Donley, C.; McCarty, G. S.; Wightman, R. M. Carbon Microelectrodes with a Renewable Surface. Anal. Chem. 2010, 82 (5), 2020-2028.

(157) Kiran, R.; Scorsone, E.; de Sanoit, J.; Arnault, J.-C.; Mailley, P.; Bergonzo, P. Boron Doped Diamond Electrodes for Direct Measurement in Biological Fluids: An In Situ Regeneration Approach. J. Electrochem. Soc. 2013, 160 (1), H67-H73.

(158) Tang, L.; Thevenot, P.; Hu, W. Surface Chemistry Influences Implant Biocompatibility. Curr. Top. Med. Chem. 2008, 8 (4), 270-280.

(159) Sarada, B. V. Electrochemical Characterization of Highly Boron-Doped Diamond Microelectrodes in Aqueous Electrolyte. J. Electrochem. Soc. 1999, 146 (4), 1469.

(160) Xu, J.; Chen, Q.; Swain, G. M. Anthraquinonedisulfonate Electrochemistry: A Comparison of Glassy Carbon, Hydrogenated Glassy Carbon, Highly Oriented Pyrolytic Graphite, and Diamond Electrodes. Anal. Chem. 1998, 70 (15), 3146-3154.

(161) Patel, A. N.; Tan, S.; Miller, T. S.; Macpherson, J. V.; Unwin, P. R. Comparison and Reappraisal of Carbon Electrodes for the Voltammetric Detection of Dopamine. Anal. 
Chem. 2013, 85 (24), 11755-11764.

(162) Patel, J.; Radhakrishnan, L.; Zhao, B.; Uppalapati, B.; Daniels, R. C.; Ward, K. R.; Collinson, M. M. Electrochemical Properties of Nanostructured Porous Gold Electrodes in Biofouling Solutions. Anal. Chem. 2013, 85 (23), 11610-11618.

(163) Harreither, W.; Trouillon, R.; Poulin, P.; Neri, W.; Ewing, A. G.; Safina, G. Cysteine Residues Reduce the Severity of Dopamine Electrochemical Fouling. Electrochim. Acta 2016, 210, 622-629.

(164) Liu, X.; Zhang, M.; Xiao, T.; Hao, J.; Li, R.; Mao, L. Protein Pretreatment of Microelectrodes Enables in Vivo Electrochemical Measurements with Easy Precalibration and InterferenceFree from Proteins. Anal. Chem. 2016, 88 (14), 7238-7244.

(165) Chen, C.-H.; Luo, S.-C. Tuning Surface Charge and Morphology for the Efficient Detection of Dopamine under the Interferences of Uric Acid, Ascorbic Acid, and Protein Adsorption. ACS Appl. Mater. Interfaces 2015, 7 (39), 21931-21938.

(166) Baldrich, E.; Muñoz, F. X. Carbon Nanotube Wiring: A Tool for Straightforward Electrochemical Biosensing at Magnetic Particles. Anal. Chem. 2011, 83 (24), 9244-9250.

(167) Taylor, I. M.; Robbins, E. M.; Catt, K. A.; Cody, P. A.; Happe, C. L.; Cui, X. T. Enhanced Dopamine Detection Sensitivity by PEDOT/graphene Oxide Coating on in Vivo Carbon Fiber Electrodes. Biosens. Bioelectron. 2016, 0-1.

(168) Cahill, P. S.; Walker, Q. D.; Finnegan, J. M.; Mickelson, G. E.; Travis, E. R.; Wightman, R. M. Microelectrodes for the Measurement of Catecholamines in Biological Systems. Anal. Chem. 1996, 68 (18), 3180-3186.

(169) Xue, Q.; Kato, D.; Kamata, T.; Umemura, S.; Hirono, S.; Niwa, O. Electron Cyclotron Resonance-Sputtered Nanocarbon Film Electrode Compared with Diamond-like Carbon and Glassy Carbon Electrodes as Regards Electrochemical Properties and Biomolecule Adsorption. Jpn. J. Appl. Phys. 2012, 51 (9).

(170) Kato, D.; Komoriya, M.; Nakamoto, K.; Kurita, R.; Hirono, S.; Niwa, O. Electrochemical Determination of Oxidative Damaged DNA with High Sensitivity and Stability Using a Nanocarbon Film. Anal. Sci. 2011, 27 (7), 703.

(171) Duran, B.; Brocenschi, R. F.; France, M.; Galligan, J. J.; Swain, G. M. Electrochemical Activation of Diamond Microelectrodes: Implications for the in Vitro Measurement of Serotonin in the Bowel. Analyst 2014, 139, 3160-3166.

(172) Stoytcheva, M.; Zlatev, R.; Gochev, V.; Velkova, Z.; Montero, G.; Beleño, M. T. Amperometric Biosensors Precision Improvement. Application to Phenolic Pollutants Determination. Electrochim. Acta 2014, 147, 25-30.

(173) Agnesi, F.; Tye, S. J.; Bledsoe, J. M.; Griessenauer, C. J.; Kimble, C. J.; Sieck, G. C.; Bennet, K. E.; Garris, P. A.; Blaha, C. D.; Lee, K. H. Wireless Instantaneous Neurotransmitter Concentration System-Based Amperometric Detection of Dopamine, Adenosine, and Glutamate for Intraoperative Neurochemical Monitoring. J. Neurosurg. 2009, 111 (4), 701-711.

(174) Schmidt, A. C.; Dunaway, L. E.; Roberts, J. G.; McCarty, G. S.; Sombers, L. A. Multiple Scan Rate Voltammetry for Selective Quantification of Real-Time Enkephalin Dynamics. Anal. Chem. 2014, 86 (15), 7806-7812. 
Chapter 2: Carbon Nanotubes Grown on Metal Microelectrodes for the Detection of Dopamine 


\section{Abstract}

Microelectrodes modified with carbon nanotubes (CNTs) are useful for the detection of neurotransmitters because the CNTs enhance sensitivity and have electrocatalytic effects. CNTs can be grown on carbon fiber microelectrodes (CFMEs) but the intrinsic electrochemical activity of carbon fibers makes evaluating the effect of CNT enhancement difficult. Metal wires are highly conductive and many metals have no intrinsic electrochemical activity for dopamine so we investigated CNTs grown on metal wires as microelectrodes for neurotransmitter detection. In this work, we successfully grew CNTs on niobium substrates for the first time. Instead of planar metal surface, metal wires with a diameter of only $25 \mu \mathrm{m}$ were used as CNT substrates, which have potential for in tissue applications due to their minimal tissue damage and high spatial resolution. Scanning electron microscopy shows that aligned CNTs are grown on metal wires after chemical vapor deposition. Using fast-scan cyclic voltammetry, CNT-coated niobium (CNT-Nb) microelectrodes exhibit higher sensitivity and lower $\Delta \mathrm{E}_{\mathrm{p}}$ value compared to CNTs grown on carbon fibers or other metal wires. The limit of detection for dopamine at CNT-Nb microelectrodes is $11 \pm 1 \mathrm{nM}$, which is approximately two fold lower than bare CFMEs. Adsorption processes were modeled with a Langmuir isotherm and detection of other neurochemicals was also characterized, including ascorbic acid, DOPAC, serotonin, adenosine, and histamine. CNT-Nb microelectrodes were used to monitor stimulated dopamine release in anesthetized rats with high sensitivity. This study demonstrates that CNT-grown metal microelectrodes, especially CNTs grown on $\mathrm{Nb}$ microelectrodes, are useful for monitoring neurotransmitters. This paper was published in Analytical Chemistry (Anal. Chem., 2015, 88 (1), 645-652). 


\subsection{Introduction}

CNT-modified electrodes have been widely used for the detection of biomolecules because of their unique properties including large active surface area, high conductivity, fast electron transfer kinetics and biocompatibility..$^{1,2}$ These properties lead to reduced overpotential, minimal electrode fouling, and increased sensitivity and selectivity. ${ }^{3,4}$ CNTs are especially attractive for making smaller electrodes because the high surface-area-to-volume ratio results in a large electroactive surface area for the adsorption of biomolecules. ${ }^{5}$ A popular method to deposit CNT films onto microelectrodes is to dip CFMEs into CNT suspension or CNT/polymer composite solution. ${ }^{6-9}$ However, CNTs are randomly distributed throughout the CNT films during the dip coating process. Therefore, most of the area exposed to the analyte solution is the sidewall of the CNTs, but the ends of the CNTs are more likely to be the most electrochemically active sites. ${ }^{10-}$ ${ }^{13}$ Moreover, large CNT agglomerations are easily formed which cause high noise and the cumbersome fabrication procedure reduces the reproducibility. ${ }^{7}$

Previous studies have shown that vertically-aligned CNTs on a microelectrode substrate are better for detecting neurotransmitters, such as dopamine. ${ }^{14}$ One strategy is to chemically selfassemble vertically-aligned CNTs on substrates with a solution deposition method. Our group developed single walled carbon nanotube (SWCNT) forest-modified CFMEs for rapid and sensitive detection of neurotransmitters using fast-scan cyclic voltammetry (FSCV). ${ }^{15}$ An alternative strategy is to directly grow CNTs in an aligned manner through chemical vapor deposition (CVD). Recently, Xiang et al. used as-synthesized, vertically-aligned carbon nanotube sheathed CFs (VACNT-CFs) for the detection of dopamine and ascorbate in vivo. ${ }^{16}$ The VACNTCFs microelectrodes exhibited promising electrochemical performance. However, since carbon fiber (CF) is electrochemically active towards dopamine, the CF substrates limit the studies of the properties of CNT coating. In comparison, metal substrates with CNT coating would have several benefits. First, although gold ${ }^{17}$ and platinum ${ }^{18}$ are active to dopamine, many other metals (e.g $\mathrm{Nb}$, Ta, Mo, W, Pd, Ti, and stainless steel used in this paper) lack electrochemical reactivity to 
dopamine, which enables the study of the interaction of dopamine with CNTs without the convolution of possible substrate reactivity. Second, the inherently low conductivity of $\mathrm{CF}^{10}$ may limit the overall conductivity of sensors, while metals have higher conductivity. Third, the electrochemical properties of the CF core vary with different waveforms and can affect the electrochemical properties. ${ }^{19}$ Therefore, a metal substrate which lacks reactivity to dopamine, has high intrinsic conductivity, and relatively stable electrochemistry may avoid these issues. Although successful growth of CNTs on several metal substrates has been reported, ${ }^{20-26}$ CNTs have not been grown on niobium $(\mathrm{Nb})$ substrate. In addition, all previous studies of CNT growth on metals have been on flat substrates and not on the cylindrical metal wires that would be needed for implantable electrochemical micro-sensors.

In this study, we explored the use of CNT-grown metal microelectrodes for enhanced neurotransmitter detection. The CNT-grown metal and CFMEs were fabricated by CVD and characterized with scanning electron microscopy and Raman spectroscopy. These are the first studies to grow CNTs on Nb substrates or on small diameter metal wires, instead of a planar metal surfaces such as foils, which allows them to be implanted in tissue with minimal damage and high spatial resolution. ${ }^{27}$ CNTs grown on $\mathrm{Nb}$ were short and dense, and CNT-Nb microelectrodes exhibited better electrochemical response to dopamine using FSCV compared to CNTs grown on other metals or CFs. Moreover, the CNT-Nb microelectrodes have been tested for the electrochemical response to ascorbic acid, DOPAC (3,4 dihydroxyphenylacetic acid, dopamine metabolite), serotonin, adenosine, and histamine. The CNT-Nb microelectrodes were used to detect stimulated dopamine release in anesthetized rats and exhibited high sensitivity with rapid measurements in vivo. Electrophysiology studies often use arrays of metal wires and future experiments could investigate making arrays of the CNTs on metal wires for multiplexed electrochemical experiments. 


\subsection{Experimental Section}

\subsubsection{Solutions}

Dopamine, ascorbic acid, DOPAC, adenosine, 5-hydroxytryptamine (serotonin), and histamine were purchased from Sigma-Aldrich (St. Louis, MO). Ten millimolar (10 mM) stock solutions of the analytes were prepared in $\mathrm{HClO}_{4}$ and were diluted daily to the desired concentration in phosphate-buffered saline (PBS) (15 mM tris(hydroxymethyl)aminomethane, $3.25 \mathrm{mM} \mathrm{KCl}, 140 \mathrm{mM} \mathrm{NaCl}, 1.2 \mathrm{mM} \mathrm{CaCl}_{2}, 1.25 \mathrm{mM} \mathrm{NaH}_{2} \mathrm{PO}_{4}, 1.2 \mathrm{mM} \mathrm{MgCl}_{2}$, and $2.0 \mathrm{mM}$ $\mathrm{Na}_{2} \mathrm{SO}_{4}$, with the $\mathrm{pH}$ adjusted to 7.4).

\subsubsection{Synthesis of CNTs coated metal wires and carbon fibers}

Carbon fibers (T650-35, Cytec, Woodland Park, NJ) and metal wires including tantalum, niobium, molybdenum, tungsten, stainless steel, titanium, and palladium (diameter of 0.001", ESPI Metals, Ashland, OR) were used as electrode substrates. CNTs were grown in an aligned manner through CVD after a solid-phase catalyst was deposited on the substrate surface ${ }^{24,28} \mathrm{~A}$ thin film of $\mathrm{Al}_{2} \mathrm{O}_{3}(30 \mathrm{~nm})$ followed by a film of Fe catalyst $(1 \mathrm{~nm})$ were deposited onto the metal wires $(25 \mu \mathrm{m})$ or CFs $(7 \mu \mathrm{m})$ using electron beam physical vapor deposition (Angstrom Engineering, Kitchener, Ontario, Canada). Since electron beam deposition is "line-of-sight" dependent, only one side of the substrate was coated with buffer layer and catalyst. As a result, the microelectrodes were half coated with CNT arrays. In a quartz tube CVD reactor, the $\mathrm{Al}_{2} \mathrm{O}_{3}$ Fe coated CFs and metal wires were degassed in vacuum and the temperature of the reactor was slowly ramped up to $700^{\circ} \mathrm{C}$ and held for 10 min with a flow mixture of Argon (2000 sccm) and $\mathrm{H}_{2}$ (200 sccm). Then, ethylene (10 sccm) was introduced through the quartz tube for 5 min to grow the CNTs. 


\subsubsection{Microelectrode preparation}

A CNT-grown wire or CF, 1-2 cm long, was inserted into a polyimide coated fused-silica capillary $(45 \mu \mathrm{m}$ inner diameter (ID) $\times 90 \mu \mathrm{m}$ outer diameter (OD), Polymicro Technologies, Phoenix, AZ) while submerged in 2-propanol, to reduce friction and ease insertion. The solvent was allowed to fully evaporate from inside of the capillary before the metal wire was sealed into the capillary with 5 min epoxy Loctite (Henkel Corporation, Westlake $\mathrm{OH}$ ) and was allowed to fully cure for $24 \mathrm{~h}$. The polyimide capillary was then inserted and epoxied in a $0.68 \mathrm{~mm} \mathrm{ID} \times 1.2 \mathrm{~mm}$ OD glass capillary that had previously been pulled into a glass pipet and cut to have an opening diameter of $\sim 100 \mu \mathrm{m}$.

\subsubsection{Surface characterization}

Scanning electron microscope (SEM) images were taken on Merlin field emission SEM (Zeiss, Thornwood, NY) with a secondary electron detector using an accelerating voltage of $2 \mathrm{kV}$ and a working distance of $5.3 \mathrm{~mm}$. Transmission electron microscope (TEM) images were taken on a Libra 120 (Zeiss, Thornwood, NY). Raman spectroscopy measurements were performed with a Renishaw 100 confocal micro-Raman system (Renishaw, Hoffman Estates, IL) with a 1800 lines/mm diffraction grating, $532 \mathrm{~nm}$ laser focused to a spot size of about $2 \mu \mathrm{m}$ through a 100x objective, and a Peltier-cooled charge-coupled device detector.

Surface areas were estimated by either integrating the background current and multiplying by time or using the background current at $0.25 \mathrm{~V}$. These currents were divided by specific capacitance $\left(24 \mu \mathrm{F} / \mathrm{cm}^{2}\right.$, a standard capacitance for glassy carbon $\left.{ }^{10}\right)$ times scan rate to determine surface areas. Both methods gave similar areas. Since CNTs are only half coated on metal/carbon fiber substrates, the capacitive charging current used for surface area calculation was performed by the difference of background current of CNT-metal electrode minus half of the 
background current of bare metal electrodes. While the specific capacitance might vary by CNT material, it allows a rough estimation of surface area.

\subsubsection{Electrochemistry}

FSCV was performed with a ChemClamp potentiometer (Dagan, Minneapolis, MN, with 1 mega Ohm Chem Clamp Headstage). The waveform was generated and the data was collected using a High Definition Cyclic Voltammetry breakout box (UNC Chemistry Department, Electronics Design Facility) and PCle-6363 computer interface cards (National Instruments, Austin, TX). Electrodes were backfilled with $1 \mathrm{M} \mathrm{KCl}$ and a silver wire was inserted to connect the electrode to the potentiostat probe. The typical triangular waveform swept the applied potential from $-0.4 \mathrm{~V}$ to $1.3 \mathrm{~V}$ (except where noted) at $400 \mathrm{~V} / \mathrm{s}$ versus an $\mathrm{Ag} / \mathrm{AgCl}$ reference electrode, at a scan frequency of $10 \mathrm{~Hz}$. Data collection was computer-controlled by the HDCV analysis software program (University of North Carolina, Chapel Hill).

Electrodes were tested using a flow-injection system, as previously described. ${ }^{29}$ Analyte was injected for 5 seconds and current versus time traces were obtained by integrating the current in a $100 \mathrm{mV}$ window centered at the oxidation peak for each cyclic voltammogram (CV). Background-subtracted CVs were calculated by subtracting the average of 10 background scans, taken before the compound was injected, from the average of five CVs recorded after the analyte bolus was injected.

\subsubsection{In vivo measurements}

Male Sprague-Dawley rats (250-350 g) purchased from Charles River were housed in a vivarium and given food and water ab libitum. All experiments were approved by the Animal Care and Use Committee of the University of Virginia. The rat was anesthetized with urethane (Sigma 
Aldrich) (1.5 mg/kg i.p.), the scalp shaved, and $0.25 \mathrm{~mL}$ bupivicaine $(0.25 \%$ solution, Sensorcaine, MPF, APP Pharmaceuticals, LLC; Schaumburg, IL) given subcutaneously. The working electrode was implanted in the caudate putamen (in mm from bregma: $\mathrm{AP}+1.2, \mathrm{ML}+2.0$, and $\mathrm{DV}-4.5$ to -5.0 ), the stimulating electrode in the substantia nigra ( $A P-5.4, M L+1.2$, and $D V-8.0$ ), and the $\mathrm{Ag} / \mathrm{AgCl}$ reference electrode on the contralateral side of the brain. The DV placement of the stimulating electrode was adjusted downward until a robust dopamine signal was measured. After implantation in the brain, the FSCV waveform was applied to the CNT-Nb microelectrode for 30 min to allow the electrode to stabilize and the brain to recover. Stimulated release was electrically evoked using biphasic stimulation pulses (300 $\mu \mathrm{A}, 12-120$ pulses, $60 \mathrm{~Hz})$.

\subsubsection{Statistics}

All values are given as mean \pm standard error of the mean (SEM) for $n$ number of electrodes and all error bars are given as SEM. Unpaired t-tests were performed to compare properties between two groups. A one-way ANOVA with Bonferonni post-tests was used to compare effects among multiple groups. All statistics were performed in GraphPad Prism (GraphPad Software,Inc., La Jolla, CA). SEM and TEM images were processed using ImageJ (Rasband, W.S., ImageJ, National Institutes of Health, Bethesda, MD, U.S.A.).

\subsubsection{Langmuir isotherm modeling}

We used a Langmuir adsorption isotherm (Eq. 1) to model the adsorption and desorption process kinetics of dopamine.

$$
\frac{\Gamma_{D A}}{\Gamma_{S}}=\frac{\beta_{D A} a_{D A}^{b}}{1+\beta_{D A} a_{D A}^{b}}
$$

$\Gamma_{\mathrm{DA}}$ is the amount of dopamine adsorbed on the electrode, $\Gamma_{\mathrm{s}}$ is the saturated amount of dopamine that can adsorb on the electrode, $\beta_{\mathrm{DA}}$ is the thermodynamic equilibrium constant (unitless) for 
dopamine, and $\mathrm{a}_{\mathrm{DA}}^{\mathrm{b}}$ is the activity of dopamine in bulk solution at equilibrium. The percent surface coverage, $\Gamma_{\mathrm{DA}} / \Gamma_{\mathrm{s}}$, can be expressed by the ratio of the oxidation current of dopamine to the theoretical saturated oxidation current which is the plateau of the fitting curve. The activity is related to its molar concentration $\left(C_{D A}\right)$ by the following Equation $2 \cdot 2^{30}$

$$
a_{D A}=\left(\gamma_{D A} \cdot C_{D A}\right) \cdot\left(1 L \cdot \mathrm{mol}^{-1}\right) \quad \text { (Eq. 2.2) }
$$

$\gamma_{D A}$ is the activity coefficient of dopamine in bulk solution at the adsorption equilibrium. For a charged adsorbate solution at high concentration, the effect of the activity coefficients must be taken into account because charged adsorbates are governed by ionic interactions. ${ }^{31}$ According to the Debye-Huckel law:

$$
\log \gamma_{D A}=-A z^{2} I^{\frac{1}{2}} \text { (Eq. 2.3) }
$$

$\gamma_{D A}$ is a function of the ionic strength $(I)$ of the solute and the charge carried by each solute $(z), A$ is a constant that depends on temperature and is about 0.51 for water at $25^{\circ} \mathrm{C} .{ }^{32}$ Thus, $\gamma_{\mathrm{DA}}$ for dopamine in PBS buffer is 0.63 at room temperature. ${ }^{33} \beta_{D A}$ can be used to calculate the adsorption Gibbs free energy of dopamine.

$$
\Delta \bar{G}_{D A}^{o}=-R T \ln \beta_{D A} \quad \text { (Eq. 2.4) }
$$




\subsection{Results and Discussion}

\subsubsection{Characterization of CNTs grown on metal wires and carbon fibers}

CVD allows direct growth of CNTs on substrates; however, no study had grown CNTs on $\mathrm{Nb}$ metal or on small diameter cylindrical metal wires. We optimized CNT growth on metal wires for the use as microelectrodes. Figure 2.1 shows scanning electron microscope (SEM) images of bare $\mathrm{Nb}$ (Fig. 2.1A) and Ta (Fig. 2.1B) wires as well as a CF (Fig. 2.1C), and the same substrates after CNT growth (Fig. 2.1 D-F). The CNTs (multi-walled) grown on Nb are short, dense, and aligned, compared to the CNTs grown on Ta and CFs, which are longer and more randomly oriented. Since the end caps of the CNTs would be open due to the applied voltage ${ }^{34}$, the ends would have more $s p^{3}$ hybridized, edge-plane carbons that can be oxidized to provide functional groups $^{10}$. The short, dense CNT bundles on the $\mathrm{Nb}$ would have more functionalized edge plane sites exposed compared to the more diffuse CNTs on CFs and Ta, where more side walls would be exposed to the analyte.
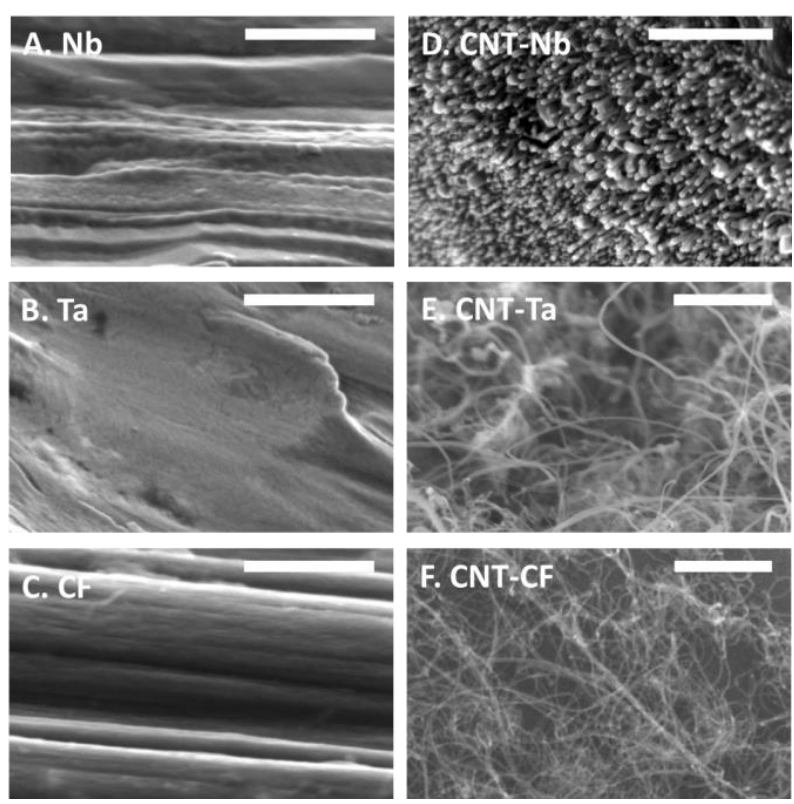

Figure 2.1: SEM images of bare metals and metals with CNT growth. A. bare niobium, B. bare tantalum, C. bare carbon fiber, D. CNT-grown niobium, E. CNT-grown tantalum, and F. CNTgrown CF. Scale bar: 500nm. 
The variety in CNT morphology grown on different metallic substrates might result from the interaction of $\mathrm{Al}_{2} \mathrm{O}_{3}$ buffer layer with the substrate or different properties of the metals. $\mathrm{Al}_{2} \mathrm{O}_{3}$ was used as a catalyst support buffer layer to enhance CNT growth by inhibiting the diffusion of the catalyst material into the substrate upon heating. ${ }^{35}$ However, the $\mathrm{Al}_{2} \mathrm{O}_{3}$ buffer layer has a different grain size on different substrates after heating, due to surface energy or wettability, ${ }^{36-38}$ which leads to different CNT nucleation density. ${ }^{39}$ Another possible reason for the varied CNT morphology on different substrates is the amount of hydrogen absorbed in the metal substrates, which could affect the microstructure and mechanical properties of the resulting CNT gowth. ${ }^{40}$ Among transition metals, VB group metals are good hydrogen storage substrates. ${ }^{41-43}$ Therefore, the more aligned and consistent CNT growth on $\mathrm{Nb}$ and $\mathrm{Ta}$ might be due to hydrogen release that helps maintain the activation of iron catalysts.

To further characterize the CNT surface, Raman spectra of CNTs grown on metal wires and CF substrates were compared (Fig. 2.2). The ratio of $D / G$ peaks reveals the $s p^{3}$ hybridized content of the carbon film. ${ }^{44}$ The D/G ratio for CNT-Nb, CNT-Ta, and CNT-CF are $2.2 \pm 0.1(\mathrm{n}=$ 5), $1.8 \pm 0.2(n=5)$, and $1.9 \pm 0.6(n=5)$, respectively. The ratio of the intensity of these peaks is often used as an indicator of the quality of CNTs and these multi-walled CNTs are defect rich. ${ }^{45}$ The $\mathrm{D} / \mathrm{G}$ ratio of CNTs grown on $\mathrm{Nb}$ is significantly larger than on Ta (unpaired t-test, $\mathrm{p} \leq 0.05$ ), which demonstrates CNTs on Nb are more defect rich. The small standard errors observed indicate that the $D / G$ ratio was consistent between electrodes. 

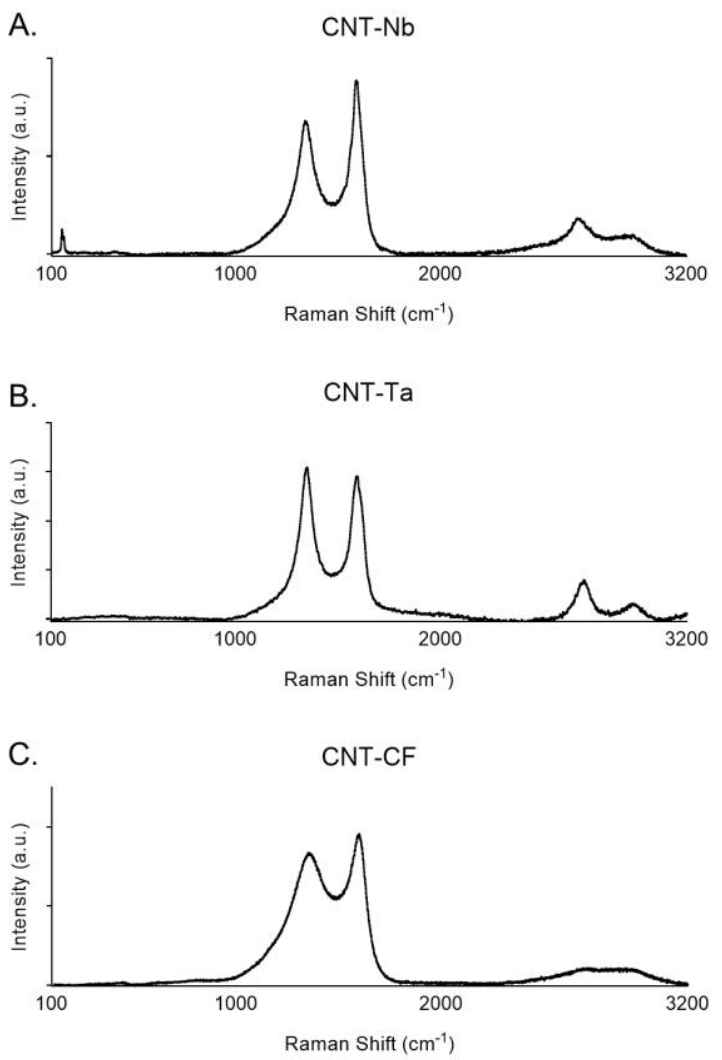

Figure 2.2: Raman spectrum of CNT grown on the surface of (A) Nb, (B) Ta and (C) CF wires.

\subsubsection{FSCV of dopamine at bare metal wire microelectrodes and CFMEs}

To investigate the electrochemical performance of the substrate materials, cylindrical microelectrodes were made of metal wires and CFs. Figure 2.3A-C shows the background current measured in PBS buffer at bare $\mathrm{Nb}, \mathrm{Ta}$, and CF electrodes with similar lengths ( 70-100 $\mu \mathrm{m})$. The capacitive currents arising from the electrical double layer charging are small, around $300 \mathrm{nA}$ for $\mathrm{Nb}$ and $100 \mathrm{nA}$ for Ta metal wires. The square shape background for $\mathrm{Nb}$ and Ta metal wires reveals good polarizability. ${ }^{10}$ In contrast, larger background currents at CFME can be attributed to surface functional groups as well as capacitive charging. ${ }^{46}$ 

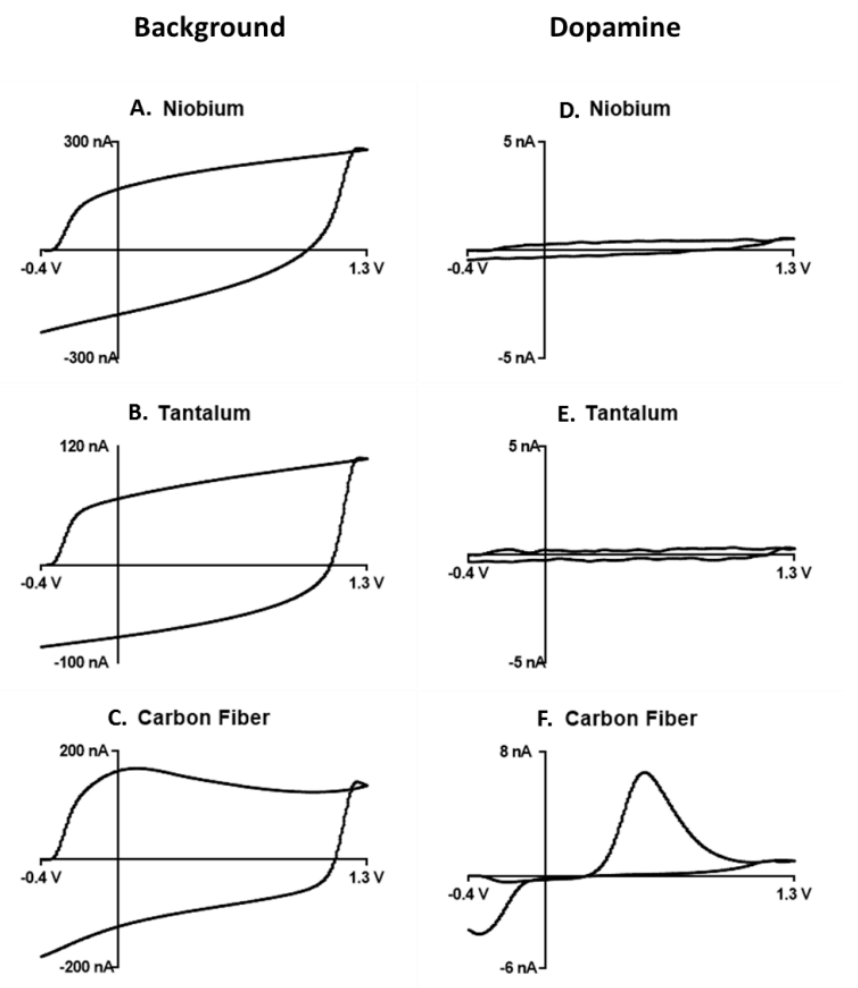

Figure 2.3: Electrochemical response of bare metal or carbon fibers with scan rate of $400 \mathrm{~V} / \mathrm{s}$ and repetition frequency of $10 \mathrm{~Hz}$. Background current in PBS buffer solution for A. niobium, B. tantalum, C. carbon-fiber microelectrodes. Right column: Background-subtracted cyclic voltammograms for $1 \mu \mathrm{M}$ dopamine at bare D. niobium, E. tantalum, and F. carbon-fiber microelectrodes.

Figures 2.3D-F shows the background subtracted cyclic voltammograms (CVs) of $1 \mu \mathrm{M}$ dopamine at bare $\mathrm{Nb}, \mathrm{Ta}$, and $\mathrm{CFs}$. $\mathrm{Nb}$ and $\mathrm{Ta}$ are not electrochemically active for dopamine and show no faradaic peaks. Therefore, any dopamine signal at CNT-Nb or CNT-Ta microelectrodes will arise from the CNTs. CFMEs have a robust signal for dopamine (Fig. 2.3F) and are widely used as the standard electrode material in the field of in vivo voltammetry.

\subsubsection{FSCV of dopamine at CNT-metal wire microelectrodes and CNT-CFMEs}

Figure 2.4 shows the electrochemical response of CNT-grown Nb and Ta microelectrodes and CNT-grown CFMEs. The background charging currents for the CNT-Nb and CNT-Ta electrodes (Fig. 2.4A-B) are significantly larger than for the bare metals (Fig. 2.3A-B), indicating 
substantial CNT growth. For CNTs grown on Nb and Ta wires, background-subtracted CVs for 1 $\mu \mathrm{M}$ dopamine (Fig. 2.4D-E) show Faradaic peaks which were not present for bare wires (Fig. 2.3D-E). Faradaic peaks for dopamine are also observed at CNT-CF microelectrodes, but the contributions of the CNTs vs CF to the signal are harder to distinguish. Moreover, dopamine oxidation is more reversible at CNT-Nb microelectrodes than that for CFMEs, which can be observed in the CVs.

\section{Background}

A. CNT-Nb

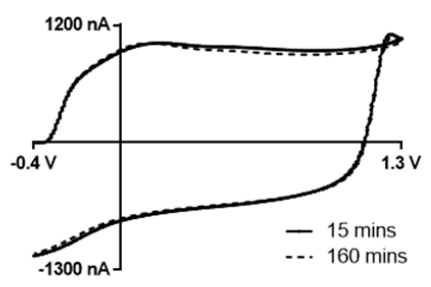

B. CNT-Ta

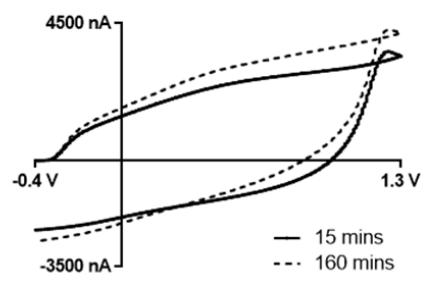

C. CNT-CF

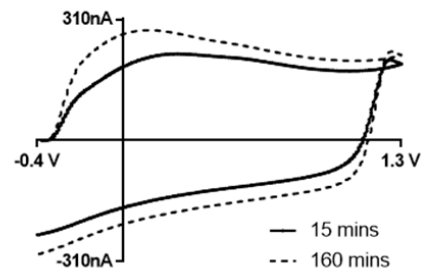

Dopamine

D. $\mathrm{CNT}-\mathrm{Nb}$

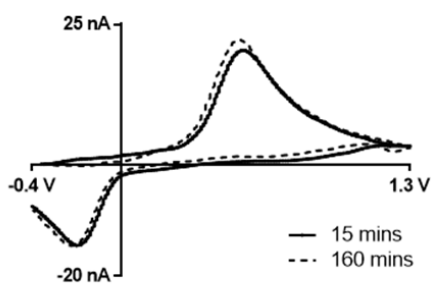

E. CNT-Ta

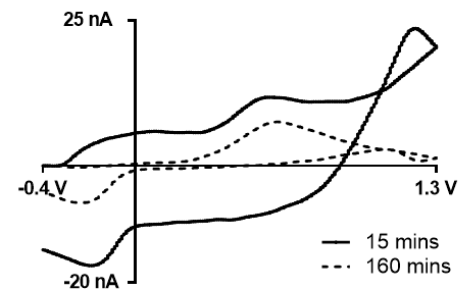

F. CNT-CF

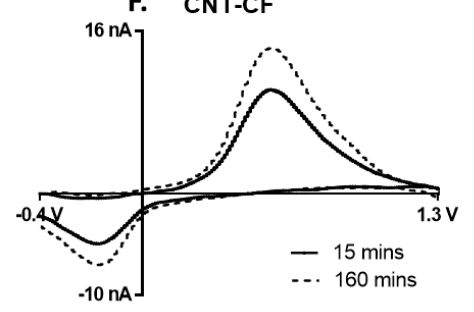

Figure 2.4: Comparison of electrochemical response at CNT-grown niobium, tantalum and carbon fiber microelectrodes. Background current at A. CNT-Nb, B. CNT-Ta, and C. CNT-CF, and background-subtracted cyclic voltammograms for $1 \mu \mathrm{M}$ dopamine at D. CNT-Nb, E. CNT-Ta, and F. CNT-CF microelectrodes. The solid lines indicate the measurements taken after 15 min equilibration and the dashed lines indicate the measurements after 160 min equilibration in PBS buffer solution using a waveform of -0.4 to $1.3 \mathrm{~V}$ and back at $400 \mathrm{~V} / \mathrm{s}, 10 \mathrm{~Hz}$.

Equilibration at carbon-based electrodes is required since the carbon surface can change with the application of the triangle waveform. Equilibration with a fast-scan triangle waveform 
$(400 \mathrm{~V} / \mathrm{s},-0.4 \mathrm{~V}-1.3 \mathrm{~V}$ vs $\mathrm{Ag} / \mathrm{AgCl})$ mildly etches the carbon surface and introduces more oxygen containing functional groups as active adsorption sites for dopamine. ${ }^{46}$ For CNT-grown microelectrodes, the background (Fig. 2.4A-C) and response to $1 \mu \mathrm{M}$ dopamine (Fig. 2.4D-F) were measured at two equilibration time points: waveform application for $15 \mathrm{~min}$ and $160 \mathrm{~min}$. Equilibration time mattered little for CNT-Nb microelectrodes, as the response to dopamine and background current were similar for both time points (Fig. 2.5A). In contrast, CNT-Ta and CNTCF required a longer equilibration time (Fig. 2.5B-C). The shorter equilibration time might be due to abundant defects sites at CNT grown on $\mathrm{Nb}$, which could be oxygen-functionalized faster by electrochemical activation. ${ }^{47}$ The ends of CNTs grown on $\mathrm{Nb}$ are likely open, especially after continuous scanning with the $1.3 \mathrm{~V}$ triangle waveform, while the main sources of adsorption sites at CNT-Ta and CNT-CF are probably defects on sidewalls. The CNT-Nb microelectrode had no significant change in peak oxidative current for dopamine over 4 hours indicating the electrodes are stable over the typical time length of a biological experiment (Fig. 2.6).

A. CNT-Nb Microelectrode

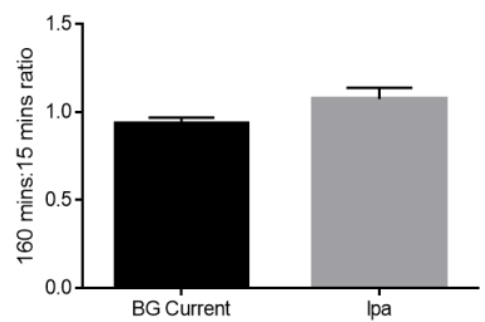

B. CNT-Ta Microelectrode

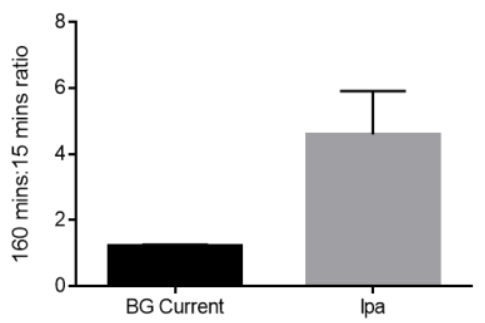

C. CNT-CF Microelectrode

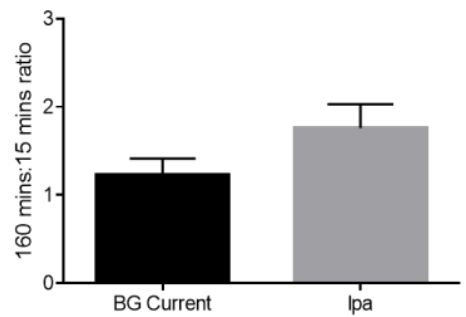

Figure 2.5: Effect of equilibration at (A) CNT-Nb, (B) CNT-Ta, and (C) CNT-CF microelectrodes $(n=4)$. Values given are the ratio of the current at 160 mins of equilibration vs 15 mins of equilibration. The black bar is the ratio of background current at $0.25 \mathrm{~V}$ in PBS buffer, giving an indication of changes in background current. The gray bar is the ratio of oxidation currents for 1 $\mu \mathrm{M}$ dopamine. Ratios greater than 1 indicate that the peak increased in size during equilibration. 


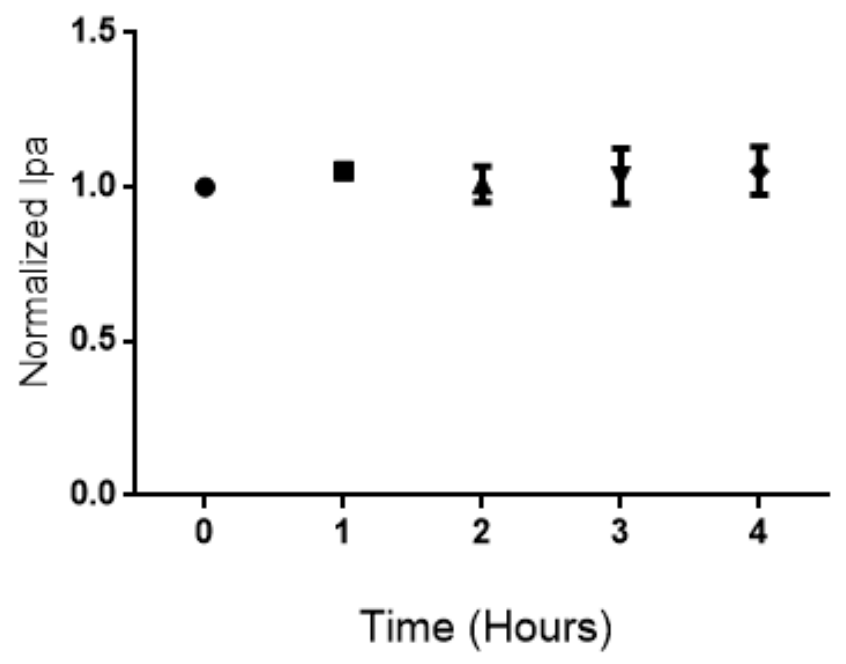

Figure 2.6: CNT-Nb microelectrodes stability experiment. Measurements of $1 \mu \mathrm{M}$ dopamine were taken over a $4 \mathrm{~h}$ time period at CNT-Nb microelectrodes, with a scan rate of $400 \mathrm{~V} / \mathrm{s}$ and sampling repetition rate of $10 \mathrm{~Hz}(\mathrm{n}=5$, error bar indicates standard error of mean).

To compare the sensitivity of electrodes to dopamine, currents were corrected for surface area (based on their capacitive charging currents), since the metal wires are $25 \mu \mathrm{m}$ diameter while the CFME is $7 \mu \mathrm{m}$ diameter. As shown in Table 2.1, the current density at CNT-Nb microelectrodes for $1 \mu \mathrm{M}$ dopamine is $197 \pm 16 \mathrm{pA} / \mu \mathrm{m}^{2}$, which is significantly larger than the current density at CNT-Ta, CNT-CF or CFMEs (one way-ANOVA Bonferonni post-test, $p<0.0005$, $n=5$ ). Current density at CFMEs after CNT growth is lower than bare CFMEs, indicating that much of CNT grown on the CF substrate is not as electrochemically active as CF to dopamine. Because of the spaghetti-like structure, not all of the CNTs on the CF may be available for electron transfer, but adding CNTs adds to the background current and the noise. The limit of detection (LOD) is $11 \pm 1 \mathrm{nM}(\mathrm{S} / \mathrm{N}=3)$ for dopamine at CNT-Nb microelectrodes, which is significantly lower than that of CNT-Ta, CNT-CF as well as CFMEs (one way-ANOVA Bonferonni post-test, $p$ $<0.005, n=5$ ). Therefore, with higher sensitivity and better LOD than CFMEs, CNT-Nb electrodes have a promising electrochemical performance for dopamine detection. 
Table 2.1: Average $\Delta \mathrm{E}_{\mathrm{p}}$, current density, and limit of detection for $1 \mu \mathrm{M}$ dopamine at CNT-grown microelectrodes and CFMEs.

$\begin{array}{lccc}\text { Electrodes } & \Delta \mathrm{E}_{\mathrm{p}}(\mathrm{V}) & \text { Current Density }\left(\mathrm{pA} / \boldsymbol{\mu m}^{2}\right) & \text { LOD }(\mathrm{nM}) \\ \text { CNT-Nb } & 0.73 \pm 0.03 & 197 \pm 16 & 11 \pm 1 \\ \text { CNT-Ta } & 0.87 \pm 0.01 & 82 \pm 10 & 91 \pm 27 \\ \text { CNT-CF } & 0.81 \pm 0.03 & 100 \pm 25 & 46 \pm 10 \\ \text { CFMEs } & 0.67 \pm 0.01 & 135 \pm 24 & 19 \pm 4\end{array}$

All $\mathrm{n}=5$, errors are standard error of the mean.

Table 2.1 also gives the average values of oxidation and reduction peak separation of dopamine $\left(\Delta \mathrm{E}_{\mathrm{p}}\right)$ for CNT-grown microelectrodes and CFMEs. The $\Delta \mathrm{E}_{\mathrm{p}}$ values are significantly lower at CNT-Nb (one way-ANOVA Bonferonni post-test, $\mathrm{p}<0.01, \mathrm{n}=5$ ), yielding a peak separation that is $\sim 140 \mathrm{mV}$ and $\sim 80 \mathrm{mV}$ lower than CNT-Ta and CNT-CF microelectrodes, respectively. The smaller $\Delta \mathrm{E}_{\mathrm{p}}$ at CNT-Nb microelectrode might be caused by differing doublelayer capacitances, uncompensated resistance, or ohmic drop. ${ }^{5}$ However, because both the electrolyte and the size of the electrodes are similar, ohmic drop is an unlikely cause. ÁlvarezMartos et al. found that electron transfer was faster through oriented forest-like CNTs than nonoriented, spaghetti-like CNTs. ${ }^{14}$ The CNT-Nb morphology is denser and shorter than CNT-Ta and CNT-CF, and the ends of the tubes are likely to have exposed defects sites for electron transfer. The mass transport per defect would be lower at CNT-Nb, based on the theory of charge transfer at partially blocked surfaces. ${ }^{48}$ The larger number of active sites results in a reduced diffusional flux per active site, which may also be the cause of the smaller $\Delta \mathrm{E}_{\mathrm{p}}$. In addition, there may be restricted mass transfer between the longer CNTs in spaghetti-like CNT grown on Ta and CF. The overall $\Delta \mathrm{E}_{\mathrm{p}}$ at CNT-Nb microelectrodes is larger than CFMEs, which shows that there are likely multiple factors affecting the electron transfer and the rate is also likely depressed by slow transfer through the $\mathrm{Al}_{2} \mathrm{O}_{3}$ buffer layer. 


\subsubsection{Characterization of other metal substrates for CNT growth}

Other metal wires were tested for growing CNTs including molybdenum (Mo), tungsten (W), palladium (Pd), stainless steel (SS), and titanium (Ti). Figure 2.7 shows the SEM images of both bare metals and CNT-grown metals. Larger CNT structures are apparent on W, Pd, SS, and Ti. On Pd, the carbon nanomaterial is larger in diameter and appears to be amorphous carbon, not CNTs. Moreover, CNTs grown on these metals are less dense than the CNTs grown on Nb, Ta, and CF. Background currents at these bare metal wires were approximately 3 to 10 times larger (Fig. 2.8A-E) than the bare $\mathrm{Nb}$ and Ta microelectrodes (Fig. 2.3A-B). None of the bare metals were electrochemically active towards dopamine (Fig. 2.8F-J). After CNT growth, Mo, W, SS, and Ti do not have the typical, characteristic peaks for dopamine even after 160 min of equilibration (Fig. 2.9). Carbon-coated Pd shows electrochemical activity to dopamine (Fig. 2.9H), which is likely due to the amorphous carbon grown on Pd wires. 

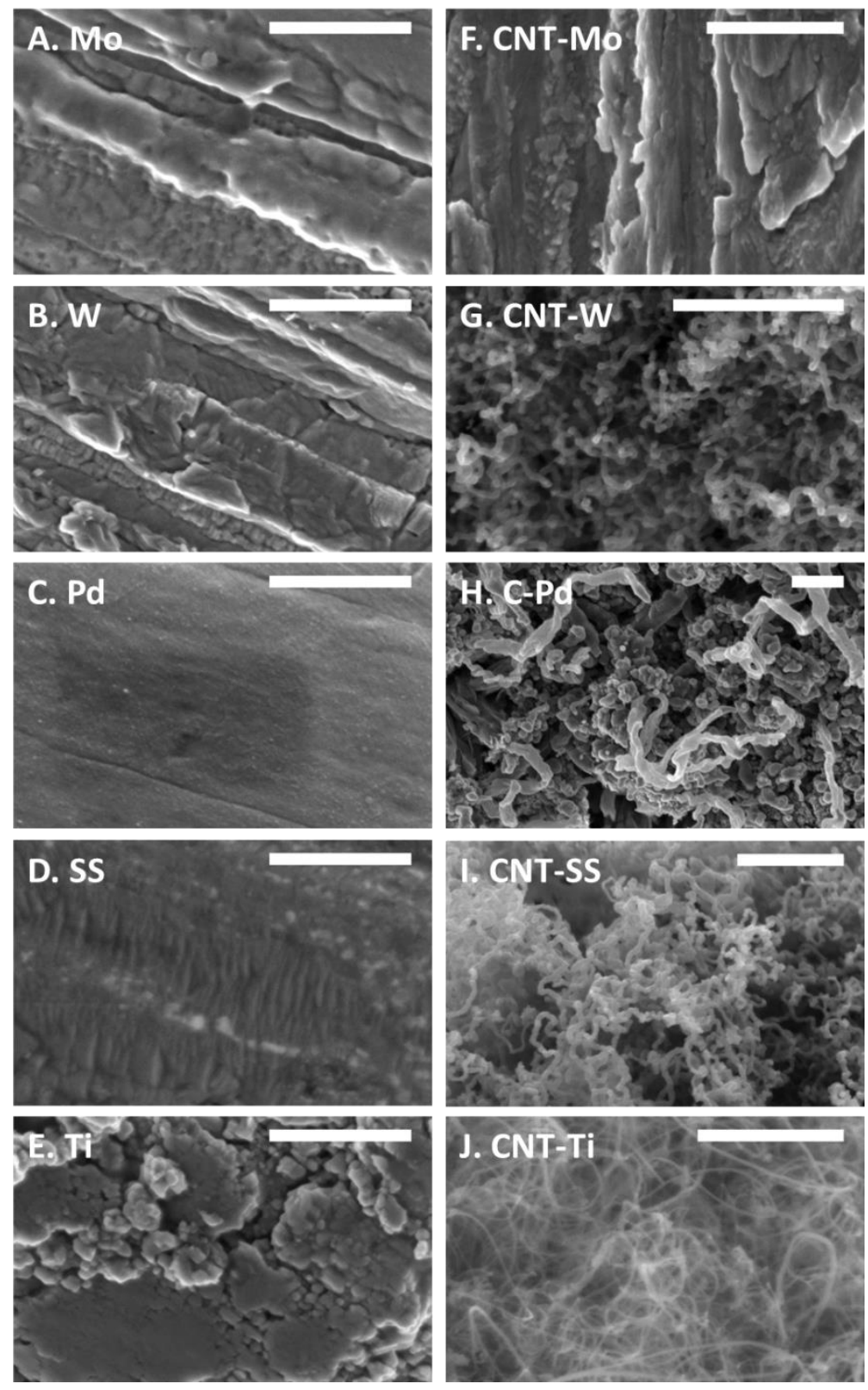

Figure 2.7: SEM images of $(A)$ bare molybdenum, $(B)$ bare tungsten, $(C)$ bare palladium, $(D)$ bare stainless steel, (E) bare titanium, (F) CNT-grown molybdenum, (G) CNT-grown tungsten, (H) carbon-grown palladium, (I) CNT-grown stainless steel, and (J) CNT-grown titanium. Scale bar: $500 \mathrm{~nm}$. 
Background

A. Molybdenum

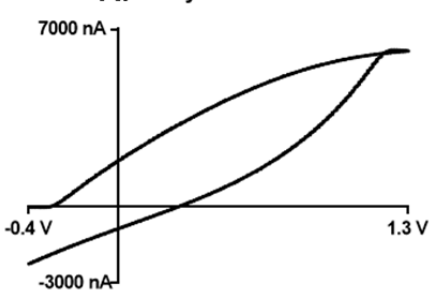

B. Tungsten

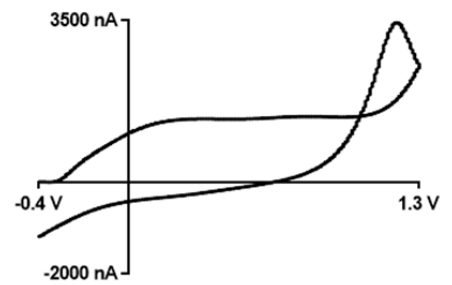

C. Palladium

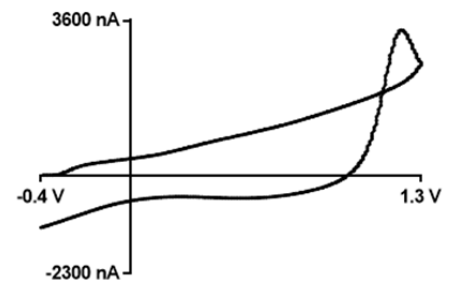

D. Stainless Steel

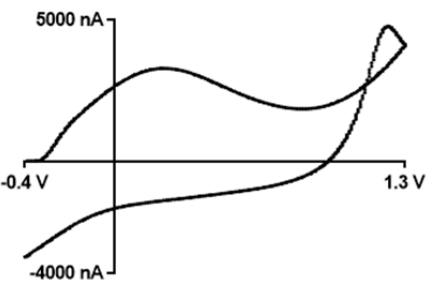

E. Titanium

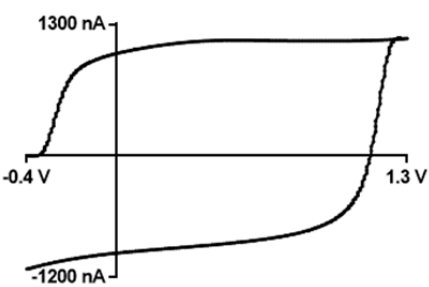

Dopamine

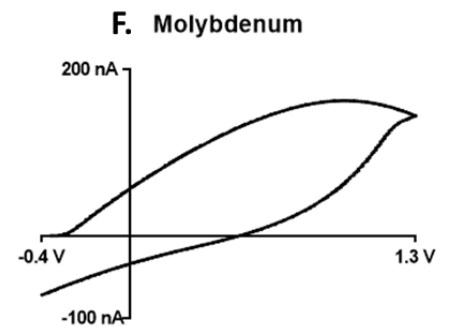

G. Tungsten

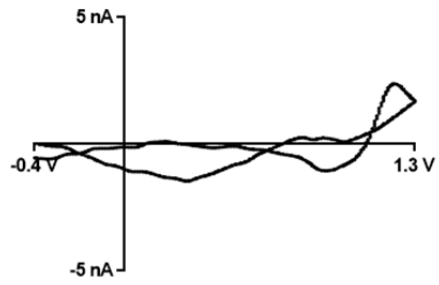

H. Palladium

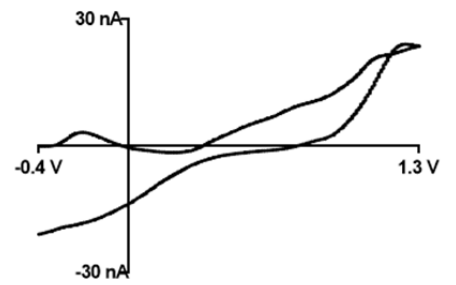

I. Stainless Steel

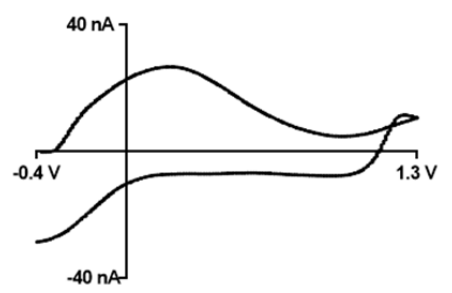

J. Titanium

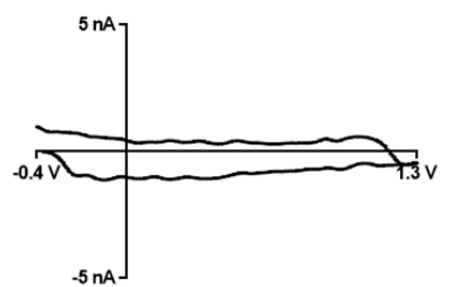

Figure 2.8: Electrochemical response of bare metals. Background current in PBS buffer solution for A. Molybdenum, B. Tungsten, C. Palladium, D. Stainless steel, and E. Titanium microelectrodes, and background subtracted cyclic voltammograms for $1 \mu \mathrm{M}$ dopamine at bare F. Molybdenum, G. Tungsten, H. Palladium, I. Stainless steel, and J. Titanium microelectrodes. 
Background

A. CNT-Mo

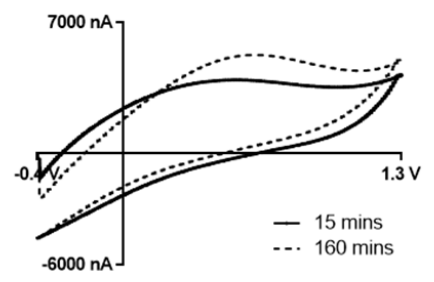

B. CNT-W

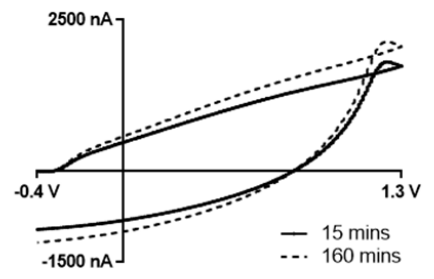

C. CNT-Pd

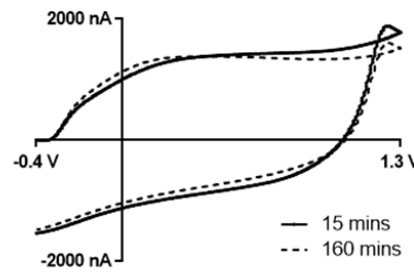

D. CNT-SS

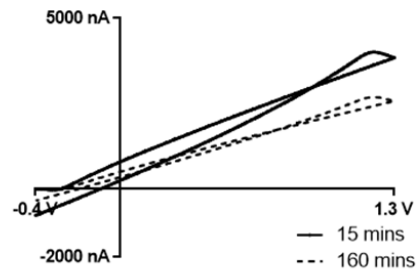

E. CNT-Ti

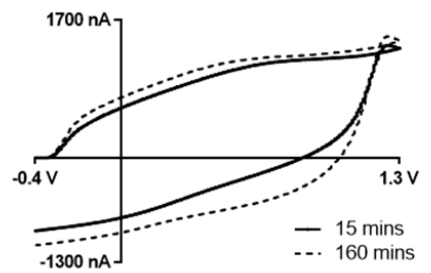

Dopamine

F. CNT-MO

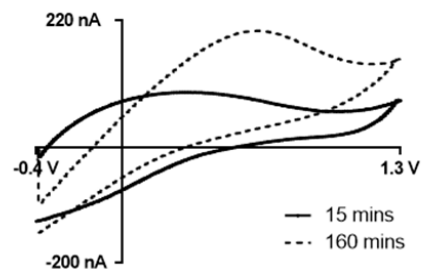

G. CNT-W

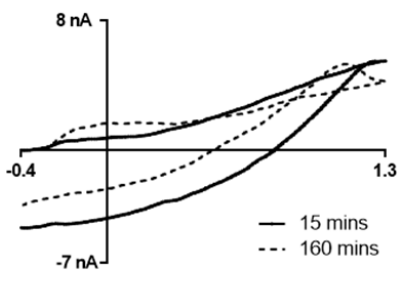

H. CNT-Pd

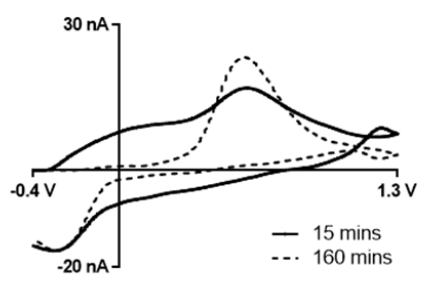

I. CNT-SS

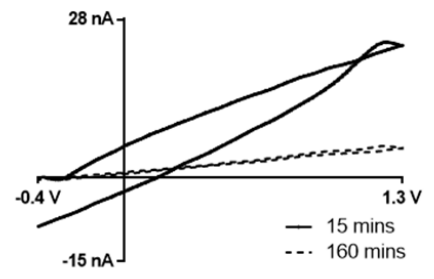

J. CNT-Ti

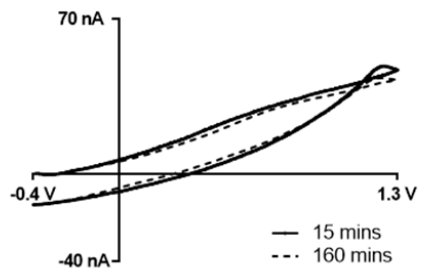

Figure 2.9: Comparison of the electrochemical response at CNT-grown metals microelectrodes. Background current at A. CNT-Molybdenum, B. CNT-Tungsten, C. CNT-Palladium, D. CNTStainless steel, and E. CNT-Titanium microelectrodes, and background-subtracted cyclic voltammogram for $1 \mu \mathrm{M}$ dopamine at F. CNT-Molybdenum, G. CNT-Tungsten, H.CNT-Palladium, I. CNT-Stainless steel, and J. CNT-Titanium microelectrodes. The solid line is the measurements taken after 15 min equilibration and the dashed line is after 160 min equilibration in PBS buffer solution using a waveform of -0.4 to $1.3 \mathrm{~V}$ and back at $400 \mathrm{~V} / \mathrm{s}, 10 \mathrm{~Hz}$. 
Our studies showed that the size of CNTs and the amount of growth depended on the metal substrate. CNT-Nb microelectrodes are preferred for neurotransmitters detection due to the short, dense, and vertically-aligned CNT coating which leads to high current density, low LOD, fast electron transfer rate and short equilibration time. Thus, CNT-Nb microelectrodes were used for in vivo characterization studies.

\subsubsection{Langmuir isotherm modeling}

The redox reaction of dopamine at the surface of carbon based sensors is an adsorption controlled process. ${ }^{49}$ Using a model for FSCV data developed by the Wightman group, ${ }^{50}$ we previously determined that the adsorption/desorption kinetics of dopamine are different for CNT yarn electrodes than CFMEs. ${ }^{51}$ Here, we used a Langmuir adsorption isotherm to model the adsorption and desorption kinetics of dopamine at CNT-grown electrodes. The percent surface coverage is calculated from equation 2.1 and then the coverage vs concentration is fit with the Langmuir isotherm. The anodic peak (Fig. 2.10) and the cathodic peak (Fig. 2.11) give information about dopamine and $\mathrm{DOQ}$ adsorption, respectively. Table 2.2 gives average adsorption rate constants for CNT-Nb, CNT-Ta, and CNT-CF microelectrodes as well as bare CFMEs. The $\beta$ value is used to calculate the Gibbs free energy for dopamine and DOQ adsorption. 
Table 2.2: Average equilibrium constants and adsorption Gibbs free energy for dopamine and DOQ at CNT-grown microelectrodes and CFMEs.

\begin{tabular}{|c|c|c|c|c|c|c|}
\hline Material & $\begin{array}{c}\beta_{D A} \\
\left({ }^{*} 10^{3}\right)\end{array}$ & $\begin{array}{c}\beta_{D O Q} \\
\left({ }^{\star} 10^{3}\right)\end{array}$ & $\beta_{D A} / \beta_{D O Q}$ & $\underset{(\mathrm{KJ} / \mathrm{mol})}{\Delta \mathrm{G}^{\circ}{ }^{\circ}}$ & $\begin{array}{c}\Delta \mathrm{G}^{\circ} \mathrm{DOQ} \\
(\mathrm{KJ} / \mathrm{mol})\end{array}$ & $\begin{array}{l}\Delta G^{\circ}{ }^{D A} / \\
\Delta G^{\circ}{ }^{D O Q}\end{array}$ \\
\hline CNT-Nb & $21 \pm 1$ & $20 \pm 1$ & $1.03 \pm 0.04$ & $-24.1 \pm 0.1$ & $-24.0 \pm 0.1$ & $1.003 \pm 0.004$ \\
\hline CNT-Ta & $23 \pm 3$ & $23 \pm 5$ & $1.05 \pm 0.09$ & $-24.4 \pm 0.3$ & $-24.3 \pm 0.4$ & $1.003 \pm 0.009$ \\
\hline CNT-CF & $37 \pm 4$ & $31 \pm 2$ & $1.10 \pm 0.02$ & $-25.6 \pm 0.2$ & $-25.1 \pm 0.1$ & $1.014 \pm 0.005$ \\
\hline CFME & $39 \pm 1$ & $32 \pm 1$ & $1.23 \pm 0.04$ & $-25.9 \pm 0.1$ & $-25.2 \pm 0.1$ & $1.019 \pm 0.003$ \\
\hline
\end{tabular}

All $\mathrm{n}=5$. Errors are standard error of the mean.

A.

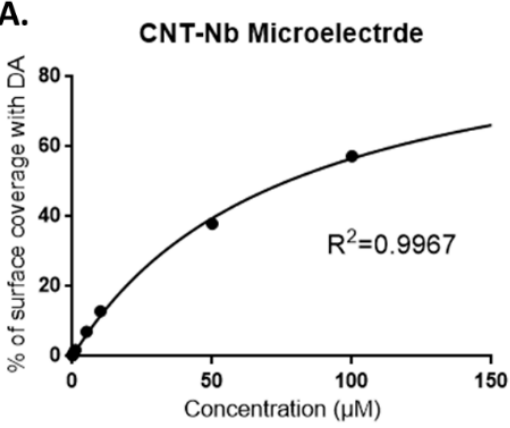

C.

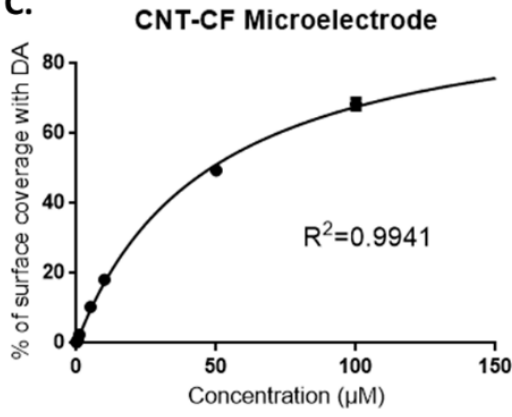

B.

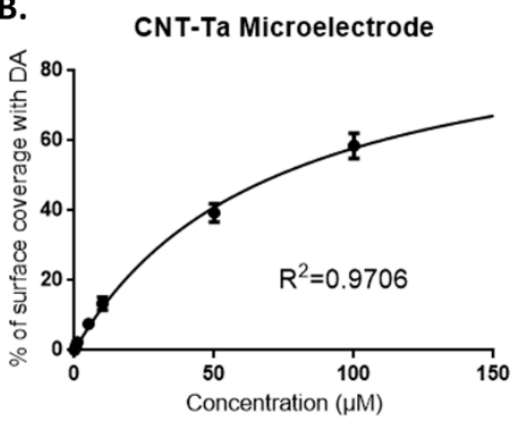

D.

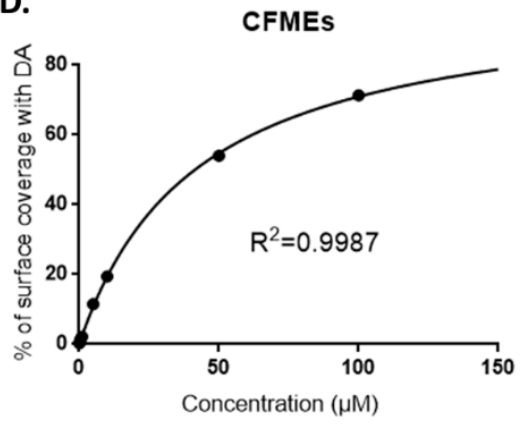

Figure 2.10: Plot of normalized anodic current to corresponding dopamine concentration. The fitting curve is modeled based on Equation 3, where $\mathrm{C}_{\mathrm{DA}}$ is the $\mathrm{X}$ axis and the fractional surface coverage is the $Y$ axis. An equilibrium value, $\beta_{\mathrm{DA}}$, is fit for each curve. $A$. CNT coated $\mathrm{Nb}$ ( $\beta_{\mathrm{DA}}=$ $22,500 \pm 600)$, B. CNT coated Ta $\left(\beta_{D A}=24,600 \pm 3200\right)$, C. CNT coated CF microelectrodes ( $\beta_{D A}$ $=39,700 \pm 3500)$, and D. CFMEs $\left(\beta_{D A}=42,300 \pm 1100\right)(n=5$ per electrode material, error bar is standard error of mean and sometimes is so small as to be less than the size of the point). 
A.

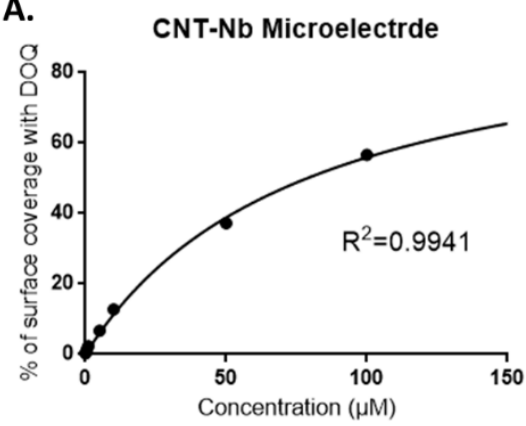

C.

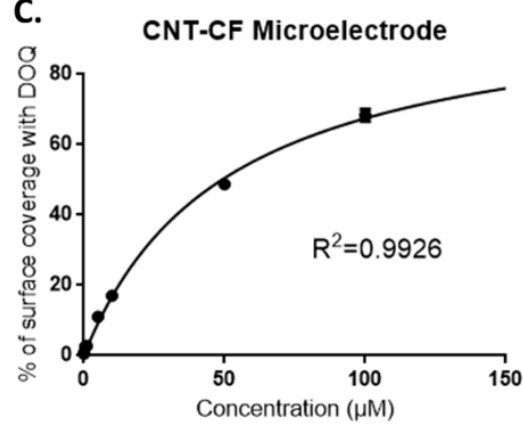

B.

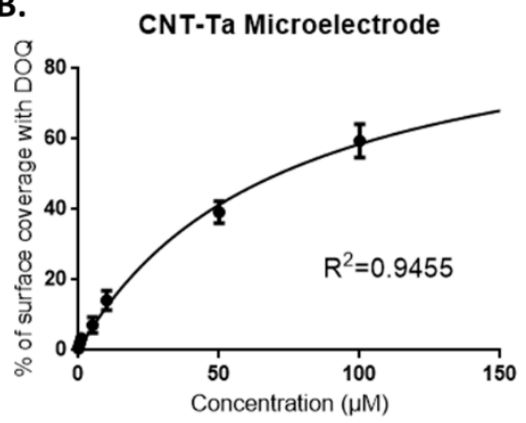

D.

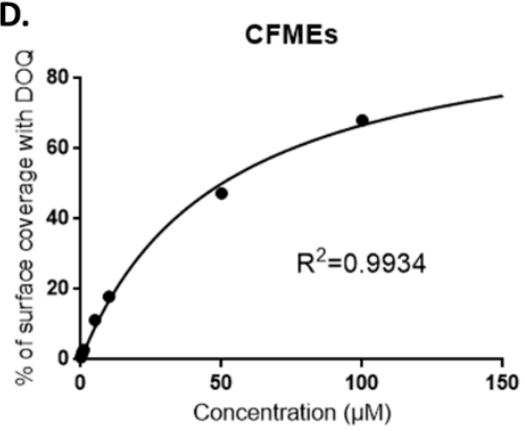

Figure 2.11: Plot of normalized cathodic current to corresponding dopamine concentration. The fitting curve is modeled based on Equation 3, where $C_{D A}$ is the $X$ axis and the fractional surface coverage is the $Y$ axis. An equilibrium value, $\beta_{\mathrm{DOQ}}$, is fit for each curve. A. CNT coated Nb ( $\beta_{\mathrm{DOQ}}$ $=21,900 \pm 900) \mathrm{B}$. CNT coated Ta $\left(\beta_{\mathrm{DOQ}}=25,300 \pm 5600\right) \mathrm{C}$. CNT coated CF microelectrodes $\left(\beta_{\mathrm{DOQ}}=34,000 \pm 1500\right)$ and D. CFMEs $\left(\beta_{\mathrm{DOQ}}=34,600 \pm 1400\right)(n=5$ per electrode material, error bar is standard error of mean).

Both the adsorption equilibrium for dopamine $\left(\beta_{\mathrm{DA}}\right)$ and for $\mathrm{DOQ}\left(\beta_{\mathrm{DOQ}}\right)$ at CNT-Nb and CNT-Ta microelectrodes are smaller than those at CFMEs and CNT-CF microelectrodes. This indicates dopamine and DOQ adsorb stronger to CFMEs and CNT-CFs than to CNT-Nb and CNTTa microelectrodes. However, at the CNT-Ta and CNT-Nb microelectrodes, $\beta_{\mathrm{DA}}$ is similar to $\beta_{\mathrm{DOQ}}$, and the ratio of $\beta_{D A} / \beta_{D O Q}$ is about 1 (Table 2.2). At CFMEs, $\beta_{D A}$ is significantly larger than $\beta_{D O Q}$ (paired t-test, $p<0.005, n=5$ ), and the $\beta_{\mathrm{DA}} / \beta_{\mathrm{DOQ}}$ ratio is larger than $\mathrm{CNT}-\mathrm{Nb}$ microelectrodes (unpaired t-test, $\mathrm{p}<0.05, \mathrm{n}=5$ ). Thus, $\mathrm{DOQ}$ is more likely to re-adsorb from the electrode at CNT-Nb electrodes, leading to a bigger reduction peak and more reversible reaction. The ratio of equilibrium constants for CNT-CF microelectrodes falls in between that of CFMEs and CNT-grown wires. The overall equilibrium is likely a convolution of the equilibrium at CNT-coated parts of the 
electrode and bare CFME, which is also partially exposed to solution. These data agreed with previous modeling of CNT yarn electrodes, which showed differences in adsorption for dopamine and DOQ compared to CFMEs. ${ }^{51}$

\subsubsection{FSCV of other neurochemicals}

We tested the electrochemical performance of CNT-Nb microelectrodes towards the detection of other neurochemicals including ascorbic acid (AA), DOPAC (3,4Dihydroxyphenylacetic acid), serotonin, adenosine, and histamine. Since the detection of adenosine and histamine requires scanning to higher potentials, we used a waveform of $-0.4 \mathrm{~V}$ to $1.45 \mathrm{~V}$ at $400 \mathrm{~V} / \mathrm{s}^{52}$ Figure 2.12 shows the example $\mathrm{CVs}$ for each neurochemical (black solid line) compared to dopamine (red dashed line) at the same CNT-Nb electrode. The bar graphs compare the ratio of oxidation currents of the different neurotransmitters to dopamine at CNT-Nb microelectrodes and CFMEs. Ascorbic acid is an anionic antioxidant present in high concentrations in the brain, ${ }^{53}$ with a broad oxidation peak near the potential for dopamine detection (Fig. 2.12A). The ratios of oxidative current for $200 \mu \mathrm{M}$ AA to $1 \mu \mathrm{M}$ dopamine at CNT$\mathrm{Nb}$ microelectrodes are significantly smaller than those at CFMEs (Fig 2.12B, paired t-test, $\mathrm{p}<$ 0.0001, $n=5$ ), indicating CNT-Nb microelectrodes have better selectivity towards dopamine over AA than CFMEs. Since $\mathrm{AA}$ is an anion at physiological $\mathrm{pH},{ }^{54}$ the abundant oxygen-containing functional groups on CNTs surface might repel AA and further increase the selectivity to cationic dopamine. DOPAC is a dopamine metabolite ${ }^{55}$ and has a similar oxidation potential to dopamine (Fig 2.12C). Although there is no significant difference in selectivity to dopamine over DOPAC at CNT-Nb microelectrodes (Fig 2.12D, paired t-test, $p=0.1454, n=5$ ), the reduction potential of DOPAC is significantly more negative $(-0.28 \mathrm{~V} \pm 0.01 \mathrm{~V}$ for DOPAC compared to $-0.22 \mathrm{~V} \pm 0.01$ $\mathrm{V}$ for dopamine, paired t-test, $\mathrm{p}<0.001, \mathrm{n}=5$ ), similar to previous CNT electrode studies. ${ }^{9}$ Serotonin is a cationic, indolamine neurotransmitter. ${ }^{56}$ The ratio of currents for serotonin to dopamine is similar for CNT-Nb microelectrodes and CFMEs (Fig. 2.12F, paired t-test, $p=0.3008$, 
$n=5)$. The oxidation peak for serotonin is similar to dopamine as well, but the reduction peak is shifted by $200 \mathrm{mV}$ (Fig. 2.12E, paired t-test, $p<0.0001, n=5$ ), which can be used to discriminate serotonin from dopamine. Adenosine is an important neuroprotective modulator in the brain that regulates neurotransmission and blood flow. ${ }^{52}$ Adenosine is identified by its two oxidation peaks in the CV (a primary oxidation peak at $1.4 \mathrm{~V}$ and a secondary peak at $1.0 \mathrm{~V}$, Fig. $2.12 \mathrm{G}$ ). ${ }^{52}$ The selectivity to adenosine compared to dopamine at CNT-Nb microelectrodes is similar to CFMEs (Fig. 2.12H, paired t-test, $\mathrm{p}=0.7476, \mathrm{n}=5$ ). Histamine is a neurotransmitter that regulates sleep. ${ }^{57}$ CNT-Nb electrodes have an oxidation peak near the switching potential (Fig. 2.12I) and show significantly higher histamine to dopamine current ratios than CFMEs (Fig 2.12J, paired t-test, $\mathrm{p}$ $<0.05, \mathrm{n}=5$ ), which might be due to the better antifouling properties of the CNT surface towards histamine than CFMEs. ${ }^{58}$ Overall, CNT-Nb microelectrodes are useful for detecting a variety of neurochemicals. 


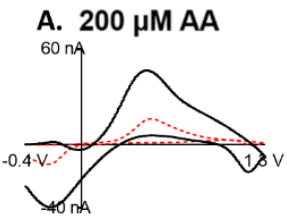

B. $200 \mu \mathrm{M}$ AA

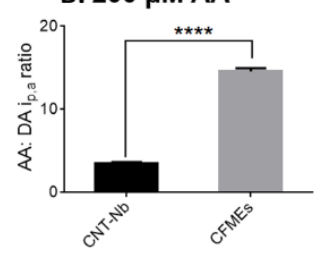

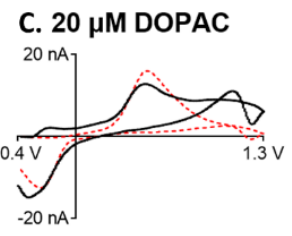

D. $20 \mu \mathrm{M}$ DOPAC

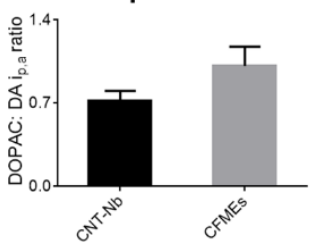

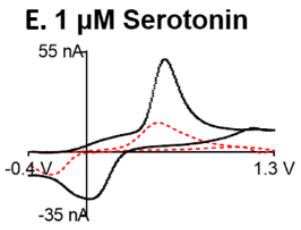

F. $1 \mu \mathrm{M}$ Serotonin

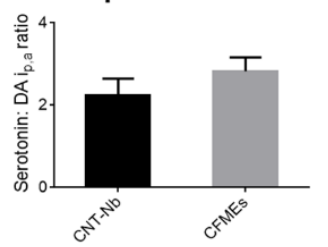

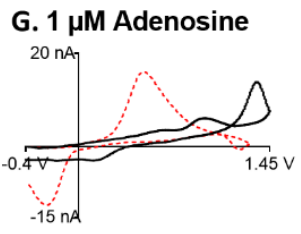

H. $1 \mu \mathrm{M}$ Adenosine

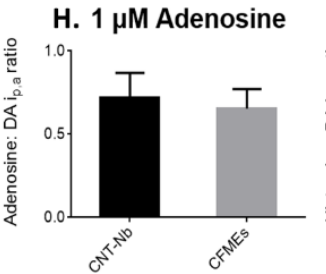

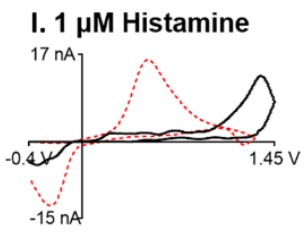

J. $1 \mu \mathrm{M}$ Histamine

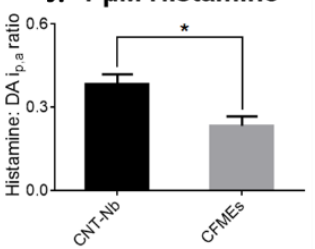

Figure 2.12: Detection of other neurochemicals at CNT-Nb microelectrodes. CVs of A. $200 \mu \mathrm{M}$ AA, C. $20 \mu \mathrm{M}$ DOPAC, E. $1 \mu \mathrm{M}$ serotonin, G. $1 \mu \mathrm{M}$ adenosine, and I. $1 \mu \mathrm{M}$ histamine in PBS buffer. Red dashed line is CV of $1 \mu \mathrm{M}$ dopamine obtained from the same CNT-Nb electrode. For AA, DOPAC and serotonin the electrode was scanned to $1.3 \mathrm{~V}$, for adenosine and histamine, the electrode was scanned to $1.45 \mathrm{~V}$. Column plot shows the ratio of oxidation current for B. $200 \mu \mathrm{M}$ AA, D. $20 \mu \mathrm{M}$ DOPAC, F. $1 \mu \mathrm{M}$ serotonin, H. $1 \mu \mathrm{M}$ adenosine, and J. $1 \mu \mathrm{M}$ histamine compared to the corresponding oxidation current of dopamine at CNT-Nb microelectrode (black, $n=5$ ) and CFMEs (gray, $n=5$ ). The oxidation current ratios at CNT-Nb microelectrodes are significantly different than CFMEs for the measurement of ascorbic acid (paired t-test, $p<0.0001$ ) and histamine (paired t-test, $\mathrm{p}<0.05$ ).

\subsubsection{In vivo detection of dopamine at CNT-Nb microelectrodes}

To determine the applicability of the CNT-Nb microelectrode as a novel in vivo sensor, stimulated dopamine release was measured in anesthetized, male Sprague-Dawley rats. Stimulation pulse trains were applied ( $300 \mu \mathrm{A}, 12-120$ pulses, $60 \mathrm{~Hz})$ to the dopamine cell bodies and the dopamine response recorded in the caudate-putamen near the terminals. Figure $2.13 \mathrm{~A}$ and B show example CVs and current versus time plots of dopamine detection at a CNT-Nb microelectrode with different stimulation pulses. Current increased as dopamine was released during the stimulation and decreased after the stimulation due to uptake..$^{53}$ Figure $2.13 \mathrm{C}$ gives the average dopamine concentration evoked in vivo; released dopamine is still detectable with as low as 12 stimulation pulses. The current density of the stimulated dopamine at CNT-Nb microelectrode in vivo $\left(0.15 \pm 0.02 \mathrm{pA} /\left(\mathrm{nM} \cdot \mu \mathrm{m}^{2}\right)\right)$ is slightly lower than that in vitro $(0.20 \pm 0.02$ $\mathrm{pA} /\left(\mathrm{nM} \cdot \mu^{2}\right)$ ) but not significantly different (unpaired t-test, $\left.\mathrm{p}>0.05\right)$, which indicates CNT-Nb microelectrodes maintained relatively high sensitivity for in vivo dopamine detection. The CV of 
dopamine in vivo has a larger $\Delta \mathrm{E}_{\mathrm{p}}$ than that in vitro, likely due to adsorption of lipids, proteins, and peptides present in the extracellular fluid that slow electron transfer. ${ }^{59,60}$ While the larger $\Delta \mathrm{E}_{\mathrm{p}}$ is not ideal, the sensitivity is maintained and the CV could be matched to in vivo spontaneous release. However, future studies could focus on in vivo studies of protein fouling and adopt strategies that have been implemented for gold ${ }^{61}$ and carbon-fiber microelectrodes ${ }^{62}$ to tune the surface adsorption of dopamine.
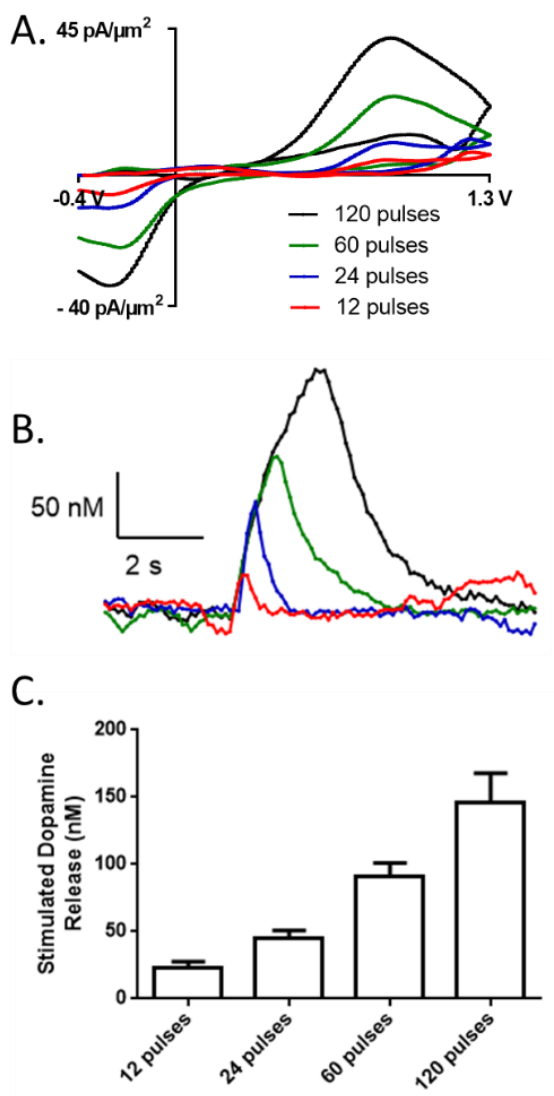

Figure 2.13: Dopamine detection in vivo at CNT-Nb microelectrodes. A. Example CVs depicting stimulated dopamine release detected from a CNT-Nb microelectrode placed in the caudate putamen with a stimulation pulse train of $120,60,24$, and 12 pulses at $60 \mathrm{~Hz}$, respectively. B. The associated concentration vs. time plot. C. Averaged dopamine concentration at different pulses detected at CNT-Nb microelectrodes $(n=4)$. The electrode was scanned from -0.4 to 1.3 $\mathrm{V}$ and back at $400 \mathrm{~V} / \mathrm{s}$ at $10 \mathrm{~Hz}$. 


\subsection{Conclusions}

In summary, we successfully grew CNTs on Nb and used CNTs on cylindrical wires as microelectrodes for the first time. Small wire microelectrodes should minimize tissue damage and improve spatial resolution, which are needed for in vivo applications. This work is the first to compare CNT growth on various metal wires as well as carbon fibers and the comparison is useful in choosing appropriate substrates for future CNT studies. CNT forest-grown on Nb wires are shorter, denser, and more aligned than CNTs grown on other substrates, which led to enhanced current density and better LOD for dopamine. In addition, CNT-Nb microelectrodes are stable over four hours of continuous measurement and are able to measure stimulated dopamine release in anesthetized rats. CNT-Nb microelectrodes have the potential applications for the detection of neurotransmitters in vivo or metal electrode arrays in electrophysiology studies. 


\subsection{References}

(1) Alwarappan, S.; Liu, G.; Li, C.-Z. Simultaneous Detection of Dopamine, Ascorbic Acid, and Uric Acid at Electrochemically Pretreated Carbon Nanotube Biosensors. Nanomedicine 2010, 6 (1), 52-57.

(2) Tsierkezos, N. G.; Ritter, U. Oxidation of Dopamine on Multi-Walled Carbon Nanotubes. J. Solid State Electrochem. 2012, 16 (6), 2217-2226.

(3) Salinas-Torres, D.; Huerta, F.; Montilla, F.; Morallón, E. Study on Electroactive and Electrocatalytic Surfaces of Single Walled Carbon Nanotube-Modified Electrodes. Electrochim. Acta 2011, 56 (5), 2464-2470.

(4) Habibi, B.; Jahanbakhshi, M.; Pournaghi-Azar, M. H. Simultaneous Determination of Acetaminophen and Dopamine Using SWCNT Modified Carbon-ceramic Electrode by Differential Pulse Voltammetry. Electrochim. Acta 2011, 56 (7), 2888-2894.

(5) Zestos, A. G.; Jacobs, C. B.; Trikantzopoulos, E.; Ross, A. E.; Venton, B. J. Polyethylenimine Carbon Nanotube Fiber Electrodes for Enhanced Detection of Neurotransmitters. Anal. Chem. 2014, 86, 8568-8575.

(6) Swamy, B. E. K.; Venton, B. J. Carbon Nanotube-Modified Microelectrodes for Simultaneous Detection of Dopamine and Serotonin in Vivo. Analyst 2007, 132 (9), 876884.

(7) Jacobs, C. B.; Vickrey, T. L.; Venton, B. J. Functional Groups Modulate the Sensitivity and Electron Transfer Kinetics of Neurochemicals at Carbon Nanotube Modified Microelectrodes. Analyst 2011, 136 (17), 3557.

(8) Zestos, A. G.; Nguyen, M. D.; Poe, B. L.; Jacobs, C. B.; Venton, B. J. Epoxy Insulated Carbon Fiber and Carbon Nanotube Fiber Microelectrodes. Sensors Actuators $B$ Chem. 2013, 182 (2013), 652-658.

(9) Peairs, M. J.; Ross, A. E.; Venton, B. J. Comparison of Nafion- and Overoxidized Polypyrrole-Carbon Nanotube Electrodes for Neurotransmitter Detection. Anal. Methods 2011, 3 (10), 2379.

(10) McCreery, R. L. Advanced Carbon Electrode Materials for Molecular Electrochemistry. Chem. Rev. 2008, 108 (7), 2646-2687.

(11) Jacobs, C. B.; Peairs, M. J.; Venton, B. J. Review: Carbon Nanotube Based Electrochemical Sensors for Biomolecules. Anal. Chim. Acta 2010, 662 (2), 105-127.

(12) Liu, J. Fullerene Pipes. Science (80-. ). 1998, 280 (5367), 1253-1256.

(13) Yang, C.; Denno, M. E.; Pyakurel, P.; Venton, B. J. Recent Trends in Carbon NanomaterialBased Electrochemical Sensors for Biomolecules: A Review. Anal. Chim. Acta 2015, 887, 17-37.

(14) Álvarez-Martos, I.; Fernández-Gavela, A.; Rodríguez-García, J.; Campos-Alfaraz, N.; García-Delgado, a. B.; Gómez-Plaza, D.; Costa-García, A.; Fernández-Abedul, M. T. Electrochemical Properties of Spaghetti and Forest like Carbon Nanotubes Grown on Glass Substrates. Sensors Actuators B Chem. 2014, 192 (2014), 253-260.

(15) Xiao, N.; Venton, B. J. Rapid, Sensitive Detection of Neurotransmitters at Microelectrodes Modified with Self-Assembled SWCNT Forests. Anal. Chem. 2012, 84 (18), 7816-7822. 
(16) Xiang, L.; Yu, P.; Hao, J.; Zhang, M.; Zhu, L.; Dai, L.; Mao, L. Vertically Aligned Carbon Nanotube-Sheathed Carbon Fibers as Pristine Microelectrodes for Selective Monitoring of Ascorbate in Vivo. Anal. Chem. 2014, 86 (8), 3909-3914.

(17) Zachek, M. K.; Hermans, A.; Wightman, R. M.; McCarty, G. S. Electrochemical Dopamine Detection: Comparing Gold and Carbon Fiber Microelectrodes Using Background Subtracted Fast Scan Cyclic Voltammetry. J. Electroanal. Chem. 2008, 614 (1-2), 113120.

(18) Ciszewski, A.; Milczarek, G. Polyeugenol-Modified Platinum Electrode for Selective Detection of Dopamine in the Presence of Ascorbic Acid. Anal. Chem. 1999, 71 (5), 10551061.

(19) Swamy, B. E. K.; Venton, B. J. Carbon Nanotube-Modified Microelectrodes for Simultaneous Detection of Dopamine and Serotonin in Vivo. Analyst 2007, 132 (9), $876-$ 884.

(20) Delmas, M.; Pinault, M.; Patel, S.; Porterat, D.; Reynaud, C.; Mayne-L'Hermite, M. Growth of Long and Aligned Multi-Walled Carbon Nanotubes on Carbon and Metal Substrates. Nanotechnology 2012, 23 (10), 105604.

(21) Atthipalli, G.; Epur, R.; Kumta, P. N.; Yang, M.; Lee, J.-K.; Gray, J. L. Nickel CatalystAssisted Vertical Growth of Dense Carbon Nanotube Forests on Bulk Copper. J. Phys. Chem. C 2011, 115 (9), 3534-3538.

(22) Lepró, X.; Lima, M. D.; Baughman, R. H. Spinnable Carbon Nanotube Forests Grown on Thin, Flexible Metallic Substrates. Carbon N. Y. 2010, 48 (12), 3621-3627.

(23) Gao, L.; Peng, A.; Wang, Z. Y.; Zhang, H.; Shi, Z.; Gu, Z.; Cao, G.; Ding, B. Growth of Aligned Carbon Nanotube Arrays on Metallic Substrate and Its Application to Supercapacitors. Solid State Commun. 2008, 146 (9-10), 380-383.

(24) Hiraoka, T.; Yamada, T.; Hata, K.; Futaba, D. N.; Kurachi, H.; Uemura, S.; Yumura, M.; lijima, S. Synthesis of Single- and Double-Walled Carbon Nanotube Forests on Conducting Metal Foils. J. Am. Chem. Soc. 2006, 128 (41), 13338-13339.

(25) Bayer, B. C.; Hofmann, S.; Castellarin-Cudia, C.; Blume, R.; Baehtz, C.; Esconjauregui, S.; Wirth, C. T.; Oliver, R. A.; Ducati, C.; Knop-Gericke, A.; et al. Support-Catalyst-Gas Interactions during Carbon Nanotube Growth on Metallic Ta Films. J. Phys. Chem. C 2011, 115 (11), 4359-4369.

(26) Zestos, A. G.; Yang, C.; Jacobs, C. B.; Hensley, D.; Venton, B. J. Carbon Nanospikes Grown on Metal Wires as Microelectrode Sensors for Dopamine. Analyst 2015, 140 (21), 7283-7292.

(27) Kozai, T. D. Y.; Jaquins-Gerstl, A. S.; Vazquez, A. L.; Michael, A. C.; Cui, X. T. Brain Tissue Responses to Neural Implants Impact Signal Sensitivity and Intervention Strategies. ACS Chem. Neurosci. 2015, 6 (1), 48-67.

(28) Tran, K. Y.; Heinrichs, B.; Colomer, J.-F.; Pirard, J.-P.; Lambert, S. Carbon Nanotubes Synthesis by the Ethylene Chemical Catalytic Vapour Deposition (CCVD) Process on Fe, Co, and Fe-Co/Al2O3 Sol-gel Catalysts. Appl. Catal. A Gen. 2007, 318 (2007), 63-69.

(29) Strand, A. M.; Venton, B. J. Flame Etching Enhances the Sensitivity of Carbon-Fiber Microelectrodes. Anal. Chem. 2008, 80 (10), 3708-3715.

(30) Graham, D. The Characterization of Physical Adsorption Systems. I. The Equilibrium 
Function and Standard Free Energy of Adsorption. J. Phys. Chem. 1953, 57 (7), 665-669.

(31) Liu, Y. Is the Free Energy Change of Adsorption Correctly Calculated? J. Chem. Eng. Data 2009, 54 (7), 1981-1985.

(32) Archer, D. G.; Wang, P. The Dielectric Constant of Water and Debye-Hückel Limiting Law Slopes. J. Phys. Chem. Ref. Data 1990, 19 (2), 371.

(33) Mortimer, R. G. Physical Chemistry, Third Edit.; Academic Press: Memphis, TN, USA, 2008.

(34) Holloway, A. F.; Toghill, K.; Wildgoose, G. G.; Compton, R. G.; Ward, M. a H.; Tobias, G.; Llewellyn, S. a; Ballesteros, B.; Green, M. L. H.; Crossley, A. Electrochemical Opening of Single-Walled Carbon Nanotubes Filled with Metal Halides and with Closed Ends. J. Phys. Chem. C 2008, 112 (28), 10389-10397.

(35) Magrez, A.; Seo, J.; Smajda, R.; Mionić, M.; Forró, L. Catalytic CVD Synthesis of Carbon Nanotubes: Towards High Yield and Low Temperature Growth. Materials (Basel). 2010, 4871-4891.

(36) Matthews, K. D.; Lemaitre, M. G.; Kim, T.; Chen, H.; Shim, M.; Zuo, J.-M. Growth Modes of Carbon Nanotubes on Metal Substrates. J. Appl. Phys. 2006, 100 (4), 44309.

(37) Delzeit, L.; Nguyen, C. V.; Chen, B.; Stevens, R.; Cassell, A.; Han, J.; Meyyappan, M. Multiwalled Carbon Nanotubes by Chemical Vapor Deposition Using Multilayered Metal Catalysts. J. Phys. Chem. B 2002, 106, 5629-5635.

(38) Parthangal, P. M.; Cavicchi, R. E.; Zachariah, M. R. A Generic Process of Growing Aligned Carbon Nanotube Arrays on Metals and Metal Alloys. Nanotechnology 2007, 18 (18), 185605.

(39) Burt, D. P.; Whyte, W. M.; Weaver, J. M. R.; Glidle, A.; Edgeworth, J. P.; Macpherson, J. V.; Dobson, P. S. Effects of Metal Underlayer Grain Size on Carbon Nanotube Growth. J. Phys. Chem. C 2009, 113 (34), 15133-15139.

(40) Raney, J. R.; Misra, A.; Daraio, C. Tailoring the Microstructure and Mechanical Properties of Arrays of Aligned Multiwall Carbon Nanotubes by Utilizing Different Hydrogen Concentrations during Synthesis. Carbon N. Y. 2011, 49 (11), 3631-3638.

(41) Uehara, I.; Sakai, T.; Ishikawa, H.; Takenaka, H. Corrosion Behavior of Tantalum and Niobium in Hydrobromic Acid Solutions (II) on Passive Films and Hydrogen Absorption. Corrosion 1989, 45 (7), 548-553.

(42) Lewis, F. a. Solubility of Hydrogen in Metals. Pure Appl. Chem. 1990, 62 (11), 2091-2096.

(43) Davenport, J.; Dienes, G.; Johnson, R. Surface Effects on the Kinetics of Hydrogen Absorption by Metals. Phys. Rev. B 1982, 25 (4).

(44) Filik, J.; May, P. W.; Pearce, S. R. J.; Wild, R. K.; Hallam, K. R. XPS and Laser Raman Analysis of Hydrogenated Amorphous Carbon Films. Diam. Relat. Mater. 2003, 12 (3-7), 974-978.

(45) Feng, X.; Liu, K.; Xie, X.; Zhou, R.; Zhang, L.; Li, Q.; Fan, S.; Jiang, K. Thermal Analysis Study of the Growth Kinetics of Carbon Nanotubes and Epitaxial Graphene Layers on Them. J. Phys. Chem. C 2009, 113 (22), 9623-9631.

(46) Takmakov, P.; Zachek, M. K.; Keithley, R. B.; Walsh, P. L.; Donley, C.; McCarty, G. S.; Wightman, R. M. Carbon Microelectrodes with a Renewable Surface. Anal. Chem. 2010, 82 (5), 2020-2028. 
(47) Miller, T. S.; Macpherson, J. V; Unwin, P. R. Electrochemical Activation of Pristine Single Walled Carbon Nanotubes: Impact on Oxygen Reduction and Other Surface Sensitive Redox Processes. Phys. Chem. Chem. Phys. 2014, 16 (21), 9966.

(48) Amatore, C.; Savéant, J. M.; Tessier, D. Charge Transfer at Partially Blocked Surfaces. J. Electroanal. Chem. Interfacial Electrochem. 1983, 147 (1-2), 39-51.

(49) Atcherley, C. W.; Laude, N. D.; Parent, K. L.; Heien, M. L. Fast-Scan Controlled-Adsorption Voltammetry for the Quantification of Absolute Concentrations and Adsorption Dynamics. Langmuir 2013, 29 (48), 14885-14892.

(50) Bath, B. D.; Michael, D. J.; Trafton, B. J.; Joseph, J. D.; Runnels, P. L.; Wightman, R. M. Subsecond Adsorption and Desorption of Dopamine at Carbon-Fiber Microelectrodes. Anal. Chem. 2000, 72 (24), 5994-6002.

(51) Jacobs, C. B.; Ivanov, I. N.; Nguyen, M. D.; Zestos, A. G.; Venton, B. J. High Temporal Resolution Measurements of Dopamine with Carbon Nanotube Yarn Microelectrodes. Anal. Chem. 2014, 86 (12), 5721-5727.

(52) Nguyen, M. D.; Lee, S. T.; Ross, A. E.; Ryals, M.; Choudhry, V. I.; Venton, B. J. Characterization of Spontaneous, Transient Adenosine Release in the Caudate-Putamen and Prefrontal Cortex. PLoS One 2014, 9 (1), e87165.

(53) Robinson, D. L.; Venton, B. J.; Heien, M. L. a V; Wightman, R. M. Detecting Subsecond Dopamine Release with Fast-Scan Cyclic Voltammetry in Vivo. Clin. Chem. 2003, 49 (10), 1763-1773.

(54) Pisoschi, A. M.; Pop, A.; Serban, A. I.; Fafaneata, C. Electrochemical Methods for Ascorbic Acid Determination. Electrochim. Acta 2014, 121, 443-460.

(55) Huffman, M. L.; Venton, B. J. Electrochemical Properties of Different Carbon-Fiber Microelectrodes Using Fast-Scan Cyclic Voltammetry. Electroanalysis 2008, 20 (22), 2422-2428.

(56) Baur, J. E.; Kristensen, E. W.; May, L. J.; Wiedemann, D. J.; Wightman, R. M. Fast-Scan Voltammetry of Biogenic Amines. Anal. Chem. 1988, 60 (13), 1268-1272.

(57) Robinson, D. L.; Hermans, A.; Seipel, A. T.; Wightman, R. M. Monitoring Rapid Chemical Communication in the Brain. Chem. Rev. 2008, 108 (7), 2554-2584.

(58) Ross, A. E.; Venton, B. J. Nafion-CNT Coated Carbon-Fiber Microelectrodes for Enhanced Detection of Adenosine. Analyst 2012, 137 (13), 3045-3051.

(59) Chandra, S.; Miller, A. D.; Bendavid, A.; Martin, P. J.; Wong, D. K. Y. Minimizing Fouling at Hydrogenated Conical-Tip Carbon Electrodes during Dopamine Detection in Vivo. Anal. Chem. 2014, 86 (5), 2443-2450.

(60) Park, J.; Show, Y.; Quaiserova, V.; Galligan, J. J.; Fink, G. D.; Swain, G. M. Diamond Microelectrodes for Use in Biological Environments. J. Electroanal. Chem. 2005, 583 (1), 56-68.

(61) Chen, C.-H.; Luo, S.-C. Tuning Surface Charge and Morphology for the Efficient Detection of Dopamine under the Interferences of Uric Acid, Ascorbic Acid, and Protein Adsorption. ACS Appl. Mater. Interfaces 2015, 7 (39), 21931-21938.

(62) Singh, Y. S.; Sawarynski, L. E.; Dabiri, P. D.; Choi, W. R.; Andrews, A. M. Head-to-Head Comparisons of Carbon Fiber Microelectrode Coatings for Sensitive and Selective 
Neurotransmitter Detection by Voltammetry. Anal. Chem. 2011, 83 (17), 6658-6666. 


\section{Chapter 3: Laser Treated Carbon Nanotube Yarn}

Microelectrodes for Rapid and Sensitive Detection of

Dopamine in Vivo 


\section{Abstract}

Carbon nanotube yarn microelectrodes (CNTYMEs) exhibit rapid and selective detection of dopamine with fast-scan cyclic voltammetry (FSCV); however, the sensitivity limits their application in vivo. In this study, we introduce laser treatment as a simple, reliable, and efficient approach to improve the sensitivity of CNTYMEs by three fold while maintaining high temporal resolution. The effect of laser treatment on the microelectrode surface was characterized by scanning electron microscopy, Raman spectroscopy, energy dispersion spectroscopy, and laser confocal microscopy. Laser treatment increases the surface area and oxygen containing functional groups on the surface, which provides more adsorption sites for dopamine than at unmodified CNTYMEs. Moreover, similar to unmodified CNTYMEs, the dopamine signal at laser treated CNTYMEs is not dependent on scan repetition frequency, unlike the current at carbon fiber microelectrodes (CFMEs) which decreases with increasing scan repetition frequency. This frequency independence is caused by the significantly larger surface roughness which would trap dopamine-o-quinone and amplify the dopamine signal. CNTYMEs were applied as an in vivo sensor with FSCV for the first time and laser treated CNTYMEs maintained high dopamine sensitivity compared to CFMEs with an increased scan repetition frequency of $50 \mathrm{~Hz}$, which is five-fold faster than the conventional frequency. CNTYMEs with laser treatment are advantageous because of their easy fabrication, high reproducibility, fast electron transfer kinetics, high sensitivity, and rapid in vivo measurement of dopamine and could be a potential alternative to CFMEs in the future. This paper was published in ACS Sensor (ACS Sensors, 2016, 1 (5), 508$515)$. 


\subsection{Introduction}

Carbon nanotubes (CNTs) are a promising microelectrode material because of their large surface area, abundant elecroactive sites for biomolecules, and fast electron transfer kinetics. ${ }^{1-4}$ CNT-based microelectrodes have been fabricated by modifying a carbon fiber or metal substrate with CNTs ${ }^{5-8}$ or spinning CNTs into continuous fibers ${ }^{9-11}$. The commonly used methods to modify CNTs on carbon fiber or metal microelectrodes include dip coating ${ }^{12}$, covalently bonding CNTs ${ }^{7}$, and directly growing CNTs on substrates ${ }^{5,8}$. In comparison, directly producing CNT fibers is more reproducible ${ }^{13}$ and CNT fibers can be directly fabricated into electrodes in a manner similar to carbon fiber. ${ }^{10}$ Solution based wet-spinning is one of the major routes for CNT fiber fabrication; ${ }^{13}$ macroscopic CNT fibers are spun into a polymer bath, such as poly(vinyl alcohol) (PVA) ${ }^{14}$ or poly(ethylene)imine ${ }^{10}$. The polymer molecules act as binders for CNTs to enhance the intertube interactions; however, the electrical conductivity of the fibers is reduced due to the presence of insulating substances on the path for electron transfer. ${ }^{13,15}$ Continuous CNT yarns, generated by drawing one end of the vertically aligned CNT arrays, are favorable because of better CNT alignment and improved conductivity. ${ }^{16,17}$ Jacobs et al. and Schmidt et al. reported the use CNT yarn as microelectrodes material for neurotransmitter detection. ${ }^{9,11}$ Compared to conventionally used carbon-fiber microelectrode (CFME), CNT yarn microelectrodes (CNTYMEs) exhibit fast electron transfer kinetics and high detection sensitivity.

Neurotransmitter detection in the intact brain requires high temporal resolution, chemical identification, and low limits of detection (LOD). Fast-scan cyclic voltammetry (FSCV) has become the preferred method for in vivo electrochemical measurements because the cyclic votammogram fingerprint aids in analyte identification and the temporal resolution is $100 \mathrm{~ms}$, allowing neurotransmitter measurements on a subsecond time scale. ${ }^{18-21}$ CFMEs are commonly coupled with FSCV for the detection of dopamine; however, dopamine signal decreases with increasing scan repetition frequency at CFMEs. ${ }^{22,23}$ Thus, measurements are typically collected at $10 \mathrm{~Hz}$ as a compromise between temporal resolution and sensitivity. While methods to improve temporal 
resolution by raising the scan rate have been explored, the signal still decreases with increasing scan repetition frequency. ${ }^{24,25}$ In contrast, our group has recently discovered that at CNTYMEs, the dopamine signal does not change significantly with increasing scan repetition frequency, which facilitates a faster scan repetition frequency using $\mathrm{FSCV} .{ }^{9}$ Since the CNT yarn is a soft material, polishing it to make an elliptical disk microelectrode enriches the edge plane of vertically aligned CNTs. However, the disk geometry limits the surface area and sensitivity.

Electrode sensitivity can be improved at carbon based microelectrodes by several surface modification methods, including acid treatment ${ }^{12}$, electrochemical activation ${ }^{3}$, polymer coating ${ }^{26-}$ ${ }^{28}$, and metal nanoparticle coating ${ }^{29,30}$. Strein and Ewing reported laser activation as a simple way to increase the sensitivity for voltammetry at disk CFMEs. ${ }^{31,32}$ Laser activation increases the surface area and improves the sensitivity as well as electron transfer kinetics. However, laser activation has not been studied at CNT based electrodes and its effect on surface properties has not been characterized.

In this paper, we laser treated CNTYMEs to improve the detection sensitivity to dopamine while maintaining the high signals at rapid scan repetition frequencies. By applying 15 pulses laser treatment on CNTYMEs surface, the sensitivity improved by three fold, and LOD was enhanced to $13 \pm 2 \mathrm{nM}$. The surface properties of laser-treated electrodes were characterized in order to understand the enhanced electrochemical response. The surface area and the amount of oxygen containing functional groups increased, which would lead to more adsorption sites for dopamine. In addition, the high surface roughness at CNTYMEs could lead to trapping of the oxidation product of dopamine, dopamine-o-quinone, causing the increased reversibility and scan repetition frequency independent response. Laser-treated CNTYMEs were applied for the detection of neurotransmitters in vivo for the first time and could be a potential alternative to CFMEs in the future. 


\subsection{Experimental Section}

\subsubsection{Solutions}

Dopamine hydrochloride and ascorbic acid were purchased from Sigma-Aldrich (St. Louis, MO). A $10 \mathrm{mM}$ stock solution was prepared in $\mathrm{HClO}_{4}$, and were diluted daily to the desired concentration in phosphate buffered saline (131.3 mM NaCl, $3.00 \mathrm{mM} \mathrm{KCl}, 10 \mathrm{mM} \mathrm{NaH}_{2} \mathrm{PO}_{4}, 1.2$ $\mathrm{mM} \mathrm{MgCl}_{2}, 2.0 \mathrm{mM} \mathrm{Na}_{2} \mathrm{SO}_{4}$, and $1.2 \mathrm{mM} \mathrm{CaCl}_{2}$ with the $\mathrm{pH}$ adjusted to 7.4).

\subsubsection{Electrochemistry}

FSCV was performed with a ChemClamp potentiometer (Dagan, Minneapolis, MN, with 1 MOhm Headstage). The waveform was generated and the data was collected using a High Definition Cyclic Voltammetry (HDCV) breakout box, HDCV analysis software program (UNC Chemistry Department, Electronics Design Facility) and PCle-6363 computer interface cards (National Instruments, Austin, TX). Electrodes were backfilled with $1 \mathrm{M} \mathrm{KCl}$ and a silver wire was inserted to connect the electrode to the potentiostat headstage. The typical triangular waveform swept the applied potential from $-0.4 \mathrm{~V}$ to $1.3 \mathrm{~V}$ at $400 \mathrm{~V} / \mathrm{s}$ versus an $\mathrm{Ag} / \mathrm{AgCl}$ reference electrode, at a scan repetition frequency of $10 \mathrm{~Hz}$. The repetition rate was varied for some experiments.

Electrodes were tested using a flow-injection system, as previously described. Analyte was injected for 5 seconds and current versus time traces were obtained by integrating the current in a $100 \mathrm{mV}$ window centered at the oxidation peak for each cyclic voltammogram (CV). Background-subtracted CVs were calculated by subtracting the average of 10 background scans, taken before the compound was injected, from the average of five CVs recorded after the analyte bolus was injected. 


\subsubsection{Carbon Nanotube Yarn Microelectrode Preparation}

A $0.68 \mathrm{~mm}$ ID $\times 1.2 \mathrm{~mm}$ OD (A-M Systems, Carlsborg, WA) glass capillary was pulled into a glass pipet puller and cut to have an opening diameter of $\sim 50 \mu \mathrm{m}$. A piece of commercially available CNTY (10-25 $\mu \mathrm{m}$ in diameter, 1-2 cm long, General Nano, LLC, Cincinnati, OH) was inserted into the glass pipette. The electrodes were epoxied with Epon Resin 828 (MillerStephenson, Danbury, CT) mixed with 14\% (w/w) 1,3-phenylenediamine hardener (Sigma-Aldrich, St. Louis, MO) heated to $85^{\circ} \mathrm{C}$. The epoxied electrodes were cured overnight at room temperature and then heated at $100^{\circ} \mathrm{C}$ for $2 \mathrm{~h}$ and at $150^{\circ} \mathrm{C}$ overnight. Electrodes were polished at a $45^{\circ}$ angle on a fine diamond abrasive plate (Sutter Instruments model BV-10, Novato, CA) to create an elliptical active area. Cylindrical CFMEs were fabricated using 7- $\mu$ m-diameter T-650 carbon fibers (Cytec Technologies, Woodland Park, NJ). ${ }^{9}$

\subsubsection{Laser Treatment}

A KrF 248nm pulsed excimer laser (Lambdaphysik Lasertechnik LPX305 IMC, Coherent Inc., CA, 20 ns full width at half maximum) with repetition rate of $1 \mathrm{~Hz}$ was used to irradiate the samples. The laser spot size and energy density were $2 \times 2 \mathrm{~mm}^{2}$ and $50 \mathrm{~mJ} / \mathrm{cm}^{2}$, respectively.

\subsubsection{Surface Characterization}

Scanning electron microscope (SEM) images were taken on Merlin field emission SEM (Zeiss, Thornwood, NY) with a secondary electron detector using an accelerating voltage of $2 \mathrm{kV}$ and a working distance of $5.0 \mathrm{~mm}$. Raman spectroscopy measurements were performed with a Renishaw 100 confocal micro-Raman system (Renishaw, Hoffman Estates, IL) with a 1800 lines/mm diffraction grating, $532 \mathrm{~nm}$ laser focused to a spot size of about $2 \mu \mathrm{m}$ through a 100x objective, and a Peltier-cooled charge-coupled device detector. Three-dimensional laser (violet 
laser, $408 \mathrm{~nm}$ ) scanning confocal microscopy (VK-X, Keyence, IL) was performed to measure the surface roughness.

\subsubsection{In Vivo Measurements}

Male Sprague-Dawley rats (250-350 g) purchased from Charles River were housed in a vivarium and given food and water ab libitum. All experiments were approved by the Animal Care and Use Committee of the University of Virginia. The rat was anesthetized with urethane (1.5 $\mathrm{mg} / \mathrm{kg}$ i.p.), the scalp shaved, and $0.25 \mathrm{~mL}$ bupivicaine ( $0.25 \%$ solution) given subcutaneously. The working electrode was implanted in the caudate putamen (in mm from bregma: AP + 1.2, ML + 2.0, and DV -4.5 to 5.0 ), the stimulating electrode in the substantia nigra ( $A P-5.4, M L+1.2$, and $\mathrm{DV}-8.0$ ), and the $\mathrm{Ag} / \mathrm{AgCl}$ reference electrode in the contralateral side of the brain. The DV placement of the stimulating electrode was adjusted downward until a robust dopamine signal was measured. After implantation in the brain, the FSCV waveform was applied to the microelectrode for 30 min to allow the electrode to stabilize and the brain to recover. Stimulated release was electrically evoked using biphasic stimulation pulses $(300 \mu \mathrm{A}, 120$ pulses, $60 \mathrm{~Hz})$. For in vivo measurements, the scan repetition frequency was either $10 \mathrm{~Hz}$ or $50 \mathrm{~Hz}$, which was chosen to avoid overlap with the electrical stimulation at $60 \mathrm{~Hz}$.

\subsubsection{Statistics}

All values are given as mean \pm standard error of the mean (SEM) for $n$ number of electrodes and all error bars are SEM. Paired or unpaired t tests were performed to compare properties between two groups. A one-way ANOVA with Bonferonni post-tests was used to compare effects among multiple groups. All statistics were performed in GraphPad Prism6 (GraphPad Software,Inc., La Jolla, CA). Scanning electron microscopy images were processed using ImageJ (Rasband, W.S., National Institutes of Health, Bethesda, MD,). 


\subsection{Results and Discussion}

\subsubsection{Laser Treatment Improves Carbon Nanotube Yarn Microelectrode Signals}

CNTYMEs were laser treated by a KrF $248 \mathrm{~nm}$ pulsed excimer laser with a pulse duration of $20 \mathrm{~ns}$ and power of $50 \mathrm{~mJ} / \mathrm{cm}^{2}$. The response to $1 \mu \mathrm{M}$ dopamine was tested using FSCV, with the electrode scanned from -0.4 to $1.3 \mathrm{~V}$ and back at $400 \mathrm{~V} / \mathrm{s}$ and a scan repetition frequency of $10 \mathrm{~Hz}$. Figure 2.1 shows the example CVs from the same CNTYME before and after 15 laser pulses. Both the oxidation and reduction currents for $1 \mu \mathrm{M}$ dopamine increased about three fold after laser treatment (Figure 3.1A) and the separation between the oxidation and reduction peak potential $\left(\Delta \mathrm{E}_{\mathrm{p}}\right)$ decreased. ${ }^{33}$ There was no delay in the response to a bolus of dopamine, as seen on the current versus time curve after laser treatment (Figure 3.1B), which indicates the lasertreated CNTYME maintains good temporal resolution with increased sensitivity. Figure 3.1C shows the background current increased about two-fold, smaller than the increase in oxidation and reduction currents.
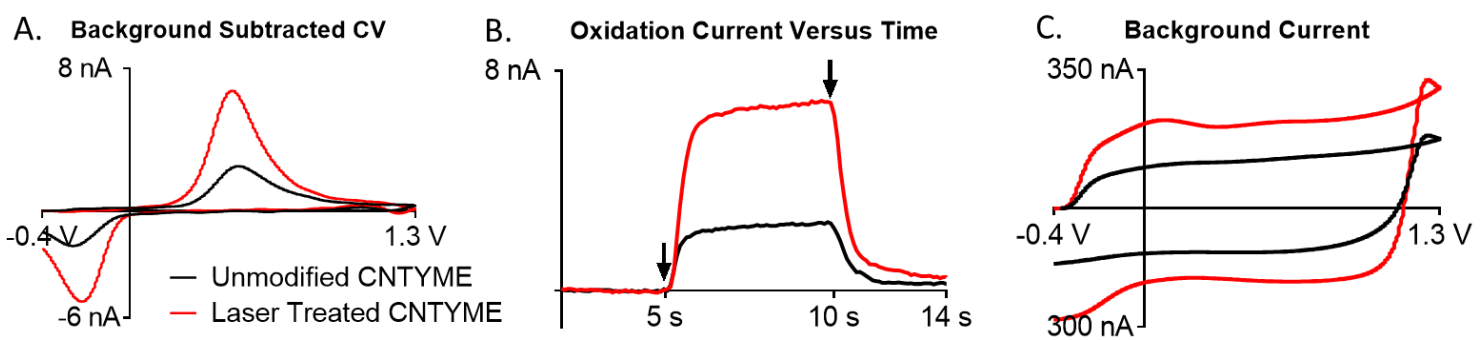

Figure 3.1: Comparison of the response to $1 \mu \mathrm{M}$ dopamine at an unmodified disk CNTYME (black line) and the same electrode after 15 pulses laser treatment (red line). Electrochemical response was measured with scan rate of $400 \mathrm{~V} / \mathrm{s}$ and scan repetition frequency of $10 \mathrm{~Hz}$. (A) Background subtracted cyclic voltammograms to $1 \mu \mathrm{M}$ dopamine, (B) measured oxidation current versus time for a flow injection analysis experiment (dopamine bolus injection and changing back to PBS buffer are marked as black arrows), and (C) background currents in PBS buffer.

CNTYMEs were treated with three different laser pulse counts $(5,10$, and 15 pulses) to optimize the laser treatment. The ratio of oxidation current after laser treatment to before was 3.2 $\pm 0.5(n=4)$ for 15 laser pulses, which is significantly larger than the ratio obtained for 5 pulses 
$(2.1 \pm 0.2, n=4)$ and 10 pulses $(2.0 \pm 0.2, n=4)$ (unpaired t-tests, $p<0.01)$. Preliminary tests with more than 15 pulses found that the surface was burned and sensitivity did not further increase. Fifteen laser pulses was chosen as optimal for further electrochemical and surface characterization experiments.

Table 3.1 summarizes the effects of laser treatment on the signals for $1 \mu \mathrm{M}$ dopamine. Both the oxidation and reduction currents increased significantly after laser treatment (paired ttest, $p<0.01$ and $p<0.05$ for oxidation and reduction, respectively) and the LOD improved significantly from $25 \pm 2 \mathrm{nM}$ to $13 \pm 2 \mathrm{nM}$ (paired t-test, $\mathrm{p}<0.05$ ). Although the increase in the background current was significant (paired t-test, $p<0.01$ ), the background current increased by only 2-fold after laser treatment, which is smaller than the 3-fold increase in the Faradaic current. The increase in background current is typically proportional to the increase in surface area. Because the increase in Faradaic currents is greater than the increase in the background currents, the increasing sensitivity is likely due to both increasing the surface area and enriching the number of active adsorption sites. ${ }^{32,33}$

Table 3.1: Comparison of dopamine detection at CNTYMEs and laser treated CNTYMEs.

\begin{tabular}{|c|c|c|c|c|c|c|}
\hline & $\begin{array}{c}\text { Oxidation } \\
\text { Current } \\
(\mathrm{nA})\end{array}$ & $\begin{array}{c}\text { Reduction } \\
\text { Current } \\
(\mathrm{nA})\end{array}$ & $\mathrm{LOD}(\mathrm{nM})$ & $\begin{array}{c}\text { Background } \\
\text { Current }(\mathrm{nA})\end{array}$ & $\begin{array}{c}\text { Reduction/ } \\
\text { Oxidation } \\
\text { Current } \\
\text { Ratio }\end{array}$ & $\Delta \mathrm{E}_{\mathrm{p}}(\mathrm{V})$ \\
\hline $\begin{array}{c}\text { Unmodified } \\
\text { CNTYMEs } \\
(\mathrm{n}=12)\end{array}$ & $1.9 \pm 0.3$ & $1.4 \pm 0.2$ & $25 \pm 2$ & $86 \pm 6$ & $0.74 \pm 0.02$ & $0.70 \pm 0.01$ \\
\hline $\begin{array}{c}\text { Laser- } \\
\text { Treated } \\
\text { CNTYME } \\
(\mathrm{n}=12)\end{array}$ & $6 \pm 1^{* *}$ & $4.6 \pm 0.9^{*}$ & $13 \pm 2^{*}$ & $161 \pm 18^{* *}$ & $0.70 \pm 0.03$ & $0.66 \pm 0.01^{* *}$ \\
\hline $\begin{array}{c}\text { Treated/ } \\
\text { Unmodified }\end{array}$ & $3.0 \pm 0.7$ & $3.2 \pm 0.8$ & $0.53 \pm 0.09$ & $1.9 \pm 0.2$ & $0.95 \pm 0.05$ & $0.94 \pm 0.02$ \\
Ratio $(\mathrm{n}=12)$ & & & & & & \\
\hline
\end{tabular}

Oxidation, reduction, reduction/oxidation ratios, and $\Delta \mathrm{E}_{\mathrm{p}}$ are for $1 \mu \mathrm{M}$ dopamine detection. Electrochemical measurements were performed with a typical FSCV waveform scanning from $0.4 \mathrm{~V}$ to $1.3 \mathrm{~V}$ and back at $400 \mathrm{~V} / \mathrm{s}$, with scan repetition frequency of $10 \mathrm{~Hz}$.

Significantly different than unmodified: ${ }^{* *}$ paired t-test, $p \leq 0.01$, * paired t-test, $p \leq 0.05$ 
The reduction to oxidation current ratio is a measure of the reversibility of dopamine redox reaction. ${ }^{7}$ Although the reduction/oxidation current ratios for unmodified and laser-treated CNTYMEs were not significantly different (paired t-test, $p=0.22$ ), they were both larger than the ratio for CFMEs $(0.63 \pm 0.01$, unpaired t-test, $p<0.01$ for both unmodified and laser-treated CNTYME) ${ }^{9}$. This higher ratio indicates stronger adsorption and slower desorption kinetics of dopamine-o-quinone (DOQ, oxidation product of dopamine) at the nanotubes, causing more DOQ to be recycled back to dopamine on the electrode surface where it may be oxidized again. Therefore, dopamine oxidation is more reversible at both laser-treated and untreated CNTYMEs than CFMEs. ${ }^{9}$ The $\Delta \mathrm{E}_{\mathrm{p}}$ observed after laser treatment was significantly smaller, indicating an increase in the rate of electron transfer.

Microelectrodes are typically used in vivo for hours at a time to measure neurotransmission in behavioral or pharmacological experiments. ${ }^{34-36}$ Figure $3.2 \mathrm{~A}$ shows the dopamine oxidation signal is constant for four hours of continuous scanning and that the laser-treated surface is stable over the time length of a biological experiment. With electrodes tested for longer than 4 hours, there was no evidence of signal degradation. To test long-term stability, electrochemical tests were repeated over 15 days and no significant decrease in current was observed (Figure 3.2B). Laser-etched CNTYMEs are stable for several hours of experimental use and shelf stable, allowing them to be made in batches. 


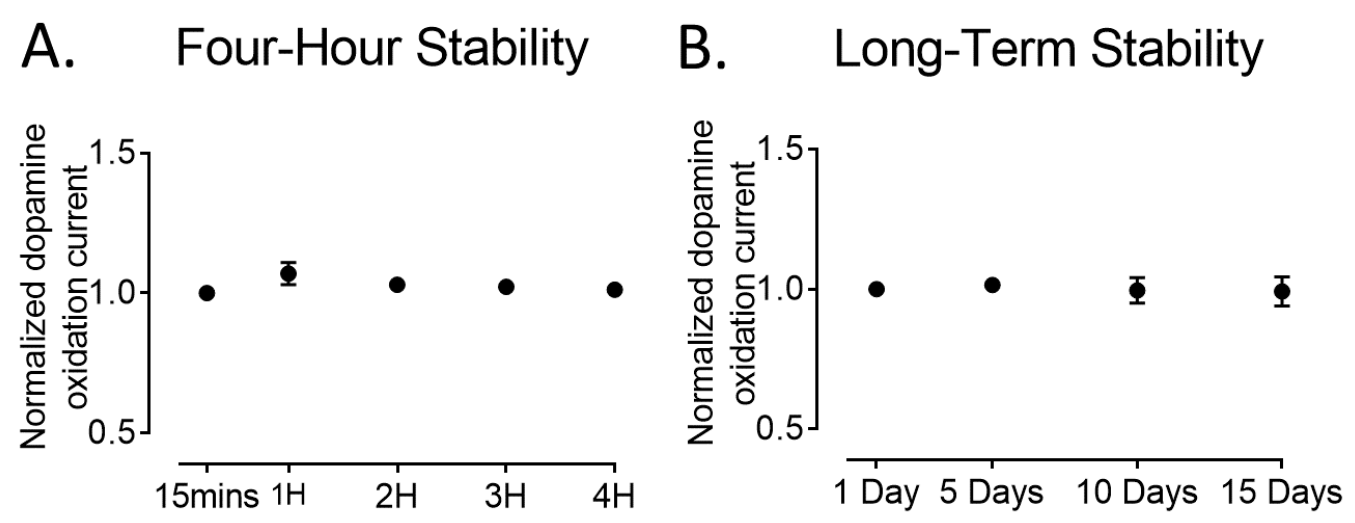

Figure 3.2: Stability test at laser treated CNTYMEs. (A) Four hour $(n=4)$ and (B) long term $(n=7)$ stability test of laser treated CNTYMEs. Oxidation current to $1 \mu \mathrm{M}$ dopamine was normalized to the signal observed after 15 minutes equilibration. Electrochemical response was measured with scan rate of $400 \mathrm{~V} / \mathrm{s}$ and scan repetition frequency of $10 \mathrm{~Hz}$. Error bar is standard error of mean.

Ascorbic acid is one of the most abundant antioxidants and often coexists with dopamine in biological samples. ${ }^{37}$ The oxidation potential for ascorbic acid can be similar to dopamine. ${ }^{38}$ The selectivity of dopamine to ascorbic acid was measured for CNTYMEs, and example CVs were shown in Figure 3.3A-B. The ratio of the oxidation current for $1 \mu \mathrm{M}$ dopamine to $200 \mu \mathrm{M}$ ascorbic was $0.15 \pm 0.01$ at unmodified CNTYMEs and $0.43 \pm 0.11$ after laser treatment (Figure 3.3C, unpaired t-test, $p<0.05, n=6$ ). The larger ratio after laser treatment indicates that laser treated CNTYMEs have higher selectivity for dopamine over ascorbic acid, due to the abundant functional groups introduced. 
A. Unmodified CNTYME

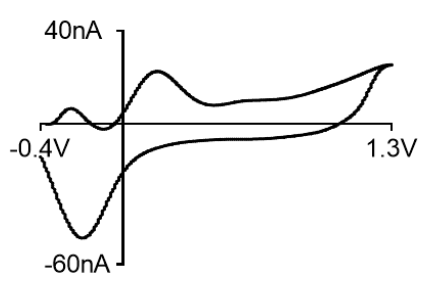

B. Laser Treated CNTYME

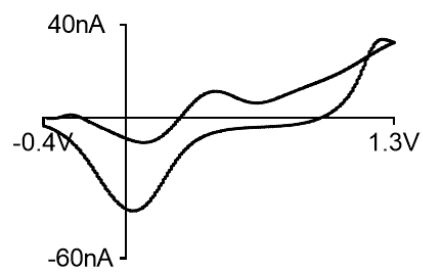

C.

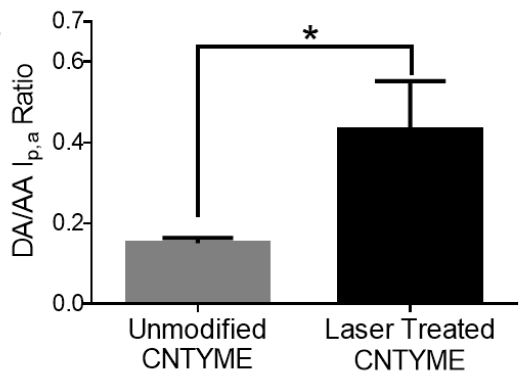

Figure 3.3: Example cyclic voltammograms of $200 \mu \mathrm{M}$ ascorbic acid on an (A) unmodified CNTYME and (B) laser-treated CNTYME after 15 min equilibration in PBS buffer solution using a waveform of -0.4 to $1.3 \mathrm{~V}$ and back at $400 \mathrm{~V} / \mathrm{s}$, with sample scanning frequency of $10 \mathrm{~Hz}$. (C) The ratio of oxidation current of $1 \mu \mathrm{M}$ dopamine over $200 \mu \mathrm{M}$ ascorbic acid at unmodified CNTYME $(0.15 \pm 0.01)$ and laser-treated CNTYME $(0.43 \pm 0.11) .{ }^{*}$ indicates the ratios are significant different (unpaired t-test, $\mathrm{p}<0.05, \mathrm{n}=6$ ).

\subsubsection{Improved Temporal Resolution for Dopamine Detection at Laser-Treated Carbon}

Nanotube Yarn Microelectrodes

The typical FSCV waveform with a scan rate of $400 \mathrm{~V} / \mathrm{s}$ takes $8.5 \mathrm{~ms}$. The time between waveforms is $91.5 \mathrm{~ms}$ with a scan repetition frequency of $10 \mathrm{~Hz}$ but only $11.5 \mathrm{~ms}$ when the scan repetition frequency increases to $50 \mathrm{~Hz}$. While the dopamine signal drops with decreasing time for dopamine adsorption at CFMEs, ${ }^{22}$ our group previously reported that the current at CNTYMEs is scan repetition frequency independent due to different adsorption/desorption kinetics. ${ }^{9}$ To study whether laser-treated CNTYMEs were scan repetition frequency independent, the current for dopamine was measured with scan repetition frequencies from $10 \mathrm{~Hz}$ to $100 \mathrm{~Hz}$ (Fig. 3.4A). The oxidation current drop was dramatic at CFMEs, with approximately $50 \%$ signal loss at $50 \mathrm{~Hz}$ and $67 \%$ loss at $100 \mathrm{~Hz}$ compared to $10 \mathrm{~Hz}$. The oxidation current of dopamine at unmodified CNTYMEs only dropped approximately $15 \%$ at $50 \mathrm{~Hz}$ and $25 \%$ at $100 \mathrm{~Hz}$. In comparison, the current at laser-treated CNTYMEs dropped $6 \%$ at $50 \mathrm{~Hz}$ and $21 \%$ at $100 \mathrm{~Hz}$. Although the electrode type significantly affected the frequency response (one-way ANOVA, $p<0.0001$ ), the frequency response of the unmodified and laser treated CNTYMEs were not significantly different 
(one-way ANOVA, Bonferroni's multiple comparisons test, $p=0.1522$ ). Figure 3.4B shows example, normalized CVs taken at $10 \mathrm{~Hz}$ and $50 \mathrm{~Hz}$ at these three different electrode materials.
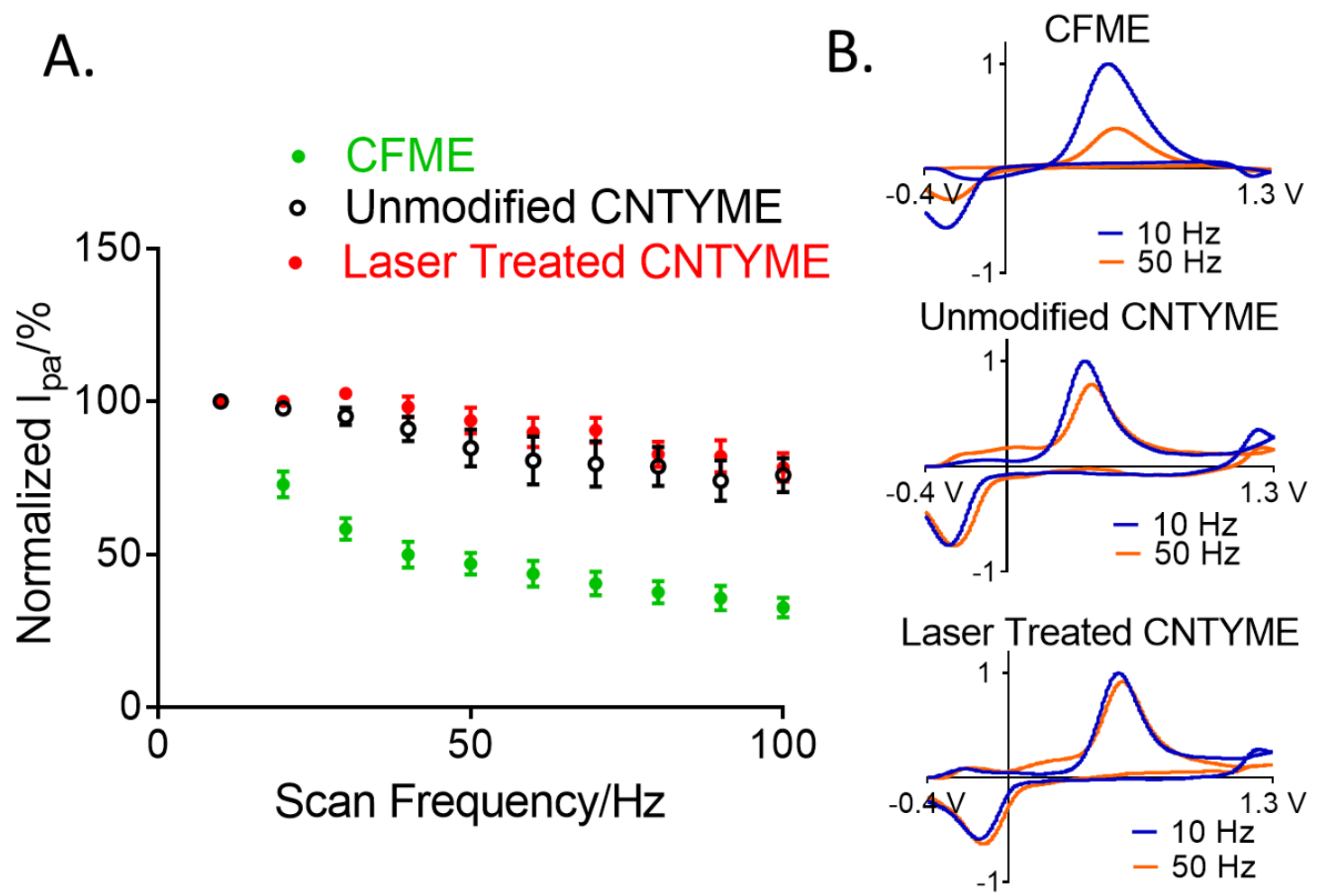

Figure 3.4: Effect of scan repetition frequency for $1 \mu \mathrm{M}$ dopamine detection at CFMEs, unmodified and laser treated CNTYMEs: $(A)$ peak oxidation current at CFMEs (green dot, $n=5$ ), unmodified CNTYMEs (black dot, $n=5$ ), and laser treated CNTYMEs (red dot, $n=5$ ) with $-0.4-$ $1.3 \mathrm{~V}$ waveform and scan rate of $400 \mathrm{~V} / \mathrm{s}$. Peak currents were normalized to the current at $10 \mathrm{~Hz}$, and error bars represent the standard error of the mean. (B) Example normalized CVs of $1 \mu \mathrm{M}$ dopamine bolus injection at unmodified CNTYME, laser treated CNTYME, and a CFME at $10 \mathrm{~Hz}$ (blue line) and $50 \mathrm{~Hz}$ (orange line) scan repetition frequency. CVs are normalized to the $10 \mathrm{~Hz}$ signal.

To determine the applicability of laser-treated CNTYMEs as in vivo sensors with fast temporal resolution, stimulated dopamine release was measured in anesthetized rats. Stimulation pulse trains were applied $(300 \mu \mathrm{A}, 120$ pulses, $60 \mathrm{~Hz})$ to the dopamine cell bodies and the dopamine release recorded in the caudate-putamen near the terminals. Dopamine measurements at CNTYMEs were reported in acute brain slices by Sombers' group; ${ }^{11}$ but this is the first use of CNTYMEs for in vivo measurement of dopamine. Figure 3.5 compares dopamine 
release in vivo at a CFME (Fig. 3.5A) and a laser-treated CNTYME (Fig. 3.5B) with scan repetition frequency of $10 \mathrm{~Hz}$ (blue line) and $50 \mathrm{~Hz}$ (orange line). The general shape of the $\mathrm{CVs}$ are similar, but like the calibration studies, the laser-treated CNTYME CV was more reversible, with a larger reduction peak, and had a smaller $\Delta \mathrm{E}_{\mathrm{p}}$ than the CFME. Oxidation current of stimulated dopamine at $50 \mathrm{~Hz}$ was $45 \pm 2 \%$ of the $10 \mathrm{~Hz}$ value at CFMEs, which was significantly smaller than lasertreated CNTYMEs, where it was $75 \pm 5 \%$ (Fig. $3.5 \mathrm{C}$, unpaired t-test, $\mathrm{p}<0.001, \mathrm{n}=5$ ). The in vivo measurements indicate laser-treated CNTYMEs maintained relatively high dopamine sensitivity with $50 \mathrm{~Hz}$ scan repetition frequency compared to CFMEs, consistent with in vitro data.

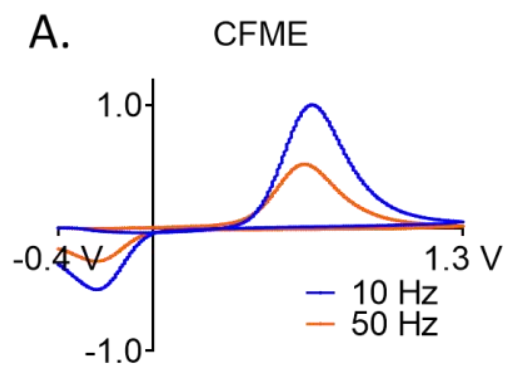

B. Laser Treated CNTYME

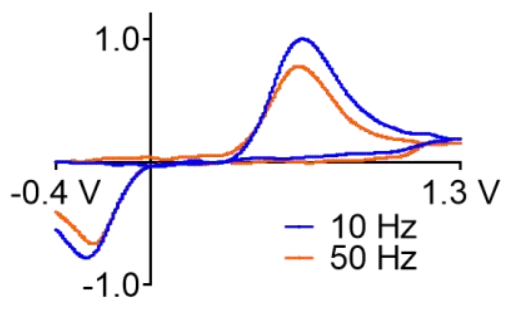

C. I I,a Ratio In Vivo $(50 \mathrm{~Hz} / 10 \mathrm{~Hz})$

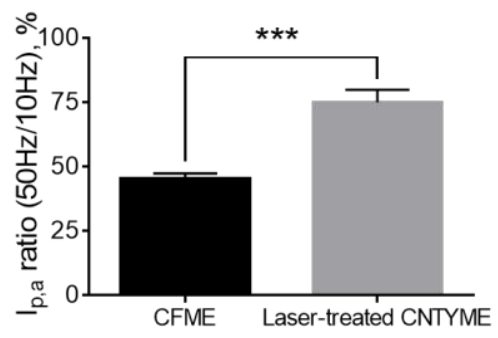

Figure 3.5: Comparison of the effect of FSCV scan repetition frequency in vivo. Example normalized CVs of stimulated dopamine release at (A) a CFME and (B) a laser etched CNTYME in vivo, with typical FSCV waveform scanning from $-0.4 \mathrm{~V}$ to $1.3 \mathrm{~V}$ and back at $400 \mathrm{~V} / \mathrm{s}$, and scan repetition frequency of $10 \mathrm{~Hz}$ (blue line) and $50 \mathrm{~Hz}$ (orange line). (C) The ratio of oxidation current for stimulated release with $50 \mathrm{~Hz}$ to $10 \mathrm{~Hz}$ scan repetition frequency at CFMEs $(n=6)$ and lasertreated CNTYMEs $(n=6)$. Stimulated dopamine release was detected in the caudate putamen with a stimulation pulses train of 120 pulses at $60 \mathrm{~Hz}$. Error bars are standard error of mean. ${ }^{* * *}$ $p<0.001$ (unpaired t-test)

\subsubsection{Surface Characterization of Laser-Treated Carbon Nanotube Yarn Microelectrode}

Figure 3.6 shows scanning electron microscope (SEM) images of the ends of both unmodified (Figure 3.6A) and laser-treated CNTYMEs (Figure 3.6B). The spikes on the unmodified CNTYME, with a diameter of about $30 \mathrm{~nm}$, suggest the surface consists primarily of multi-walled CNT ends. The CNT bundles are laying down on the unmodified CNTYME but are standing up straighter after laser treatment. 

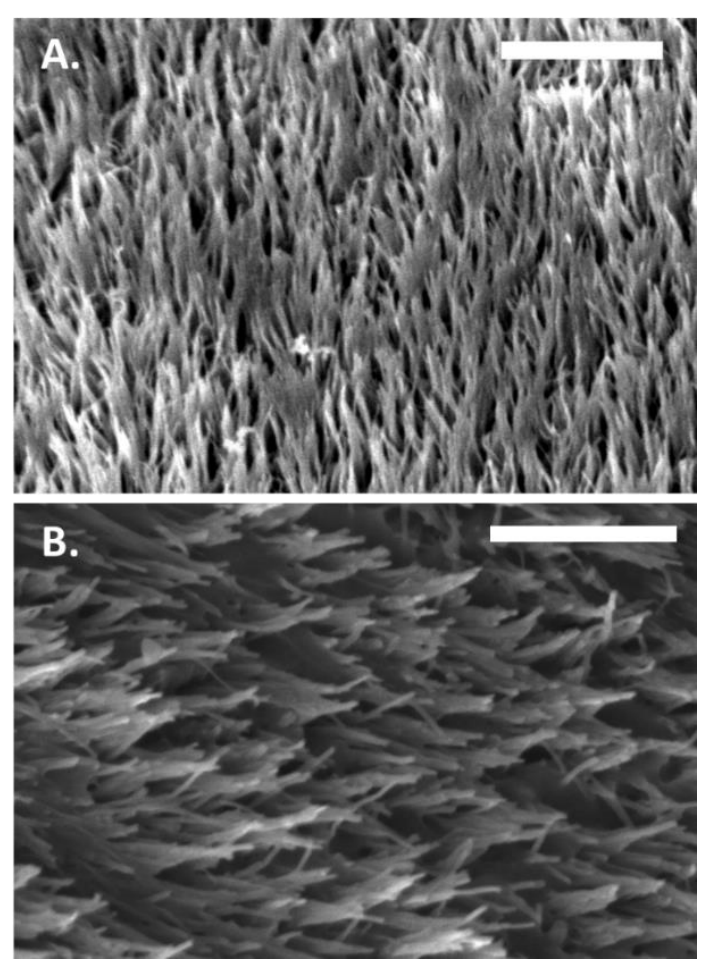

Figure 3.6: SEM images of (A) unmodified CNTYME and (B) laser treated CNTYME. Scale bar: $500 \mathrm{~nm}$.

The SEMs imply a greater surface roughness at laser-treated CNTYMEs and threedimensional laser scanning confocal microscopy was used to measure the surface roughness precisely. Laser scanning confocal microscopy has a larger measuring range than atomic force microscopy and is better for surfaces with micrometer-scaled roughness. ${ }^{39}$ In addition, laser scanning confocal microscopy does not require a leveled surface, which is useful for examining beveled electrodes. Figure 3.7 displays the surface profiles and associated line plots (from the white dashed line on the profile) for a CFME, an unmodified CNTYME, and the same CNTYME after laser treatment. The surface roughness of the CNTYME was greater than that of the CFME and the roughness increased after laser treatment. The mean roughness depth $\left(R_{z}\right)$, defined as the average distance between the highest peak and lowest valley in each sampling length, is a general description of the height variations in the surface. The surface roughness was significantly larger at both unmodified and laser-treated CNTYMEs compared to CFMEs (Table 3.2, one-way 
ANOVA, Bartlett's test, $p<0.01$ ), which is consistent with previous study. ${ }^{11} R_{z}$ is significantly larger at laser-treated CNTYMEs than the same electrodes before laser treatment (Table 3.2, paired t-test, $\mathrm{p}<0.05)$.

Table 3.2: Surface properties of CNTYMEs. The mean roughness depth $\left(R_{z}\right), D / G$ ratio from Raman spectra, and relative oxygen content at CFME, unmodified CNTYMEs and the same CNTYMEs after laser treatment.

\begin{tabular}{|c|c|c|c|}
\hline & $\mathrm{R}_{\mathrm{z}} / \mathrm{nm}(\mathrm{n}=6)$ & $\begin{array}{c}\text { Raman D/G Ratio } \\
(\mathrm{n}=4)\end{array}$ & $\begin{array}{c}\text { Oxygen Content/\% } \\
(\mathrm{n}=4)\end{array}$ \\
\hline CFME & $420 \pm 30$ & $3.0 \pm 0.2$ & $4.5 \pm 0.4$ \\
\hline Unmodified CNTYME & $1910 \pm 190$ & $0.78 \pm 0.01$ & $10 \pm 1$ \\
\hline $\begin{array}{c}\text { Laser-Treated } \\
\text { CNTYME }\end{array}$ & $3300 \pm 660$ & $0.81 \pm 0.02$ & $14 \pm 2$ \\
\hline
\end{tabular}


A. CFME

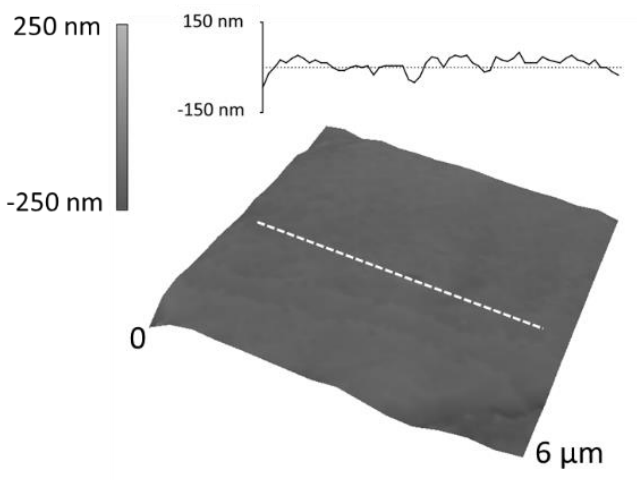

B. Unmodified CNTYME

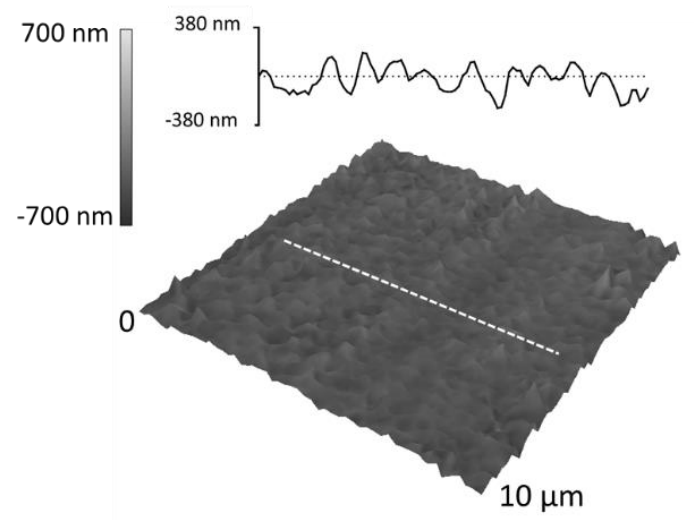

\section{Laser-treated CNTYME}

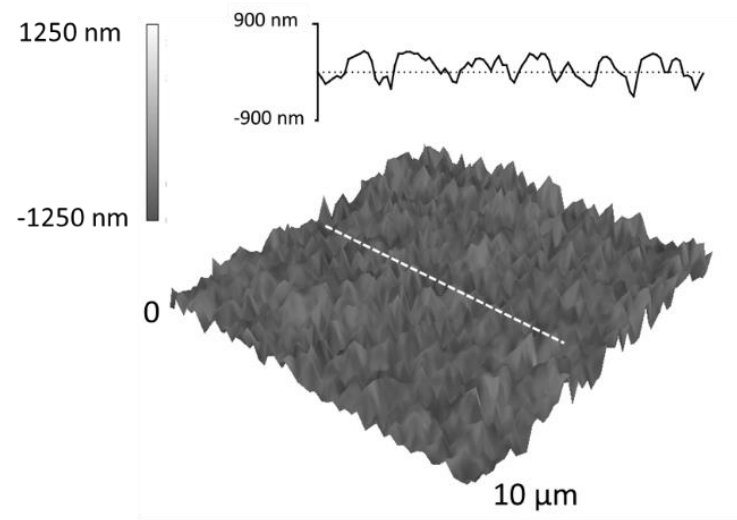

Figure 3.7: Representative three-dimensional laser confocal profile image of (A) CFME, (B) unmodified CNTYME, and $(C)$ the same microelectrode after laser treatment. Insets indicate the associated line plot from each sample. Note the scales are different for the different panels. 
One implication of greater surface roughness is that the mass transport profiles would be different at CNTYMEs than CFMEs. The high density of uneven CNTs at polished CNTYME surfaces could lead to thin-film diffusion mass transport profiles, which would trap more DOQ at the surface where it can reduced back to dopamine. ${ }^{40,41}$ The diffusion layer thickness, $\delta$, can be approximated by $\delta=\sqrt{2 D t}$, where $\mathrm{D}$ is the diffusion coefficient, and $t$ is the length of time for diffusion. Assuming a diffusion coefficient of DOQ equal to that of dopamine $\left(6 \times 10^{-6} \mathrm{~cm}^{2} / \mathrm{s}\right),{ }^{23}$ and that DOQ exists for about $40 \%$ of the total time of one single waveform (3.3 ms for $400 \mathrm{~V} / \mathrm{s}),{ }^{22}$ the diffusion layer thickness of DOQ is approximately $1900 \mathrm{~nm}$. This thickness is the same order of magnitude as $R_{z}$ for unmodified CNTYMEs and about half of the $R_{z}$ for laser-treated CNTYMEs. Thus, on the timescale of the experiment, the DOQ diffusion distance is similar to the depth of the crevices, and DOQ may be trapped in the surface crevices for a short amount of time. Another possible effect of laser treatment on mass transport would be that laser treatment would decouple the CNTs, leading them to act as nanoelectrodes in parallel instead of a collective microelectrode. This decoupling is evidenced by the increased background capacitive current. Acting as nanoelectrodes in parallel would change the dopamine diffusion pattern from planar to radial diffusion, ${ }^{42,43}$ which would enhance mass transfer and enable rapid and highly sensitive detection of dopamine.

Our previous work on CNTYMEs showed that the key to maintain a scan repetition frequency independent response is that the desorption rate for $D O Q$ is similar to that of dopamine. ${ }^{9}$ If the DOQ is trapped because of the greater surface roughness, this could lead to more DOQ being available for adsorption. ${ }^{9}$ Although the difference of mass transport caused by the different degree of surface roughness has significant effects on millisecond scale diffusion, the current vs time response curve was not significantly slowed, as shown in Figure 3.1B. Thus, the electrodes still respond quickly to dopamine, with an average $10 \%$ to $90 \%$ signal rise time of $1.0 \pm 0.2 \mathrm{~s}$ with $10 \mathrm{~Hz}$ and $0.9 \pm 0.2 \mathrm{~s}$ with $50 \mathrm{~Hz}$ at laser treated CNTYMEs $(n=5)$, compared to $1.2 \pm 0.1 \mathrm{~s}$ at CFMEs. ${ }^{26}$ 
Table 3.2 also summarizes the $\mathrm{D}$ to $\mathrm{G}$ peak ratio from Raman spectra obtained at CFMEs, unmodified CNTYMEs, as well as the same CNTYMEs after laser treatment. The ratio of the D and $G$ band area is widely used to evaluate the quality of carbon nanomaterials. ${ }^{44,45}$ The D/G ratio at CFMEs was much larger than both unmodified and laser treated CNTYMEs due to the higher disorder of carbon fibers than CNTs (unpaired t-test, $p<0.001$ for both unmodified and lasertreated CNTYMEs). ${ }^{33,46}$ The $\mathrm{D} / \mathrm{G}$ ratios did not significantly change after laser treatment at CNTYMEs (paired t-test, $\mathrm{p}=0.53$ ), indicating the ratio of edge plane $\left(\mathrm{sp}^{3}\right.$ hybridized) carbon to basal plane ( $\mathrm{sp}^{2}$ hybridized) carbon does not change significantly. However, because the surface area is greater after laser treatment, there may be an increase in both edge plane and basal plane sites, and the overall amount of defects may increase, which could be important for dopamine adsorption.

The oxygen content was measured by energy dispersive X-ray spectroscopy (EDS) before and after laser treatment on the same CNTYME. The oxygen content significantly increased after laser treatment (Table 3.2, paired t-test, $p<0.05$ ). In addition, the oxygen content at both unmodified and laser-treated CNTYMEs was significantly larger than that of CFMEs (unpaired t-test, $\mathrm{p}<0.01$ for both). The laser-treated CNTYME surface has abundant oxygen containing functional groups which provide more adsorption sites for dopamine. Oxygen containing functional groups, such as quinones, hydroxyl, and carboxylic acids are known to be negatively charged at physiological $\mathrm{pH}$ and have electrostatic interactions with positively charged dopamine. ${ }^{47}$ Therefore, the laser treatment not only creates more surface roughness but also oxidizes the edge plane carbon to a greater degree, contributing to the high sensitivity to dopamine.

\subsubsection{Correlation of Electrochemical and Surface Properties}

Correlating surface states and electrochemical properties is difficult, but the differences between CFMEs, CNTYMEs, and laser-etched CNTYMEs allow some generalizations of how the 
surface affects the electrochemical performance. Disk CNTYMEs have a much larger surface area than disk CFMEs, which would allow more surface for dopamine adsorption. The mean surface roughness of CNTYMEs is on the same order of magnitude as the diffusion distance, so dopamine and DOQ are more likely to get trapped near the surface, leading to better reversibility. Additionally, the main reason that the signal decreases with increasing scan repetition frequency for CFMEs is that DOQ desorbs and diffuses away from the electrode. At the rough CNTYME surface, the DOQ would remain close to the surface if it desorbs and thus could more easily absorb again, and be reduced back to dopamine. Therefore, the electrochemical data are not only scan repetition frequency independent, but much more reversible at CNTYMEs compared to CFMEs.

The sensitivity at CNTYMEs is not high because they are disk electrodes and typical in vivo measurements are made with larger surface area cylindrical electrodes. The surface characterization data indicate that laser etching increases the surface roughness and oxygen content of CNTYMEs, which would provide more adsorption sites for dopamine. The differences in number or type of oxygen containing functional groups could also change the adsorption/desorption constants. The three fold increase in current is sufficient to make lasertreated CNTYMEs more practical for in vivo measurements. While the laser-treated CNTYMEs had a slightly lower ratio of dopamine oxidation for $50 \mathrm{~Hz}$ to $10 \mathrm{~Hz}$ in vivo (Fig. 2.5B) compared to in vitro (Fig. 2.4B), implantation in tissue and adherence of proteins could affect both the surface roughness and surface oxide groups. Nevertheless, high frequency measurements were achieved with only a small drop in current for laser-treated CNTYMEs in vivo. Future experiments could explore even higher scan rates, like $2000 \mathrm{~V} / \mathrm{s}$, which would permit a scan repetition frequency of $500 \mathrm{~Hz}$. Laser-treated CNTYMEs have high currents and a frequency independent response that make them promising for future studies of dopamine on the millisecond time scale. 


\subsection{Conclusions}

Laser treatment is a simple, reproducible, and efficient approach to improve the sensitivity of CNTYMEs at high scan repetition frequencies. Laser treatment increased surface area and oxygen containing functional groups on the surface, providing more adsorption sites for dopamine. In addition, the rough surface causes the oxidation signal to be scan repetition frequency independent by trapping the analytes and amplifying the signal. CNTYMEs were applied for in vivo measurement for the first time, and laser-treated CNTYMEs maintained high dopamine sensitivity with a scan repetition frequency of $50 \mathrm{~Hz}$, which is five-fold faster than the frequency conventionally applied. Laser-treated CNTYMEs are easy to fabricate, have high reproducibility and facilitate rapid measurements of dopamine with high sensitivity so they are expected to be a promising alternative to CFMEs in the future. 
Yang $\mid 100$

\subsection{References}

(1) Yang, C.; Denno, M. E.; Pyakurel, P.; Venton, B. J. Recent Trends in Carbon NanomaterialBased Electrochemical Sensors for Biomolecules: A Review. Anal. Chim. Acta 2015, 887, 17-37.

(2) Swamy, B. E. K.; Venton, B. J. Carbon Nanotube-Modified Microelectrodes for Simultaneous Detection of Dopamine and Serotonin in Vivo. Analyst 2007, 132 (9), 876884.

(3) Takmakov, P.; Zachek, M. K.; Keithley, R. B.; Walsh, P. L.; Donley, C.; McCarty, G. S.; Wightman, R. M. Carbon Microelectrodes with a Renewable Surface. Anal. Chem. 2010, 82 (5), 2020-2028.

(4) Xiao, N.; Venton, B. Rapid, Sensitive Detection of Neurotransmitters at Microelectrodes Modified with Self-Assembled SWCNT Forests. Anal. Chem. 2012, 84 (18), 7816-7822.

(5) Xiang, L.; Yu, P.; Hao, J.; Zhang, M.; Zhu, L.; Dai, L.; Mao, L. Vertically Aligned Carbon Nanotube-Sheathed Carbon Fibers as Pristine Microelectrodes for Selective Monitoring of Ascorbate in Vivo. Anal. Chem. 2014, 86 (8), 3909-3914.

(6) Xiang, L.; Yu, P.; Zhang, M.; Hao, J.; Wang, Y.; Zhu, L.; Dai, L.; Mao, L. Platinized Aligned Carbon Nanotube-Sheathed Carbon Fiber Microelectrodes for in Vivo Amperometric Monitoring of Oxygen. Anal. Chem. 2014, 86 (10), 5017-5023.

(7) Xiao, N.; Venton, B. J. Rapid, Sensitive Detection of Neurotransmitters at Microelectrodes Modified with Self-Assembled SWCNT Forests. Anal. Chem. 2012, 84 (18), 7816-7822.

(8) Yang, C.; Jacobs, C. B.; Nguyen, M. D.; Ganesana, M.; Zestos, A. G.; Ivanov, I. N.; Puretzky, A. A.; Rouleau, C. M.; Geohegan, D. B.; Venton, B. J. Carbon Nanotubes Grown on Metal Microelectrodes for the Detection of Dopamine. Anal. Chem. 2016, 88 (1), 645652.

(9) Jacobs, C. B.; Ivanov, I. N.; Nguyen, M. D.; Zestos, A. G.; Venton, B. J. High Temporal Resolution Measurements of Dopamine with Carbon Nanotube Yarn Microelectrodes. Anal. Chem. 2014, 86 (12), 5721-5727.

(10) Zestos, A. G.; Jacobs, C. B.; Trikantzopoulos, E.; Ross, A. E.; Venton, B. J. Polyethylenimine Carbon Nanotube Fiber Electrodes for Enhanced Detection of Neurotransmitters. Anal. Chem. 2014, 86, 8568-8575.

(11) Schmidt, A. C.; Wang, X.; Zhu, Y.; Sombers, L. a. Carbon Nanotube Yarn Electrodes for Enhanced Detection of Neurotransmitter Dynamics in Live Brain Tissue. ACS Nano 2013, 7864-7873.

(12) Jacobs, C. B.; Vickrey, T. L.; Venton, B. J. Functional Groups Modulate the Sensitivity and Electron Transfer Kinetics of Neurochemicals at Carbon Nanotube Modified Microelectrodes. Analyst 2011, 136 (17), 3557.

(13) Li, Z.; Liu, Z.; Sun, H.; Gao, C. Superstructured Assembly of Nanocarbons: Fullerenes, Nanotubes, and Graphene. Chem. Rev. 2015, 115 (15), 7046-7117.

(14) Vigolo, Brigitte; Penicaud, Alain; Coulon, Claude; Sauder, Cedric; Pailler, Rene; Journet, Catherine; Bernier, Patrick; Poulin, P. Macroscopic Fibers and Ribbons of Oriented Carbon Nanotubes. Science. 2000, 290 (5495), 1331-1334.

(15) Harreither, W.; Trouillon, R.; Poulin, P.; Neri, W.; Ewing, A. G.; Safina, G. Carbon Nanotube 
Fiber Microelectrodes Show a Higher Resistance to Dopamine Fouling. Anal. Chem. 2013, 85 (15), 7447-7453.

(16) Mayhew, E.; Prakash, V. Thermal Conductivity of High Performance Carbon Nanotube Yarn-like Fibers. J. Appl. Phys. 2014, 115 (17), 174306.

(17) Jiang, K.; Li, Q.; Fan, S. Nanotechnology: Spinning Continuous Carbon Nanotube Yarns. Nature 2002, 419 (6909), 801.

(18) Robinson, D. L.; Hermans, A.; Seipel, A. T.; Wightman, R. M. Monitoring Rapid Chemical Communication in the Brain. Chem. Rev. 2008, 108 (7), 2554-2584.

(19) Nguyen, M. D.; Lee, S. T.; Ross, A. E.; Ryals, M.; Choudhry, V. I.; Venton, B. J. Characterization of Spontaneous, Transient Adenosine Release in the Caudate-Putamen and Prefrontal Cortex. PLoS One 2014, 9 (1), e87165.

(20) Xu, Y.; Venton, B. J. Rapid Determination of Adenosine Deaminase Kinetics Using FastScan Cyclic Voltammetry. Phys. Chem. Chem. Phys. 2010, 12 (34), 10027-10032.

(21) Robinson, D. L.; Venton, B. J.; Heien, M. L. a V; Wightman, R. M. Detecting Subsecond Dopamine Release with Fast-Scan Cyclic Voltammetry in Vivo. Clin. Chem. 2003, 49 (10), 1763-1773.

(22) Bath, B. D.; Michael, D. J.; Trafton, B. J.; Joseph, J. D.; Runnels, P. L.; Wightman, R. M. Subsecond Adsorption and Desorption of Dopamine at Carbon-Fiber Microelectrodes. Anal. Chem. 2000, 72 (24), 5994-6002.

(23) Venton, B. J.; Troyer, K. P.; Wightman, R. M. Response Times of Carbon Fiber Microelectrodes to Dynamic Changes in Catecholamine Concentration. Anal. Chem. 2002, 74 (3), 539-546.

(24) Keithley, R. B.; Takmakov, P.; Bucher, E. S.; Belle, A. M.; Owesson-White, C. A.; Park, J.; Wightman, R. M. Higher Sensitivity Dopamine Measurements with Faster-Scan Cyclic Voltammetry. Anal. Chem. 2011, 83 (9), 3563-3571.

(25) Ross, A. E.; Venton, B. J. Sawhorse Waveform Voltammetry for Selective Detection of Adenosine, ATP, and Hydrogen Peroxide. Anal. Chem. 2014, 86 (15), 7486-7493.

(26) Ross, A. E.; Venton, B. J. Nafion-CNT Coated Carbon-Fiber Microelectrodes for Enhanced Detection of Adenosine. Analyst 2012, 137 (13), 3045-3051.

(27) Peairs, M. J.; Ross, A. E.; Venton, B. J. Comparison of Nafion- and Overoxidized Polypyrrole-Carbon Nanotube Electrodes for Neurotransmitter Detection. Anal. Methods 2011, 3 (10), 2379.

(28) Xu, G.; Li, B.; Cui, X. T.; Ling, L.; Luo, X. Electrodeposited Conducting Polymer PEDOT Doped with Pure Carbon Nanotubes for the Detection of Dopamine in the Presence of Ascorbic Acid. Sensors Actuators B Chem. 2013, 188 (2013), 405-410.

(29) Gu, L.; Jiang, X.; Liang, Y.; Zhou, T.; Shi, G. Double Recognition of Dopamine Based on a Boronic Acid Functionalized Poly(aniline-Co-Anthranilic Acid)-Molecularly Imprinted Polymer Composite. Analyst 2013, 138 (18), 5461-5469.

(30) Liu, B.; Lian, H. T.; Yin, J. F.; Sun, X. Y. Dopamine Molecularly Imprinted Electrochemical Sensor Based on Graphene-chitosan Composite. Electrochim. Acta 2012, 75 (2012), 108114.

(31) Strein, T. G.; Ewing, A. G. In Situ Laser Activation of Carbon Fiber Microdisk Electrodes. 
Anal. Chem. 1991, 63, 194-198.

(32) Strein, T. G.; Ewing, a G. Laser Activation of Microdisk Electrodes Examined by Fast-Scan Rate Voltammetry and Digital-Simulation. Anal. Chem. 1994, 66 (28), 3864-3872.

(33) McCreery, R. L. Advanced Carbon Electrode Materials for Molecular Electrochemistry. Chem. Rev. 2008, 108 (7), 2646-2687.

(34) Zachek, M. K.; Takmakov, P.; Park, J.; Wightman, R. M.; McCarty, G. S. Simultaneous Monitoring of Dopamine Concentration at Spatially Different Brain Locations in Vivo. Biosens. Bioelectron. 2010, 25 (5), 1179-1185.

(35) Phillips, P. E. M.; Wightman, R. M. Critical Guidelines for Validation of the Selectivity of inVivo Chemical Microsensors. TrAC Trends Anal. Chem. 2003, 22 (8), 509-514.

(36) Williams, J. E.; Wieczorek, W.; Willner, P.; Kruk, Z. L. Parametric Analysis of the Effects of Cocaine and Cocaine Pretreatment on Dopamine Release in the Nucleus Accumbens Measured by Fast Cyclic Voltammetry. Brain Res. 1995, 678 (1-2), 225-232.

(37) Rice, M. ; Russo-Menna, I. Differential Compartmentalization of Brain Ascorbate and Glutathione between Neurons and Glia. Neuroscience 1997, 82 (4), 1213-1223.

(38) Xiang, L.; Yu, P.; Hao, J.; Zhang, M.; Zhu, L.; Dai, L.; Mao, L. Vertically Aligned Carbon Nanotube-Sheathed Carbon Fibers as Pristine Microelectrodes for Selective Monitoring of Ascorbate in Vivo. Anal. Chem. 2014, 86 (8), 3909-3914.

(39) Claxton, N. S.; Fellers, T. J.; Davidson, M. W. Microscopy, Confocal. In Encyclopedia of Medical Devices and Instrumentation; John Wiley \& Sons, Inc.: Hoboken, NJ, USA, 2006; Vol. 1979, pp 1-37.

(40) Streeter, I.; Wildgoose, G. G.; Shao, L.; Compton, R. G. Cyclic Voltammetry on Electrode Surfaces Covered with Porous Layers: An Analysis of Electron Transfer Kinetics at SingleWalled Carbon Nanotube Modified Electrodes. Sensors Actuators, B Chem. 2008, 133 (2), 462-466.

(41) Henstridge, M. C.; Compton, R. G. Mass Transport to Micro- and Nanoelectrodes and Their Arrays: A Review. Chem. Rec. 2012, 12 (1), 63-71.

(42) Aoki, K. Theory of Ultramicroelectrodes. Electroanalysis 1993, 5 (8), 627-639.

(43) Arrigan, D. W. M. Nanoelectrodes, Nanoelectrode Arrays and Their Applications. Analyst 2004, 129 (12), 1157.

(44) Filik, J.; May, P. W.; Pearce, S. R. J.; Wild, R. K.; Hallam, K. R. XPS and Laser Raman Analysis of Hydrogenated Amorphous Carbon Films. Diam. Relat. Mater. 2003, 12 (3-7), 974-978.

(45) Ivanov, I.; Puretzky, A.; Eres, G.; Wang, H.; Pan, Z.; Cui, H.; Jin, R.; Howe, J.; Geohegan, D. B. Fast and Highly Anisotropic Thermal Transport through Vertically Aligned Carbon Nanotube Arrays. Appl. Phys. Lett. 2006, 89 (22), 223110.

(46) Washer, G.; Blum, F. Raman Spectroscopy for the Nondestructive Testing of Carbon Fiber. Res. Lett. Mater. Sci. 2008, 2008, 1-3.

(47) Roberts, J. G.; Moody, B. P.; McCarty, G. S.; Sombers, L. a. Specific Oxygen-Containing Functional Groups on the Carbon Surface Underlie an Enhanced Sensitivity to Dopamine at Electrochemically Pretreated Carbon Fiber Microelectrodes. Langmuir 2010, 26 (11), 9116-9122. 
Chapter 4: $\mathrm{O}_{2}$ Plasma Etching and Anti-Static Gun Surface Modifications for CNT Yarn Microelectrode Improve Sensitivity and Anti-Fouling Properties 


\section{Abstract}

Carbon nanotube (CNT) based microelectrodes exhibit rapid, sensitive, and selective detection of neurotransmitters. However, the lack of understanding of how different carbon nanomaterials and different surface treatments limits the further improvement on electrochemical performance such as sensitivity and fouling resistance. In this work, we introduce two simple, reproducible, low-cost, and efficient surface modification methods for carbon nanotube yarn microelectrodes (CNTYMEs): $\mathrm{O}_{2}$ plasma etching and anti-static gun treatment. $\mathrm{O}_{2}$ plasma etching was performed by a microwave plasma system with oxygen gas flow and the optimized time for treatment was 1 minute. The anti-static gun treatment is a new, cheap, and simple surface modification and two triggers of the anti-static gun was the optimized number on the CNTYME surface. Dopamine sensitivity of CNTYMEs increased by three fold after $\mathrm{O}_{2}$ plasma etching and four fold after anti-static gun treatment. The two surface treatment methods provide different mechanisms to tune the surface properties: $\mathrm{O}_{2}$ plasma etching increased the sensitivity due to increased surface oxygen content but did not affect surface roughness while the increased sensitivity after anti-static gun treatment was due to the increased surface roughness but not oxygen content. The effect of tissue fouling on CNT yarns was studied for the first time, and the relatively hydrophilic surface after $\mathrm{O}_{2}$ plasma etching provide better resistance to fouling than unmodified and anti-static gun treated CNTYMEs. Overall, $\mathrm{O}_{2}$ plasma etching and anti-static gun treatment improve sensitivity of CNTYMEs by different mechanisms, providing the possibility to tune the CNTYME surface for different in vivo neurotransmitter measurements. 


\subsection{Introduction}

CNT yarns are macrostructured carbon materials directly spun from CNTs arrays., ${ }^{1,2}$ Schmidt et al. first used CNT yarn microelectrodes (CNTYMEs) for neurotransmitter monitoring with improved selectivity, sensitivity, spatial resolution, and faster electron transfer kinetics compared to the conventionally used carbon fiber microelectrodes (CFMEs). ${ }^{3}$ In comparison to randomly distributed and tangled CNTs produced by dip coating, well-aligned CNT yarns have the advantages of high electroactivity, chemical stability and conductivity, controllable size, and promising anti-fouling properties. ${ }^{4-6}$ Oxygen containing functional groups, such as quinones, hydroxyl, and carboxylic acids are known to be negatively charged at physiological $\mathrm{pH}$ and have electrostatic interactions with positively charged neurotransmitters such as dopamine $;{ }^{7}$ CNTYMEs surface has intrinsically abundant oxygen containing functional groups which provide abundant adsorption sites for dopamine. ${ }^{8}$ Moreover, fabricating CNT yarns directly into electrodes in a manner similar to CFMEs dramatically simplifies the electrode fabrication process and improves the reproducibility. ${ }^{9}$ Our group found the dopamine signal is independent to the repetition rate applied on fast scan cyclic voltammetry (FSCV) at CNTYMEs, an advantage over conventionally used CFMEs which lose sensitivity with increasing repetition rate, enabling CNTYMEs for the rapid and sensitive neurotransmitters detection. ${ }^{8}$ Since CNT yarns are a soft material, cutting it with scalpel, which is used for carbon fibers, will not work: the end of the yarn will puff out after cutting so the geometry and homogeneity are changed. Therefore, all the applications of CNT yarns are limited to disk electrodes. The disk geometry, however, restricts the overall surface area and thus limits the sensitivity. Therefore, the sensitivity and fouling resistance need to be further improved due to the significant fouling when CNTYMEs are placed in vivo.

Carbon electrode sensitivity can be improved by several surface modification methods. Acid treatment ${ }^{10}$, electrochemical activation ${ }^{11}$, and spark etching ${ }^{12}$ change the surface properties. Polymer coating ${ }^{13-15}$ and metal nanoparticle coating ${ }^{16,17}$ improves sensitivity but he slower electron transfer rate caused by coatings and the complicated modification methods limit their 
reproducibility and further applications. Recently, we have reported laser treatment as a solventfree, reproducible, and efficient approach to further improve the sensitivity of CNTYMEs, and applied the modified CNTYMEs for rapid in vivo neurotransmitters detection for the first time. ${ }^{18}$ Similar to the previous work on the laser treated CFMEs laser activation ${ }^{19,20}$, the enhanced sensitivity of CNTYMEs after laser treatment is due to the synergistic effect of both larger surface roughness and larger amount of oxygen-containing functional groups created by laser pulses. In comparison, the sensitivity improvement on CNTYMEs is greater than that at CFMEs, which would be caused by the dramatically increased surface roughness mainly from the nanostructure on CNT yarn surface. ${ }^{18}$ The laser etching, however, requires an optical system set-up and expensive laser exciter. Surface treatments which are more accessible for general neuroscience labs would be advisable.

In this work, two surface modification methods for CNT yarn microelectrodes were evaluated and optimized: $\mathrm{O}_{2}$ plasma etching and anti-static gun treatment. These surface treatment methods are lower cost, simpler, and more feasible than methods such as laser treatment. $\mathrm{O}_{2}$ plasma etching has been widely used for surface cleaning and providing surface oxide groups. ${ }^{21-23}$. Dopamine detection sensitivity can be improved at several carbon nanomaterials with $\mathrm{O}_{2}$ plasma etching, ${ }^{24-27}$ but the application on CNT yarns has not been evaluated. $\mathrm{O}_{2}$ plasma etching on CNTYMEs is performed by a microwave plasma system with oxygen gas flow and dopamine sensitivity is improved by more than three-fold. Anti-static gun treatment also significantly improves sensitivity and is applied as a novel nanomaterial surface modification method for the first time. Anti-static gun treatment is simply performed by applying several triggers of anti-static gun on CNT yarn surface, which flows ions across the surface. Antistatic gun treatment increased the current at the CNTYME by four-fold but did not change the current at a carbon fiber because it only changes the surface roughness and alignment of the nanomaterial. Notably, $\mathrm{O}_{2}$ plasma etching and anti-static gun treatment specifically increase the amount of oxygen content and surface roughness, respectively, without changing another one, 
which provide promising platforms for the study on how surface roughness and oxide groups influence the electrochemistry. In vivo detection of neurotransmitters is challenging because fouling caused by macro biomolecules could severely reduce sensitivity and electron transfer rate. ${ }^{6,28}$ The relatively hydrophilic surface after $\mathrm{O}_{2}$ plasma etching maintained more dopamine signal after tissue exposure than unmodified and anti-static gun treated CNTYMEs due to its large amount of oxygen-containing functional groups. Overall, the $\mathrm{O}_{2}$ plasma etching and anti-static gun treatments are simple, low-cost, and efficient surface treatments for CNTYMEs, and provide different mechanisms to tune the surface properties to increase sensitivity and reduce fouling. 


\subsection{Experimental Section}

\subsubsection{Carbon Nanotube Yarn Microelectrode Preparation}

A $0.68 \mathrm{~mm}$ ID $\times 1.2 \mathrm{~mm}$ OD (A-M Systems, Carlsborg, WA) glass capillary was pulled into a glass pipet puller and cut to have an opening diameter of $\sim 50 \mu \mathrm{m}$. A piece of commercially available CNTY (10-25 $\mu \mathrm{m}$ in diameter, $1-2 \mathrm{~cm}$ long, General Nano, LLC, Cincinnati, OH) was inserted into the glass pipette and epoxied in place with Epon Resin 828 (Miller-Stephenson, Danbury, CT) mixed with 14\% (w/w) 1,3-phenylenediamine hardener (Sigma-Aldrich, St. Louis, $\mathrm{MO}$ ) heated to $85^{\circ} \mathrm{C}$. The epoxied electrodes were cured overnight at room temperature and then heated at $100^{\circ} \mathrm{C}$ for $2 \mathrm{~h}$ and at $150^{\circ} \mathrm{C}$ overnight. Electrodes were polished at a $45^{\circ}$ angle on a fine diamond abrasive plate (Sutter Instruments model BV-10, Novato, CA) to create an elliptical active area. Cylindrical CFMEs were fabricated using 7- $\mu$ m-diameter T-650 carbon fibers (Cytec Technologies, Woodland Park, NJ). ${ }^{8}$

\subsection{2 $\mathrm{O}_{2}$ Plasma Treatment}

The polished disk CNT yarn microelectrodes were oxygen plasma etched using PVATePla Microwave Plasma System 400 H2 (PVA TEPLA, Corona, CA). About 20 - 30 CNTYMEs fixed on silicon chip were placed in a plasma generator and etched with ignite plasma power of $600 \mathrm{~W}$, with the etching duration of 1 or 3 minutes at room temperature. The oxygen gas flow rate was 250 sccm to achieve a chamber oxygen pressure of about 970 mTorr.

\subsubsection{Anti-static gun Treatment}

Anti-static treatment was applied on CNT yarn microelectrode by simply trigger anti-static gun for several times (1, 2 or 4 triggers). Milty Zerostat 3 anti-static gun (Milty Co., UK) was used with unit cost less than 100 US dollars. The anti-static gun incorporates a unique Piezo Crystal device which generates a positive electrical stream of lons with a gentle slow squeeze of the 
trigger, and a negative stream of ions upon slow release of the trigger with about 1.5 Coulombs. The tip of CNT yarn microelectrodes were placed approximately $5 \mathrm{~cm}$ away from the muzzle. A trigger was defined as squeezing the trigger slowly for about two seconds, followed by a slow release for another two seconds. The anti-static gun treated CNTYMEs were left in the air for about 1 hour equilibration before electrochemical measurements.

\subsubsection{Solutions}

Dopamine hydrochloride and ascorbic acid were purchased from Sigma-Aldrich (St. Louis, MO). A $10 \mathrm{mM}$ stock solution was prepared in $\mathrm{HClO}_{4}$, and were diluted daily to the desired concentration in phosphate buffered saline $\left(131.3 \mathrm{mM} \mathrm{NaCl}, 3.00 \mathrm{mM} \mathrm{KCl}, 10 \mathrm{mM} \mathrm{NaH}_{2} \mathrm{PO}_{4}, 1.2\right.$ $\mathrm{mM} \mathrm{MgCl}_{2}, 2.0 \mathrm{mM} \mathrm{Na}_{2} \mathrm{SO}_{4}$, and $1.2 \mathrm{mM} \mathrm{CaCl}_{2}$ with the $\mathrm{pH}$ adjusted to 7.4$)$.

\subsubsection{Electrochemistry}

FSCV was performed with a ChemClamp potentiometer (Dagan, Minneapolis, MN, with 1 MOhm Headstage). The waveform was generated and the data was collected using a High Definition Cyclic Voltammetry (HDCV) breakout box, HDCV analysis software program (UNC Chemistry Department, Electronics Design Facility) and PCle-6363 computer interface cards (National Instruments, Austin, TX). Electrodes were backfilled with $1 \mathrm{M} \mathrm{KCl}$ and a silver wire was inserted to connect the electrode to the potentiostat headstage. The typical triangular waveform swept the applied potential from $-0.4 \mathrm{~V}$ to $1.3 \mathrm{~V}$ at $400 \mathrm{~V} / \mathrm{s}$ versus an $\mathrm{Ag} / \mathrm{AgCl}$ reference electrode, at a scan repetition frequency of $10 \mathrm{~Hz}$. The repetition rate was varied for some experiments.

Electrodes were tested using a flow-injection system, as previously described. Analyte was injected for 5 seconds and current versus time traces were obtained by integrating the current in a $100 \mathrm{mV}$ window centered at the oxidation peak for each cyclic voltammogram (CV). Background-subtracted CVs were calculated by subtracting the average of 10 background scans, 
taken before the compound was injected, from the average of five CVs recorded after the analyte bolus was injected.

\subsubsection{Surface Characterization}

Scanning electron microscope (SEM) images were taken on Merlin field emission SEM (Zeiss, Thornwood, NY) with a secondary electron detector using an accelerating voltage of $2 \mathrm{kV}$ and a working distance of $5.0 \mathrm{~mm}$. The Energy-dispersive X-ray spectroscopy (EDS) data were acquired for 300 seconds on electrode surface with an acceleration voltage of $5 \mathrm{kV}$ at a working distance of $5.0 \mathrm{~mm}$. Raman spectroscopy measurements were performed with a Renishaw 100 confocal micro-Raman system (Renishaw, Hoffman Estates, IL) with a 1800 lines/mm diffraction grating, $532 \mathrm{~nm}$ laser focused to a spot size of about $2 \mu \mathrm{m}$ through a 100x objective, and a Peltiercooled charge-coupled device detector. Three-dimensional laser (violet laser, $408 \mathrm{~nm}$ ) scanning confocal microscopy (VK-X, Keyence, IL) was performed to measure the surface roughness.

\subsubsection{Statistics}

All values are given as mean \pm standard error of the mean (SEM) for $n$ number of electrodes and all error bars are SEM. Paired or unpaired t tests were performed to compare properties between two groups. A one-way ANOVA with Bonferonni post-tests was used to compare effects among multiple groups. All statistics were performed in GraphPad Prism6 (GraphPad Software,Inc., La Jolla, CA). Scanning electron microscopy images were processed using ImageJ (Rasband, W.S., National Institutes of Health, Bethesda, MD,).

\subsubsection{In Tissue Fouling}

Male Sprague-Dawley rats (250-350 g) purchased from Charles River were housed in a vivarium and given food and water ab libitum. All experiments were approved by the Animal Care 
and Use Committee of the University of Virginia. The brains for homogenate fouling studies were obtained after in vivo experiments, in which the rat was anesthetized with urethane $(1.5 \mathrm{mg} / \mathrm{kg}$ i.p.), the scalp shaved, and $0.25 \mathrm{~mL}$ bupivicaine ( $0.25 \%$ solution) given subcutaneously. Brains were rapidly removed from the skulls for usage. In place of evaluating each electrode in a living animal, the use of brain tissue homogenates enabled fouling experiments to be carried out on many electrodes using a comparatively small number of animals. The microelectrodes were precalibrated for their response to $1 \mu \mathrm{M}$ dopamine and then placed in the tissue sample for two hours without potential cycling. After immersing in PBS solution for 15 minutes, their responses to $1 \mu \mathrm{M}$ dopamine were tested and compared. 


\subsection{Results and Discussion}

\subsubsection{Parameter Optimization}

$\mathrm{O}_{2}$ plasma etched CNTYMEs were treated by a microwave plasma system with a power of $600 \mathrm{~W}$ and oxygen gas flow rate of $250 \mathrm{sccm}$ for either 1 or $3 \mathrm{~min}$. The response to $1 \mu \mathrm{M}$ dopamine was characterized using FSCV on the same CNTYMEs before/after 1 min or 3 min treatment (Figure 4.1). Both the oxidation and reduction currents for $1 \mu \mathrm{M}$ dopamine increased about three fold after the treatments, and the improvement is larger at CNTYMEs with 3 min $\mathrm{O}_{2}$ plasma etching than with 1 min etching. However, after 3 min of $\mathrm{O}_{2}$ plasma etching, the separation between the oxidation and reduction peak potentials $\left(\Delta \mathrm{E}_{\mathrm{p}}\right)$ is larger than that that at 1 min etched CNTYME, indicating that the long duration etching slows the electron transfer rate. The temporal response at CNTYMEs after 1 min etching did not change compared to pretreatment, but the temporal response after 3 mins etching is slower, as the current increased slowly and did not go back to baseline (Figure 4.1E). Although 3 mins $\mathrm{O}_{2}$ plasma etched CNTYMEs has a higher oxidation current improvement ratio of $3.2 \pm 0.6(n=5)$ at the same electrodes, the longer duration of etching also causes more noise, making the 3 mins $\mathrm{O}_{2}$ plasma etched CNTYMEs have a worse limit of detection (LOD) $(26 \pm 9 \mathrm{nM}, \mathrm{n}=5)$ compared to 1 min $\mathrm{O}_{2}$ plasma etching $(11 \pm 2 \mathrm{nM}, \mathrm{n}=$ 6, unpaired t-test, $p \leq 0.05)$. 


\section{$\mathrm{O}_{2}$ Plasma Etched CNTYMEs (1 min)}
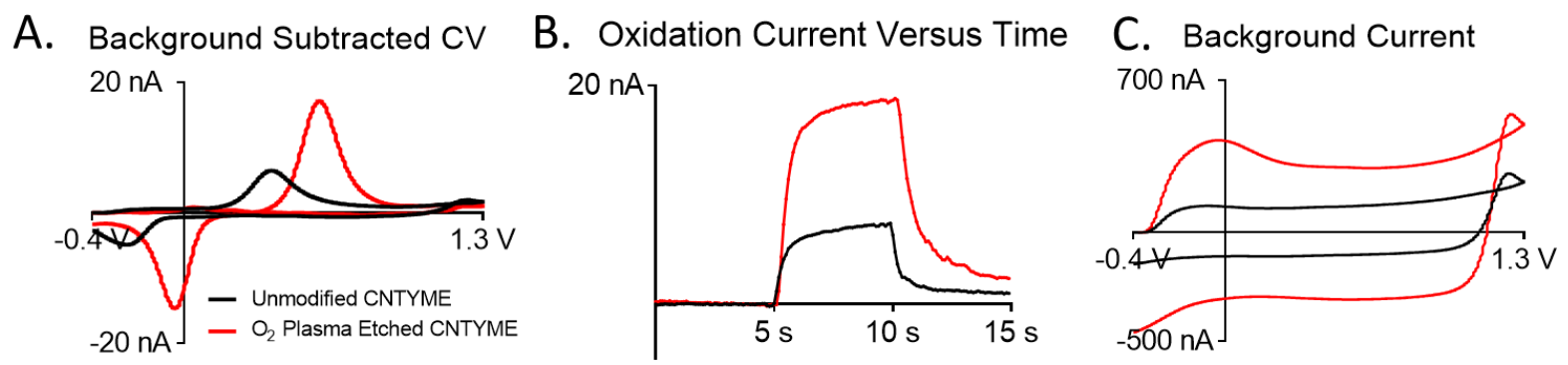

\section{$\mathrm{O}_{2}$ Plasma Etched CNTYMEs (3 mins)}
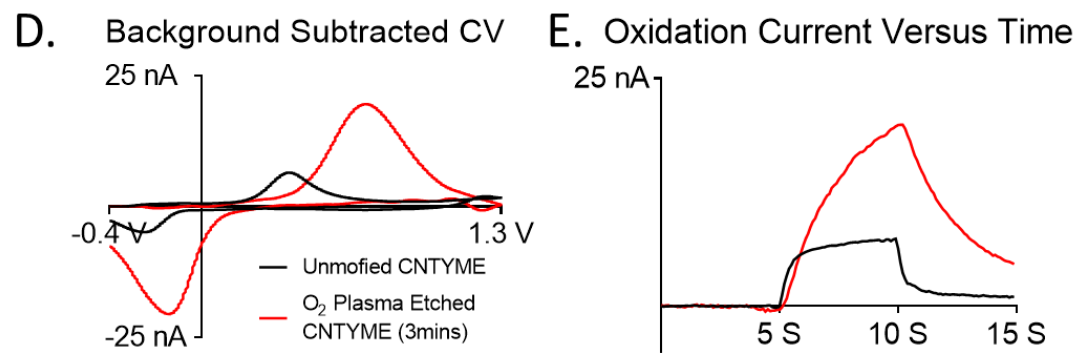

F. Background Current
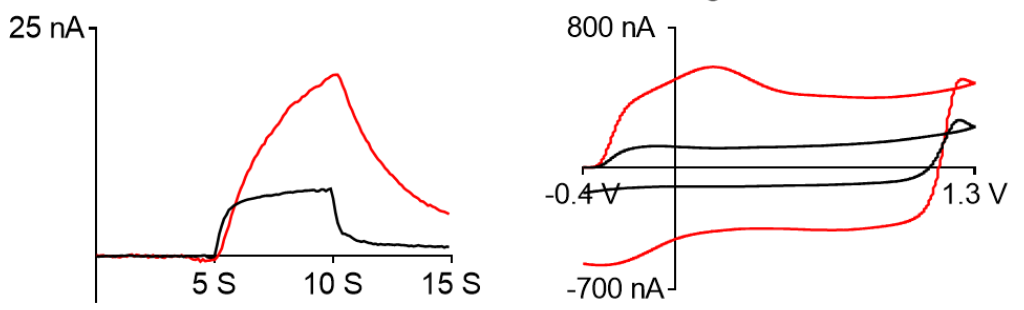

Figure 4.1: Comparison of the response to $1 \mu \mathrm{M}$ dopamine at an unmodified disk CNTYME (black line) and the same electrode after treatment (red line) of 1 min (A-C) and 3 mins (D-E) $\mathrm{O}_{2}$ plasma etching. Electrochemical response was measured with scan rate of $400 \mathrm{~V} / \mathrm{s}$ and scan repetition frequency of $10 \mathrm{~Hz}$. (A, D) Background subtracted cyclic voltammograms to $1 \mu \mathrm{M}$ dopamine, (B, E) measured oxidation current versus time for a flow injection analysis experiment, and (C, F) background currents in PBS buffer.

Figure 4.2 shows the scanning electron microscopy (SEM) images on the same CNTYMEs after $1 \mathrm{~min}$ or $3 \mathrm{~min}$ treatment. Compared to the mild modification of $1 \mathrm{~min} \mathrm{O}_{2}$ plasma etching that did not cause obvious morphology changes, the CNT yarn surface morphology after 3 mins $\mathrm{O}_{2}$ plasma etching is significantly changed. Large amount of broken CNT bundles were formed (Figure 4.2A), which is likely to cause the reduced electron transfer rate and slower temporal response. Therefore, $1 \mathrm{~min} \mathrm{O}_{2}$ plasma etching was chosen as optimal for further electrochemical and physical characterizations. 

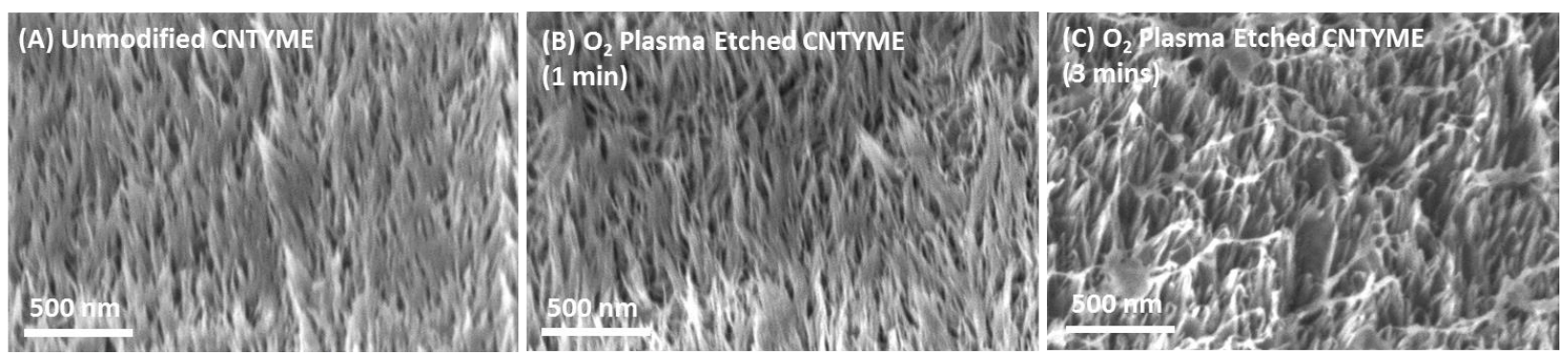

Figure 4.2: SEM images of (A) an unmodified CNTYME and the same electrode after (B) 1 min and (C) 3 mins $\mathrm{O}_{2}$ plasma etching. Scale bar: $500 \mathrm{~nm}$.

Anti-static gun treatment is a novel surface treatment for electrodes and is simply performed by applying triggers of anti-static gun with the muzzle about $5 \mathrm{~cm}$ away from the microelectrode tip. The piezo crystal device in the anti-static gun generates a charged electrical air stream. To optimize the amount of triggers, CNTYMEs were treated with 1, 2, or 4 triggers. Figure 4.3 shows the example CVs and the oxidation and reduction currents for $1 \mu \mathrm{M}$ dopamine increased about two folds after 1 and 4 triggers, which is smaller than the four-fold signal increase at CNTYMEs with 2 triggers. Statistics from multiple electrodes are shown in Table 4.1; CNTYMEs with 2 triggers give the largest signal improvement and best LOD compared to 1 and 4 triggers. CNTYMEs after treatments give about two fold increase in background current. The temporal response does not change much after 1 and 2 triggers anti-static gun treatment, but it slows much after treatment with 4 triggers. SEM images taken at a CNTYME with 1 trigger (Figure 4.4) do not show much difference compared with unmodified electrodes, which explains the lower signal enhancement. In comparison, the CNT bundles becomes straight up after 2 triggers treatment. However, surface morphology at CNTYMEs with 4 triggers changed significantly compared to unmodified electrode and even electrode with 2 triggers: a secondary structure of CNT bundles is observed, which introduces noise, increase the LOD, and slows the temporal response. Therefore, CNTYMEs with 2 triggers was chosen as optimal anti-static gun treatment for further electrochemical characterization. 
Table 4.1: Comparison of dopamine detection at anti-static gun treated CNTYMEs with different triggers

\begin{tabular}{|c|c|c|c|c|}
\hline & $\begin{array}{c}\text { Oxidation } \\
\text { Current Ratio }\end{array}$ & $\begin{array}{c}\text { Background } \\
\text { Current Ratio }\end{array}$ & $\Delta \mathrm{E}_{\mathrm{p}}(\mathrm{V})$ & $\mathrm{LOD} / \mathrm{nM}$ \\
\hline $\begin{array}{c}\text { CNTYMEs with 1 trigger } \\
(\mathrm{n}=5)\end{array}$ & $1.5 \pm 0.1$ & $1.6 \pm 0.1$ & $0.65 \pm 0.02$ & $21 \pm 4$ \\
\hline $\begin{array}{c}\text { CNTYMEs with 2 triggers } \\
(\mathrm{n}=5)\end{array}$ & $4.2 \pm 0.5$ & $1.9 \pm 0.2$ & $\begin{array}{c}0.612 \pm \\
0.006\end{array}$ & $12 \pm 2$ \\
\hline $\begin{array}{c}\text { CNTYMEs with 4 triggers } \\
(\mathrm{n}=5)\end{array}$ & $2.6 \pm 0.4$ & $1.8 \pm 0.2$ & $\begin{array}{c}0.607 \pm \\
0.004\end{array}$ & $24 \pm 1$ \\
\hline
\end{tabular}

\section{Anti-Static Gun Treated CNTYMEs (1 Trigger)}

A. Background Substracted CV

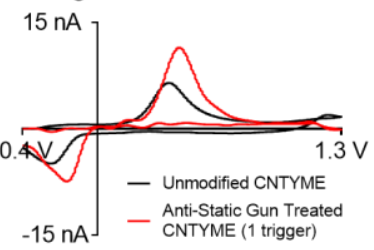

B. Oxidation Current Versus Time

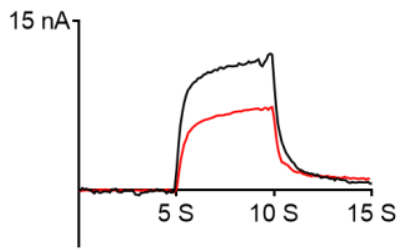

C. Background Current

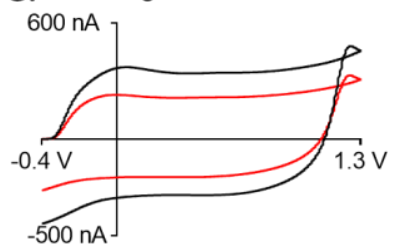

Anti-Static Gun Treated CNTYMEs (2 Triggers)

D. Background Subtracted CV

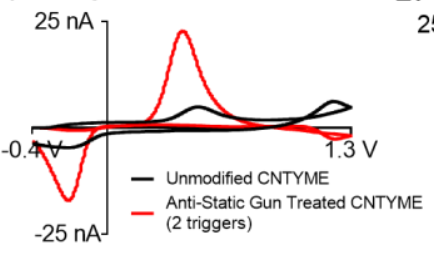

E. Oxidation Current Versus Time $25 \mathrm{nA}$

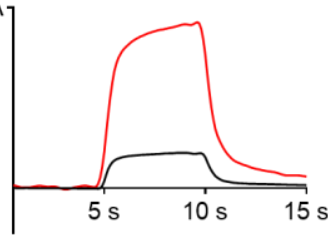

F. Background Current

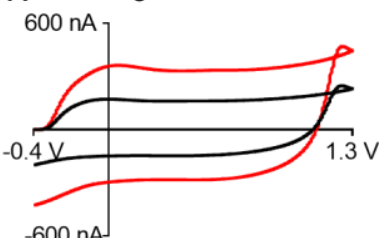

\section{Anti-Static Gun Treated CNTYMEs (4 Triggers)}

G. Background Substracted CV

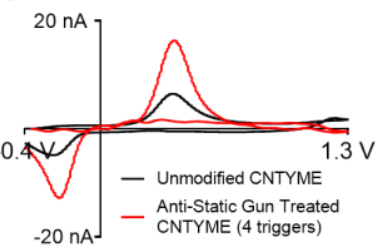

H. Oxidation Current Versus Time $20 \mathrm{nA}$

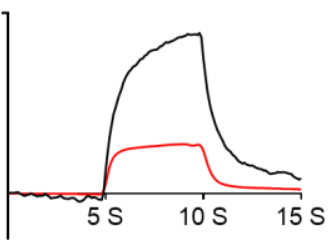

I. Background Current

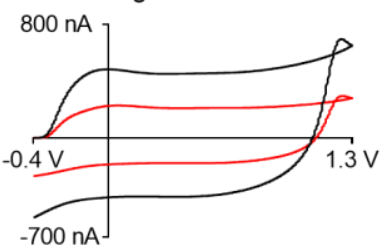

Figure 4.3: Comparison of the response to $1 \mu \mathrm{M}$ dopamine at an unmodified disk CNTYME (black line) and the same electrode after treatment (red line) of 1 trigger (A-C), 2 triggers (D-E), and 4 triggers of anti-static gun modifications. Electrochemical response was measured with scan rate of $400 \mathrm{~V} / \mathrm{s}$ and scan repetition frequency of $10 \mathrm{~Hz}$. (A, D, G) Background subtracted cyclic voltammograms to $1 \mu \mathrm{M}$ dopamine, $(B, E, H)$ measured oxidation current versus time for a flow injection analysis experiment, and $(\mathrm{C}, \mathrm{F}, \mathrm{I})$ background currents in PBS buffer. 

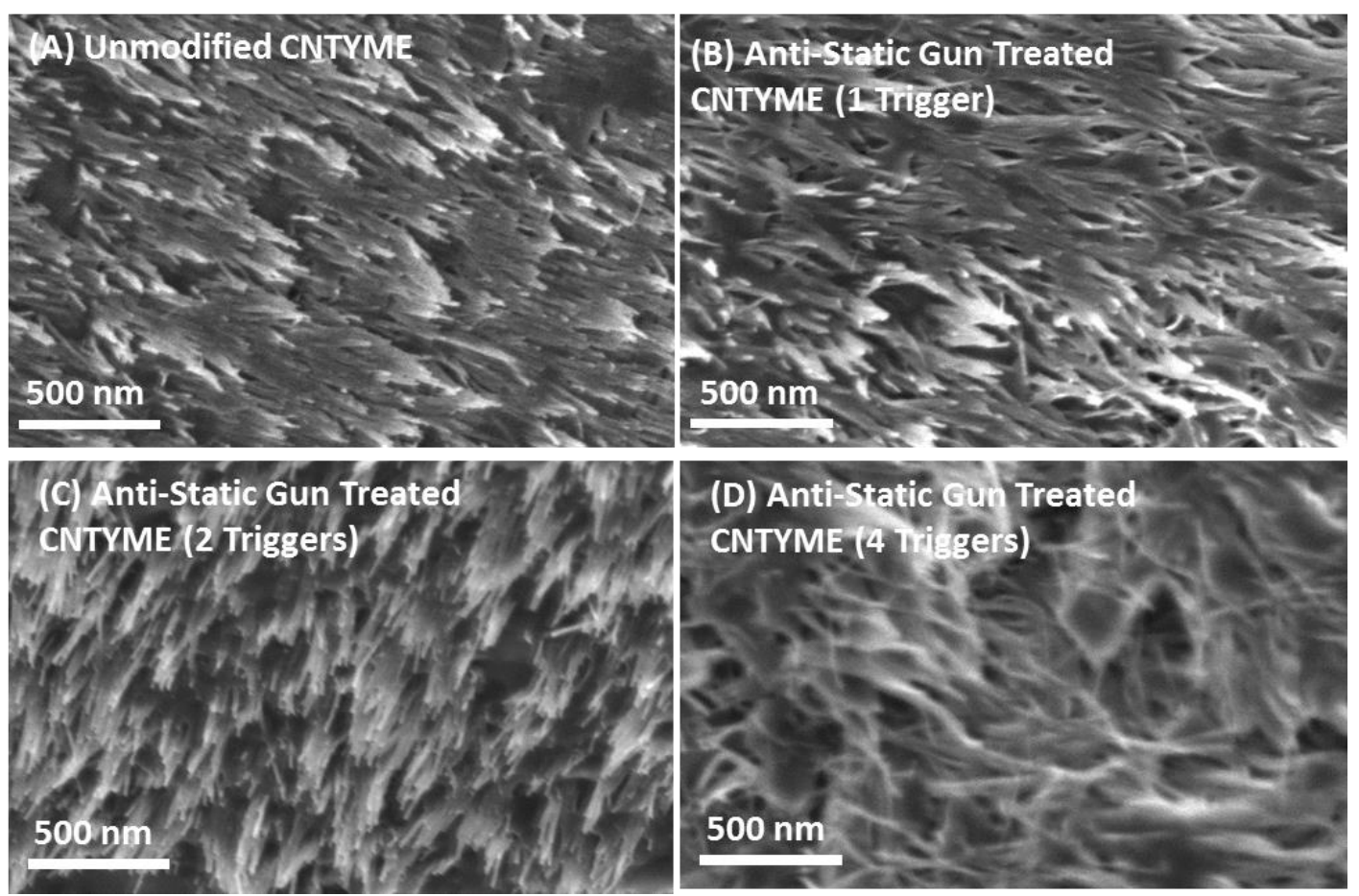

Figure 4.4: SEM images of $(A)$ an unmodified CNTYME and the same electrode after anti-static gun treatments with (B) 1 trigger, (C) 2 triggers, and (D) 4 triggers. Scale bar: $500 \mathrm{~nm}$.

We also tested the effect of time after 2 triggers of the antistatic gun on measured current. Figure 4.5 shows the oxidation current for $1 \mu \mathrm{M}$ dopamine at unmodified CNTYMEs and the same electrodes with 2 triggers modifications, immediately after modification or after 1 to 5 hours equilibration in air. The signal reaches equilibrium after 1 hour and does not change significantly up to five hours (one-way ANOVA, Bartlett's test, $p>0.5, n=4$ ): any extra charge on CNT yarn surface might be balanced in air after $1 \mathrm{H}$. Thus, all electrochemical characterizations were performed after 1 hour equilibration once electrodes were modified. 


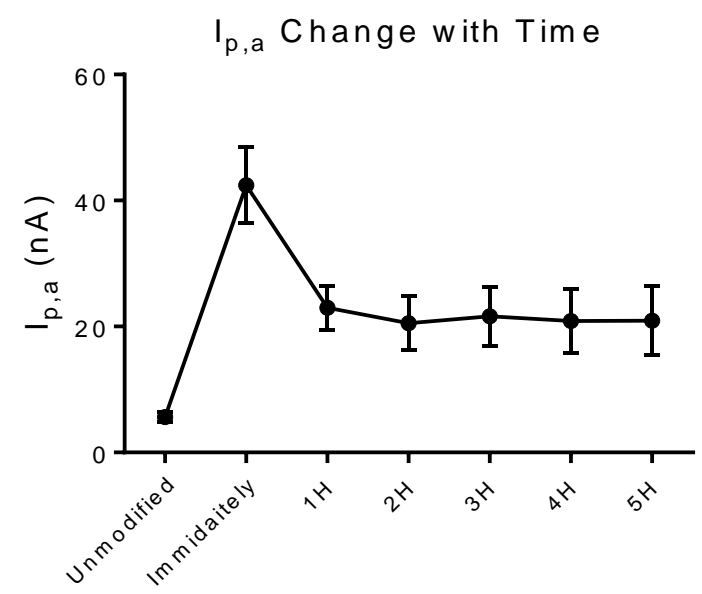

Figure 4.5: Oxidation current $\left(I_{p, a}\right)$ change with time at anti-static gun treated CNTYMEs with 2 triggers. (A) $I_{p, a}$ to $1 \mu \mathrm{M}$ dopamine at unmodified CNTYME, immediately measured after 2 triggers anti-static gun treatment modification, and measured after 1-5 hours equilibration in air after antistatic gun treatment on the same electrodes. The measurement taken right after anti-static gun treatment shows significantly larger $I_{p, a}$ than measurements taken after up to 5 hours equilibration in air (one-way ANOVA, Bartlett's test, $p \leq 0.01, n=4$ ). Error bar is standard error of mean.

Finally, the 5 hour short-term stability and 24 hour stability on CNTYMEs with 1 min $\mathrm{O}_{2}$ plasma etching and 2 triggers anti-static gun treatment were tested. To test short-term stability, electrochemical tests were performed over 5 hours with potential cycling and the relative standard deviations (RSD) were 4.08\% $(n=5)$ and 5.76\% $(n=5)$ for $\mathrm{O}_{2}$ plasma etched and anti-static gun treated CNTYMEs, respectively. In addition, electrodes had promising shelf stability as testing an electrode once and again after 24 hours storage shows RSDs of $3.4 \%$ and $2.6 \%$ for $\mathrm{O}_{2}$ plasma etched and anti-static gun treated CNTYMEs, respectively. This promising stability allows both $\mathrm{O}_{2}$ plasma etched and anti-static gun treated to be used for general in vivo neurotransmitter monitoring experiment ( 4 hours long) ${ }^{29}$ and to be made in batches.

\subsubsection{Comparison of Electrochemical Properties of $\mathrm{O}_{2}$ plasma etched and Anti-Static Gun Treated}

\section{CNTYMES}

Electrochemical properties were compared for the optimized $\mathrm{O}_{2}$ plasma etched $(1 \mathrm{~min})$ and anti-static gun treated (2 triggers) CNTYMEs. The CVs for 1 uM dopamine for both treatments 
show a sensitivity increase, but there are differences with potentials shifting with the $\mathrm{O}_{2}$ plasma etched electrodes. For $\mathrm{O}_{2}$ plasma etching, both the oxidation and reduction currents for $1 \mu \mathrm{M}$ dopamine increased about three folds (Fig. 4.1A, Table 4.2). The statistics obtained from multiple electrodes shows the $4.3 \pm 0.4$ fold signal improvement for anti-static gun treatment is larger than the $3.0 \pm 0.4$ fold increase at $\mathrm{O}_{2}$ plasma etched CNTYMEs (Table 4.2\&4.3, unpaired t-test, $\mathrm{p} \leq$ $0.05)$.

Table 4.2: Comparison of dopamine detection at unmodified and $\mathrm{O}_{2}$ plasma etched CNTYMEs

\begin{tabular}{|c|c|c|c|c|}
\hline & $\begin{array}{c}\text { Oxidation } \\
\text { Current/nA }\end{array}$ & $\begin{array}{c}\text { Background } \\
\text { Current/nA }\end{array}$ & $\Delta \mathrm{E}_{\mathrm{p}}$ & LOD/nM \\
\hline Unmodified (n=6) & $5.5 \pm 0.9$ & $122 \pm 25$ & $0.67 \pm 0.02$ & $21 \pm 4$ \\
\hline $\begin{array}{c}\mathrm{O}_{2} \text { Plasma Etching (1min, } \\
\mathrm{n}=6)\end{array}$ & $16 \pm 3^{* *}$ & $298^{*} \pm 35^{* * *}$ & $\begin{array}{c}0.607 \pm \\
0.003^{*}\end{array}$ & $11 \pm 2^{*}$ \\
\hline $\begin{array}{c}\text { Treated/unmodified } \\
\text { Ratio }\end{array}$ & $3.0 \pm 0.4$ & $2.3 \pm 0.4$ & $0.91 \pm 0.02$ & $0.7 \pm 0.1$ \\
\hline
\end{tabular}

Significantly different than unmodified: ${ }^{*}$ paired $t$ test, $p \leq 0.05,{ }^{* *}$ paired $t$ test, $p \leq 0.01,{ }^{* * *}$ paired $t$ test, $p \leq 0.001$.

$\Delta \mathrm{E}_{\mathrm{p}}$ decreased after $\mathrm{O}_{2}$ plasma etching, indicating improved electron transfer rate (Fig. 4.1A, Table 4.2). ${ }^{30}$ Notably, both of the oxidation and reduction potentials shifted positively after modification. The possible reason is that $\mathrm{O}_{2}$ plasma etching provides abundant oxygen-containing functional groups, changing the surface charge. Therefore, an accumulation of positive charge is required for the deprotonation of the oxides groups, which are negatively charged under physiological $\mathrm{pH}$, to reach the point of zero charge of the electrode. ${ }^{31}$ For anti-static gun treated CNTYMEs, the current increase was similar, but the potentials did not shift positively (Fig. 4.3D, Table 4.3). The $\Delta \mathrm{E}_{\mathrm{p}}$ also decreased after anti-static gun treatment. The significantly decreased $\Delta \mathrm{E}_{\mathrm{p}}$ after anti-static gun treatment (paired t-test, $\mathrm{p} \leq 0.05, \mathrm{n}=8$ ) and improved LOD (paired t-test, 
$p \leq 0.05, n=8)$ indicate the improvement on both electron transfer rate and the signal to noise ratio.

Table 4.3: Comparison of dopamine detection at unmodified and anti-static gun treated CNTYMEs

\begin{tabular}{|c|c|c|c|c|}
\hline & $\begin{array}{c}\text { Oxidation } \\
\text { Current/nA }\end{array}$ & $\begin{array}{c}\text { Background } \\
\text { Current/nA }\end{array}$ & $\Delta \mathrm{E}_{\mathrm{p}}$ & LOD/nM \\
\hline Unmodified (n=8) & $5.2 \pm 0.3$ & $130 \pm 19$ & $0.67 \pm 0.02$ & $24 \pm 5$ \\
\hline $\begin{array}{c}\text { Anti-static gun treatment } \\
\text { (2 triggers, } \mathrm{n}=8)\end{array}$ & $22 \pm 1^{* * * *}$ & $252 \pm 36^{* * *}$ & $0.61 \pm 0.01^{*}$ & $12 \pm 2^{*}$ \\
\hline Treated/unmodified Ratio & $4.3 \pm 0.4$ & $1.9 \pm 0.2$ & $0.91 \pm 0.03$ & $0.6 \pm 0.2$ \\
\hline
\end{tabular}

Significantly different than unmodified: ${ }^{*}$ paired t test, $p \leq 0.05,{ }^{* * *}$ paired $t$ test, $p \leq 0.001,{ }^{* \star *}$ paired $\mathrm{t}$ test, $\mathrm{p} \leq 0.0001$

The current vs time traces allow a comparison of the time response before and after treatment. There was no delay in the response to a bolus of dopamine compared to unmodified CNTYMEs after 1 min treatment $\mathrm{O}_{2}$ plasma treatment, as seen on the current versus time curve after plasma treatment (Fig. 4.1B), with an average $10-90 \%$ signal rise time of $1.1 \pm 0.2(n=4)$ and $1.2 \pm 0.2(n=4)$ before and after treatment, respectively. Similarly the anti-static gun treated electrode in Figure 4.3E had no delay in the response to a bolus of dopamine, with the $10-90 \%$ signal rise time of $1.2 \pm 0.2(n=4) 1.2 \pm 0.3(n=4)$ before and after treatment, respectively. Therefore, both treatments allow the CNTYME maintains good temporal response with increased sensitivity.

The background currents provide information about electrode surface area enhancements as well as surface functional group changes. The background current increases about 2 fold for both oxygen plasma etching and anti-static gun treatments, which is lower than the increase in the Faradaic current for dopamine. Background voltammetric features at about $-0.1 \mathrm{~V}$ and $1.3 \mathrm{~V}$ 
were obtained at CNTYMEs after $\mathrm{O}_{2}$ plasma etching (Figure 4.1C). In contrast, the background shape did not change much after anti-static gun treatment. In previous work, surface confined species were found to cause feature peaks in the background, such as hydroxyls, carboxylic acids, carbonyls, and lactones. ${ }^{30-32}$ Therefore, the $\mathrm{O}_{2}$ plasma etched are likely to have tremendous amount of functional groups which providing more electroactive site and further improving the sensitivity. In comparison, the charging current shape does not change much after anti-static gun treatment, indicating the detection sensitivity improvement is more likely because of the increasing surface area at anti-static gun treated CNTYMEs. Moreover, since hydrophilic surfaces have larger charging currents than more hydrophobic surface,${ }^{31}$ the larger background increasing after $\mathrm{O}_{2}$ plasma etching $(2.3 \pm 0.4)$ compared to anti-static gun treated CNTYMEs $(1.9 \pm 0.2)$ is likely due to the more hydrophilic surface caused by the abundant surface oxygen-containing functional groups. Because the anti-static gun had the largest increase in dopamine signal and smallest background change, it has a larger signal to background current ratio compared to unmodified and $\mathrm{O}_{2}$ plasma-etched CNTYMEs.

\subsubsection{Physical Characterization of Treated CNTYMEs}

The CNT yarn surfaces with $\mathrm{O}_{2}$ plasma etching or anti-static gun treatment were further physically characterized by SEM, three-dimensional laser scanning confocal microscopy, Raman spectroscopy, and energy-dispersive X-ray spectroscopy (EDS). Figure 4.2 and Figure 4.4 show the SEM images of the same CNTYMEs before and after $\mathrm{O}_{2}$ plasma etching $(1 \mathrm{~min})$ and antistatic gun treatment (2 triggers), respectively. The multi-walled CNTs bundles with a diameter about $30 \mathrm{~nm}$ did not change obviously after $1 \mathrm{~min} \mathrm{O}_{2}$ plasma etching. In comparison, after 2 triggers of the antistatic gun treatment, the CNTs spikes stands up straighter due to the ion flow applied on the surface, providing a larger surface area. Laser scanning laser scanning confocal microscopy was applied to measure the surface roughness precisely. It has advantages of a large measuring range and no requirement of a leveled surface. Table 4.4 summarizes the averaged 
mean roughness depth $\left(R_{z}\right)$, defined as the average distance between the highest peak and lowest valley in each sampling length, at unmodified, $\mathrm{O}_{2}$ plasma etched, and anti-static gun treated CNTYMEs. The surface roughness did not change significantly after $\mathrm{O}_{2}$ plasma etching (unpaired t-test, $\mathrm{p}>0.05, \mathrm{n}=4$ ). In contrast, CNTYMEs with anti-static gun treatment had a significantly larger surface roughness than both unmodified and $\mathrm{O}_{2}$ plasma etched CNTYMEs (one-way ANOVA, Bartlett's test, $\mathrm{p} \leq 0.05, \mathrm{n}=4$ ).

Table 4.4: Surface properties of CNTYMEs with different surface modifications ${ }^{\alpha}$

\begin{tabular}{|c|c|c|c|}
\hline & $\begin{array}{c}\text { Surface Roughness } \\
\mathrm{R}_{\mathrm{z}} / \mathrm{nm}\end{array}$ & Raman D/G Ratio & Oxygen Content \% \\
\hline $\begin{array}{c}\text { Unmodified CNTYME }(\mathrm{n}=4) \\
1540 \pm 160\end{array}$ & $0.72 \pm 0.01$ & $10 \pm 1$ \\
\hline $\begin{array}{c}\mathrm{O}_{2} \text { Plasma Etched CNTYME } \\
(\mathrm{n}=4)\end{array}$ & $1800 \pm 212^{* * *}$ & $1.02 \pm 0.03^{* * *}$ & $19 \pm 1^{* * *}$ \\
\hline $\begin{array}{c}\text { Anti-static gun treated } \\
\text { CNTYME }(\mathrm{n}=4)\end{array}$ & $2600 \pm 400^{*}$ & $0.73 \pm 0.01$ & $10.0 \pm 0.5$ \\
\hline
\end{tabular}

aMean roughness depth (Rz), D/G ratio from Raman spectra, and relative oxygen content at unmodified, $\mathrm{O}_{2}$ plasma etched, and anti-static gun treated CNTYMEs. Significantly different than others: * one-way ANOVA, Bartlett's test, $p \leq 0.05,{ }^{* * *}$ one-way ANOVA, Bartlett's test, $p \leq 0.001$

Raman spectroscopy and energy-dispersive X-ray spectroscopy (EDS) were performed to further characterize the surface defect to graphite carbon $(D / G)$ ratio and oxygen content. The ratio of the $D$ and $G$ band area is widely used to evaluate the relative amount of $\mathrm{sp}^{3}$ and $\mathrm{sp}^{2}$ hybridized carbon at carbon nanomaterials. ${ }^{33,34}$ The D/G ratio at $\mathrm{O}_{2}$ plasma etched CNTYMEs was much larger than both unmodified and anti-static gun treated CNTYMEs (Table 4.4, one-way ANOVA, Bartlett's test, $p \leq 0.001, n=4$ ) due to the carbon-carbon bonds broken by the plasma. The $D / G$ ratios after ion arrangement did not change (unpaired t-test, $p>0.05, n=4$ ), as shown in Figure 4.6, indicating there is likely no chemical change on the surface. The oxygen content measured by EDS significantly increased compared to unmodified and anti-static gun treated 
CNTYMEs (Table 4.4, one-way ANOVA, Bartlett's test, $p \leq 0.001, n=4$ ), indicating the introduction of larger amount of $\mathrm{sp}^{2}$ hybridized carbon after $\mathrm{O}_{2}$ plasma etching were further oxidized to surface functional groups. Meanwhile, the anti-static gun treated CNTYMEs share a similar oxygen content with unmodified CNTYMEs (unpaired t-test, $\mathrm{p}>0.05, \mathrm{n}=4$ ).

A. Raman Spectrum - $\mathrm{O}_{2}$ Plasma Etched CNTYME

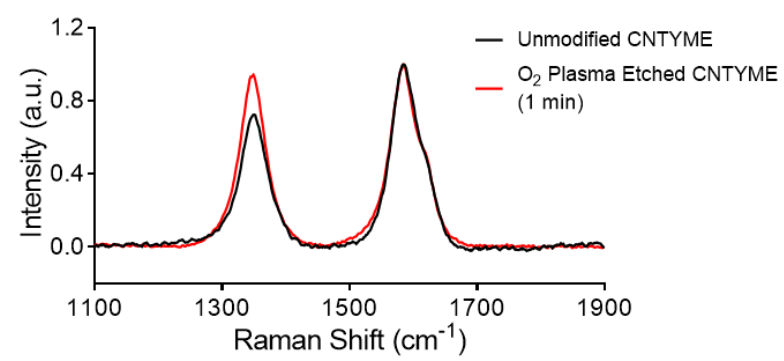

B. Raman Spectrum - Anti-Static Gun Treated CNTYME

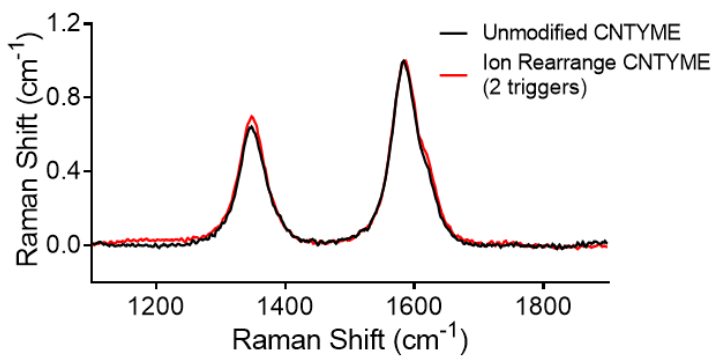

Figure 4.6: Raman spectra obtained at CNTYMEs with different surface modifications. (A) The same electrodes before (black) and after $1 \mathrm{~min}$ (red) $\mathrm{O}_{2}$ plasma etching. (B) A CNTYME before (black) and after 2 triggers (red) anti-static gun treatment. Data was normalized to graphic peak.

Oxygen containing functional groups, such as quinones, hydroxyl, and carboxylic acids are negatively charged at physiological $\mathrm{pH}$ and have electrostatic interactions with positively charged dopamine. ${ }^{7} \mathrm{O}_{2}$ plasma etching introduces more oxygen containing functional groups, providing high dopamine sensitivity and higher surface hydrophilicity, without changing the surface roughness. Oppositely, anti-static gun treatment is a mild surface modification approach for CNTYMEs that does not change the D/G ratio or oxygen content. Instead, the dopamine sensitivity improvement is due only to the increased surface area. To further prove that, carbonfiber microelectrodes not having nanostructure features were treated with different triggers of antistatic gun treatment and tested with $1 \mu \mathrm{M}$ dopamine, as shown in Figure 4.7. Even with up to 8 triggers modification, CFMEs did not show a difference in oxidation current or background current to the same electrode before modification, indicating anti-static gun treatment only modifies 
nanostructures such as CNT bundles in CNT yarn but would not modify smooth electrode surface which is lacking nanostructures, such as carbon fibers.
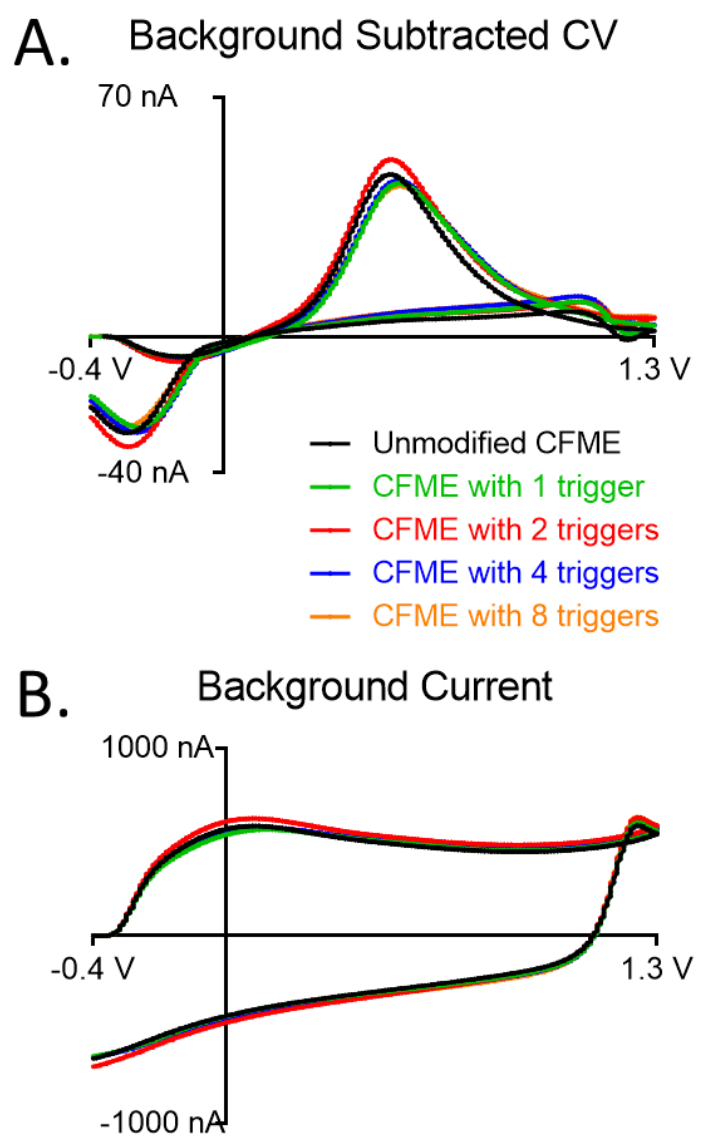

Figure 4.7: Comparison of the response to $1 \mu \mathrm{M}$ dopamine and background current at an unmodified CFME (black line) and the same electrode after 1 (ref line), 2 (green line), 4 (blue line), and 8 (orange line) triggers, respectively. Electrochemical response was measured with scan rate of $400 \mathrm{~V} / \mathrm{s}$ and scan repetition frequency of $10 \mathrm{~Hz}$. (A) Background subtracted cyclic voltammograms to $1 \mu \mathrm{M}$ dopamine, and (B) background currents in PBS buffer. No significant changes are observed.

\subsubsection{Effect of Bio-fouling}

In vivo electrochemical monitoring of neurotransmitters is challenging since the microelectrode surface fouling caused by the adsorption of bio-macromolecules can severely affect the sensitivity and reproducibility of electrochemical sensing in vivo. ${ }^{28,35}$ Carbon 
nanomaterials have been proposed to alleviate surface fouling due to their hydrophilic surface. ${ }^{36,37}$ Here we investigated the bio-fouling effect on CNTYMEs with different surface modifications by comparing dopamine sensitivity before and after placing the microelectrodes in rat brain tissue for two hours without potential cycling, since Singh et al. found there was no difference for 2 hour exposures with or without potential cycling and overnight exposures to brain tissue. ${ }^{28}$

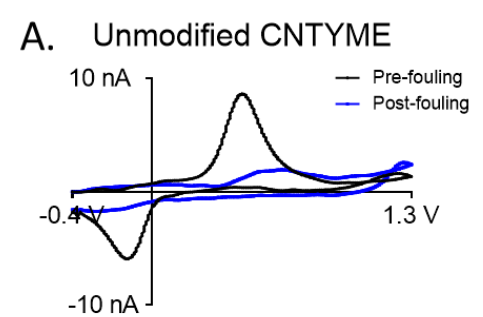

D. Unmodified CNTYME

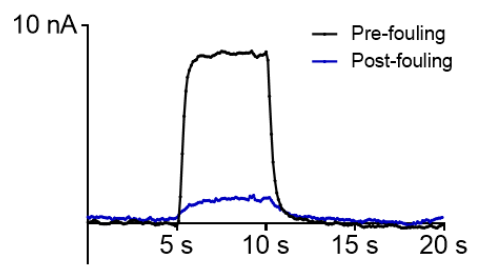

B. $\mathrm{O}_{2}$ Plasma Etched CNTYME

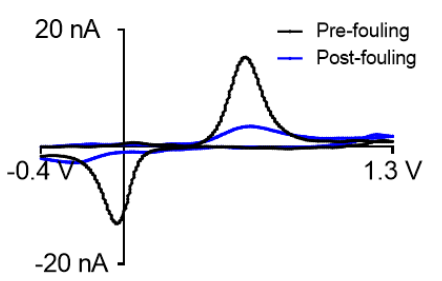

E. $\mathrm{O}_{2}$ Plasma Etched CNTYME

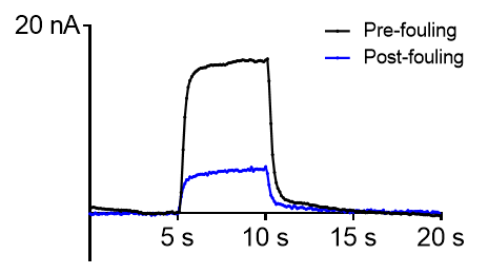

C. Anti-Static Gun Treated CNTYME

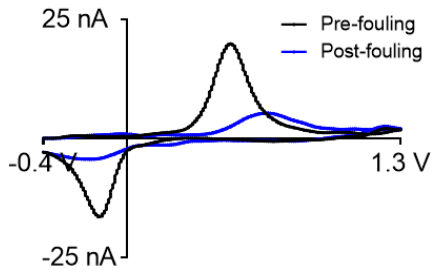

F. Anti-Static Gun Treated CNTYME $25 \mathrm{nA}$

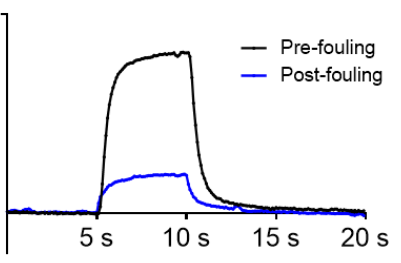

Figure 4.8: Comparison of the response to $1 \mu \mathrm{M}$ dopamine before (black) and after (blue) $2 \mathrm{H}$ intissue fouling, at an unmodified $\operatorname{CNTYME}(A, D), \mathrm{O}_{2}$ plasma etched $\operatorname{CNTYME}(B, E)$, and antistatic gun treated CNTYMEs (C, F). (A-C) Background subtracted cyclic voltammograms to $1 \mu \mathrm{M}$ dopamine, (D-F) measured oxidation current versus time for a flow injection analysis experiment.

Table 4.8 shows the oxidation current, $\Delta \mathrm{E}_{\mathrm{p}}$, and background current before/after fouling at unmodified, $\mathrm{O}_{2}$ plasma etched, and anti-static gun treated CNTYMEs. After 2 hours in tissue, 1 uM dopamine oxidation currents decreased for all electrodes. However, the oxidation currents $\left(I_{p, a}\right)$ to $1 \mu \mathrm{M}$ dopamine at both $\mathrm{O}_{2}$ plasma etched and anti-static gun treated CNTYMEs are significantly larger than the unmodified CNTYMEs (unpaired t-test, $\mathrm{p} \leq 0.0001, \mathrm{n}=6$, for both comparisons), indicating both surface modification methods improve the sensitivity even after tissue exposure. In addition, the $\Delta \mathrm{E}_{\mathrm{p}}$ values at CNTYMEs after both $\mathrm{O}_{2}$ plasma etching and antistatic gun treatment are significantly smaller than unmodified CNTYMEs (unpaired t-test, $p \leq 0.01$ 
$(n=6)$, and $p \leq 0.0001(n=6)$, respectively), indicating the electron transfer is faster at modified CNTYMEs than unmodified electrodes. With about $30 \%$ signal remained after fouling, the $\mathrm{O}_{2}$ plasma etched CNTYMEs show the largest percentage of remained signal, which is significantly better than unmodified CNTYMEs which retain on $18 \%$ of their signal (unpaired t-test, $p \leq 0.05, n$ =6). The percentage of current remaining after fouling at anti-static gun treated CNTYMEs is not significantly different than unmodified CNTYMEs (unpaired t-test, $p>0.05, n=6$ ). Since the current before fouling at anti-static gun treated CNTYMEs is larger than $\mathrm{O}_{2}$ plasma etched and unmodified CNTYMEs (Table 4.5), the current remained after fouling is significantly larger at antistatic gun treated CNTYMEs than that at the $\mathrm{O}_{2}$ plasma etched CNTYMEs (unpaired t-test, $\mathrm{p} \leq$ $0.05, \mathrm{n}=6$ ). The signal remaining after fouling at anti-static gun treated CNTYMEs is the same as the signal at unmodified CNTYMEs without fouling.

Table 4.5: Comparison of dopamine detection at CNTYMEs before/after in tissue fouling ${ }^{\beta}$

\begin{tabular}{|c|c|c|c|c|c|}
\hline & $\begin{array}{c}\text { Initial } \\
\mathrm{I}_{\mathrm{p}, \mathrm{a}} / \mathrm{nA}\end{array}$ & $\begin{array}{c}\mathrm{I}_{\mathrm{p}, \mathrm{a}} \text { after } \\
\text { Fouling/nA }\end{array}$ & $\begin{array}{c}\mathrm{I}_{\mathrm{p}, \mathrm{a}} \\
\text { Ratio(Post/Pre } \\
\text { Fouling)/\% }\end{array}$ & $\begin{array}{c}\Delta \mathrm{E}_{\mathrm{p}} \text { after } \\
\text { Fouling/V }\end{array}$ & $\begin{array}{c}\text { Background } \\
\text { Current } \\
\text { Ratio(Post/Pre } \\
\text { Fouling)/\% }\end{array}$ \\
\hline $\begin{array}{c}\text { Unmodified } \\
\text { CNTYME (n=6) }\end{array}$ & $5.3 \pm 0.5$ & $0.95 \pm 0.05$ & $18 \pm 3$ & $\begin{array}{c}0.923 \pm \\
0.003\end{array}$ & $103 \pm 4$ \\
\hline $\begin{array}{c}\mathrm{O}_{2} \text { Plasma } \\
\text { Etched } \\
\text { CNTYME (n=6) }\end{array}$ & $15 \pm 2$ & $4.5 \pm 0.2^{* * * *}$ & $30 \pm 3^{*}$ & $\begin{array}{c}0.86 \pm \\
0.01^{* *}\end{array}$ & $99 \pm 3$ \\
\hline $\begin{array}{c}\text { Anti-static } \\
\text { gun treated } \\
\text { CNTYME (n=6) }\end{array}$ & $21 \pm 3$ & $5.3 \pm 0.4^{* * * *}$ & $25 \pm 3$ & $\begin{array}{c}0.860 \pm \\
0.006^{* * * *}\end{array}$ & $96 \pm 5$ \\
\hline
\end{tabular}

${ }^{\beta}$ Oxidation current before and after $2 \mathrm{H}$ fouling, the $\mathrm{I}_{\mathrm{p}, \mathrm{a}}$ post/pre fouling ratio, $\Delta \mathrm{E}_{\mathrm{p}}$ value after fouling, and background current post/pre fouling ratio. Significantly different than unmodified: *paired $t$ test, $p \leq 0.05,{ }^{* *}$ paired t test, $p \leq 0.01,{ }^{* * *}$ paired t test, $p \leq 0.0001$.

Compared to hydrophobic surface, hydrophilic surface would reduce the fouling in tissue. ${ }^{36}$ Hydrophobic surfaces promote adsorption of hydrophobic molecules such as aromatic compounds and proteins, and these hydrophobic interactions are sufficiently favorable, that they 
are typically irreversible, especially in an aqueous condition..$^{30}$ In contrast, fouling caused by hydrophilic interactions tends to be more reversible. ${ }^{38}$ Because albumin accounts for more than $50 \%$ of total plasma protein content and is known to adsorb on more hydrophobic surface, ${ }^{39,40}$ increasing the electrode surface hydrophilicity reduces the effect of bio-fouling. The significantly increased amount of oxygen-containing functional groups at CNT yarn surface after $\mathrm{O}_{2}$ plasma etching helps improving the surface hydrophilicity, providing $\mathrm{O}_{2}$ plasma etched CNTYMEs the highest percentage of remained signal after in tissue fouling. Anti-static gun treatment also enhances the anti-fouling property compared to unmodified CNT yarn, but the improvement is less than $\mathrm{O}_{2}$ plasma etching. This can be explained by the absolute and relative amount of surface functional groups: the absolute amount of surface functional groups increases after anti-static gun treatment with the surface area increasing that providing the better dopamine sensitivity and LOD than unmodified CNTYMEs, but the density of functional groups does not change after modification. In comparison, $\mathrm{O}_{2}$ plasma etched CNTYMEs have the highest density of surface oxide groups, which provide the best anti-fouling property among the CNTYMEs with three different surface modifications.

Notably, the background current did not change significantly after fouling in tissue. This result is consistent with previous work that the background current did not significantly change at CFMEs and the electrodes with polymers coating after fouling. ${ }^{28}$ The reason for the unchanged background current after fouling is still elusive, but one possibility might be a synergistic effect of decreased hydrophilicity by protein blocking and increased overall capacitance by increased overall surface area due to protein anchor. ${ }^{41-45}$ In addition, at the unmodified, $\mathrm{O}_{2}$ plasma etched and anti-static gun treated CNTYMEs, the temporal response was not significantly slowed due to fouling, demonstrated by the rapid uptake and release.

This paper introduces $\mathrm{O}_{2}$ plasma etching and anti-static gun treatment as two new, simple but efficient surface modification methods on CNTYMEs. $\mathrm{O}_{2}$ plasma etching was applied on CNT yarn for its electrochemical application and anti-static gun treatment was used as a novel 
nanomaterial surface modification method for the first time. Both treatment significantly improve detection sensitivity to dopamine and the antifouling property, tune surface electrochemistry differently, and enable CNT yarn for sensitive in vivo detections. 


\subsection{Conclusions}

In this work, we introduced and optimized two simple, rapid, reproducible, and efficient surface modification methods for CNT yarn microelectrodes: $\mathrm{O}_{2}$ plasma etching and anti-static gun treatment. $\mathrm{O}_{2}$ plasma etching is first applied on CNT yarn for its electrochemical sensing application. Anti-static gun treatment is a novel, low-cost, simple, all-levels-lab feasible surface modification method, which is for the first time used for nanomaterials. Both of the surface modification methods significantly improve the dopamine detection signals by three to four fold. In addition, since the $\mathrm{O}_{2}$ plasma etching and anti-static gun treatment specifically increase the amount of oxygen content and surface roughness, respectively, $\mathrm{O}_{2}$ plasma etched and anti-static gun treated CNTYMEs provide promising platforms for the study on how each surface property influence the electrochemistry characteristics. The effect of bio-fouling was evaluated at CNTYMEs for the first time, and oxide-groups-abundant $\mathrm{O}_{2}$ plasma etched CNTYMEs are proven to have better anti-fouling property. This work not only provides two surface modification methods which could significantly enhance CNTYMEs sensitivity and anti-fouling property, but also provide a approach for future study on the interaction between CNT surface and neurotransmitters. 


\subsection{References}

(1) Vilatela, J. J.; Marcilla, R. Tough Electrodes: Carbon Nanotube Fibers as the Ultimate Current Collectors/Active Material for Energy Management Devices. Chem. Mater. 2015, 27 (20), 69016917.

(2) Lu, W.; Zu, M.; Byun, J. H.; Kim, B. S.; Chou, T. W. State of the Art of Carbon Nanotube Fibers: Opportunities and Challenges. Adv. Mater. 2012, 24 (14), 1805-1833.

(3) Schmidt, A. C.; Wang, X.; Zhu, Y.; Sombers, L. a. Carbon Nanotube Yarn Electrodes for Enhanced Detection of Neurotransmitter Dynamics in Live Brain Tissue. ACS Nano 2013, 7864-7873.

(4) Harreither, W.; Trouillon, R.; Poulin, P.; Neri, W.; Ewing, A. G.; Safina, G. Carbon Nanotube Fiber Microelectrodes Show a Higher Resistance to Dopamine Fouling. Anal. Chem. 2013, 85 (15), 74477453.

(5) Yang, C.; Jacobs, C. B.; Nguyen, M. D.; Ganesana, M.; Zestos, A. G.; Ivanov, I. N.; Puretzky, A. A.; Rouleau, C. M.; Geohegan, D. B.; Venton, B. J. Carbon Nanotubes Grown on Metal Microelectrodes for the Detection of Dopamine. Anal. Chem. 2016, 88 (1), 645-652.

(6) Yang, C.; Denno, M. E.; Pyakurel, P.; Venton, B. J. Recent Trends in Carbon Nanomaterial-Based Electrochemical Sensors for Biomolecules: A Review. Anal. Chim. Acta 2015, 887, 17-37.

(7) Roberts, J. G.; Moody, B. P.; McCarty, G. S.; Sombers, L. a. Specific Oxygen-Containing Functional Groups on the Carbon Surface Underlie an Enhanced Sensitivity to Dopamine at Electrochemically Pretreated Carbon Fiber Microelectrodes. Langmuir 2010, 26 (11), 9116-9122.

(8) Jacobs, C. B.; Ivanov, I. N.; Nguyen, M. D.; Zestos, A. G.; Venton, B. J. High Temporal Resolution Measurements of Dopamine with Carbon Nanotube Yarn Microelectrodes. Anal. Chem. 2014, 86 (12), 5721-5727.

(9) Zestos, A. G.; Jacobs, C. B.; Trikantzopoulos, E.; Ross, A. E.; Venton, B. J. Polyethylenimine Carbon Nanotube Fiber Electrodes for Enhanced Detection of Neurotransmitters. Anal. Chem. 2014, 86, 8568-8575.

(10) Jacobs, C. B.; Vickrey, T. L.; Venton, B. J. Functional Groups Modulate the Sensitivity and Electron Transfer Kinetics of Neurochemicals at Carbon Nanotube Modified Microelectrodes. Analyst 2011, 136 (17), 3557.

(11) Takmakov, P.; Zachek, M. K.; Keithley, R. B.; Walsh, P. L.; Donley, C.; McCarty, G. S.; Wightman, R. M. Carbon Microelectrodes with a Renewable Surface. Anal. Chem. 2010, 82 (5), 2020-2028.

(12) Bartosova, Z.; Riman, D.; Halouzka, V.; Vostalova, J.; Simanek, V.; Hrbac, J.; Jirovsky, D. A Comparison of Electrochemically Pre-Treated and Spark-Platinized Carbon Fiber Microelectrode. Measurement of 8-Oxo-7,8-Dihydro-2'-Deoxyguanosine in Human Urine and Plasma. Anal. Chim. Acta 2016, 2-9.

(13) Ross, A. E.; Venton, B. J. Nafion-CNT Coated Carbon-Fiber Microelectrodes for Enhanced Detection of Adenosine. Analyst 2012, 137 (13), 3045-3051.

(14) Peairs, M. J.; Ross, A. E.; Venton, B. J. Comparison of Nafion- and Overoxidized Polypyrrole-Carbon Nanotube Electrodes for Neurotransmitter Detection. Anal. Methods 2011, 3 (10), 2379.

(15) Xu, G.; Li, B.; Cui, X. T.; Ling, L.; Luo, X. Electrodeposited Conducting Polymer PEDOT Doped with 
Pure Carbon Nanotubes for the Detection of Dopamine in the Presence of Ascorbic Acid. Sensors Actuators B Chem. 2013, 188 (2013), 405-410.

(16) Gu, L.; Jiang, X.; Liang, Y.; Zhou, T.; Shi, G. Double Recognition of Dopamine Based on a Boronic Acid Functionalized Poly(aniline-Co-Anthranilic Acid)-Molecularly Imprinted Polymer Composite. Analyst 2013, 138 (18), 5461-5469.

(17) Liu, B.; Lian, H. T.; Yin, J. F.; Sun, X. Y. Dopamine Molecularly Imprinted Electrochemical Sensor Based on Graphene-chitosan Composite. Electrochim. Acta 2012, 75 (2012), 108-114.

(18) Yang, C.; Trikantzopoulos, E.; Nguyen, M. D.; Jacobs, C. B.; Wang, Y.; Mahjouri-Samani, M.; Ivanov, I. N.; Venton, B. J. Laser Treated Carbon Nanotube Yarn Microelectrodes for Rapid and Sensitive Detection of Dopamine in Vivo. ACS Sensors 2016, 1 (5), 508-515.

(19) Strein, T. G.; Ewing, A. G. In Situ Laser Activation of Carbon Fiber Microdisk Electrodes. Anal. Chem. 1991, 63, 194-198.

(20) Strein, T. G.; Ewing, a G. Laser Activation of Microdisk Electrodes Examined by Fast-Scan Rate Voltammetry and Digital-Simulation. Anal. Chem. 1994, 66 (28), 3864-3872.

(21) E, S. P.; Miller, T. S.; Macpherson, J. V.; Unwin, P. R. Controlled Functionalisation of Single-Walled Carbon Nanotube Network Electrodes for the Enhanced Voltammetric Detection of Dopamine. Phys. Chem. Chem. Phys. 2015, 17 (39), 26394-26402.

(22) Claussen, J. C.; Kumar, A.; Jaroch, D. B.; Khawaja, M. H.; Hibbard, A. B.; Porterfield, D. M.; Fisher, T. S. Nanostructuring Platinum Nanoparticles on Multilayered Graphene Petal Nanosheets for Electrochemical Biosensing. Adv. Funct. Mater. 2012, 22 (16), 3399-3405.

(23) Sharma, P.; Bernard, L. S.; Bazigos, A.; Magrez, A.; lonescu, A. M. Room-Temperature Negative Di Ff Erential Resistance in Graphene Field E Ff Ect Transistors : Experiments and Theory. 2014, 0-5.

(24) Zhao, J.; Zhang, W.; Sherrell, P.; Razal, J. M.; Huang, X.-F.; Minett, A. I.; Chen, J. Carbon Nanotube Nanoweb-Bioelectrode for Highly Selective Dopamine Sensing. ACS Appl. Mater. Interfaces 2012, $4(1), 44-48$.

(25) Xue, Q.; Kato, D.; Kamata, T.; Umemura, S.; Hirono, S.; Niwa, O. Electron Cyclotron ResonanceSputtered Nanocarbon Film Electrode Compared with Diamond-like Carbon and Glassy Carbon Electrodes as Regards Electrochemical Properties and Biomolecule Adsorption. Jpn. J. Appl. Phys. 2012, 51 (9).

(26) Valentini, F.; Romanazzo, D.; Carbone, M.; Palleschi, G. Modified Screen-Printed Electrodes Based on Oxidized Graphene Nanoribbons for the Selective Electrochemical Detection of Several Molecules. Electroanalysis 2012, 24 (4), 872-881.

(27) Melechko, a. V.; Merkulov, V. I.; McKnight, T. E.; Guillorn, M. a.; Klein, K. L.; Lowndes, D. H.; Simpson, M. L. Vertically Aligned Carbon Nanofibers and Related Structures: Controlled Synthesis and Directed Assembly. J. Appl. Phys. 2005, 97 (4), 41301.

(28) Singh, Y. S.; Sawarynski, L. E.; Dabiri, P. D.; Choi, W. R.; Andrews, A. M. Head-to-Head Comparisons of Carbon Fiber Microelectrode Coatings for Sensitive and Selective Neurotransmitter Detection by Voltammetry. Anal. Chem. 2011, 83 (17), 6658-6666.

(29) Nguyen, M. D.; Lee, S. T.; Ross, A. E.; Ryals, M.; Choudhry, V. I.; Venton, B. J. Characterization of 
Spontaneous, Transient Adenosine Release in the Caudate-Putamen and Prefrontal Cortex. PLoS One 2014, 9 (1), e87165.

(30) McCreery, R. L. Advanced Carbon Electrode Materials for Molecular Electrochemistry. Chem. Rev. 2008, 108 (7), 2646-2687.

(31) Runnels, P. L.; Joseph, J. D.; Logman, M. J.; Wightman, R. M. Effect of pH and Surface Functionalities on the Cyclic Voltammetric Responses of Carbon-Fiber Microelectrodes. Anal. Chem. 1999, 71 (14), 2782-2789.

(32) Evans, J. F.; Kuwana, T.; Henne, M. T. Electrocatalysis of Solution Species Using Modified Electrodes. J. Electroanal. Chem. 1977, 80, 409-416.

(33) Filik, J.; May, P. W.; Pearce, S. R. J.; Wild, R. K.; Hallam, K. R. XPS and Laser Raman Analysis of Hydrogenated Amorphous Carbon Films. Diam. Relat. Mater. 2003, 12 (3-7), 974-978.

(34) Ivanov, I.; Puretzky, A.; Eres, G.; Wang, H.; Pan, Z.; Cui, H.; Jin, R.; Howe, J.; Geohegan, D. B. Fast and Highly Anisotropic Thermal Transport through Vertically Aligned Carbon Nanotube Arrays. Appl. Phys. Lett. 2006, 89 (22), 223110.

(35) Chen, C.-H.; Luo, S.-C. Tuning Surface Charge and Morphology for the Efficient Detection of Dopamine under the Interferences of Uric Acid, Ascorbic Acid, and Protein Adsorption. ACS Appl. Mater. Interfaces 2015, 7 (39), 21931-21938.

(36) Hanssen, B. L.; Siraj, S.; Wong, D. K. Y. Recent Strategies to Minimise Fouling in Electrochemical Detection Systems. Rev. Anal. Chem. 2016, 35 (1), 1-28.

(37) Harreither, W.; Trouillon, R.; Poulin, P.; Neri, W.; Ewing, A. G.; Safina, G. Carbon Nanotube Fiber Microelectrodes Show a Higher Resistance to Dopamine Fouling. Anal. Chem. 2013, 85 (15), 74477453.

(38) Tang, L.; Thevenot, P.; Hu, W. Surface Chemistry Influences Implant Biocompatibility. Curr. Top. Med. Chem. 2008, 8 (4), 270-280.

(39) Harreither, W.; Trouillon, R.; Poulin, P.; Neri, W.; Ewing, A. G.; Safina, G. Cysteine Residues Reduce the Severity of Dopamine Electrochemical Fouling. Electrochim. Acta 2016, 210, 622-629.

(40) Haskard, C. A.; Li-Chan, E. C. Y. Hydrophobicity of Bovine Serum Albumin and Ovalbumin Determined Using Uncharged (PRODAN) and Anionic (ANS-) Fluorescent Probes. J. Agric. Food Chem. 1998, 46 (97), 2671-2677.

(41) Sun, Y. P.; Fu, K. F.; Lin, Y.; Huang, W. J. Functionalized Carbon Nanotubes: Properties and Applications. Acc. Chem. Res. 2002, 35 (12), 1096-1104.

(42) Prato, M.; Marchesan, S. Under the Lens: Carbon Nanotube and Protein Interaction at the Nanoscale. Chem. Commun. 2015, 51 (21), 4347-4359.

(43) Barbosa, A. F.; Barbosa, V. M. P.; Bettini, J.; Luccas, P. O.; Figueiredo, E. C. Restricted Access Carbon Nanotubes for Direct Extraction of Cadmium from Human Serum Samples Followed by Atomic Absorption Spectrometry Analysis. Talanta 2015, 131, 213-220.

(44) Baldacchini, C.; Herrero Chamorro, M. A.; Prato, M.; Cannistraro, S. Highly Conductive Redox Protein-Carbon Nanotube Complex for Biosensing Applications. Adv. Funct. Mater. 2011, 21 (1), 153-157. 
(45) Xu, H.; Dai, H.; Chen, G. Direct Electrochemistry and Electrocatalysis of Hemoglobin Protein Entrapped in Graphene and Chitosan Composite Film. Talanta 2010, 81 (1-2), 334-338. 
Chapter 5: Evaluation of Carbon Nanotube Fiber Microelectrodes for Neurotransmitter Detection: Correlation of Electrochemical Performance and Surface Properties 


\section{Abstract}

Fibers made of CNTs are attractive microelectrode sensors because they can be directly fabricated into microelectrodes. There are different protocols for making CNT fibers, but how those protocols result in different surface and electrochemical properties has not been identified. In this study, we correlated the surface and electrochemical properties for neurochemical detection at 3 types of materials: CNT fibers produced by wet spinning with (1) polyethylenimine (PEl/CNT) or (2) chlorosulfonic acid (CA/CNT), and (3) CNT yarns made by solid-based CNT drawing. CNT yarns had well-aligned, high purity CNTs, abundant oxygen functional groups, and moderate surface roughness which led to the highest dopamine current density $\left(290 \pm 65 \mathrm{pA} / \mathrm{cm}^{2}\right)$ and fastest electron transfer kinetics. The crevices of the CNT yarn and PEI/CNT fiber microelectrodes allow dopamine to get momentarily trapped during fast-scan cyclic voltammetry detection, leading to thin-layer cell conditions and a response that was independent of applied waveform frequency. The larger crevices on the PEI/CNT fibers led to a slower time response, showing too much roughness is detrimental to fast detection. CA/CNT fibers have a smoother surface, leading to lower currents, but their negative surface charge results in high selectivity for dopamine over uric acid or ascorbic acid. These were the first studies of CA/CNT fibers for biomolecule detection. Overall, small crevices, high conductivity, and abundant oxygen groups lead to high sensitivity for amine neurotransmitters, such as dopamine and serotonin. These results show that surface properties of CNT fibers can be used to predict their electrochemical performance. 


\subsection{Introduction}

Carbon nanotubes (CNTs) have been used as an electrode material for more than a decade. CNTs are attractive for making microelectrodes because of their large surface area, abundant electroactive sites for biomolecules, fast electron transfer kinetics, and minimal electrode fouling. ${ }^{1-4}$ Moreover, the small dimensions of CNTs limit tissue damage for in vivo implantation while maintaining high detection sensitivity due to the high surface-to-volume ratio. 5,6 The commonly used methods to modify CNTs on carbon fiber or metal microelectrodes include dip coating ${ }^{7}$, self-assembled $\mathrm{CNTs}^{4}$, and directly growing CNTs on substrates. ${ }^{8,9}$ Although many of these methods lead to outstanding electrochemical performance, future applications are limited by the cumbersome fabrication that often requires specialized equipment and the relatively low reproducibility. Recently, development of high-performance macro-structures of CNTs, such as CNTs fibers, have led to research on their applications in bio-sensing, since fibers can be handled much more conveniently than individual CNTs. ${ }^{10,11}$ In particular, CNT fibers can be directly fabricated into electrodes, in a manner similar to carbon fiber microelectrodes (CFMEs), dramatically simplifying the fabrication process and improving the reproducibility. ${ }^{5}$

Two distinct protocols have been developed for manufacturing CNT fibers: wet-spinning a suspension of CNTs into a bath or direct spinning a fiber from a CNT forest. In solution based wet spinning, CNTs are either dissolved or dispersed in a fluid, extruded out of a spinneret, and coagulated into a solid fiber by extracting the dispersant. ${ }^{12,13}$ Neat CNT fibers can be spun into a polymer bath, such as poly(vinyl alcohol) $(\mathrm{PVA})^{14}$ or poly(ethylene)imine $(\mathrm{PEI})^{15}$, in which the polymer molecules act as coagulators for CNTs to enhance the intertube interactions. Another variation of the original wet spinning method uses acid as a solvent, simplifying the process by avoiding surfactants in the CNTs dispersion. ${ }^{14,16}$ CNT fibers fabricated by wet spinning with chlorosulfonic acid, which is the only known CNT solvent ${ }^{17,18}$, have the highest conductivity among CNTs fibers. ${ }^{13}$ The alternative fiber production route employs a solid state process where CNTs 
are either directly spun as a fiber from the synthesis reaction zone ${ }^{19}$ or from a CNT forest grown on a solid substrate ${ }^{20}$. CNT yarns, generated by drawing one end of the vertically aligned CNT arrays, are a favorable electrode material because of their well-aligned CNTs and improved conductivity. ${ }^{21,22}$ While several studies have tested the electrochemical properties CNT yarn or PEI/CNT fiber microelectrodes ${ }^{5,23,24}$, there are no reports of CA/CNT fibers for biological applications. In addition, no studies have compared different CNT fibers or correlated their surface and electrochemical properties.

This work is the first to compare different CNT fiber based microelectrodes (PEI/CNT fiber, CA/CNT fiber, and CNTs yarn microelectrodes). CNT yarn microelectrodes provide the highest sensitivity to dopamine and fastest electron transfer kinetics because of the well-aligned, high purity CNTs, abundant oxygen containing functional groups at the surface, and small crevices to trap dopamine for short times. CA/CNT fibers, as a novel electrode material, provide high selectivity to dopamine over uric acid and ascorbic acid because of their negatively charged surface. The frequency independent property of CNT yarn and PEI/CNT fiber microelectrodes is due to reversible dopamine redox reaction when the dopamine gets trapped in the crevices, leading to thin-layer cell conditions. However, the larger crevices on the PEI/CNT fibers lead to a slower time response, showing too much roughness can be detrimental to fast temporal response. This study facilitates a better understanding of how surface properties lead to electrochemical properties, which will enable future predictions of electrochemical properties based on the surface characteristics of carbon nanomaterial-based microelectrodes. 


\subsection{Experimental Section}

\subsubsection{Chemicals and Solutions}

Dopamine hydrochloride, uric acid, ascorbic acid, and 5-hydroxytryptamine were purchased from Sigma-Aldrich (St. Louis, MO). A $10 \mathrm{mM}$ stock solution was prepared in $\mathrm{HClO}_{4}$, and were diluted daily to the desired concentration in phosphate buffered saline $(131.3 \mathrm{mM} \mathrm{NaCl}$, $3.00 \mathrm{mM} \mathrm{KCl}, 10 \mathrm{mM} \mathrm{NaH}_{2} \mathrm{PO}_{4}, 1.2 \mathrm{mM} \mathrm{MgCl}_{2}, 2.0 \mathrm{mM} \mathrm{Na}_{2} \mathrm{SO}_{4}$, and $1.2 \mathrm{mM} \mathrm{CaCl}_{2}$ with the pH adjusted to 7.4$)$.

\subsubsection{PEI/CNT Fiber and CA/CNT Fiber Preparation}

PEI/CNT fibers were formed as previously described. ${ }^{15}$ HiPCo CNTs (1.2 w\%) were suspended in water with sodium dodecylbenzenesulfonate $(1.2 \mathrm{w} \%)$ and were pumped at a rate of $0.5 \mathrm{~mL} / \mathrm{min}$ into a rotating solution of $40 \% \mathrm{PEI}$ (branched, MW $=50000-100000, \mathrm{MP}$ Biomedicals, LLC, Santa Ana, CA) in methanol. The CNT ribbons were subsequently purified in methanol. CNT fibers were dried in air and then $180^{\circ} \mathrm{C}$ for $1 \mathrm{~h}$.

CA/CNT fibers were formed as previously described. ${ }^{13}$ HiPCo CNTs were dissolved in chlorosulfonic acid at a concentration of $1.2 \mathrm{w} \%$ (same as for PEI/CNT) to form a spinnable liquid crystal dope (with CNT concentration more than $1.2 \mathrm{w} \%$, the CA/CNT fiber microelectrodes

produced were electrochemically noisy and unstable). The dope was extruded through a spinneret at a rate of $0.5 \mathrm{~mL} / \mathrm{min}$ into acetone to remove the acid. The fibers were dried in room conditions for five hours under constant stretching and tensioning from both ends.

\subsubsection{CNT Fiber and Yarn Microelectrode Fabrication}

A $0.68 \mathrm{~mm}$ ID $\times 1.2 \mathrm{~mm}$ OD (A-M Systems, Carlsborg, WA) glass capillary was pulled into a glass pipet puller and cut to have an opening diameter of $\sim 50 \mu \mathrm{m}$. A piece of either PEI/CNT fiber, CA/CNT fiber, or CNT yarn (15-25 $\mu \mathrm{m}$ in diameter, 1-2 cm long, General Nano, LLC, 
Cincinnati, $\mathrm{OH}$ ) was inserted into the glass pipette. The electrodes were epoxied with Epon Resin 828 (Miller-Stephenson, Danbury, CT) mixed with 14 weight\% 1,3-phenylenediamine hardener (Sigma-Aldrich, St. Louis, MO) heated to $85^{\circ} \mathrm{C}$. The epoxied electrodes were cured overnight at room temperature and then heated at $100^{\circ} \mathrm{C}$ for $2 \mathrm{~h}$ and at $150^{\circ} \mathrm{C}$ overnight. Microelectrodes were polished at a $90^{\circ}$ angle on a fine diamond abrasive plate (Sutter Instruments model BV-10, Novato, CA). Disk CFMEs were fabricated using 7- $\mu$ m-diameter T-650 carbon fibers (Cytec Technologies, Woodland Park, NJ). ${ }^{23}$

\subsubsection{Surface Characterization}

Scanning electron microscope (SEM) images were taken on Merlin field emission SEM (Zeiss, Thornwood, NY) with a secondary electron detector using an accelerating voltage of $2 \mathrm{kV}$ and a working distance of $5.0 \mathrm{~mm}$. Energy dispersive X-ray spectroscopy (EDS) data was acquired using a JEOL JSM-6700F cold field-emission microscope (Tokyo, Japan). The EDS spectra were acquired for 60 seconds at the tip of microelectrodes with an acceleration voltage of $15 \mathrm{kV}$ at a working distance of $5 \mathrm{~mm}$.

\subsubsection{Electrochemical Measurements}

FSCV was performed with a ChemClamp potentiometer (Dagan, Minneapolis, MN, with 1 MOhm Headstage). The waveform was generated and the data was collected using a High Definition Cyclic Voltammetry (HDCV) breakout box, HDCV analysis software program (UNC Chemistry Department, Electronics Design Facility) and PCle-6363 computer interface cards (National Instruments, Austin, TX). Electrodes were backfilled with $1 \mathrm{M} \mathrm{KCl}$ and a silver wire was inserted to connect the electrode to the potentiostat headstage. The typical triangular waveform swept the applied potential from $-0.4 \mathrm{~V}$ to $1.3 \mathrm{~V}$ at $400 \mathrm{~V} / \mathrm{s}$ versus an $\mathrm{Ag} / \mathrm{AgCl}$ reference electrode, at a scan repetition frequency of $10 \mathrm{~Hz}$. The repetition rate was varied for some experiments. 
Electrodes were tested using a flow-injection system. Analyte was injected for 5 seconds (varied for some experiment) and current versus time traces were obtained by integrating the current in a $100 \mathrm{mV}$ window centered at the oxidation peak for each cyclic voltammogram (CV). Background-subtracted CVs were calculated by subtracting the average of 10 background scans, taken before the compound was injected, from the average of five CVs recorded after the analyte bolus was injected. A four-point probe method was used for conductivity determinations.

\subsubsection{Langmuir isotherm modeling}

We used a Langmuir adsorption isotherm (Eq. 1) to model the adsorption and desorption process kinetics of dopamine, as reported previously. ${ }^{9}$ Compared to the dynamic process simulation reported previously ${ }^{25}$, Langmuir isotherm modeling takes the limited amount of adsorption sites on microelectrodes surface into account.

$$
\frac{\Gamma_{D A}}{\Gamma_{S}}=\frac{\beta_{D A} a_{D A}^{b}}{1+\beta_{D A} a_{D A}^{b}}
$$

$\Gamma_{\mathrm{DA}}$ is the amount of dopamine adsorbed on the electrode, $\Gamma_{\mathrm{s}}$ is the saturated amount of dopamine that can adsorb on the electrode, $\beta_{\mathrm{DA}}$ is the thermodynamic equilibrium constant (unitless) for dopamine, and $a_{D A}^{b}$ is the activity of dopamine in bulk solution at equilibrium. The percent surface coverage, $\Gamma_{\mathrm{DA}} / \Gamma_{\mathrm{s}}$, can be expressed by the ratio of the oxidation current of dopamine to the theoretical saturated oxidation current which is the theoretical plateau of the fitting curve. Currents were read till the adsorption/desorption reached equilibrium conditions. The activity is related to its molar concentration $\left(C_{\mathrm{DA}}\right)$ by the following equation: ${ }^{26}$

$$
a_{D A}=\left(\gamma_{D A} \cdot C_{D A}\right) \cdot\left(1 L \cdot \mathrm{mol}^{-1}\right) \quad(\text { Eq. 5.2) }
$$

$\gamma_{D A}$ is the activity coefficient of dopamine in bulk solution at the adsorption equilibrium. For a charged adsorbate solution at high concentration, the effect of the activity coefficients must be taken into account because charged adsorbates are governed by ionic interactions. ${ }^{27}$ According to the Debye-Huckel law: 


$$
\log \gamma_{D A}=-A z^{2} I^{\frac{1}{2}} \text { (Eq. 5.3) }
$$

$\gamma_{D A}$ is a function of the ionic strength $(I)$ of the solute and the charge carried by each solute (z), $A$ is a constant that depends on temperature and is about 0.51 for water at $25^{\circ} \mathrm{C} .{ }^{28}$ Thus, $\gamma_{D A}$ for dopamine in PBS buffer is 0.63 at room temperature. ${ }^{29}$

\subsubsection{Statistics}

All values are given as mean \pm standard error of the mean (error bar) for $\mathrm{n}$ number of electrodes. Paired or unpaired t tests were performed to compare properties between two groups. A one-way ANOVA with Bonferonni post-tests was used to compare effects among multiple groups. All statistics were performed in GraphPad Prism6 (GraphPad Software,Inc., La Jolla, CA). Scanning electron microscopy images were processed using ImageJ (Rasband, W.S., National Institutes of Health, Bethesda, MD). 


\subsection{Results and Discussions}

\subsubsection{Surface Comparison of CNT Fiber Microelectrodes}

CNT fibers were fabricated as microelectrodes and polished vertically at the tips, which allows physical characterizations of the electroactive surface, instead of the entire bulk fiber. $\mathrm{CA} / \mathrm{CNT}$ and PEI/CNT fibers were constructed by wet spinning procedures, as reported previously. ${ }^{13,15}$ The diameter of CA/CNT and PEI/CNT fibers is dependent on the flow rate of the syringe pump and the rotation speed of the stage. The diameters of both fibers were $\sim 20 \mu \mathrm{m}$, as shown in Figure 5.1A and B, which is similar to the diameter of CNT yarn (Fig. 5.1C). Chlorosulfonic acid is the first true solvent for CNTs. A solution, rather than a suspension, of CNTs could lead to improved CNTs alignment and packing of the CA/CNT fibers. ${ }^{13}$ The CA/CNT fiber retained its circular shape and coagulated in a uniform manner (Fig. 5.1A), with a substructure of well packed CNT super-ropes, approximately $200-500 \mathrm{~nm}$ in diameter, connected to one another (Fig. 5.1D). PEI/CNT fibers are ribbon-like gel fibers that partially collapse when drying to form tubular structures. ${ }^{15}$ The surface of the PEI/CNT fiber is primarily composed of SWCNTs with distinct regions of PEI that are not fully removed during rinsing. The outer fiber surface is corrugated, with the axis of corrugation lying approximately parallel to the fiber axis (Fig. 5.1B). These corrugations with diameters about $0.5-1 \mu \mathrm{m}$ are typically observed along the outer surface of fibers and could be also seen on the polished microelectrode surface (Fig. 5.1E). The CNT yarn (Fig. 5.1C) was approximately $20 \mu \mathrm{m}$ in diameter and was spun from a forest of $40-50 \mathrm{~nm}$ diameter individual multiwalled CNTs. ${ }^{24}$ The CNTs bundles, which are the ends of CNTs, show the relatively rough surface on CNT yarn microelectrode tip (Fig. 5.1F). The surfaces of the PEI/CNT fiber and CNT yarn are much rougher than the surface of CA/CNT fiber. 

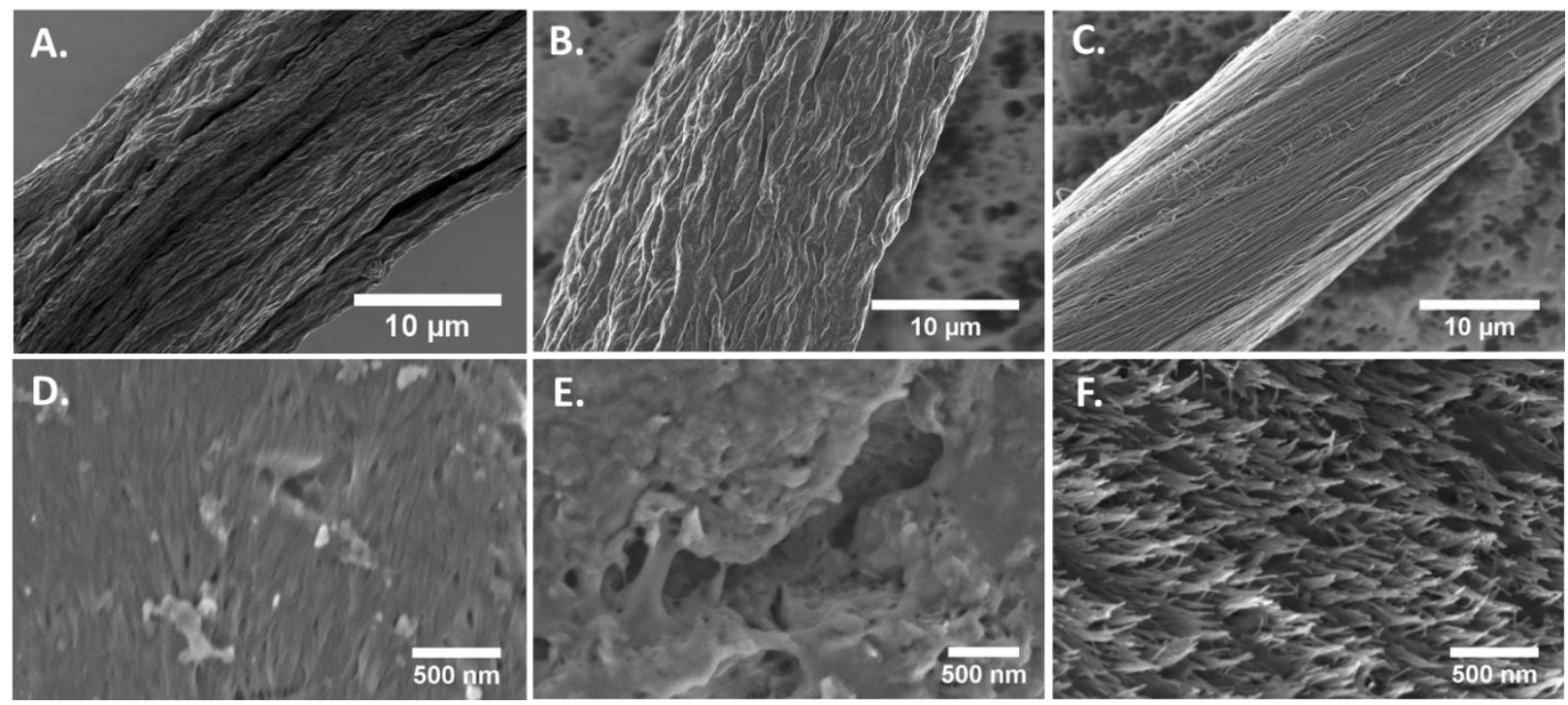

Figure 5.1: Surfaces of microelectrodes. SEM images of the (A) CA/CNT fiber, (B) PEI/CNT fiber, and $(\mathrm{C})$ CNT yarn sidewall, with the diameters about $20 \mu \mathrm{m}$. Scale bar: $10 \mu \mathrm{m}$. Top-view SEM images at the tips of polished (D) CA/CNT fiber, (E) PEI/CNT fiber, and (F) CNT yarn microelectrodes. Scale bar: $500 \mathrm{~nm}$.

Table 5.1: Elemental Contents at the tips of Microelectrodes Measured by EDS

\begin{tabular}{|c|c|c|c|c|c|c|}
\hline & $\mathrm{C} / \%$ & $\mathrm{O} / \%$ & $\mathrm{Fe} / \%$ & $\mathrm{~S} / \%$ & $\mathrm{Cl} / \%$ & $\mathrm{~N} / \%$ \\
\hline CA/CNT Fiber & $79 \pm 2$ & $11 \pm 1$ & $2.1 \pm 0.1$ & $3 \pm 1$ & $2 \pm 1$ & $\mathrm{~N} / \mathrm{A}$ \\
\hline PEI/CNT Fiber & $66.8 \pm 0.2$ & $12.6 \pm 0.5$ & $3.2 \pm 0.2$ & $0.43 \pm 0.05$ & $\mathrm{~N} / \mathrm{D}$ & $16.4 \pm 0.2$ \\
\hline CNT Yarn & $81 \pm 1$ & $19 \pm 1$ & $\mathrm{~N} / \mathrm{D}$ & $\mathrm{N} / \mathrm{D}$ & $\mathrm{N} / \mathrm{D}$ & $\mathrm{N} / \mathrm{D}$ \\
\hline
\end{tabular}

$\mathrm{N} / \mathrm{D}:$ not detected

The elemental content was measured at the tip of polished microelectrodes by energydispersive X-ray spectroscopy (EDS), as shown in Table 5.1. The CA/CNT fiber has small amount of sulfur and chlorine, which likely come from residual chlorosulfonic acid. The large amount of nitrogen on the PEI/CNT fiber comes from PEI which was not fully removed during rinsing and the small amount of sulfur from the sulfur-containing anionic surfactants in the suspension solution. PEI/CNT fibers have more residue than CA/CNT fibers. Both the CA/CNT and PEI/CNT fibers have iron impurities from HiPco SWCNTs. In contrast, the CNT yarns are relatively pure, with no 
polymer, surfactant residue, or iron impurities. Moreover, the oxygen content at CNT yarn surface is significantly larger than at CA/CNT and PEI/CNT fibers (unpaired t-test, $p \leq 0.01$ for both comparisons).

The conductivity was measured at each fiber using four-point probe method. The CNT yarn has highest conductivity, $1025 \pm 25 \mathrm{~S} \cdot \mathrm{cm}^{-1}(\mathrm{n}=3)$, which is in agreement with the literature value range $\left(500-1250 \mathrm{~S} \cdot \mathrm{cm}^{-1}\right) \cdot{ }^{30}$ The conductivity of our CA/CNT fibers, $618 \pm 23 \mathrm{~S} \cdot \mathrm{cm}^{-1}$, was not as good as CA/CNT fiber reported previously $\left(2900 \pm 300 \mathrm{~S} \cdot \mathrm{cm}^{-1}\right) \cdot{ }^{13}$ The lower conductivity might be caused by different SWCNTs having more iron impurity, as indicated by EDS measurements. PEI/CNT fibers have the lowest conductivity, $16.4 \pm 0.4 \mathrm{~S} \cdot \mathrm{cm}^{-1}(\mathrm{n}=3)$, similar to previous work $\left(10-30 \mathrm{~S} \cdot \mathrm{cm}^{-1}\right) \cdot{ }^{15}$ Although PEl is an ionically conductive polymer, its conductivity is not as high as pure CNTs so the residual PEI would decrease the overall conductivity. The high-to-low trend of conductivity: CNT yarn, CA/CNT fiber, and PEI/CNT fiber, is consistent with the high-to-low trends of alignment and purity of CNTs in the fibers.

\subsubsection{Electrochemical Characterization of Dopamine Detection at CNT Fiber Microelectrodes}

To investigate the electrochemical performance, the response to $1 \mu \mathrm{M}$ dopamine was tested using FSCV with a triangle waveform from -0.4 to $1.3 \mathrm{~V}$ and back at $400 \mathrm{~V} / \mathrm{s}$ and a scan repetition frequency of $10 \mathrm{~Hz}$. Figures 5.2A-C show the background subtracted cyclic voltammograms (CVs) of $1 \mu \mathrm{M}$ dopamine at disk CA/CNT, PEI/CNT fiber, and CNT yarn microelectrodes with a similar diameter $(\sim 20 \mu \mathrm{m})$. The CNT yarn microelectrode has the largest oxidation and reduction currents as well as the smallest difference in oxidation and reduction peak potential $\left(\triangle \mathrm{E}_{\mathrm{p}}\right)($ Fig. $5.2 \mathrm{C})$. The current for the $\mathrm{CA} / \mathrm{CNT}$ and PEI/CNT fiber microelectrodes is similar, but the $\triangle \mathrm{E}_{\mathrm{p}}$ is smaller at CA/CNT fibers. 
To compare the electrochemical performance of microelectrodes, dopamine currents were corrected for surface area (based on their capacitive charging currents). As shown in Table 5.2, the current density at CNT yarn is $290 \pm 65 \mathrm{pA} / \mu \mathrm{m}^{2}$, which is significantly larger than the current density at PEI/CNT fiber (unpaired t-test, $p \leq 0.05$ ), but not statistically different with CA/CNT fiber (unpaired t-test, $p=0.2748$ ). The current density of CFMEs is $135 \pm 24 \mathrm{pA} / \mu \mathrm{m}^{2},{ }^{9}$ smaller than the CA/CNT fiber and CNT yarn.

Table 5.2: Comparison of Electrochemical Dopamine Detection

\begin{tabular}{|c|c|c|}
\hline & $\begin{array}{c}\text { Current Density } \\
\left(\mathrm{pA} / \mu \mathrm{m}^{2}\right)\end{array}$ & $\Delta \mathrm{E}_{\mathrm{p}} / \mathrm{V}$ \\
\hline CA/CNT Fiber & $210 \pm 31$ & $0.89 \pm 0.02$ \\
\hline PEI/CNT Fiber & $122 \pm 23$ & $1.04 \pm 0.02$ \\
\hline CNT Yarn & $290 \pm 65$ & $0.68 \pm 0.02$ \\
\hline
\end{tabular}

All $n=5$, Errors are standard error of mean

The $\Delta \mathrm{E}_{\mathrm{p}}$ values are significantly lower at CNT yarn than CA/CNT fiber or PEI/CNT fiber microelectrodes (Table 5.2, unpaired t-test, $\mathrm{p} \leq 0.0001$ for both comparisons). The $\Delta \mathrm{E}_{\mathrm{p}}$ value at CA/CNT fiber microelectrodes is significantly smaller than PEI/CNT fibers (unpaired t-test, $p \leq$ 0.01). The smaller $\Delta \mathrm{E}_{\mathrm{p}}$ might be caused by differing double-layer capacitances, uncompensated resistance, or ohmic drop. ${ }^{5}$ However, because both the electrolyte and the size of the electrodes are similar, ohmic drop is an unlikely cause. Therefore, the smaller $\Delta \mathrm{E}_{\mathrm{p}}$ value indicates faster electron transfer kinetics, with the fastest electron transfer rates observed in order of: CNT yarn > CA/CNT fiber > PEI/CNT fiber.

Figure 5.2D-F shows the current versus time curves in the response to a bolus of dopamine. CA/CNT fiber microelectrodes have a current versus time curve (Fig. 5.2D) similar to 
conventionally used CFMEs, with its time response performance falling between CNT yarn and $\mathrm{PEI} / \mathrm{CNT}$ fiber microelectrodes. The time response at PEI/CNT fiber microelectrodes is slow (Fig. 5.2E), the signal does not plateau, and does not go back to baseline. The CNT yarn microelectrode has a fast temporal response, as evidenced by the square shaped curve (Fig. $5.2 \mathrm{~F}$ ) with rapid signal rise and fall.

Background Subtracted CV Current-time Trace
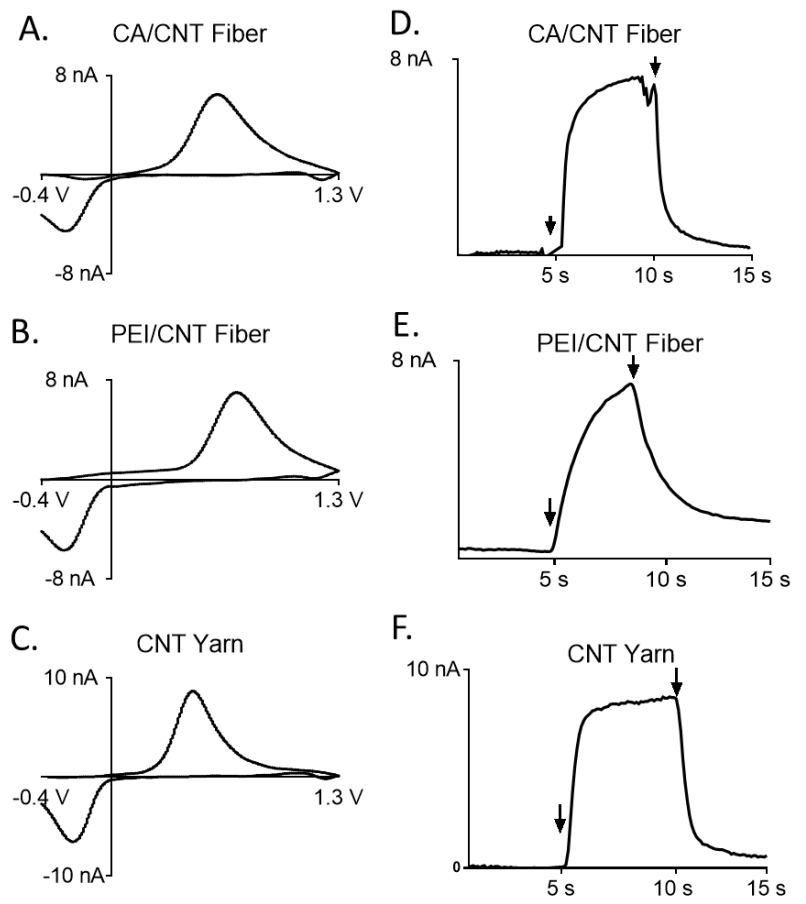

Figure 5.2: Electrochemical response to $1 \mu \mathrm{M}$ dopamine with a waveform of $-0.4 \mathrm{~V}$ to $1.3 \mathrm{~V}$ and back at $400 \mathrm{~V} / \mathrm{s}, 10 \mathrm{~Hz}$. (Left) Background subtracted cyclic voltammograms at (A) CA/CNT fiber, (B) PEI/CNT fiber, and (C) CNT yarn microelectrodes. (Right) Current versus time trace plot for the same electrodes measured at $0.6 \mathrm{~V}$ in flow injection experiment. The first arrow indicates the injection of dopamine bolus and the second arrow indicates the switch back to buffer.

\subsubsection{Electrochemical Characterization of Other Neurochemicals at CNT Fiber Microelectrodes}

Because many electroactive substances coexist in the brain, the selectivity of a sensor for neurotransmitters is another important factor to consider when selecting microelectrodes. We tested the electrochemical performance of different CNT fiber microelectrodes toward the 
detection of neurochemicals including uric acid, ascorbic acid, and serotonin. Uric acid (UA) acts as an antioxidant in cerebrospinal fluid and is an anion at physiological $\mathrm{pH} .{ }^{31}$ Ascorbic acid (AA), also an anion at physiological $\mathrm{pH}$, is one of the most abundant, low molecular weight antioxidants in the central nervous system. ${ }^{32} \mathrm{UA}$ and $\mathrm{AA}$ are common interferences for in vivo dopamine detection, and the extracellular levels of $\mathrm{UA}^{33}$ and $\mathrm{AA}^{34,35}$ are several orders of magnitude higher than dopamine in the brain. Serotonin is a cationic indolamine neurotransmitter with a similar oxidation potential to dopamine. ${ }^{36}$

Table 5.3: Average Oxidation Current Density for Different Neurochemicals at CA/CNT Fiber, PEI/CNT Fiber, and CNT Yarn Microelectrodes

\begin{tabular}{|c|c|c|c|}
\hline $\begin{array}{c}\text { Current Density } \\
\mathrm{pA} / \mu \mathrm{m}^{2}\end{array}$ & Uric Acid $(20 \mu \mathrm{M})$ & Ascorbic Acid $(200 \mu \mathrm{M})$ & Serotonin $(1 \mu \mathrm{M})$ \\
\hline CA/CNT Fiber & $182 \pm 45$ & $270 \pm 90$ & $34 \pm 9$ \\
\hline PEI/CNT Fiber & $122 \pm 36$ & $279 \pm 32$ & $23 \pm 8$ \\
\hline CNT Yarn & $1115 \pm 74$ & $2110 \pm 670$ & $1723 \pm 528$ \\
\hline
\end{tabular}

All $n=4$. Values are mean \pm standard error of mean

Figure 5.3A shows CVs for $20 \mu \mathrm{M} U A, 200 \mu \mathrm{M} \mathrm{AA}$, and $1 \mu \mathrm{M}$ serotonin at the same CA/CNT fiber, PEI/CNT fiber, or CNT yarn microelectrode. Table 5.3 gives the current density for different analytes at each electrode. The bar graphs (Fig. 5.3B) compare the ratio of oxidation currents for each neurotransmitter (20 $\mu \mathrm{M}$ UA, $200 \mu \mathrm{M}$ AA, or $1 \mu \mathrm{M}$ serotonin) to that of dopamine (DA, $1 \mu \mathrm{M})$. As shown in Figure 5.3B, the UA: DA signal ratio at CA/CNT and PEI/CNT fibers microelectrodes is significantly smaller than at CNT yarn microelectrodes (unpaired t-test, $p \leq$ 0.05 for both comparisons). Moreover, the AA: DA signal ratio is significantly smaller at CA/CNT fibers than CNT yarns (unpaired t-test, $p<0.05$ ). The CA/CNT fiber has the lowest UA and AA to DA signal ratios, indicating it has the highest selectivity for dopamine compared to UA and AA. 
Serotonin is positively charged at physiological pH; however, it is also known to foul the electrode surface when applying the waveform used here. ${ }^{37}$ At CA/CNT fibers, there is an extra peak found at $0.3-0.4 \mathrm{~V}$, which might be caused by polymerization of serotonin and indicative of fouling. The extra peak is also visible at PEI/CNT fibers, but much smaller than at CA/CNT fiber microelectrodes. In contrast, CNT yarn microelectrodes have a sharp, single oxidation peak for serotonin, which would give CNT yarn good serotonin anti-fouling property. The ratios of currents for serotonin to dopamine as well as the serotonin current densities are similar at CA/CNT and PEI/CNT fiber microelectrodes (Fig. 5.3B, unpaired $t$ test, $p=0.8463, n=4$, Table 5.3). In comparison, the serotonin: DA current ratio and the serotonin current density at CNT yarn microelectrodes are significantly larger than at CA/CNT and PEI/CNT fibers (unpaired t-test, $p \leq$ 0.0001 for both ratio and current density comparisons). PEI/CNT fiber are resistant to surface fouling by serotonin ${ }^{5}$, but the CNT yarn has dramatically higher sensitivity to serotonin compared to PEI/CNT and CA/CNT fiber microelectrodes. 


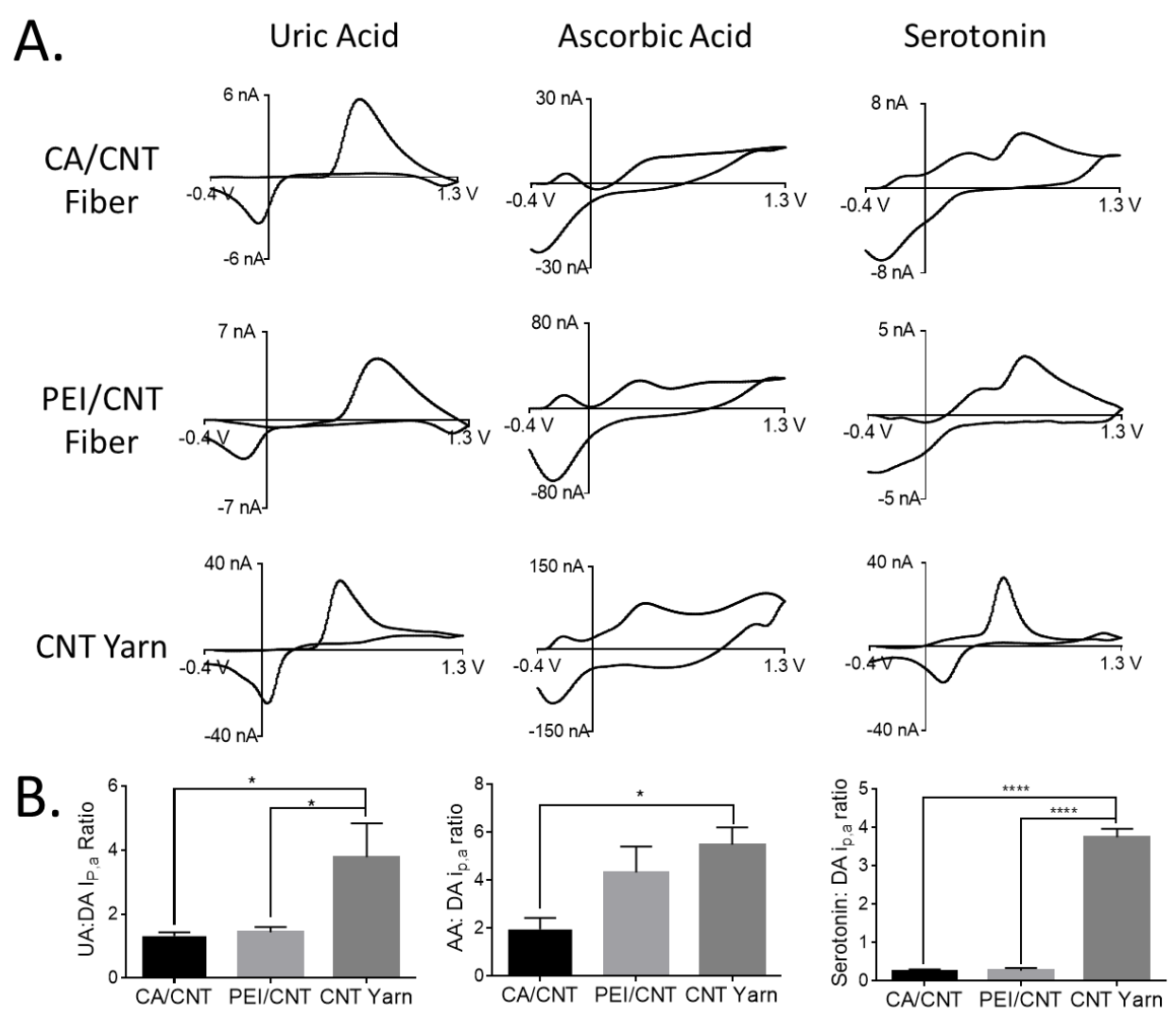

Figure 5.3: Detection of different neurochemicals at CNT fiber microelectrodes. (A) Detection of $20 \mu \mathrm{M}$ uric acid, $200 \mu \mathrm{M}$ ascorbic acid, and $1 \mu \mathrm{M}$ serotonin in PBS buffer at a CA/CNT fiber (top), PEI/CNT fiber (middle), or CNT yarn (bottom) microelectrode. (B) Bar graphs show the ratio of oxidation current for $\mathrm{UA}, \mathrm{AA}$, or serotonin compared to the oxidation current of dopamine $(\mathrm{n}=4$ each). ${ }^{*} p<0.05$ and ${ }^{* * *} p<0.0001$

\subsubsection{Dopamine Sensitivity at Rapid Scan Repetition Frequency}

The typical FSCV triangle waveform with a scan rate of $400 \mathrm{~V} / \mathrm{s}$ takes $8.5 \mathrm{~ms}$. The time between waveforms is $91.5 \mathrm{~ms}$ with a scan repetition frequency of $10 \mathrm{~Hz}$ but only $1.5 \mathrm{~ms}$ when the scan repetition frequency increases to $100 \mathrm{~Hz}$. The redox reaction of dopamine at the carbon based sensor surface is an adsorption-controlled process so the dopamine signal drops with decreasing time for dopamine adsorption at CFMEs. ${ }^{25,38}$ The loss of sensitivity with rapid scan repetition frequency at carbon fibers limits the temporal resolution of the measurement. Therefore, 
electrode materials which have a current that is independent of the scan repetition rate would be better able to monitor rapid reactions and follow the kinetics of neurotransmission. ${ }^{23}$

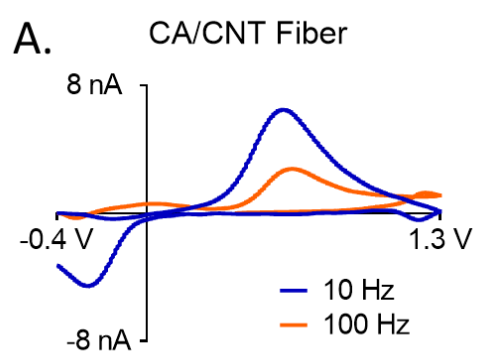

CNT Yarn

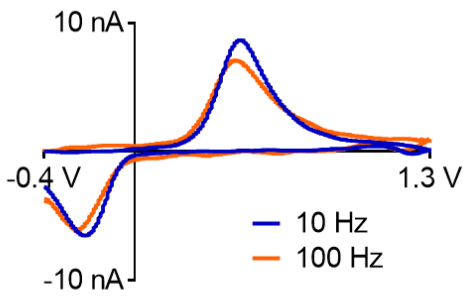

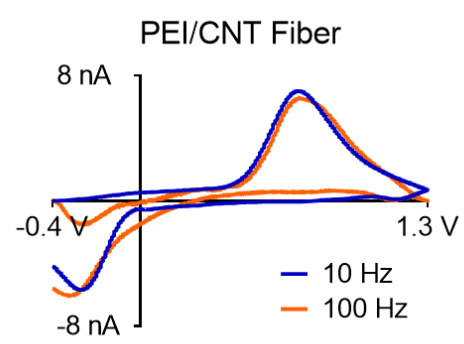

Carbon Fiber

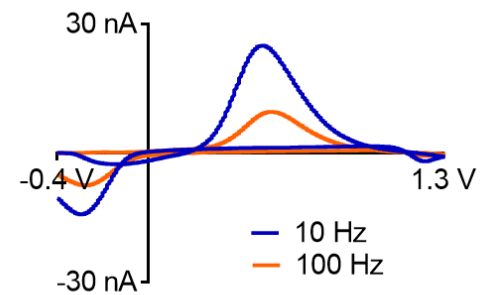

B.

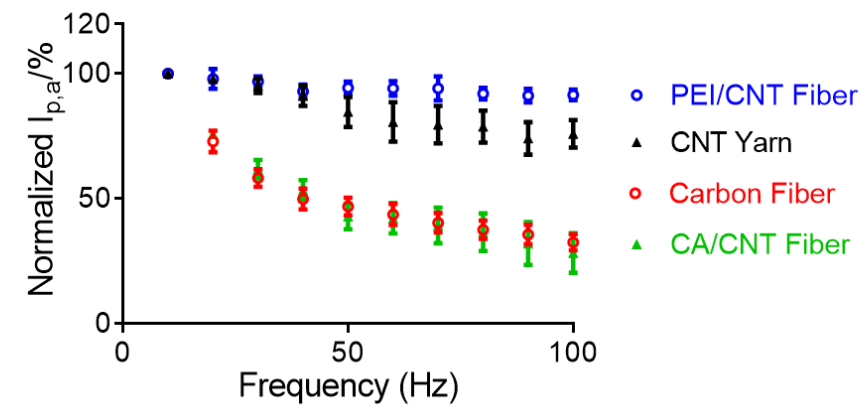

Figure 5.4: Effect of scan repetition frequency for $1 \mu \mathrm{M}$ dopamine detection. (A) Example CVs of $1 \mu \mathrm{M}$ dopamine at the scan repetition frequency of $10 \mathrm{~Hz}$ (blue line) and $100 \mathrm{~Hz}$ (orange line), with -0.4 to $1.3 \mathrm{~V}$ waveform. (B) Peak oxidation current at PEI/CNT fiber microelectrodes (blue circle, $n=4$ ), CNT yarn microelectrodes (black triangle, $n=5$ ), carbon fiber microelectrodes, (red circle, $n=5$ ), and CA/CNT fiber microelectrodes (green triangle, $n=4$ ), from top to bottom. Data is normalized to dopamine oxidation signal at different microelectrodes with scan repetition frequency of $10 \mathrm{~Hz}$.

To study the scan repetition frequency dependence, the current for $1 \mu \mathrm{M}$ dopamine was measured with scan repetition frequencies from 10 to $100 \mathrm{~Hz}$ (Figure 5.4A). The oxidation current is relatively stable with increasing scan frequency at PEI/CNT and CNT yarn microelectrodes, but decreases dramatically at CA/CNT fiber and CFMEs. Moreover, the oxidation/reduction peak ratio is larger at PEI/CNT fiber and CNT yarn than those at carbon fiber and CA/CNT fiber at both 10 
and $100 \mathrm{~Hz}$. Figure 5.4B shows a significant effect of electrode type on dopamine current with different scan repetition frequencies (one-way ANOVA, $p<0.0001, n=4$ ). The oxidation current drops dramatically at CFMEs, with approximately $67 \%$ loss at $100 \mathrm{~Hz}$ compared to $10 \mathrm{~Hz}$, and the current at CA/CNT fiber microelectrodes dropped similarly, $72 \%$ at $100 \mathrm{~Hz}$ (one-way ANOVA Bonferroni post-test, $p=0.9561$ ). The oxidation currents of dopamine at CNT yarn and PEI/CNT fiber microelectrodes dropped approximately $24 \%$ and $8.4 \%$ at $100 \mathrm{~Hz}$, respectively, a significantly smaller drop than CA/CNT fibers (one-way ANOVA. Bonferroni post-test, $p \leq 0.001$ and $p \leq 0.0001$, respectively) or CFMEs ( $p \leq 0.001$ and $p \leq 0.0001$, respectively). The results are consistent with our previous work that the current at CNTYMEs ${ }^{23}$, laser treated CNTYMEs ${ }^{39}$, and $\mathrm{PEI} / \mathrm{CNT}$ fiber ${ }^{5}$ retain high sensitivity at rapid scan repetition frequency.

\subsubsection{Correlation of Electrochemical Performance and Surface Properties}

Surface properties of CNT fibers produced by different protocols have substantial influences on the electrochemical performance for the detection of neurotransmitters. The better aligned CNTs in CNT yarns and CA/CNT fibers, compared to PEI/CNT fibers, provide higher overall conductivity and better sensitivity for dopamine detection (Table 5.2). The extra current density observed at CNT yarns over CA/CNTs is attributed to the relatively higher oxygen content and less residue on CNT yarn surface (Table 5.1). CNT yarns have abundant oxygen containing functional groups, such as quinones, hydroxyls, and carboxylic acids, which are known to be negatively charged at physiological $\mathrm{pH}$ and have electrostatic interactions with positively charged neurotransmitters, such as dopamine. ${ }^{40}$ The $\Delta \mathrm{E}_{\mathrm{p}}$, a measure of electron transfer kinetics, followed the same order as CNT alignment: CNT yarns > CA/CNT fibers > PEI/CNT fibers. In addition, the faster electron transfer rate observed at CA/CNTs compared to PEI/CNT fiber microelectrodes might be caused less residue and higher conductivity. 
The CA/CNT fiber was applied as a biomolecule microelectrode material for the first time. It provides high selectivity to dopamine over UA and AA because of the negative charge carried by the acid-doping at the fiber surface, which makes CA/CNT fibers a good microelectrode material for dopamine detection in the presence of large amount of interferences. CNT yarn microelectrodes have dramatically higher serotonin sensitivity compared to PEI/CNT and CA/CNT fiber microelectrodes. The abundant oxygen containing functional groups provide electroactive sites with negative charges, which are good for adsorption of serotonin, a cation at physiological $\mathrm{pH}$, similar to dopamine.

A.

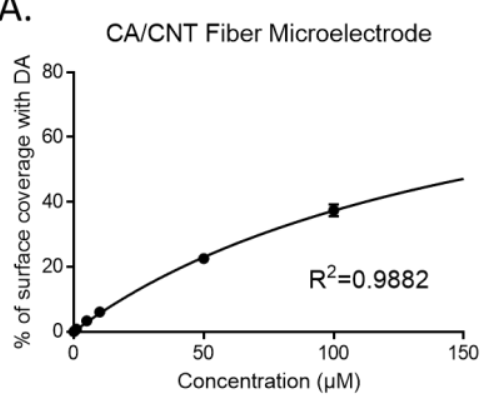

D.

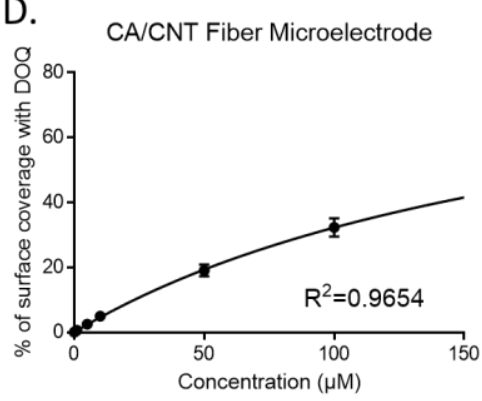

B.

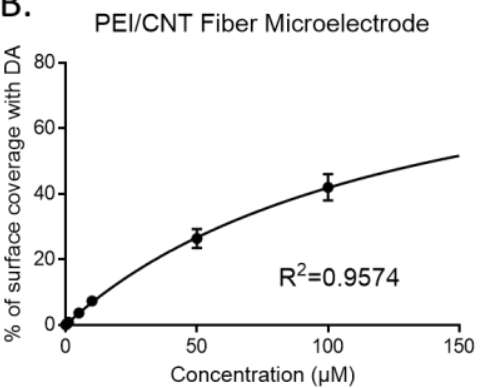

E.

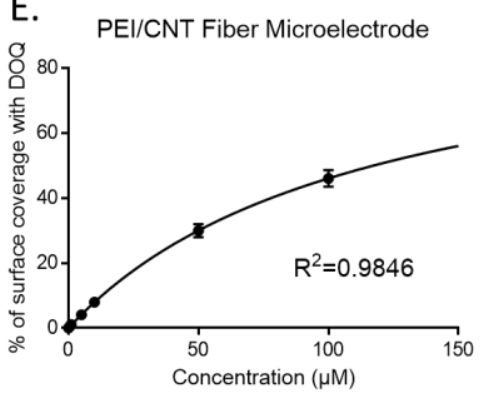

C.

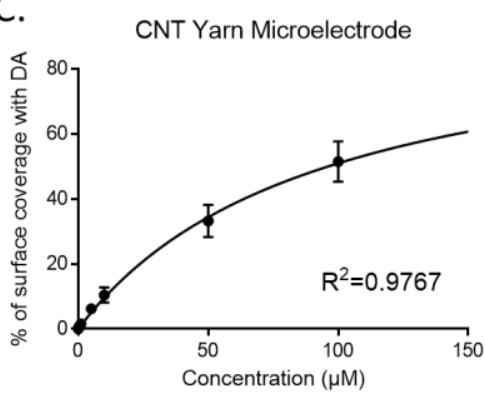

F.

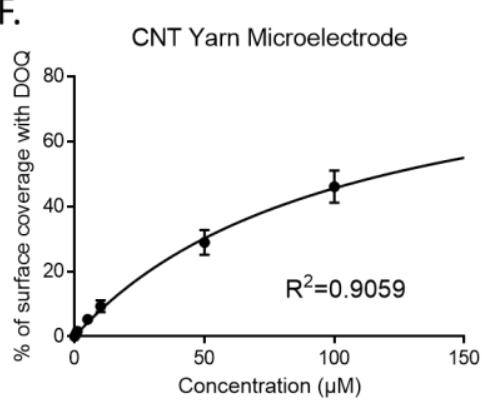

Figure 5.5: Langmuir isotherm plots. The $y$-axis is fractional surface coverage and $x$-axis is $C_{D A}$ for dopamine. The fitting is modeled with $\frac{\Gamma_{D A}}{\Gamma_{S}}=\frac{\beta_{D A} \cdot \gamma_{D A} \cdot C_{D A}}{1+\beta_{D A} \cdot \gamma_{D A} \cdot C_{D A}}$, where $\gamma_{D A}=0.63$, and a $\beta$ is determined. An equilibrium value based on anodic current, $\beta_{D A}$, is fit for $(A) C A / C N T$ fiber $((10 \pm$ 1) $\left.\times 10^{3}\right)$, (B) PEI/CNT fiber $\left((14 \pm 1) \times 10^{3}\right)$, and (C) CNT yarn microelectrodes $\left((19 \pm 3) \times 10^{3}\right)$. $\beta_{\text {DoQ }}$ based on cathodic current is fit for (D) CA/CNT fiber $\left((8 \pm 1) \times 10^{3}\right)$, (E) PEI/CNT fiber ((14 \pm 1) $\left.\times 10^{3}\right)$, and $(F)$ CNT yarn microelectrodes $\left((19 \pm 4) \times 10^{3}\right) . n=5$ per electrode; error bar is standard error of mean.

The frequency independent property of CNT yarn and PEI/CNT fiber microelectrodes allows highly sensitive detection of dopamine at scan repetition frequency of $100 \mathrm{~Hz}$, which is one 
magnitude faster than the conventionally applied frequency. To understand adsorption strength, we applied a Langmuir adsorption isotherm to model the adsorption and desorption of dopamine as well as the oxidation product of dopamine, dopamine-o-quinone (DOQ). ${ }^{9}$ Plotting percent surface coverage versus concentration for the anodic peaks (Fig. $5.5 \mathrm{~A}-\mathrm{C}$ ) and cathodic peaks (Fig. 5.5 D-F) gives information about dopamine and DOQ adsorption/desorption, respectively. The adsorption/desorption equilibrium constants for both dopamine $\left(\beta_{\mathrm{DA}}\right)$ and DOQ $\left(\beta_{\mathrm{DOQ}}\right)$ at CNT yarn microelectrodes are larger than those at CA/CNT fiber and PEI/CNT fiber microelectrodes (Table 5.4, one-way ANOVA, Brown-Forsythe test, $p<0.01$, and $p<0.0001$ for $\beta_{\mathrm{DA}}$ and $\beta_{\mathrm{DOQ}}$, respectively), indicating dopamine and DOQ adsorption is stronger on the CNT yarn surface than the other two CNT fibers. The strong adsorption is likely due to the abundant oxygen containing functional groups and fewer impurities (both polymer residues and iron catalyst) at CNT yarns. The ratio of $\beta_{\mathrm{DA}}$ to $\beta_{\mathrm{DOQ}}$ is used to compare the adsorption equilibria differences between dopamine and DOQ. A ratio larger than 1 means that dopamine adsorption is stronger than $\mathrm{DOQ}$ and that DOQ has a greater tendency to fall off, leading to lower reversibility. In comparison, a ratio of 1 indicates dopamine and DOQ have similar adsorption strength, so more DOQ will remain adsorbed and be reduced back to dopamine, amplifying dopamine detection on the next scan and giving the material a frequency independent property. The $\beta_{\mathrm{DA}} / \beta_{\mathrm{DOQ}}$ ratio is about 1 at PEI/CNT fibers and CNT yarn microelectrodes, which matches well with the better reversibility of the dopamine signal at these electrodes (Fig. 5.2A-C, Fig. 5.4A). The ratio of equilibrium constants for CA/CNT fiber microelectrodes is greater than 1 and significantly larger than PEI/CNT fiber (unpaired t-test, $p \leq 0.01$ ) or CNT yarn microelectrodes (unpaired t-test, $p \leq 0.05$ ), but similar to CFMEs (with ratio of $1.23 \pm 0.04)^{9}$. Thus, DOQ is more likely to desorb from the surface of CA/CNT fibers, leading to smaller reduction peak and less reversible reaction. These data agree with previous modeling of CNT yarn and CNT-metal microelectrodes, which showed the similar $\beta_{\text {DA }}$ to $\beta_{\text {DOQ }}$ values lead to frequency independent currents. ${ }^{9,23}$ 
Table 5.4: Average Equilibrium Constants ( $\beta$ ) for Dopamine and Dopamine-o-quinone

\begin{tabular}{|c|c|c|c|}
\hline Material & $\beta_{\mathrm{DA}}\left(\times 10^{3}\right)$ & $\beta_{\mathrm{DOQ}}\left(\times 10^{3}\right)$ & $\beta_{\mathrm{DA}} / \beta_{\mathrm{DOQ}}$ \\
\hline CA/CNT Fiber & $10 \pm 1$ & $8 \pm 1$ & $1.27 \pm 0.07$ \\
\hline PEI/CNT Fiber & $14 \pm 1$ & $14 \pm 1$ & $0.97 \pm 0.04$ \\
\hline CNT Yarn & $19 \pm 3$ & $19 \pm 4$ & $1.08 \pm 0.09$ \\
\hline
\end{tabular}

All $n=4$, Errors are standard error of mean.

Surface roughness also influences the electrochemical properties. ${ }^{39}$ Dopamine and DOQ are more likely to get trapped in the rough surface of CNT yarn and PEI/CNT fiber (Fig. 5.1E and $5.1 F)$, leading to better reversibility. Surface roughness also influences the scan frequency dependence. At smoother surfaces, such as the CA/CNT electrode, when DOQ desorbs, it can easily diffuse away from the electrode surface. At the rough microelectrode surfaces of CNT yarns or PEI/CNT fibers, the response would be similar to that in a thin layer cell, where the DOQ would remain close to the surface even if it desorbs and thus could easily adsorb again. The CNT yarn (with mean roughness depth of $1910 \pm 190 \mathrm{~nm})^{39}$ does not trap the dopamine long enough to impair the current-vs-time curve. However, the deep corrugations at PEI/CNT fiber surface (0.5 $1 \mu \mathrm{m}^{15}$, Fig. $\left.5.1 \mathrm{E}\right)$ influence the temporal response remarkably, trapping dopamine and slowing the diffusion to and from the surface, as indicated by the slow rise and fall of the curve (Fig. 5.2E). Therefore, CNT yarn microelectrodes provide high sensitivity at rapid scan repetition frequency while maintaining a fast temporal response. 


\subsection{Conclusions}

Surface properties of CNT fibers produced by different protocols have dramatic effects on the electrochemical performance including sensitivity, selectivity, and response to FSCV frequency. CNT yarn microelectrodes have the highest dopamine and serotonin sensitivity because of the well-aligned, high purity CNTs, and abundant oxygen containing functional groups at the surface. The CA/CNT fiber, which was applied as electrode material for biomolecule detection for the first time, provides high selectivity to dopamine over UA and AA because of the negatively charged surface. CNT yarn and PEI/CNT fiber microelectrodes have an electrochemical response to dopamine that is reversible and scan frequency independent due to the surface roughness that allows dopamine to get trapped in the crevices, leading to thin-layer cell conditions. However, the larger surface roughness on the PEI/CNT fiber surface is detrimental to fast temporal response. The studies result in a better understanding of how surface properties influence the electrochemical properties of neurotransmitter detection with FSCV. Thus, surface properties can be tuned in the future and used to predict which carbon nanomaterial-based microelectrodes will be best for detection of different neurotransmitters. 
Yang| 155

\subsection{References}

(1) Yang, C.; Denno, M. E.; Pyakurel, P.; Venton, B. J. Recent Trends in Carbon NanomaterialBased Electrochemical Sensors for Biomolecules: A Review. Anal. Chim. Acta 2015, 887, 17-37.

(2) Swamy, B. E. K.; Venton, B. J. Carbon Nanotube-Modified Microelectrodes for Simultaneous Detection of Dopamine and Serotonin in Vivo. Analyst 2007, 132 (9), 876884.

(3) Takmakov, P.; Zachek, M. K.; Keithley, R. B.; Walsh, P. L.; Donley, C.; McCarty, G. S.; Wightman, R. M. Carbon Microelectrodes with a Renewable Surface. Anal. Chem. 2010, 82 (5), 2020-2028.

(4) Xiao, N.; Venton, B. J. Rapid, Sensitive Detection of Neurotransmitters at Microelectrodes Modified with Self-Assembled SWCNT Forests. Anal. Chem. 2012, 84 (18), 7816-7822.

(5) Zestos, A. G.; Jacobs, C. B.; Trikantzopoulos, E.; Ross, A. E.; Venton, B. J. Polyethylenimine Carbon Nanotube Fiber Electrodes for Enhanced Detection of Neurotransmitters. Anal. Chem. 2014, 86, 8568-8575.

(6) Zestos, A. G.; Yang, C.; Jacobs, C. B.; Hensley, D.; Venton, B. J. Carbon Nanospikes Grown on Metal Wires as Microelectrode Sensors for Dopamine. Analyst 2015, 140 (21), 7283-7292.

(7) Jacobs, C. B.; Vickrey, T. L.; Venton, B. J. Functional Groups Modulate the Sensitivity and Electron Transfer Kinetics of Neurochemicals at Carbon Nanotube Modified Microelectrodes. Analyst 2011, 136 (17), 3557.

(8) Xiang, L.; Yu, P.; Hao, J.; Zhang, M.; Zhu, L.; Dai, L.; Mao, L. Vertically Aligned Carbon Nanotube-Sheathed Carbon Fibers as Pristine Microelectrodes for Selective Monitoring of Ascorbate in Vivo. Anal. Chem. 2014, 86 (8), 3909-3914.

(9) Yang, C.; Jacobs, C. B.; Nguyen, M. D.; Ganesana, M.; Zestos, A. G.; Ivanov, I. N.; Puretzky, A. A.; Rouleau, C. M.; Geohegan, D. B.; Venton, B. J. Carbon Nanotubes Grown on Metal Microelectrodes for the Detection of Dopamine. Anal. Chem. 2016, 88 (1), 645652.

(10) Spitalsky, Z.; Tasis, D.; Papagelis, K.; Galiotis, C. Carbon Nanotube-Polymer Composites: Chemistry, Processing, Mechanical and Electrical Properties. Prog. Polym. Sci. 2010, 35 (3), 357-401.

(11) Lu, W.; Zu, M.; Byun, J. H.; Kim, B. S.; Chou, T. W. State of the Art of Carbon Nanotube Fibers: Opportunities and Challenges. Adv. Mater. 2012, 24 (14), 1805-1833.

(12) Li, Z.; Liu, Z.; Sun, H.; Gao, C. Superstructured Assembly of Nanocarbons: Fullerenes, Nanotubes, and Graphene. Chem. Rev. 2015, 115 (15), 7046-7117.

(13) Behabtu, N.; Young, C. C.; Tsentalovich, D. E.; Kleinerman, O.; Wang, X.; Ma, A. W. K.; Bengio, E. A.; ter Waarbeek, R. F.; de Jong, J. J.; Hoogerwerf, R. E.; Fairchild, S. B.; Ferguson, J. B.; Maruyama, B.; Kono, J.; Talmon, Y.; Cohen, Y.; Otto, M. J.; Pasquali, M. Strong, Light, Multifunctional Fibers of Carbon Nanotubes with Ultrahigh Conductivity. Science (80-. ). 2013, 339 (6116), 182-186.

(14) Vigolo, B.; Pénicaud, A.; Coulon, C.; Sauder, C.; Pailler, R.; Journet, C.; Bernier, P.; Poulin, P. Macroscopic Fibers and Ribbons of Oriented Carbon Nanotubes. Science (80-. ). 2000, 
290 (5495), 1331-1334.

(15) Muñoz, E.; Suh, D. S.; Collins, S.; Selvidge, M.; Dalton, A. B.; Kim, B. G.; Razal, J. M.; Ussery, G.; Rinzler, A. G.; Martínez, M. T.; Baughman, R. H. Highly Conducting Carbon Nanotube/polyethyleneimine Composite Fibers. Adv. Mater. 2005, 17 (8), 1064-1067.

(16) Ericson, L. M.; Fan, H.; Peng, H.; Davis, V. A.; Zhou, W.; Sulpizio, J.; Wang, Y.; Booker, R.; Vavro, J.; Guthy, C.; Parra-Vasquez, A. N. G.; Kim, M. J.; Ramesh, S.; Saini, R. K.; Kittrell, C.; Lavin, G.; Schmidt, H.; Adams, W. W.; Billups, W. E.; Pasquali, M.; Hwang, W.F.; Hauge, R. H.; Fischer, J. E.; Smalley, R. E. Macroscopic, Neat, Single-Walled Carbon Nanotube Fibers. Science (80-. ). 2004, 305 (5689), 1447-1450.

(17) Davis, V. a; Parra-Vasquez, a N. G.; Green, M. J.; Rai, P. K.; Behabtu, N.; Prieto, V.; Booker, R. D.; Schmidt, J.; Kesselman, E.; Zhou, W.; Fan, H.; Adams, W. W.; Hauge, R. H.; Fischer, J. E.; Cohen, Y.; Talmon, Y.; Smalley, R. E.; Pasquali, M. True Solutions of Single-Walled Carbon Nanotubes for Assembly into Macroscopic Materials. Nat. Nanotechnol. 2009, 4 (12), 830-834.

(18) Fujigaya, T.; Nakashima, N. Methodology for Homogeneous Dispersion of Single-Walled Carbon Nanotubes by Physical Modification. Polym. J. 2008, 40 (7), 577-589.

(19) Koziol, K.; Vilatela, J.; Moisala, A.; Motta, M.; Cunniff, P.; Sennett, M.; Windle, A. HighPerformance Carbon Nanotube Fiber. Science (80-. ). 2007, 318 (5858), 1892-1895.

(20) Zhang, M.; Atkinson, K. R.; Baughman, R. H. Multifunctional Carbon Nanotube Yarns by Downsizing an Ancient Technology. Science 2004, 306 (5700), 1358-1361.

(21) Mayhew, E.; Prakash, V. Thermal Conductivity of High Performance Carbon Nanotube Yarn-like Fibers. J. Appl. Phys. 2014, 115 (17), 174306.

(22) Jiang, K.; Li, Q.; Fan, S. Nanotechnology: Spinning Continuous Carbon Nanotube Yarns. Nature 2002, 419 (6909), 801.

(23) Jacobs, C. B.; Ivanov, I. N.; Nguyen, M. D.; Zestos, A. G.; Venton, B. J. High Temporal Resolution Measurements of Dopamine with Carbon Nanotube Yarn Microelectrodes. Anal. Chem. 2014, 86 (12), 5721-5727.

(24) Schmidt, A. C.; Wang, X.; Zhu, Y.; Sombers, L. a. Carbon Nanotube Yarn Electrodes for Enhanced Detection of Neurotransmitter Dynamics in Live Brain Tissue. ACS Nano 2013, 7864-7873.

(25) Bath, B. D.; Michael, D. J.; Trafton, B. J.; Joseph, J. D.; Runnels, P. L.; Wightman, R. M. Subsecond Adsorption and Desorption of Dopamine at Carbon-Fiber Microelectrodes. Anal. Chem. 2000, 72 (24), 5994-6002.

(26) Graham, D. The Characterization of Physical Adsorption Systems. I. The Equilibrium Function and Standard Free Energy of Adsorption. J. Phys. Chem. 1953, 57 (7), 665-669.

(27) Liu, Y. Is the Free Energy Change of Adsorption Correctly Calculated? J. Chem. Eng. Data 2009, 54 (7), 1981-1985.

(28) Archer, D. G.; Wang, P. The Dielectric Constant of Water and Debye-Hückel Limiting Law Slopes. J. Phys. Chem. Ref. Data 1990, 19 (2), 371.

(29) Mortimer, R. G. Physical Chemistry, Third Edit.; Academic Press: Memphis, TN, USA, 2008.

(30) Miao, M. Electrical Conductivity of Pure Carbon Nanotube Yarns. Carbon N. Y. 2011, 49 
(12), 3755-3761.

(31) Amaro, S.; Urra, X.; Gómez-Choco, M.; Obach, V.; Cervera, Á.; Vargas, M.; Torres, F.; Rios, J.; Planas, A. M.; Chamorro, Á. Uric Acid Levels Are Relevant in Patients with Stroke Treated with Thrombolysis. Stroke 2011, 42 (SUPPL. 1), 28-33.

(32) Rice, M. .; Russo-Menna, I. Differential Compartmentalization of Brain Ascorbate and Glutathione between Neurons and Glia. Neuroscience 1997, 82 (4), 1213-1223.

(33) Dutt, V.; Mottola, H. Determination of Uric Acid at the Microgram Level by a Kinetic Procedure Based on a Pseudo-Induction Period. Anal. Chem. 1974, No. October, 17771781.

(34) Goldstein, D. S.; Holmes, C. Neuronal Source of Plasma Dopamine. Clin. Chem. 2008, 54 (11), 1864-1871.

(35) Tallaksen, C. M.; Bøhmer, T.; Bell, H. Concentrations of the Water-Soluble Vitamins Thiamin, Ascorbic Acid, and Folic Acid in Serum and Cerebrospinal Fluid of Healthy Individuals. Am. J. Clin. Nutr. 1992, 56 (3), 559-564.

(36) Baur, J. E.; Kristensen, E. W.; May, L. J.; Wiedemann, D. J.; Wightman, R. M. Fast-Scan Voltammetry of Biogenic Amines. Anal. Chem. 1988, 60 (13), 1268-1272.

(37) Jackson, B.; Dietz, S.; Wightman, R. Fast-Scan Cyclic Voltammetry of 5Hydroxytryptamine. Anal. Chem. 1995, No. 9, 1115-1120.

(38) Atcherley, C. W.; Laude, N. D.; Parent, K. L.; Heien, M. L. Fast-Scan Controlled-Adsorption Voltammetry for the Quantification of Absolute Concentrations and Adsorption Dynamics. Langmuir 2013, 29 (48), 14885-14892.

(39) Yang, C.; Trikantzopoulos, E.; Nguyen, M. D.; Jacobs, C. B.; Wang, Y.; Mahjouri-Samani, M.; Ivanov, I. N.; Venton, B. J. Laser Treated Carbon Nanotube Yarn Microelectrodes for Rapid and Sensitive Detection of Dopamine in Vivo. ACS Sensors 2016, 1 (5), 508-515.

(40) Roberts, J. G.; Moody, B. P.; McCarty, G. S.; Sombers, L. a. Specific Oxygen-Containing Functional Groups on the Carbon Surface Underlie an Enhanced Sensitivity to Dopamine at Electrochemically Pretreated Carbon Fiber Microelectrodes. Langmuir 2010, 26 (11), 9116-9122. 
Chapter 6: Novel Carbon-Fiber Microelectrode Batch Fabrication using a 3D-Printed Mold and Polyimide Resin 


\section{Abstract}

Glass insulated carbon-fiber microelectrodes (CFMEs) are standard tools for the measurement of neurotransmitters. However, electrodes are fabricated individually and the glass can shatter, limiting application in higher order mammals. Here, we developed a novel microelectrode batch fabrication method using a 3D-printed mold and polyimide resin insulating agent. The 3D-printed mold is low cost, customizable to change the electrode shape, and allows 40 electrodes to be made simultaneously. The polyimide resin is biocompatible, quick to cure, and does not adhere to the plastic mold. The electrodes were tested for the response to dopamine with fast-scan cyclic voltammetry both in vitro and in vivo and performed similarly to traditional glass-insulated electrodes, but with lower background currents. Thus, polyimide-insulated electrodes can be mass-produced using a 3D-printed mold and are an attractive alternative for making cheap, biocompatible microelectrodes. This paper was published in Analyst (Analyst, 2016, 141 (18), 5256-5260). 
Yang $\mid 160$

\subsection{Introduction}

Carbon-fiber microelectrodes (CFMEs) have served as the standard tool for detection of electroactive neurotransmitters in vivo. ${ }^{1}$ CFMEs have multiple advantages including biocompatibility, fast electron transfer kinetics for neurotransmitters, and good adsorption properties for biogenic amines. ${ }^{2,3}$ The traditional method for CFME fabrication involves threading an individual, cylindrical carbon fiber into a borosilicate glass capillary that is drawn to a sharp tip using a vertical capillary puller. ${ }^{4}$ However, the glass insulation method has several disadvantages..$^{5,6}$ First, glass-insulated CFMEs are fabricated one at a time and require a series of non-automated steps, making mass production difficult. Second, glass electrodes can potentially shatter in tissue, and so they are not permitted for use in higher order mammals. ${ }^{7}$ Therefore, new methods are desirable that reproducibly produce non-glass insulated CFMEs economically on a large scale.

An easy method to mass fabricate small objects reproducibly is to use a mold. Poly(dimethylsiloxane) (PDMS) molds have been made extensively using photolithography but the process requires a master to be made prior to mold production. ${ }^{8}$ Laser etching of materials, such as Teflon, has been used to design molds for microelectrode fabrication. ${ }^{5}$ However, photolithography and laser etching are restricted by a limited depth of focus and aspect ratio, ${ }^{9}$ and these methods require high cost instruments or long production times. 3D printing is an emerging technology that allows for rapid prototyping with high reproducibility and low cost. ${ }^{10}$ The method has the advantage that it can create almost any complex shape or geometric feature; 3D printers have reached extremely high resolution within microns and are constantly being improved. ${ }^{11,12}$ Thus, 3D printing provides an alternative method of mass-fabricating electrochemical devices, such as stainless steel electrodes, ${ }^{13}$ or electrochemical chips for direct biological measurements. ${ }^{14}$ However, the fabrication of electrochemical probes with micron diameters has not been reported with 3D printed devices. 
Making electrodes in a mold requires alternative insulations other than glass, which may be beneficial for designing electrodes that are biocompatible and shatter resistant. Epoxy resin has been used previously to completely insulate carbon fiber, ${ }^{8}$ and paraffin wax to seal glass capillary CFMEs. ${ }^{9}$ Polyimide-coated, fused silica capillaries have been developed as another method of insulating carbon-fibers for chronic tissue implantation. ${ }^{15}$ While these polyimide coated capillaries are sold as a commercial product, polyimide is a mechanically flexible insulator that can be poured into molds and manipulated into unique three-dimensional designs. ${ }^{16-18}$ Thus, polyimide has been used as a biocompatible insulator to provide an optimal implant environment and extend the longevity of the tissue-electrode interface..$^{16}$

In this work, we develop a 3D-printed mold for in vivo microelectrodes and use it to make polyimide-insulated CFMEs. The 3D-printed mold enables a rapid and low cost fabrication of the desired electrode geometry with high resolution features. These electrodes have a similar performance for dopamine detection to traditional glass-insulated electrodes, both in vitro and in vivo, and can be batch fabricated and customized to different shapes. 


\subsection{Experimental}

\subsubsection{D printed mold fabrication}

Molds were designed in Autodesk Inventor Professional 2014 Student Edition, converted to an .STL file, and subsequently printed by the Department of Mechanical Engineering at University of Virginia. The 3D printed polymer molds were manufactured on a Stratasys Connex 500 Model 1 Poly-Jet 3D printer (Stratasys Ltd., MN), which has 8 print heads with 96 nozzles per head. Water jets were used to remove the support. Rigid opaque black material (VeroBlackPlus RGD875, mainly acrylonitrile butadiene styrene, Stratasys) was used because of its suitability for rapid tooling with dimensional stability and fine detail. The heads heat up to $60^{\circ} \mathrm{C}$, and the $\mathrm{Z}$ axis resolution with this material was $30 \mu \mathrm{m}$. Electrodes were fabricated with carbon fibers $(7 \mu \mathrm{m}$ in diameter, T650, Cytec Engineering Materials, West Patterson, NJ). Polyimide sealing resin (Grace Davison Discovery Sciences, Deerfield, IL) was used to fill the mold and provide insulation.

\subsubsection{Electrochemical instrumentation}

Fast scan cyclic voltammetry (FSCV) was performed using a ChemClamp potentiostat (Dagan, Minneapolis, MN). The waveform was generated and the data was collected using a High Definition Cyclic Voltammetry (HDCV) breakout box, HDCV analysis software program (UNC Chemistry Department, Electronics Design Facility) and PCle-6363 computer interface cards (National Instruments, Austin, TX). Electrodes were backfilled with $1 \mathrm{M} \mathrm{KCl}$ and a silver wire was inserted to connect the electrode with the potentiostat headstage. A triangle waveform was applied to the electrode from a holding potential of $-0.4 \mathrm{~V}$ to $1.3 \mathrm{~V}$ and back at a scan rate of 400 V/s and a frequency of $10 \mathrm{~Hz}$. A silver-silver chloride wire was used as the reference electrode. Samples were tested using a flow injection analysis system as previously described. ${ }^{2}$ Buffer and samples were pumped through the flow cell at $2 \mathrm{~mL} / \mathrm{min}$ using a syringe pump (Harvard Apparatus, Holliston, MA). 


\subsubsection{Animals}

Male Sprague-Dawley rats (250-350 g) purchased from Charles River were housed in a vivarium and given food and water ab libitum. All experiments were approved by the Animal Care and Use Committee of the University of Virginia. The rat was anesthetized with urethane (1.5 $\mathrm{mg} / \mathrm{kg}$ i.p. $)$, the scalp shaved, and $0.25 \mathrm{~mL}$ bupivicaine $(0.25 \%$ solution) given subcutaneously. The working electrode was implanted in the caudate putamen (in mm from bregma: AP + 1.2, ML +2.0 , and $\mathrm{DV}-4.5$ to 5.0$)$, the stimulating electrode in the substantia nigra $(\mathrm{AP}-5.4, \mathrm{ML}+1.2$, and $\mathrm{DV}-7.5)$, and the $\mathrm{Ag} / \mathrm{AgCl}$ reference electrode in the contralateral side of the brain. The DV placement of the stimulating electrode was adjusted downward until a robust dopamine signal was measured. The polyimide-insulated carbon-fiber electrode was inserted into the brain and the FSCV waveform applied for $30 \mathrm{~min}$ to allow the electrode to stabilize. Stimulated release was electrically evoked using biphasic stimulation pulses (300 $\mu \mathrm{A}, 30-120$ pulses, $60 \mathrm{~Hz})$. 


\subsection{Results \& Discussion}

\subsubsection{Polyimide-Insulated Microelectrode Fabrication Using 3D-Printed Mold}

Polyimide-insulated microelectrodes were made using a 3D-printed mold. Figure 6.1A shows an example of a single channel in the mold. Our design used channels with sharp tips that are $150 \mu \mathrm{m}$ in diameter and $3 \mathrm{~mm}$ in length that connects to a tapered section that is $5 \mathrm{~mm}$ long, making the total polyimide section $8 \mathrm{~mm}$. This length is longer than the conventional penetration depth $(4.5-5 \mathrm{~mm})$ for the caudate putamen, but the length could be customized for other brain regions or applications. ${ }^{19,20}$ There is also a $15 \mathrm{~mm}$ section designed for microelectrode support, which is a cannula needle in this design. Cannula needles made of stainless steel are widely used for neuroscience studies and are safer for brain insertion compared to glass capillaries. ${ }^{21}$ A 23 gauge cannula needle was used as the connector, with inner diameter of $0.34 \mathrm{~mm}$ and outer diameter of $0.64 \mathrm{~mm}$, which is half the outer diameter of conventionally used glass capillaries (1.2 $\mathrm{mm})$. Figure 6.1B shows an image of an actual channel while Fig. 6.1C shows an electrode after fabrication. The whole mold design includes 40 electrodes per side, and the number of channels and the dimensions can be easily customized by changing the computer-aided design (CAD) parameters.
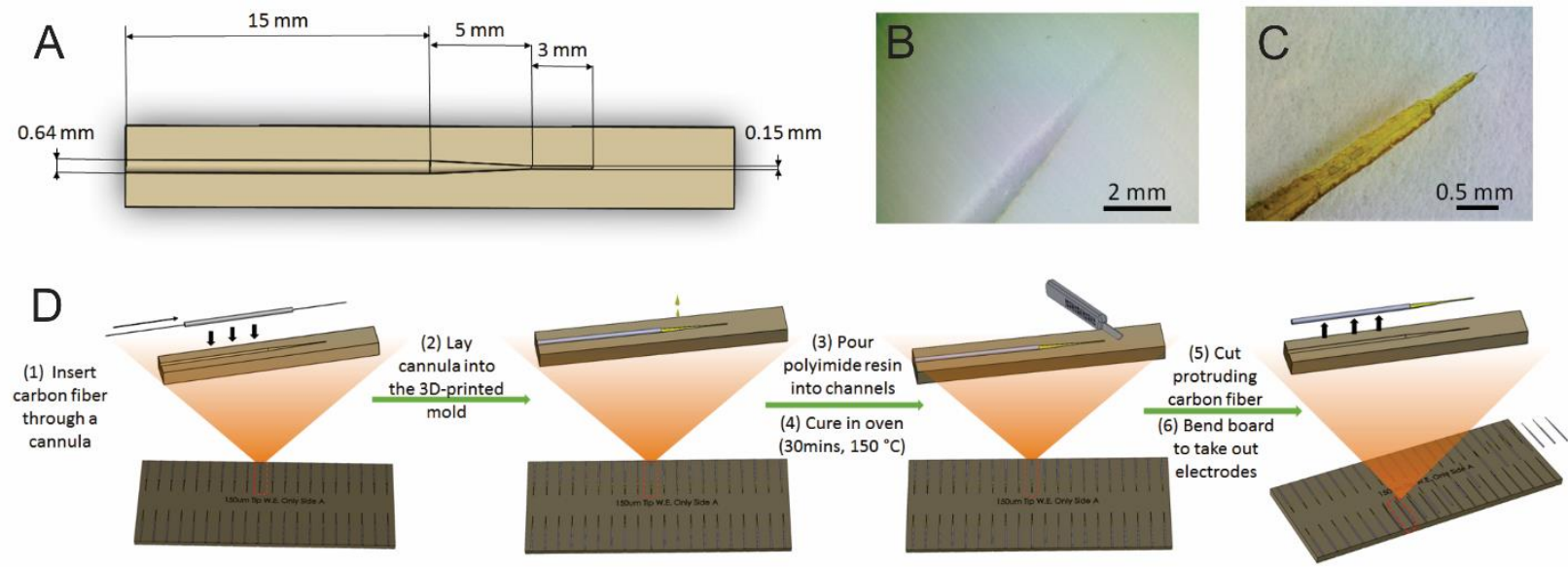

Figure 6.1: 3D-printed mold design and polyimide-insulated carbon fiber microelectrodes fabrication process. (A) Close-up of the design of one channel for 3D-printed microelectrode fabrication (B) image of the tip from a 3D-printed mold, scale bar: $2 \mathrm{~mm},(\mathrm{C})$ image of a polyimideinsulated carbon fiber microelectrode fabricated using the mold shown in B, scale bar: $0.5 \mathrm{~mm}$, (D) scheme of the process of microelectrode fabrication. 
The process of making the polyimide-insulated CFME is shown in figure 6.1D. An individual carbon fiber was inserted through a 23 gauge cannula needle, which was laid into the wide part of the mold. Polyimide resin was then poured into the mold channels, filling the tapered and tip part and sealing it to the cannula. It takes about 20 minutes to fill 40 channels with polyimide resin, and the resin was cured for 30 minutes in the oven at $150^{\circ} \mathrm{C}$. An additional layer of polyimide resin can be applied if required and cured for another 30 minutes at $150{ }^{\circ} \mathrm{C}$. The protruding carbon fiber is trimmed in order to make a cylindrical working electrode, typically around 100-150 $\mu \mathrm{m}$ long.

The 3D-printed mold is primarily made of acrylonitrile butadiene styrene (ABS), which provides a non-stick surface for the polyimide resin. The use of this material allowed the removal of the polyimide-insulated CFMEs by simply bending the flexible polymer mold and removing the electrode with tweezers. The 3D-printed molds were reused four times in this study before the detail of the device began to deteriorate. The average cost of materials for each electrode is less than 20 cents, with costs for a batch including only $\$ 1$ for the polyimide resin, less than $\$ 0.25$ for the carbon fiber, and $\$ 18$ for the 3D-printed mold of 40 channels that is used 4 times. The total time-cost for the 40 microelectrode fabrication is less than 2 hours, making for an economical fabrication procedure that can be developed as cheap alternative to the commercially available neurochemical microsensors in the market. For true batch fabrication in the future, filling of the mold could be automated by a robot and the length of the fiber protruding controlled upon laying it in the channel.

The 3D printing technology allowed consistency in electrode production as well as flexibility to change designs if needed. Mold designs can easily be shared as CAD files and then either modified or printed in another location. Thus, this method enables customization in design of the microelectrode system based on the different application requirements. Moreover, our 3D mold fabrication process could be used with other insulating agents such as epoxy ${ }^{5}$ or paraffin. ${ }^{6}$ This fabrication method is not limited to carbon fibers but could be used with many other electrode 
materials, such as carbon nanotube yarns, or metal wires. ${ }^{22,23} 3 \mathrm{D}$ printing is an emerging technology that is continuously progressing. ${ }^{13}$ The resolution of the molds varies by printer specifications, and we could make channels as small as $50 \mu \mathrm{m}$ diameter, but the printing was less consistent and some molds were not well tapered. Thus, we chose channels with $150 \mu \mathrm{m}$ diameter tips because our printer could reliably and consistently produce these. However, as the method advances, resolution is improved, and high resolution 3D printers becoming more accessible, the fabrication of smaller electrodes with smaller tip diameters will be possible.

\subsubsection{Electrochemical Characterization in Vitro and in Vivo}

Polyimide-insulated CFMEs were electrochemically characterized for the detection of dopamine using fast-scan cyclic voltammetry (FSCV). Dopamine is an important neurotransmitter in mammalian physiology that regulates locomotion and reward. ${ }^{24}$ In Figure 6.2, electrochemical properties of polyimide-insulated electrodes and traditional glass CFMEs are compared. The background-subtracted cyclic voltammograms (CV) of $1 \mu \mathrm{M}$ dopamine are similar because the length of the protruding fiber was the same, $150 \mu \mathrm{m}$. The oxidation peak for dopamine is not statistically different for glass-insulated CFMEs $(66 \pm 3 n A, n=4)$ and polyimide-insulated CFMEs (64 $\pm 2 \mathrm{nA} ; \mathrm{n}=4$; t-test, $\mathrm{p}=0.64$ ); thus, exposure to the polymer 3D printed mold or presence of the insulating polyimide resin does not disturb the reaction of dopamine at the carbon surface. However, the polyimide-insulated CFMEs have smaller background currents (Figure 6.2B, 1100 $\pm 100 \mathrm{nA} ; \mathrm{n}=4)$ than glass-insulated CFMEs (1570 $\pm 50 \mathrm{nA} ; \mathrm{n}=4$; t-test, $\mathrm{p}=0.0063)$. The larger background current of glass-insulated electrodes is due to the additional capacitance of the glass, which has a dielectric constant of 6 compared to the dielectric of polyimide, which is $3 .{ }^{25}$ The thickness of the insulations are also different, with thinner glass than the polyimide, which could allow ions to conduct through the glass. Despite the differences in background charging currents, the dopamine oxidation current of the electrode is not dependent on the type of insulation, as expected. 

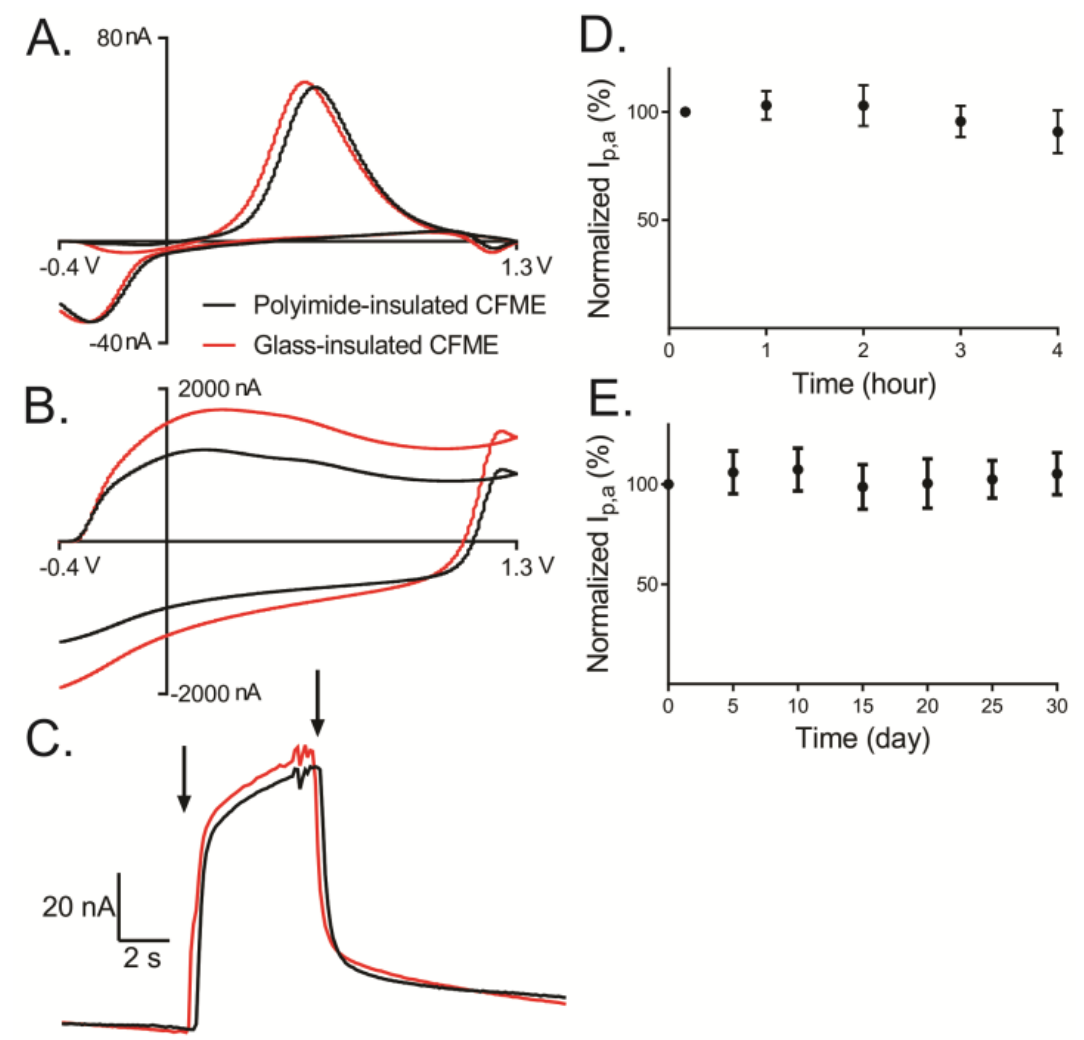

Figure 6.2: Electrochemical characterization of polyimide-insulated CFMEs. (A) Example background subtracted cyclic voltammogram (CV) of $1 \mu \mathrm{M}$ dopamine at a polyimide-insulated CFME (black) and glass-insulated CFME (red). (B) Background charging currents for the same electrodes in PBS buffer. (C) Current vs. time plot for the same electrodes during a flow injection analysis experiment; the first arrow indicates the injection of dopamine bolus, and the second arrow shows when flow is switched back to PBS buffer. (D) 4-hour stability test of response to 1 $\mu \mathrm{M}$ dopamine at polyimide-insulated CFMEs $(n=6)$. Waveform was continually applied and measurements taken every hour. (E) 30-day long term stability test at polyimide-insulated CFMEs $(n=4)$, Measurements were taken every 5 days. Data obtained in $(D)$ and $(E)$ were normalized to initial current values for peak oxidation of dopamine.

Figure $6.2 \mathrm{C}$ shows the temporal response of the CFMEs to a bolus of dopamine. The temporal response is not dependent on the type of insulation, although the response is not perfectly square due to adsorption/desorption kinetics. ${ }^{26}$ The consistent time response proves the polyimide-insulated microelectrodes are well insulated and that the polyimide is not rough at the end, trapping dopamine near the electrode surface.

To evaluate and confirm the electrode stability, the waveform was continuously applied to the electrode while the electrode was immersed in a buffer solution and the response to dopamine 
characterized over a 4 hour period, a typical time length of an in vivo stimulated release experiment. ${ }^{20}$ Figure $6.2 \mathrm{D}$ shows the dopamine oxidation signal is constant for 4 hours of continuous scanning and that the surface is stable over the time length of a biological experiment. Shelf life stability was examined by testing electrodes over a month after manufacturing. No change in oxidation current or temporal resolution was observed throughout the one month period (Fig. 6.2E). The polyimide-insulated CFMEs are stable for several hours of experimental use and have a good shelf life, allowing them to be made in batches and stored until needed.

To determine the applicability of the polyimide-insulated CFMEs as in vivo sensors, stimulated dopamine release was measured in anesthetized rats. Stimulation pulse trains were applied (300 $\mu \mathrm{A}, 30-120$ pulses, $60 \mathrm{~Hz}$ ) to the dopamine cell bodies, and the dopamine response was recorded by polyimide-insulated CFME in the caudate putamen. Figure 6.3A shows dopamine concentrations recorded at different stimulated pulses by polyimide-insulated CFMEs ( $n=4$ rats). Data was converted from peak currents to concentration based on postcalibration factors. As observed in the figure, a higher number of pulses elicit larger release of dopamine on the microelectrode surface, and the electrode is sensitive enough to detect dopamine release as low as $170 \mathrm{nM}$. Figures 6.3 B and C show example CV and current versus time plots of dopamine detection at a polyimide-insulated CFME in vivo. 

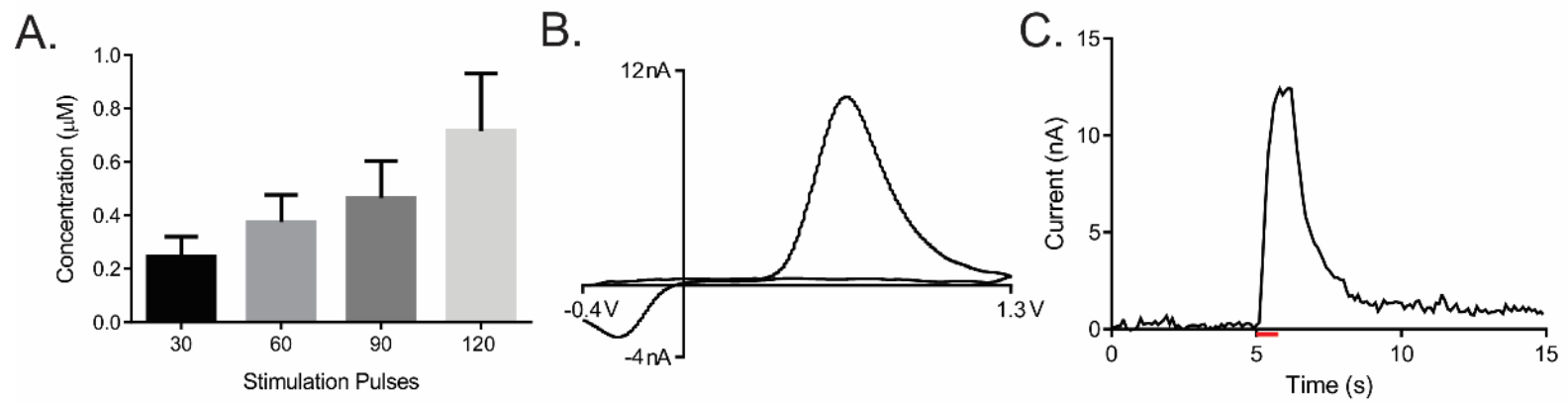

Figure 6.3: Dopamine detection in vivo at polyimide-insulated CFMEs. (A) Peak cyclic currents were recorded at different stimulated pulses. Data were converted to concentration on the basis of post calibration factors ( $n=4$ rats). (B) Sample CV of stimulated dopamine release detected from microelectrodes placed in the caudate putamen with stimulation pulse trains of 60 pulses at $60 \mathrm{~Hz}$. (C) Associated current vs time plot. Red bar indicates the stimulation duration.

Polyimide-insulated CFMEs could potentially be used in higher order mammals or humans in the future, given their biocompatibility and shatter resistance. Measurement of dopamine release has been demonstrated previously in the caudate of a human patient during electrode implantation surgery. ${ }^{7}$ The CFMEs used were insulated with polyimide-coated, fused silica capillaries, which are similar in material to the polyimide resin used in this work. The promising properties of this sensor, as well as the ability to batch fabricate reproducibly, could lead to promising new electrode fabrication methods in the future. 


\subsection{Conclusions}

In summary, we have devised a novel method of mass-fabricating CFMEs for the electrochemical detection of neurotransmitters such as dopamine. The polyimide-insulated electrodes are biocompatible and flexible and can be implanted in vivo. 3D printing provides an easy way to reproducibly fabricate molds, and electrodes can be customized according to experimental needs. As printing technology continues to evolve, new applications will be enabled, including smaller probes. The polyimide-insulated CFMEs have comparable oxidation currents, stability, and temporal resolution to the conventionally used glass-insulated CFMEs. The electrodes were successfully used as in vivo sensors for sensitive detection of stimulated dopamine in caudate putamen. With advantages such as low-cost, high bio-compatibility, easy batch fabrication, and customizable design, this novel microelectrode fabrication method will be helpful for low cost, batch fabrication of carbon and metal material-based microelectrodes. 
Yang 171

\subsection{References}

1) M.L. Huffman and B.J. Venton, Carbon-fiber microelectrodes for in vivo applications, Analyst, 2009, 134, 18-24.

2) R.B. Keithley, P. Takmakov, E.S. Bucher, A.M. Belle, A. Owesson-White, J. Parl and R.M. Wightman, Higher sensitivity dopamine measurements with faster-scan cyclic voltammetry, Anal. Chem., 2011, 83, 3563-3571.

3) A.G. Ewing, M.A. Dayton and R.M. Wightman, Pulse voltammetry with microvoltammetric electrodes, Anal. Chem., 1981, 53, 1842-1847.

4) P.S. Cahill, Q.D. Walker, J.M. Finnegan,, G.E. Mickelson, E.R. Travis and R.M. Wightman, Microelectrodes for the measurement of catecholamines in biological systems, Anal. Chem., 1996, 68, 3180-3186.

5) A.G. Zestos, M.D. Nguyen, B.L. Poe, C.B Jacobs and B.J. Venton, Epoxy insulated carbon fiber and carbon nanotube fiber microelectrodes, Sensors and Actuators B: Chem., 2013, 182, 652-658.

6) E.S. Ramsson, D. Cholger, A. Dionise, A. Andrus, N. Poirier and R. Curtiss, Characterization of Fast-Scan Cyclic Voltammetric Electrodes Using Paraffin as an Effective Sealant with In Vitro and In Vivo Applications, PLoS One, 2015, 10, e0141340.

7) K.T. Kishida, S.G. Sandberg, T. Lohrenz, Y.G. Comair, I. Saez, P.E.M. Phillips and P.R. Montague, Sub-second dopamine detection in human striatum, PLoS One, 2011, 6, e23291.

8) A.J. Garwon, R.S. Martin and S.M. Lunte, Fabrication and evaluation of a carbon-based dualelectrode detector for poly (dimethylsiloxane) electrophoresis chips, Electrophoresis, 2001, 22, 242-248.

9) L.V. Sokolov, A.A. Zhukov, N.M. Parfenov and S.O. Igoshin, Study of the technological limitations of photolithography for the relief surface of a SOI wafer during formation of the threedimensional micromechanical structure of an integral tensoconverter, J. Surface Inv., 2013, 7, 178-180.

10) M.D. Symes, P.J. Kitson, J. Yan, C.J. Richmond, G.J.T. Cooper, R.W. Bowman, T. Vilbrandt and L. Cronin, Integrated 3D-printed reactionware for chemical synthesis and analysis, Nature Chemistry, 2012, 4, 349-354.

11) F. Rengier, A. Mehndiratta, H. von Tengg-Kobligk, C. M. Zechmann, R. Unterhinninghofen, H.U. Kauczor and F. L. Giesel, 3D printing based on imaging data: review of medical applications, J. CARS, 2010, 5, 335-341.

12) H.N. Chia and B.J. Wu, Fabrication and Applications of Micro/Nanostructured Devices for Tissue Engineering, J. Bio. Eng., 2015, 9, DOI: 10.1186/s13036-015-0001-4.

13) A. Ambrosi, J.G.S. Moo and M. Pumera, Helical 3D - Printed Metal Electrodes as Custom Shaped 3D Platform for Electrochemical Devices, Adv. Functional Materials, 2015, 26, 698-703.

14) H. Ragones, D. Schreiber, A. Inberg, O. Berkh, G. Kósa, A. Freeman and Y. ShachamDiamand, Disposable electrochemical sensor prepared using 3D printing for cell and tissue diagnostics, Sensors and Actuators B: Chem., 2015, 216, 434-442.

15) J.J. Clark, S.G. Sandberg, M.J. Wanat, J.O. Gan, E.A. Horne, A.S. Hart, C.A. Akers, J.G. Parker, I. Willuhn, V. Martinez, S.B. Evans, N. Stella and P.E. Phillips, Chronic microsensors for 
longitudinal, subsecond dopamine detection in behaving animals, Nat. Methods, 2010, 7, 126129.

16) P.J. Rousche, D.S. Pellinen, D.P. Pivin, J.C. Williams, R.J. Vetter and D.R. Kipke, Flexible polyimide-based intracortical electrode arrays with bioactive capability, IEEE Trans. Biom. Eng., 2001, 48, 361-370.

17) X. Navarro, T.B. Krueger, N. Lago, S. Micera, T. Stieglitz and P. Dario, A critical review of interfaces with the peripheral nervous system for the control of neuroprostheses and hybrid bionic systems, J. Periph. Nerv. Syst., 2005, 10, 229-258.

18) N. Lago, K. Yoshida, K.P. Koch and X. Navarro, Assessment of biocompatibility of chronically implanted polyimide and platinum intrafascicular electrodes, IEEE Trans. Biom. Eng., 2007, 54, 281-290.

19) D.L. Robinson, B.J. Venton, M.L. Heien and M.R. Wightman, Detecting subsecond dopamine release with fast-scan cyclic voltammetry in vivo, Clin. Chem., 2003, 10, 1763-1773.

20) M.K. Zachek, P. Takmakov, J. Park, M.R. Wightman and G.S. McCarty, Simultaneous monitoring of dopamine concentration at spatially different brain locations in vivo, Biosens. Bioelectron., 2010, 25, 1179-1185.

21) M.L. Heien, A.S. Khan, J.L. Ariansen, J.F. Cheer, P.E. Phillips, K.M. Wassum and M.R. Wightman, Real-time measurement of dopamine fluctuations after cocaine in the brain of behaving rats, PNAS, 2005, 102, 10023-10028.

22) A.E. Lindsay and D. O'Hare, A comparative study of thin film insulation techniques for gold electrodes, Electrochimica Acta, 2006, 51, 6572-6579.

23) P. Sun, Z. Zhang, J. Guo and Y. Shao, Fabrication of nanometer-sized electrodes and tips for scanning electrochemical microscopy, Anal. Chem., 2001, 73, 5346-5351.

24) J.H. Baik, Front. Dopamine signaling in reward-related behaviors, Neural Circuits, 2013, 7, 152.

25) G. Maier, Low dielectric constant polymers for microelectronics, Prog. Pol. Sci., 2001, 26, 365.

26) B.D. Bath, H.B. Martin, R.M. Wightman and M.R. Anderson, Dopamine adsorption at surface modified carbon-fiber electrodes, Langmuir, 2001, 17, 7032-7039. 
Chapter 7: High Performance, Low Cost Carbon Nanotube Yarn based 3D Printed Electrodes Compatible with a Conventional Screen Printed Electrode System 


\section{Abstract}

3D printing technology has been widely used as a rapid prototyping fabrication tool in several fields including electrochemistry. In this work, we incorporate 3D printing technology with carbon nanotube yarns for electrochemical sensing of dopamine in the presence of ascorbic acid and uric acid. The novel 3D printed electrode provides a circular concavity detection zone with grooves to insert three electrodes. The electrodes connections are fully compatible with conventionally used screen printed electrode workstation setups. The CNT yarn 3D printed electrode showed excellent electrocatalytic activity for the redox reaction of dopamine (DA) in the presence of ascorbic acid (AA) and uric acid (UA). Three well-defined sharp and fully resolved anodic peaks were found with the peak potentials using CV at about $50 \mathrm{mV}, 305 \mathrm{mV}$, and 545 $\mathrm{mV}$ for $\mathrm{AA}, \mathrm{DA}$, and $\mathrm{UA}$ respectively and using $\mathrm{DPV}$ at $91 \mathrm{mV}, 389 \mathrm{mV}$, and $569 \mathrm{mV}$, respectively. DA detection limit was $0.87 \pm 0.09 \mu \mathrm{M}$. And the CNT yarn 3D printed electrode displayed high reproducibility and stability. The electrode design also enables the study of electrode reactions at the sidewall of CNTs, which cannot be performed using electrodes made by conventional fabrication methods. The new fabrication method with low-cost, customizable design, and compatibility with existing screen printed electrodes workstations provides a new platform to prototype new electrode materials for electrochemistry. 


\subsection{Introduction}

The recent development of 3D printing based rapid prototyping leads to a consumeroriented availability of desktop or bench-top printing devices. ${ }^{1}$ The 3D printing process allows 3D objects to be fabricated in a bottom-up, additive fashion directly from digital designs, with no milling or molding. ${ }^{2}$ This progress, often described as a revolution in the manufacturing industry, enables the cost-effective use of self-developed and individually designed labware and devices in the bio-science field. ${ }^{3,4} 3 \mathrm{D}$ printing approaches are increasingly used in several scientific and medical applications, such as tissue-growth scaffolds, ${ }^{5,6}$ microvascular systems, ${ }^{7}$ orthopedic implants, ${ }^{8}$ rehabilitation aid tools, ${ }^{9}$ electronic device, ${ }^{10}$ and chemical reactors. ${ }^{11}$ Electrochemistry is another branch of science that can benefit from 3D-printing technologies, paving the way for both design and fabrication of cheaper, higher performing, and customizable electrochemical devices. ${ }^{12,13}$ To address the needs of on-site analysis, 3D printing can help moving away from the commonly used electrodes and cells for various materials. Recently, we reported a 3D printed mold assisted novel carbon fiber microelectrodes (CFMEs) batch fabrication method using polyimide resin as sealing agent for the in vivo detection of dopamine. 3D printing provides an easy way to reproducibly fabricate molds, and the produced electrodes can be customized according to experimental needs with various materials. ${ }^{14}$

Carbon nanomaterials, such as graphene or carbon nanotubes (CNTs), are commonly used for electrochemical detection of biomolecules because of their promising electron transfer kinetics, high conductivity, good antifouling property and biocompatibility. ${ }^{15}$ Screen printed electrode (SPE) fabrication method is one of the most efficient approach to apply carbon nanomaterials for electrochemical applications. ${ }^{16-20}$ However, an ink needs to be made containing certain surfactants to improve carbon nanomaterial dispersion and mineral binders or insulating polymers to improve the adhesion onto the substrate. The exact ink formulation and composition are usually patented by companies and are not disclosed to the users. Moreover, SPE fabrication 
method is not suitable for macrostructured materials, such as fibers. Therefore, the application of novel carbon nanomaterials using SPE fabrication method has limited choices of materials. The incorporation of $3 \mathrm{D}$ printing and electrochemical sensing applications would not only provide customizable design and reduce the cost, but also provide an approach to fabricate electrodes with materials that are not easily fabricated into sensors. In this work, we designed a novel 3D printed electrode fabrication method to incorporate fibers into electrodes that are compatible for the commercially available SPE workstation setups.

CNT yarns are a macrostructure of CNTs fabricated by solid state processes, and no dispersion is required. ${ }^{21,22}$ Compared to graphene and CNTs paste, CNT yarns have higher purity. ${ }^{23}$ Moreover, in comparison to randomly distributed and tangled CNTs produced by dip coating or screen printing, well-aligned CNT yarns have the advantages of high electroactivity, chemical stability, high conductivity, controllable size, and promising anti-fouling properties. ${ }^{15,24,25}$ The CNT yarn has intrinsically abundant adsorption sites for neurotransmitters, such as dopamine, and has already been used for sensitive electrochemical sensing. ${ }^{26,27}$ Moreover, most sensor designs are based on maximizing exposure of edge plane defects which are on the end of CNT yarn, ${ }^{28}$ while whether CNT sidewalls are electrocatalytic for dopamine are still not well understood. ${ }^{28-31}$ Thus, there is a dichotomy between the practical and the fundamental science, and the 3D printed electrode designed for CNT yarn as a new method to assemble CNTs based electrochemical sensors elucidates the extent to which electron transfer occurs at either edge plane sites or basal plane sites. The electrode fabrication method using 3D printing would provide a low-cost devices specially designed to work with micro-volumes of sample, and a novel approach for the applications and fundamental electrochemistry study on various fiber materials. 


\subsection{Methods}

\subsubsection{Reagents and Materials}

Dopamine hydrochloride, uric acid, and ascorbic acid were purchased from Sigma-Aldrich (St. Louis, MO). Dopamine stock solutions with concentration of $10 \mathrm{mM}$, uric acid and ascorbic acid stock solutions with concentration of $100 \mathrm{mM}$ were prepared in $\mathrm{HClO}_{4}$, and were diluted daily to the desired concentration in phosphate buffered saline $(131.3 \mathrm{mM} \mathrm{NaCl}, 3.00 \mathrm{mM} \mathrm{KCl}, 10 \mathrm{mM}$ $\mathrm{NaH}_{2} \mathrm{PO}_{4}, 1.2 \mathrm{mM} \mathrm{MgCl}_{2}, 2.0 \mathrm{mM} \mathrm{Na}_{2} \mathrm{SO}_{4}$, and $1.2 \mathrm{mM} \mathrm{CaCl}_{2}$ with the $\mathrm{pH}$ adjusted to 7.4).

\subsubsection{Electrode Fabrication}

Molds were designed in Autodesk Inventor Professional 2014 Student Edition, converted to an .STL file, and subsequently printed by the Department of Mechanical Engineering at University of Virginia. The 3D printed polymer molds were manufactured on a Stratasys Connex 500 Model 1 Poly-Jet 3D printer (Stratasys Ltd, MN), which has 8 print heads with 96 nozzles per head. Water jets were used to remove the support. Rigid opaque black material (VeroBlackPlus RGD875, mainly acrylonitrile butadiene styrene, Stratasys) was used because of its suitability for rapid tooling with dimensional stability and fine detail. The heads heat up to $60^{\circ} \mathrm{C}$, and the $\mathrm{Z}$ axis resolution with this material was $30 \mu \mathrm{m}$. A piece of commercially available carbon nanotube yarn (CNT yarn, 10-25 $\mu \mathrm{m}$ in diameter, 1-2 cm long, General Nano, LLC, Cincinnati, OH) was used as working electrode. The homemade reference electrode was a silver/silver chloride electrode with diameter of $250 \mu \mathrm{m}$, fabricated by applying $2 \mathrm{~V}$ potential to a $4 \mathrm{~cm}$ long silver wire for 30 seconds in concentrated hydrochloric acid. The counter electrode is a $\sim 2 \mathrm{~cm}$ long silver wire (ESPI Metals, Ashland, OR) with a diameter of $250 \mu \mathrm{m}$. Electrodes materials were sealed with $5 \mathrm{~min}$ epoxy Loctite (Henkel Corporation, Westlake $\mathrm{OH}$ ) which was allowed to fully cure for $24 \mathrm{~h}$. The connection between electrode material and SPE adapter is achieved by stainless steel plates, 
and silver/silver chloride paste (The Gwent Group, United Kingdom) is used to ensure the conductivity between the plates and electrode materials.

\subsubsection{Apparatus}

All electrochemical measurements were performed on a Gamry electrochemical workstation (Gamry Reference 600, Gamry Instruments, USA). A commercial universal screen printed electrode cable connector (Metrohm USA Inc, FL) is used to connect the electrochemical workstation and the 3D printed electrodes. Scanning electron microscope (SEM) images were taken on Merlin field emission SEM (Zeiss, Thornwood, NY) with a secondary electron detector using an accelerating voltage of $2 \mathrm{kV}$ and a working distance of $5.0 \mathrm{~mm}$. 


\subsection{Results and Discussion}

\subsubsection{CNT Yarn based 3D Printed Electrode Fabrication}

Figure 7.1A shows the design of a 3D printed electrode, with the dimensions of $30 \mathrm{~mm} \times$ $10 \mathrm{~mm} \times 2 \mathrm{~mm}$. There are three grooves to place the working, reference and counter electrodes in the 3D printed electrode substrate. The detection zone is acircular concavity that can hold a drop of solution across the three electrodes. Three metal plates are used to connect the electrodes to a potentiostat via a SPE adapter cable. As shown in Figure 7.1B, three grooves are for silver counter electrode (500 $\mu \mathrm{m}$ in diameter), CNT yarn working electrode ( $250 \mu \mathrm{m}$ in diameter, bigger than the electrode), and $\mathrm{Ag} / \mathrm{AgCl}$ reference electrodes (250 $\mu \mathrm{m}$ in diameter) from bottom to top, respectively. Epoxy resin is applied as sealing agent to fix electrode materials in the grooves and limit the area of detection zone after placing all the three electrodes in grooves. Moreover, the intrinsically hydrophobic property of epoxy sealing would also help restrict the sample solution $(\sim 50 \mu \mathrm{L})$ in the $4 \mathrm{~mm}$ diameter $(0.6 \mathrm{~mm}$ deep) circular concavity detection area. The distance between working and reference electrodes $(300 \mu \mathrm{m})$ is half of the distance between working and counter electrodes $(600 \mu \mathrm{m})$, to reduce the ohmic drop. The connections between three electrodes to SPE adapters are achieved by stainless steel plates with width of $2 \mathrm{~mm}$ placed in the grooves with the spacing between each plates of $0.8 \mathrm{~mm}$ (Figure 7.1B). Electrodes can be easily revised to fit the adapters for different brands of widely used and commercially available SPEs from Pine Instrumentation Inc., DropSens, or $\mathrm{CH}$ Instruments. 

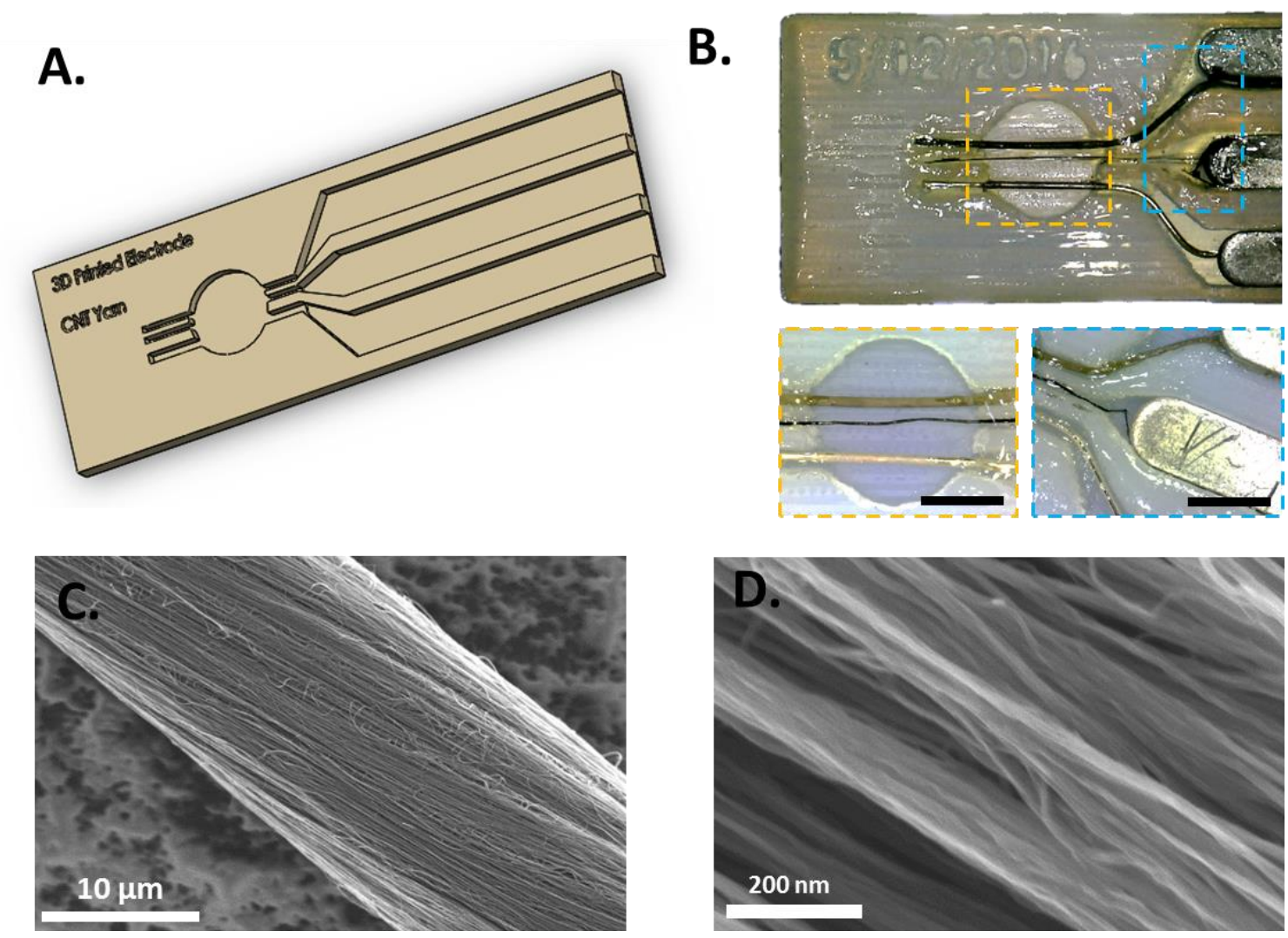

Figure 7.1: Illustration of electrode design and CNT yarn 3D Printed Electrode. (A) Close-up of the design of the 3D printed electrode substrate for CNT yarn. (B) Image of the CNT yarn 3D printed electrode top-view. Circles shows zoom-in images of the detection area and electrode material-metal plate connections. Scale bar: $2 \mathrm{~mm}$. (C) SEM Image of a CNT yarn with spun well aligned CNT bundles and (D) zoomed in SEM image on the same CNT yarn sample.

The 3D-printed electrode substrate is primarily made of acrylonitrile butadiene styrene (ABS), which is not soluble in aqueous solutions. With the help of water jets, $30 \mu \mathrm{m}$ resolution is achieved by using ABS, which is suitable for our design with channels of hundreds of microns wide. The average cost of materials including ABS, epoxy, and electrode materials is less than 1 dollar per electrode. More importantly, the 3D printed electrode design allows any materials that are fiber-like to be made into an electrode e.g. carbon fiber, CNT yarn/fiber, metal or polymer fibers. These materials are infeasible or extremely difficult to achieve by using traditional SPE, helping extensively broaden the electrochemical applications. 
Yang | 181

\subsubsection{Electrochemical Characterizations}

In this work, CNT yarn is used as working electrode material. CNT yarn is a macrostructure of CNTs with well-aligned multiwall CNTs (MWCNTs) bundles spun through the yarn, as shown in Figure 7.1C. The continuous MWCNTs with diameter about $30-50 \mathrm{~nm}$ (Figure 7.1D) have abundant $\mathrm{sp}^{2}$ hybridized carbons compared to $\mathrm{sp}^{3}$ hybridized carbons which are mainly located at the ends of CNT bundles. The 3D printed electrode using CNT yarn as working electrode was electrochemically characterized using cyclic voltammetry scanning from -0.2 to $0.6 \mathrm{~V}$ and back at $200 \mathrm{mV} / \mathrm{s}$. The response to $300 \mu \mathrm{M}$ dopamine (DA, red curve) or a mixture of $300 \mu \mathrm{M}$ dopamine (DA), $1 \mathrm{mM}$ ascorbic acid (AA), and $1 \mathrm{mM}$ uric acid (UA) (black curve) is shown in Fig. 2A For DA (Fig. 2A(a)), the cathodic and anodic peaks appear at about $280 \mathrm{mV}$ and $330 \mathrm{mV}$, respectively, a substantial positive shift of the oxidation peak potential compared to other carbon nanomaterials. ${ }^{16,32}$ The separation between the oxidation and reduction peak potential $\left(\Delta \mathrm{E}_{p}\right)$ is 53 $\pm 2 \mathrm{mV}$ from four electrodes, which is smaller than several previous works using CNTs based SPEs, ${ }^{16,32}$ indicating faster electron transfer kinetics. AA and UA are common interferences in tissue, ${ }^{15}$ and the DA selectivity over AA and UA were tested at CNT yarn 3D printed electrodes. Figure 7.2A(b) shows the $\mathrm{CV}$ of the mixture at the CNT yarn 3D printed electrode has three welldefined sharp, and fully resolved anodic peaks at $50 \mathrm{mV}, 305 \mathrm{mV}$ and $545 \mathrm{mV}$, corresponding to the oxidation of AA, DA, and UA, respectively. The calculated oxidation peak potential separations are $255 \mathrm{mV}$ for AA-DA, $240 \mathrm{mV}$ for DA-UA, and $495 \mathrm{mV}$ for UA-AA, demonstrating promising selectivity for simultaneous determination of these three species. Figure 7.2B displays differential pulse voltammogram (DPV) obtained at the CNT yarn 3D printed electrode for a mixture containing $300 \mu \mathrm{M} \mathrm{DA}, 1 \mathrm{mM} \mathrm{AA}$, and $1 \mathrm{mM} \mathrm{UA}$. The potentials for AA, DA, and UA are at $91 \mathrm{mV}$, $389 \mathrm{mV}$, and $569 \mathrm{mV}$, respectively, and the separations for AA-DA, DA-UA, and AA-UA are 298 $\mathrm{mV}, 180 \mathrm{mV}$, and $478 \mathrm{mV}$, respectively. Thus, the CNT yarn 3D printed electrode can distinguish DA, UA, and AA using both CV and DPV. 

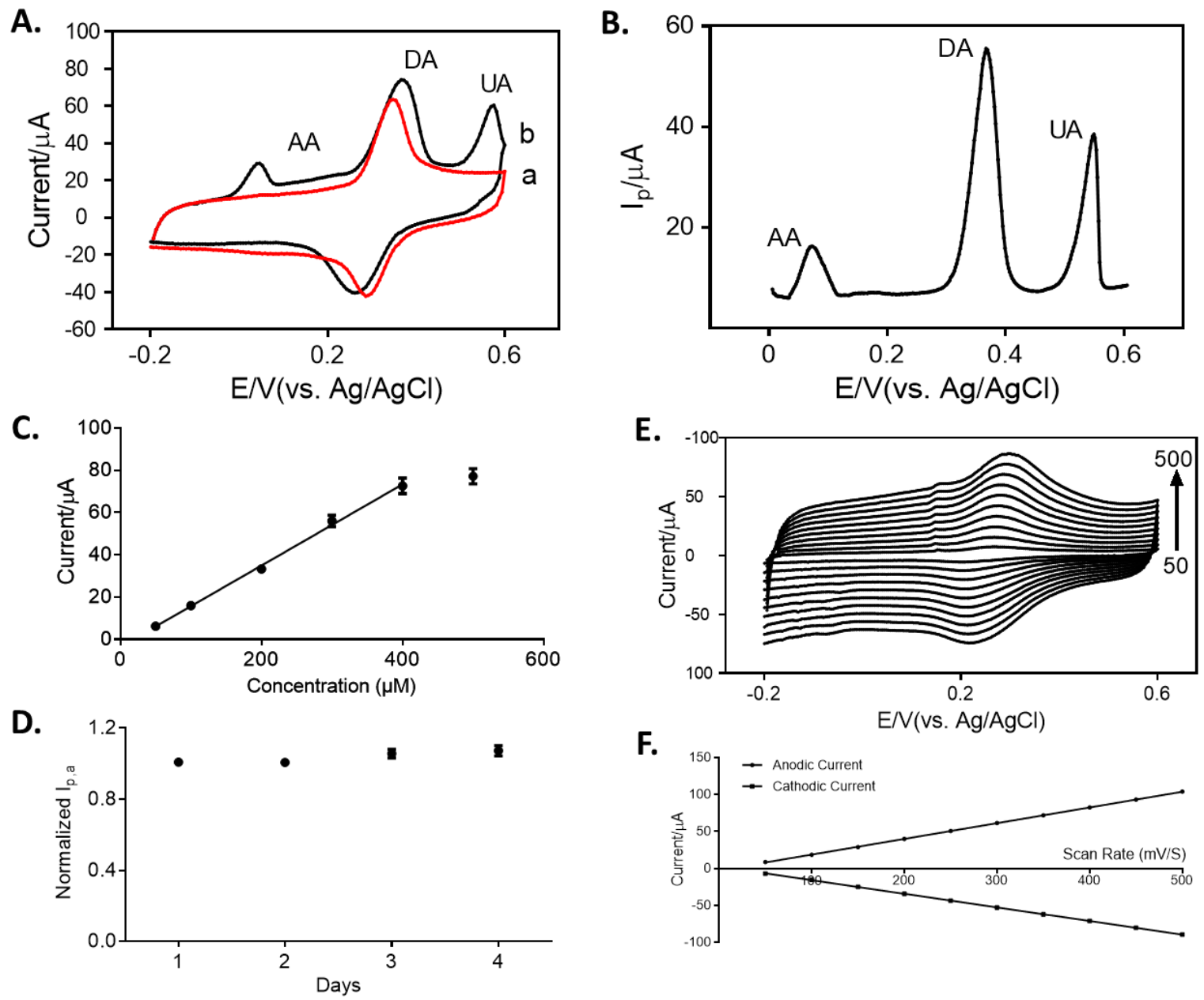

Figure 7.2: Electrochemical characterization of CNT yarn 3D printed electrodes. (A) Cyclic voltammogram of $300 \mu \mathrm{M}$ dopamine PBS (pH 7.4) solution (curve a) and a mixture of $300 \mu \mathrm{M}$ dopamine, $1 \mathrm{mM}$ uric acid and $1 \mathrm{mM}$ ascorbic acid solution (curve b). Scan rate $200 \mathrm{mV} / \mathrm{s}$. (B) DPV of $300 \mu \mathrm{M}$ dopamine in the presence of $1 \mathrm{mM}$ uric acid and $1 \mathrm{mM}$ ascorbic acid. (C) Anodic current to different concentrations of dopamine in a range of 50 to $500 \mu \mathrm{M}(\mathrm{n}=4)$, with scan rate of $200 \mathrm{mV} / \mathrm{s}$ from -0.2 to $0.6 \mathrm{~V}$. A linear range from 50 to $400 \mu \mathrm{M}$ has $R^{2}$ of 0.9817 . (D) The stability experiment was performed by testing the response of CNT yarn 3D printed electrodes $(n=4)$ to $300 \mu \mathrm{M}$ dopamine every day for four days, with scan rate of $200 \mathrm{mV} / \mathrm{s}$ from -0.2 to $0.6 \mathrm{~V}$. (E) Cyclic voltammogram of $50 \mu \mathrm{M}$ dopamine at different scan rates from 50 to $500 \mathrm{mV} / \mathrm{s}$, and $(F)$ the relations between the anodic/cathodic peak current and scan rate.

CNT yarn 3D printed electrodes were used to detection different concentrations from 50 $\mu \mathrm{M}$ to $500 \mu \mathrm{M}$ DA using $\mathrm{CV}$ and the plot of oxidation current versus concentration is shown in Figure 7.2C $(n=4)$. A linear response was obtained from $50 \mu M$ to $400 \mu M\left(R^{2}=0.9817\right)$, and the limit of detection (LOD) is $0.87 \pm 0.09 \mu \mathrm{M}(\mathrm{S} / \mathrm{N}=3)$. To assess the stability of the electrodes, 
the dopamine CV peak current was measured once per day for 4 days (Figure 7.2D). The calculated RSD is only $0.986 \%$, indicating promising shelf stability as well as its low susceptibility to electrode fouling caused by oxidized products that could strongly absorb onto the electrode surface. ${ }^{33,34}$ The electrode-to-electrode reproducibility was also checked by comparing four CNT yarn 3D printed electrodes prepared under the same conditions. The RSD for dopamine was $4.12 \%$, confirming that the fabrication method is highly reproducible.

Since most of the CNT yarn studies have only investigated the electrochemical performance at its polished tip, the electroactivity of the sidewall of CNT yarn is not well understood..$^{27,35}$ For example, the redox reaction of dopamine depends on the surface oxygencontaining functional groups, surface roughness, and $\pi-\pi$ stacking reversibility. ${ }^{15,36-40}$ In contrast to most sensor designs, which are based on maximizing exposure of edge plane defects which are on the end of CNT yarn ${ }^{28}$, the novel 3D printing assisted fabrication method provides a platform for the electrochemistry study of the reactivity of CNT sidewalls. The kinetics of electrode reaction were investigated by evaluating the effect of CV scan rate on the DA oxidation and reduction peak currents (Fig. 2E). Both the anodic and cathodic peak currents were proportional to scan rate in the range of 50 to $500 \mathrm{mV} / \mathrm{s}$ (Figure $7.2 \mathrm{~F}$ ). The linear regression equation for the anodic peak current was $I_{p, a}(\mu A)=2.33+0.21^{*} v(m V / s)$, with a correlation coefficient of $R^{2}=$ 0.9999 , and for the cathodic peak current was $I_{p, c}=-2.74-0.18^{*} v(m V / s)$, with $R^{2}=0.9999$. Both DA oxidation and reduction reactions at CNT yarn 3D printed electrodes are adsorption controlled processes, similar to DA redox reaction at the tips of CNT yarn and carbon fiber microelectrodes. ${ }^{27,34,35}$ The adsorption mechanism of DA reaction on CNTs and carbon fiber is due to the electrostatic interaction with the negatively charged oxygen-containing functional groups under physiological $\mathrm{pH} .{ }^{25,41,42}$ But due to the lack of $\mathrm{sp}^{3}$ hybridized carbons on the pristine CNT yarn sidewall, the $\pi-\pi$ stacking between dopamine and CNTs sidewall is likely to play an important role for the adsorption controlled process. 
A secondary oxidation peak at $160 \mathrm{mV}$ was observed for dopamine with $\mathrm{CV}$, especially at slower scan rate (Figure $7.2 \mathrm{E}$ ). The secondary oxidation is likely due to the oxidation of leucodopaminechrome (LDAC) to dopaminechrome (DAC), in the synthetic pathway of DA $\rightleftharpoons$ $\mathrm{DOQ} \rightarrow \mathrm{LDAC} \rightleftharpoons \mathrm{DAC}$. Similar results have been observed at a long-length (hundred micrometers) CNT electrodes, ${ }^{43}$ but not at other carbon electrodes such as carbon paste, graphene, fullerene, nanofiber, and graphite. The 3D printed electrode method provides a much easier fabrication approach than dispersing and coating long-length CNTs on a substrate made and therefore is a good platform to perform further fundamental electrochemical studies of CNT sidewall reactivity. Overall, the 3D printed electrode fabrication method provides a platform for testing the electrochemical reactivity of any fiber or wire electrode material with ease. The design has revealed interesting good electrocatalytic properties of CNT yarn sidewalls and enhancement of side reactions from cyclization products that are stabilized at the CNTs. 


\subsection{Conclusions}

In this work, we investigate a novel 3D printed platform for electrode fabrication that expands the electrochemical application of various fiber materials. The new fabrication method has the advantages of low-cost, customizable design, and high reproducibility. Moreover, the compatibility to existing SPE electrochemistry workstations minimizes the cost because no extra device/instrument is required. CNT yarn was used as electrode material, and with the DA LOD with $\mathrm{CV}$ is less than $1 \mu \mathrm{M}$. The CNT yarn electrode demonstrated promising selectivity of DA in the presence of AA and UA enabling simultaneous detection with CV or DPV. In addition, the good sensitivity and adsorption controlled process for DA demonstrates possible electroactivity of CNTs sidewalls, and indicates the 3D printed electrode method is a good platform for electrochemistry studies on sidewalls which are hard to study with conventional fabrication approaches. Future studies could examine the surface properties and electrochemical performance after pretreatments such as surface modifications or decorated with polymers, metals particle, or biomaterials because the CNT yarn sidewall is a highly homogenous structure

for sensing. ${ }^{44,45}$ The new 3D printed platform allows broad application of not only CNT yarn but also other materials such as metal or polymer fibers. 3D printed platforms could also potentially be applied as electrode arrays, which would allow the calibration and the analysis of several analytes simultaneously. 


\subsection{References}

(1) Lücking, T. H.; Sambale, F.; Beutel, S.; Scheper, T. 3D-Printed Individual Labware in Biosciences by Rapid Prototyping: A Proof of Principle. Eng. Life Sci. 2014, 51-56.

(2) Sandron, S.; Heery, B.; Gupta, V.; Collins, D. a; Nesterenko, E. P.; Nesterenko, P. N.; Talebi, M.; Beirne, S.; Thompson, F.; Wallace, G. G.; Brabazon, D.; Regan, F.; Paull, B. 3D Printed Metal Columns for Capillary Liquid Chromatography. Analyst 2014, 139 (24), 6343-6347.

(3) Yager, P.; Edwards, T.; Fu, E.; Helton, K.; Nelson, K.; Tam, M. R.; Weigl, B. H. Microfluidic Diagnostic Technologies for Global Public Health. 2006, 442 (July).

(4) Cook, T. R.; Dogutan, D. K.; Reece, S. Y.; Surendranath, Y.; Teets, T. S.; Nocera, D. G. Solar Energy Supply and Storage for the Legacy and Nonlegacy Worlds. Chem. Rev. 2010, 6474-6502.

(5) Lee, K.; Wang, S.; Dadsetan, M.; Yaszemski, M. J.; Lu, L. Enhanced Cell Ingrowth and Proliferation through Three-Dimensional Nanocomposite Scaffolds with Controlled Pore Structures. Biomacromolecules 2010, 11, 682-689.

(6) Mannoor, M. S.; Jiang, Z.; James, T.; Kong, Y. L.; Malatesta, K. A.; Soboyejo, W. O.; Verma, N.; Gracias, D. H.; Mcalpine, M. C. 3D Printed Bionic Ears. Nano Lett. 2013, 13, 2634-2639.

(7) Therriault, D.; White, S. R.; Lewis, J. A. Chaotic Mixing in Three-Dimensional Microvascular Networks Fabricated by Direct-Write Assembly. 2003, 2 (April), 265-272.

(8) Mackinnon, J.; Daurka, J. Use of 3D Printing in Orthopaedic Surgery. 2014, 2963 (May), 2963.

(9) Hsu, L. H.; Huang, G. F.; Lu, C. T.; Hong, D. Y.; Liu, S. H. The Development of a Rapid Prototyping Prosthetic Socket Coated with a Resin Layer for Transtibial Amputees. 2010, 34 (March), 37-45.

(10) Zhao, C.; Wang, C.; lii, R. G.; Beirne, S.; Shu, K.; Wallace, G. G. Electrochemistry Communications Three Dimensional ( $3 D$ ) Printed Electrodes for Interdigitated Supercapacitors. Electrochem. commun. 2014, 41, 20-23.

(11) Symes, M. D.; Kitson, P. J.; Yan, J.; Richmond, C. J.; Cooper, G. J. T.; Bowman, R. W.; Vilbrandt, T.; Cronin, L. Integrated 3D-Printed Reactionware for Chemical Synthesis and Analysis Mark. Nat. Chem. 2012, 4 (5), 349-354.

(12) Ambrosi, A.; Pumera, M. 3D-Printing Technologies for Electrochemical Applications. Chem. Soc. Rev. 2016, 45 (10), 2740-2755.

(13) Geissler, B. M.; Xia, Y. Patterning: Principles and Some New Developments. 2004, No. 15, 1249-1269.

(14) Trikantzopoulos, E.; Yang, C.; Ganesana, M.; Wang, Y.; Venton, B. J. Novel Carbon-Fiber Microelectrode Batch Fabrication Using a 3D-Printed Mold and Polyimide Resin. Analyst 2016, 3-7.

(15) Yang, C.; Denno, M. E.; Pyakurel, P.; Venton, B. J. Recent Trends in Carbon NanomaterialBased Electrochemical Sensors for Biomolecules: A Review. Anal. Chim. Acta 2015, 887, 17-37.

(16) Ping, J.; Wu, J.; Wang, Y.; Ying, Y. Simultaneous Determination of Ascorbic Acid, Dopamine and Uric Acid Using High-Performance Screen-Printed Graphene Electrode. Biosens. Bioelectron. 2012, 34 (1), 70-76.

(17) Moussa, M.; El-kady, M. F.; Wang, H.; Chervin, C. N.; Parker, J. F.; Nelson, E. S.; Rolison, D. R.; Long, J. W. CAD / CAM - Designed 3D-Printed Electroanalytical Cell for the Evaluation of Nanostructured Gas-Diffusion Electrodes. Nanotechnology 27 (17), 0.

(18) Amin, R.; Knowlton, S.; Hart, A.; Piqué, A.; Auyeung, R. C. Y.; Kim, H.; Bishop, G. W.; Satterwhite-warden, J. E. 3D-Printed Bioanalytical Devices. Nanotechnology 27 (28), 1-8.

(19) Miller, P. R.; Xiao, X.; Brener, I.; Burckel, D. B.; Narayan, R. Microneedle-Based Transdermal Sensor for On-Chip Potentiometric Determination of K +. 2014, 876-881. 
(20) Chen, J.-C.; Chung, H.-H.; Hsu, C.-T.; Tsai, D.-M.; Kumar, A. S.; Zen, J.-M. A Disposable Single-Use Electrochemical Sensor for the Detection of Uric Acid in Human Whole Blood. Sensors Actuators B 2005, 110, 364-369.

(21) Vilatela, J. J.; Marcilla, R. Tough Electrodes: Carbon Nanotube Fibers as the Ultimate Current Collectors/Active Material for Energy Management Devices. Chem. Mater. 2015, $27(20), 6901-6917$.

(22) Lu, W.; Zu, M.; Byun, J. H.; Kim, B. S.; Chou, T. W. State of the Art of Carbon Nanotube Fibers: Opportunities and Challenges. Adv. Mater. 2012, 24 (14), 1805-1833.

(23) Behabtu, N.; Green, M. J.; Pasquali, M. Carbon Nanotube-Based Neat Fibers. Nano Today 2008, 3 (5-6), 24-34.

(24) Harreither, W.; Trouillon, R.; Poulin, P.; Neri, W.; Ewing, A. G.; Safina, G. Carbon Nanotube Fiber Microelectrodes Show a Higher Resistance to Dopamine Fouling. Anal. Chem. 2013, 85 (15), 7447-7453.

(25) Yang, C.; Jacobs, C. B.; Nguyen, M. D.; Ganesana, M.; Zestos, A. G.; Ivanov, I. N.; Puretzky, A. A.; Rouleau, C. M.; Geohegan, D. B.; Venton, B. J. Carbon Nanotubes Grown on Metal Microelectrodes for the Detection of Dopamine. Anal. Chem. 2016, 88 (1), 645652.

(26) Schmidt, A. C.; Wang, X.; Zhu, Y.; Sombers, L. a. Carbon Nanotube Yarn Electrodes for Enhanced Detection of Neurotransmitter Dynamics in Live Brain Tissue. ACS Nano 2013, 7864-7873.

(27) Jacobs, C. B.; Ivanov, I. N.; Nguyen, M. D.; Zestos, A. G.; Venton, B. J. High Temporal Resolution Measurements of Dopamine with Carbon Nanotube Yarn Microelectrodes. Anal. Chem. 2014, 86 (12), 5721-5727.

(28) Dumitrescu, I.; Unwin, P. R.; Macpherson, J. V. Electrochemistry at Carbon Nanotubes: Perspective and Issues. Chem. Commun. (Camb). 2009, 7345 (45), 6886-6901.

(29) Kim, J.; Xiong, H.; Hofmann, M.; Kong, J.; Amemiya, S. Letters to Analytical Chemistry Scanning Electrochemical Microscopy of Individual Single-Walled Carbon Nanotubes. 2010, 82 (5), 1605-1607.

(30) Byers, J. C.; Güell, A. G.; Unwin, P. R. Nanoscale Electrocatalysis: Visualizing Oxygen Reduction at Pristine, Kinked, and Oxidized Sites on Individual Carbon Nanotubes. J. Am. Chem. Soc. 2014, 136 (32), 11252-11255.

(31) Güell, A. G.; Meadows, K. E.; Dudin, P. V.; Ebejer, N.; Macpherson, J. V.; Unwin, P. R. Mapping Nanoscale Electrochemistry of Individual Single-Walled Carbon Nanotubes. Nano Lett. 2014, 14 (1), 220-224.

(32) Mazloum-Ardakani, M.; Beitollahi, H.; Ganjipour, B.; Naeimi, H.; Nejati, M. Electrochemical and Catalytic Investigations of Dopamine and Uric Acid by Modified Carbon Nanotube Paste Electrode. Bioelectrochemistry 2009, 75 (1), 1-8.

(33) Banerjee, I.; Pangule, R. C.; Kane, R. S. Antifouling Coatings: Recent Developments in the Design of Surfaces That Prevent Fouling by Proteins, Bacteria, and Marine Organisms. Adv. Mater. 2011, 23 (6), 690-718.

(34) Zestos, A. G.; Jacobs, C. B.; Trikantzopoulos, E.; Ross, A. E.; Venton, B. J. Polyethylenimine Carbon Nanotube Fiber Electrodes for Enhanced Detection of Neurotransmitters. Anal. Chem. 2014, 86, 8568-8575.

(35) Yang, C.; Trikantzopoulos, E.; Nguyen, M. D.; Jacobs, C. B.; Wang, Y.; Mahjouri-Samani, M.; Ivanov, I. N.; Venton, B. J. Laser Treated Carbon Nanotube Yarn Microelectrodes for Rapid and Sensitive Detection of Dopamine in Vivo. ACS Sensors 2016, 1 (5), 508-515.

(36) Cao, M.; Fu, A.; Wang, Z.; Liu, J.; Kong, N.; Zong, X.; Liu, H.; Gooding, J. J. Electrochemical and Theoretical Study of $\Pi-\Pi$ Stacking Interactions between Graphitic Surfaces and Pyrene Derivatives. J. Phys. Chem. C 2014, 118 (5), 2650-2659.

(37) Hočevar, S. B.; Wang, J.; Deo, R. P.; Musameh, M.; Ogorevc, B. Carbon Nanotube Modified Microelectrode for Enhanced Voltammetric Detection of Dopamine in the 
Presence of Ascorbate. Electroanalysis 2005, 17 (5-6), 417-422.

(38) Swamy, B. E. K.; Venton, B. J. Carbon Nanotube-Modified Microelectrodes for Simultaneous Detection of Dopamine and Serotonin in Vivo. Analyst 2007, 132 (9), 876884.

(39) Takmakov, P.; Zachek, M. K.; Keithley, R. B.; Walsh, P. L.; Donley, C.; McCarty, G. S.; Wightman, R. M. Carbon Microelectrodes with a Renewable Surface. Anal. Chem. 2010, 82 (5), 2020-2028.

(40) Xiao, N.; Venton, B. Rapid, Sensitive Detection of Neurotransmitters at Microelectrodes Modified with Self-Assembled SWCNT Forests. Anal. Chem. 2012, 84 (18), 7816-7822.

(41) McCreery, R. L. Advanced Carbon Electrode Materials for Molecular Electrochemistry. Chem. Rev. 2008, 108 (7), 2646-2687.

(42) Venton, B. J.; Troyer, K. P.; Wightman, R. M. Response Times of Carbon Fiber Microelectrodes to Dynamic Changes in Catecholamine Concentration. Anal. Chem. 2002, 74 (3), 539-546.

(43) Muguruma, H.; Inoue, Y.; Inoue, H.; Ohsawa, T. Electrochemical Study of Dopamine at Electrode Fabricated by Cellulose-Assisted Aqueous Dispersion of Long-Length Carbon Nanotube. J. Phys. Chem. C 2016, 120 (22), 12284-12292.

(44) Hayat, A.; Marty, J. L. Disposable Screen Printed Electrochemical Sensors: Tools for Environmental Monitoring. Sensors (Switzerland) 2014, 14 (6), 10432-10453.

(45) Li, M.; Li, Y. T.; Li, D. W.; Long, Y. T. Recent Developments and Applications of ScreenPrinted Electrodes in Environmental Assays-A Review. Anal. Chim. Acta 2012, 734, 3144. 
Chapter 8: Conclusions and Future Directions 


\subsection{Overview}

This thesis has explored the synthesis and fabrication of several novel carbon nanomaterials, the effect of different surface modifications, and their applications for in vivo neurotransmitter detection. Moreover, the electrode surface properties were also characterized and then correlated to their electrochemical performance. The systematic comparison of different carbon nanomaterials and different surface modifications provides a useful structure to evaluate which new nanomaterials would be good as electrochemical sensor and which electrode a neuroscientist might choose for different experiments.

\subsubsection{Evaluation of Novel Carbon Nanomaterials and Different Surface Modifications for in vivo} Neurotransmitter Monitoring

Previous chapters introduced and discussed several successful synthesis or fabrication of novel carbon nanomaterials into microelectrodes and investigated their applications for the in vivo detection of neurotransmitters. One strategy is to directly grown carbon nanomaterials on metal substrates. Metal wires were used as substrates due to their high conductivity and electrochemical stability. Moreover, cylindrical metal substrates with a diameter of $25 \mu \mathrm{m}$ were used to reduce tissue damage and improve high spatial resolution. In Chapter 3, CNTs-metal microelectrodes were introduced and applied for in vivo dopamine detection. And recently CNS as a novel carbon nanomaterial has been grown on metal wires for electrochemical sensing as well. ${ }^{1}$

An alternative strategy is to fabricate CNT fibers and CNT yarns, which can be manufactured into microelectrodes easily and efficiently with high reproducibility. In chapter 6 , CNT yarns made by solid state process and CNT fiber made by wet spinning were compared, and CA/CNT fiber was used for biomolecule sensing for the first time. To further improve electrochemical performance on CNT yarn, three different surface modification methods, laser 
etching, $\mathrm{O}_{2}$ plasma etching, and anti-static gun treatment were introduced, as discussed in Chapter 3 and Chapter 4. Improved sensitivity, electron transfer rate, and fouling resistance were observed after surface modifications. In addition, different modification methods could specifically tune electrochemistry of CNT yarn, which provide a platform for fundamental electrochemistry study on carbon nanomaterials surface.

\subsubsection{Correlation of Surface Properties to Electrochemical Performance for Neurotransmitters} Detection

In the past decades, an increasing amount of articles using CNTs, graphene, or other carbon nanomaterials based microelectrodes for neurotransmitter detection have been published. Focusing solely on the detection of dopamine, which is one of the most common neurotransmitter detected by electrochemical means, a survey of the literature (Figure 8.1) indicates a clear rising trend of the number of publications for dopamine analysis using voltammetry methods at CNT and graphene based electrodes from 2006 to 2015 (results obtained of Web of Science). However, there are only very few works that truly compare different carbon nanomaterials and examine the reason why different carbon nanomaterials are good for the detection of neurotransmitters. The comparisons under the same condition would benefit proper choice of carbon nanomaterials or surface modifications for the detection of different neurotransmitters. This thesis also provides several comparison works of carbon nanomaterials for their electrochemical sensing abilities, filling the void of lack of fundamental studies comparing materials and their properties. 


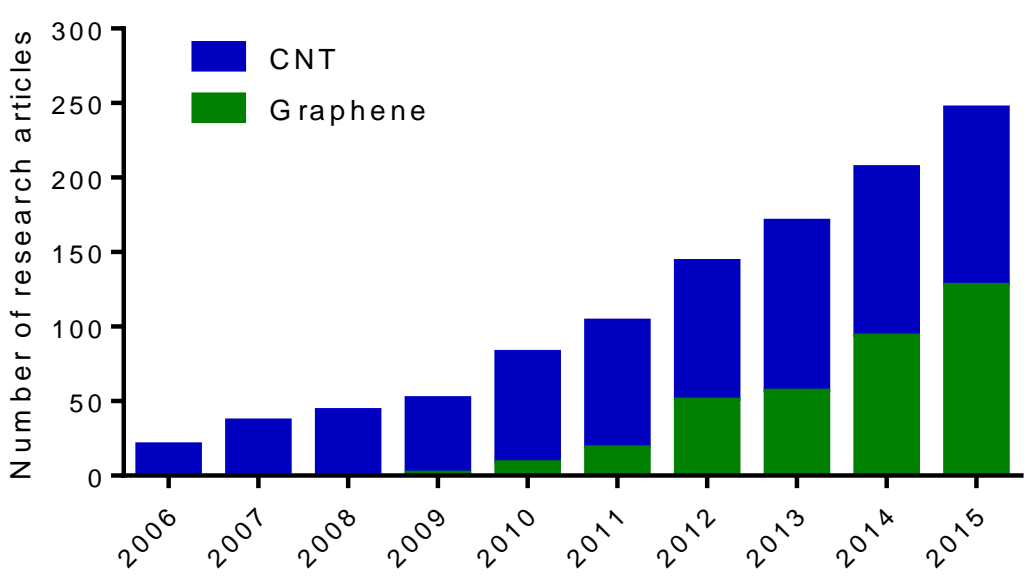

Figure 8.1: Number of publications for dopamine analysis using CNT and graphene based electrochemical methods (from 2006 to 2015). Results obtained of Web of Science ${ }^{\text {tm }}$.

\subsubsection{D Printing Technology Assisted Carbon Electrode Fabrication and the Application for} Electrochemical Sensing

The incorporation of 3D printing and electrochemical sensing applications would not only provide bespoke design and reduce the cost, but also provide an approach to fabricate electrodes with materials that could not be easily fabricated with traditional pulling methods. Chapter 6 and 7 provide two methods to use 3D printing technology for easy and low-cost carbon material electrodes for electrochemical detection of biomolecules. 3D printing technology was used to fabricate either a mold or an electrode substrate for carbon material- based electrode fabrication. With two early efforts using 3D printing-assisted electrode fabrication for the electrochemical sensing of dopamine, this thesis supports paths for future endeavors to take advantage of 3D printing. Although the hundreds-micron scale resolution is still a limitation, 3D printing technology is improving both in resolution and in the materials that can be printed. ${ }^{2-4}$ Thus, the direct printing of carbon based microelectrodes could possibly be achieved in the future with the deployment of 3D printing technology and nanometer scale printing resolution could be realized. 


\subsection{Future Directions}

\subsubsection{Short-Term Vision}

\subsubsection{Cylindrical CNT Yarn Microelectrode Fabrication Using Ion Polishing Method}

As mentioned in Chapter 3, 4 and 5, CNTs yarn microelectrodes are expected to be one of the best potential alternative to CFMEs for in vivo neurotransmitter detection because of several critical advantages including alignment, high conductivity, high chemical stability, promising fouling resistance, and the easy batch fabrication. Moreover, the electrochemical properties of CNT yarn microelectrodes can be controlled by surface treatments, as discussed in Chapter 3 and 4, which allows researchers to tune the material depending on the requirements of sensitivity and selectivity to different analytes. However, since CNT yarns are a soft material, cutting it with scalpel, which is used for carbon fibers, will not work: the end of the yarn will puff out after cutting so the geometry and homogeneity are changed. Therefore, all the applications of CNT yarns are limited to disk electrodes. The disk geometry, however, restricts the overall surface area and thus limits the sensitivity. Although our recent work shows several surface modification methods, such as anti-static gun or laser etching, can be applied to increase the surface roughness, the surface area is still significantly smaller than cylindrical carbon-fiber electrodes. So a new fabrication method for cylinder CNT yarn microelectrodes is needed to increase surface area and the overall sensitivity.

Focused ion beam milling (FIB) is a technique commonly used to cut samples for further SEM or TEM imaging of a cross section view. ${ }^{5}$ It could be used to cut CNT yarn with a smooth cut surface, however, FIB is not suitable for batch fabrication due to its intrinsically low milling rate and sample loading method. In comparison, ion polishing, often called ion milling, is a promising alternative method to cut CNT yarn with smooth surface. It could be used to fabricate as many as $30-40$ yarns simultaneously. lon polishing thins samples by firing ions (argon is commonly used) at the surface from an angle and sputtering material from the surface. Since the CNT yarn is thin, 
milling at a certain angle could easily cut batch of yarns at the middle and leave a smooth surface, as shown in Figure 8.2. With the help from Richard Wight in the Department of Material Science and Engineering at University of Virginia, we successfully cut more than ten CNT yarns the same time with smooth surface, proving the ion polishing method could be used for cylindrical CNTYMEs fabrication in the future. Although the surface is tilted and not perfectly flat (which would require further optimization), the preliminary data prove the feasibility to cut cylinder CNT yarns.

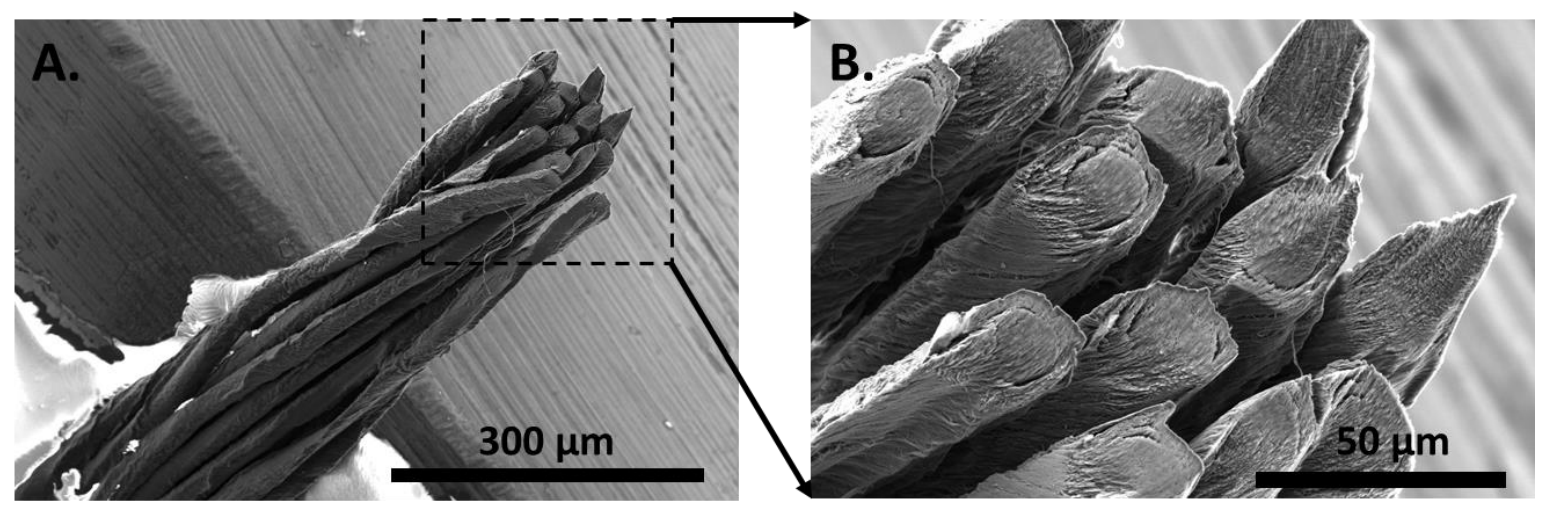

Figure 8.2: SEM images of ion polished CNT yarns. Scale bar: (A) $300 \mu \mathrm{m}$ and (B) $50 \mu \mathrm{m}$.

\subsubsection{Effect of Surface Functionality on Electrochemical Performance}

In the previous Chapters, we introduced that neurotransmitter detection, especially the detection of dopamine, a cation under physiological $\mathrm{pH}$, is dependent on the surface oxygencontaining functional groups. The surface oxide groups, such as quinones, hydroxyls, and carboxylic acids, are known to be negatively charged at physiological $\mathrm{pH}$ and have electrostatic interactions with positively charged neurotransmitters such as dopamine. ${ }^{6}$ However, the effect of surface functionalities on these properties has not been characterized. Previously, Runnels et al. and Chen et al. used 2,4-dinitrophenylhydrazine/3,5-dinitrobenzoyl chloride and cyclohexane polishing methods to selectively block different oxide groups on electrode surface. ${ }^{7,8}$ However, 
the slower electron transfer rate caused by the coating and the complicated modification methods limit their reproducibility. We reported another method that using modified carbon-fiber microelectrodes (CFMEs) with three differently functionalized SWCNTs $\left(\mathrm{COOH}-\mathrm{CNT}, \mathrm{CONH}_{2^{-}}\right.$ CNT, and ODA-CNT) to investigate the effect of the three different groups on electrochemical performance to neurotransmitters. ${ }^{9}$ The indirect study on the effect of functionality on electrochemistry, however, only provide limited information compared to the in situ measurement on the electrode surface.

X-ray photoelectron spectroscopic (XPS) is an important analytical tool to probe the elemental composition and the chemical state of samples. ${ }^{10}$ XPS has been used to detect the surface functionality on carbon electrode previously, ${ }^{11-17}$ but limited by the spatial resolution (hundreds microns), the measurements were only performed on millimeter sized glassy carbon electrodes (GCE) or GCE coated with carbon nanomaterial paste. With the development of surface characterization techniques, the resolution is improved to several microns scale, enabling the measurement of the functionality on microelectrode, such as CFMEs or CNTYMEs. ${ }^{12}$ The surface functionality correlated to electrochemical performance would provide a profound understanding of the contribution of different surface oxide groups.

Simulation is another path could help predict the correlation of surface functionality and electrochemical detection of neurotransmitters. We have an ongoing collaboration with Dr. DuBay group at Department of Chemistry, University of Virginia, to build a novel model to simulate the neurotransmitter redox reaction at carbon nanomaterial surface. Recently, they are using an allatom model to simulate both diffusion and adsorption processes of dopamine on defect-rich graphene layer with the external electric field applied. Different from the commonly used simulation tools, the all-atom model they are using would give an unprecedented perspective of the kinetic process of neurotransmitter reaction on the surface instead of just thermodynamic studies. Most of simulation works focuses on the thermodynamics in which a steady state needs 
to be reached. However, with fast scan rate and rapid scanning repetition frequency, the system usually does not reach its steady state, and a dynamic simulation is necessary. Our future goal is to mimic the electrochemical reaction of different neurotransmitters (e.g. adenosine, serotonin, ascorbic acid, et al.) when FSCV applied. As FSCV is becoming the most popular electrochemical technique for in vivo measurements of electroactive neurotransmitters, this model would be a very useful tool for all neuroscientists using FSCV system.

\subsubsection{Long-Term Vision}

\subsubsection{Reusable Carbon Nanomaterial Based Electrodes}

$\mathrm{CN}$-based electrochemical sensor manufacturing methods are becoming less expensive, more reproducible, and faster for batch fabrication. However, most of the sensors with CNs are non-reusable and the fouling of analytes/interferences is irreversible, which limits the sensors sustainability and potentially increases the cost for each measurement. To fabricate reusable $\mathrm{CN}$ electrodes, $\mathrm{TiO}_{2}$ nanoparticles can be incorporated with carbon nanomaterials due to its surface self-cleaning property.

Photocatalysts such as $\mathrm{TiO}_{2}$ and $\mathrm{ZnO}$ can decompose organic molecules by generating oxygen radicals after ultraviolet irradiation without altering the surface morphology and structure. 18,19 Photocatalytic cleaning methods have therefore been tested for feasibility with $\mathrm{CN}$-based sophisticated sensors. ${ }^{20} \mathrm{TiO}_{2}$ NP-decorated $\mathrm{CN}$-based sensors could be cleaned simply by ultraviolet light irradiation and re-used indefinitely. Xu et al. used a sensor fabricated by controllable assembly of reduced graphene oxide (RGO) and $\mathrm{TiO}_{2}$ to form a sandwich structure, followed by deposition of Au NPs onto the RGO shell, in which the encapsulated $\mathrm{TiO}_{2}$ ensures an excellent photocatalytic cleaning property (Fig. 5B). ${ }^{21} 5-\mathrm{HT}$ was used as a model passivation molecule, which caused a signal drop by accumulation of a non-electrochemically active oxidation product on the electrode surface. Thirty min of UV light irradiation after passivation recovers the 
sensitivity to $90 \%$ (Fig. 5C). The application of this renewable microsensor for sensitive detection of nitric oxide release from cells demonstrated the photocatalytic cleaning of absorbed polymers, proteins, and cell culture medium. Another advantage of $\mathrm{TiO}_{2}$ is the negative charge at physiological pH, which would repel other negatively charged interferences such as uric acid and ascorbic acid, and enhance the attraction of cationic neurochemicals (dopamine, adenosine, etc.). ${ }^{22}$ The combination of photocatalyst and $\mathrm{CNs}$ provides a versatile and efficient way for sensitive and renewable sensors for neurotransmitters detection.

Research into reusable electrodes is just beginning and will lead to electrodes that can be used either multiple times in vivo or as detectors for separations or in microfluidic devices, where constant changing of the sensor is not possible. The magnetically entrapped SWCNTs electrodes did not lose sensitivity with 15 consecutive measurements in ferrocyanide solution but more testing is needed for other interferences and surface fouling agents. ${ }^{23,24}$ In comparison, the RGO and $\mathrm{TiO}_{2} \mathrm{NP}$ electrodes lose some sensitivity over time, but the surface can be cleaned with UV light treatment. ${ }^{21}$ However, UV light treatment cannot be performed in the brain so this would only clean electrodes between samples. For the neurotransmitter monitoring at molecularly imprinted electrodes, the measurement is not real time (30 s - 10 min dopamine adsorption time was required), which limit the future applications. Overall, because of the large surface area, high conductivity of $\mathrm{CNs}$, the self-cleaning property, $\mathrm{TiO}_{2} / \mathrm{CNs}$ electrodes is the most favorable strategy for making reusable electrodes.

Although CNTs are known to have promising anti-fouling property, ${ }^{25}$ it has been reported the fouling on CNTs surface would significantly affect their electrochemical characteristics. ${ }^{26}$ Our recent findings on CNT yarn also indicate the remarkable fouling after placing electrode in tissue for 2 hours (Chapter 4). Therefore, the self-surface cleaning property of $\mathrm{TiO}_{2} \mathrm{NPs}$ when exposed to UV light is in need to enable electrodes to be re-used. In the near future, we can incorporate 
$\mathrm{TiO}_{2}$ NPs on our CNTYMEs or other carbon nanomaterial based electrodes to make microelectrodes reusable.

\subsubsection{Carbon Nanospike-Decorated Flexible Nano and Microsensors}

The development of customizable and flexible electrodes is another future path since most electrodes/arrays are made from stiff silicon or metal substrates, which limit their in vivo application. Traditional stiff devices produces a strong tissue reaction with implantation, resulting in the electrodes being encapsulated by fibrous tissue: this has the effect of electrically isolating the implant from the target tissue and reduces implant efficacy. ${ }^{27,28}$ The tissue reaction is mainly caused by the trauma due to sensor insertion, but recent studies have shown the flexible implants have a smaller tissue reaction effect, which could better protect implant functionality. ${ }^{29}$ For example, VanDersarl et al. fabricated a flexible implantable neural probe for electrochemical dopamine sensing. ${ }^{28}$ The electrode arrays were fabricated on silicon carrier wafers and then released from the substrate by chemical/electrochemical dissolution, which makes the electrodes array flexible and used for long-term neurotransmitter monitoring in vivo. However, the glassy carbon limits the detection sensitivity. Moreover, the sensors fabricated by normal lithograph methods or 3D printing have the drawback of millimeter scaled dimension, which restricts their future in vivo applications.

A promising 3D microfabrication method that has recently attracted considerable attention is two-photon polymerization based direct writing with ultrashort laser pulses. ${ }^{3,30}$ When focused into the volume of a photosensitive material, such as photoresist, the laser pulses initiate two-photon absorption and sub-sequent polymerization. This allows fabrication of any computer-generated 3D structure. More importantly, because of the threshold behavior and nonlinear nature of the process, a resolution beyond the diffraction limit can be realized, which can be as small as several nanometers, making the two-photon polymerization based laser direct writing technique a 
promising approach to minimize sensors dimension for in vivo biomarker measurement. For example, Miller et al. reported a microneedle based transdermal sensor for the potentiometric determination of potassium ions. ${ }^{31}$ The ion selective transdermal microneedle sensor is demonstrated for potassium by integrating 3D porous carbon and 3D porous graphene with a hollow microneedle, which was flexible, fully customized, and prepared using a laser direct write system utilizing two-photon polymerization. Therefore, two-photon polymerization based laser direct writing has the potential to fabricate $3 \mathrm{D}$ nano- and micro-scaled architectures with customizable geometry feasible for long-term neurotransmitter detection in vivo.

One limitation of the sensors is that the architecture itself is not conductive and not electroactive to neurotransmitters. Therefore, carbon or metal coating is necessary to give the nano- and micrometer scaled sensor biosensing ability. Since the high temperature coating methods, such as pyrolysis, could shrink the size of photoresist-made flexible substrates, a lowtemperature and low-pressure carbon nanomaterial fabrication method is desired. In 2015, we found that homogeneous coverage of substrate wires with complex arrays of catalyst-free graphene-like nanostructures, named carbon nanospikes (CNS), is possible with plasma enhanced chemical vapor deposition (PE-CVD). The CNS covered tantalum microelectrodes have enhanced sensitivity and selectivity to dopamine detection. ${ }^{1}$ Different than pyrolysis, CNS growth by PECVD under low-temperature condition would not cause the architecture to shrink. Moreover, CNS growth does not need buffer layer or catalyst, making CNS promising material for homogeneous and thorough coating on the surface. Therefore, nano-scaled sensors with homogeneous CNS coating could enable neurotransmitters detection in vivo with high sensitivity, high spatial resolution, and minimal tissue damage. In the near future, we will optimize the fabrication parameters and evaluate the flexible microsensor coating with carbon nanospikes for neurotransmitters as a novel neurotransmitter sensor design using a high precision and resolution of Nanoscribe Pro GT laser lithography system at Oak Ridge National Laboratory. 


\subsubsection{Cavity Carbon Nanopipette for Neurotransmitters Detection}

Over the past few decades, nanoelectrodes have been developed for electrochemical sensing applications. ${ }^{32-37}$ Carbon electrodes are preferred for neurotransmitter applications because of their low cost, wide potential window, and good adsorption properties. ${ }^{25}$ And nanoscale carbon electrodes are useful for localized detection of neurotransmitters at the level of single cells, single vesicles, as well as single synapses. Small animal models such as Drosophila and zebrafish have attracted more attention recently due to their homologous neurotransmitters with mammals and easy, fast genetic manipulations. ${ }^{38,34,39}$ The small dimensions of the central nervous systems of Drosophila and zebrafish requires better spatial resolution and a small, dagger-like electrode than can penetrate through the tough glial sheath barrier with minimal tissue damage.

Normally used carbon fiber microelectrodes with the diameter of $7 \mu \mathrm{m}$ and length of 50 $100 \mu \mathrm{m}$ have limited spatial resolution for the neurotransmitter detection in small organism such as Drosophila central nerve system. Carbon nanofiber microelectrodes fabricated on large silicon chips have been developed for neurotransmitter detection, but the large dimensions and the geometry limit the implantation. ${ }^{32}$ Flame-etched carbon fibers encased in nanopipettes as finite conical nanoelectrodes have been fabricated with 50-200 nm tip diameter and 500-2000 nm shaft length for the detection of neurotransmitter. ${ }^{33}$ However, the nanoelectrodes need to be fabricated individually, which also restricts the reproducibility. Our group reported a small, robust,

and sensitive carbon nanopipettes for in vivo neurotransmitter detection in Drosophila brain. ${ }^{34}$ The carbon nanopipette electrodes (CNPEs) were fabricated by selectively depositing a carbon layer on the inside of a pulled-quartz capillary, followed by the chemical etching process to expose the carbon tip, with $\sim 250 \mathrm{~nm}$ diameter tips, and controllable lengths of exposed carbon, ranging from 5 to $175 \mu \mathrm{m}$. Since the CNPEs were batch-fabricated in a furnace, CNPEs are feasible for batch fabrication with promising reproducibility. The CNPEs were used to detect endogenous dopamine 
release in Drosophila larvae using optogenetics. However, although the exposed length is controllable, its dimension is still on the micrometer scale, which makes the 'nanoelectrode' a 'microelectrode' when taking all three dimensions scales into account. Therefore, a new carbon nanoelectrode with a better detection sensitivity as well as an improved spatial resolution is needed for future applications.

Recently, Michael Mirkin's group at City University of New York developed CNPEs with a different electrode geometry using the same CVD based synthesis process. Instead of an extended tip of CNPE, the novel CNPEs have a tip diameter of 5-100 nm with either a cavity near the orifice or with an open path in the middle, in which the volume of sampled solution is controlled by the applied pressure. Compared to the normal CNPEs with extended tip, the CNPEs with cavity or open tube have much smaller spatial resolution, which is the same as a disk electrodes (only depends on the disk diameter). Second, the cavity or open tube geometry provides a larger surface area inside the electrodes, which is also covered by carbon. Since the cavity could trap both dopamine and the oxidation product, dopamine-o-quinone (DOQ), the electrode would reach a thin-film condition which would help amplifying the signal.

We have a collaboration with Mirkin lab to apply their cavity or open tube geometry CNPEs to neurotransmitter detection using FSCV. Figure 8.3 shows preliminary data of the response to $10 \mu \mathrm{M}$ dopamine at a cavity CNPE electrode. Measurements were taken after 5 mins to 30 mins of equilibration in PBS buffer with a waveform of -0.4 to $1.3 \mathrm{~V}$ and back at $400 \mathrm{~V} / \mathrm{s}$, and scanning repetition frequency of $10 \mathrm{~Hz}$. The signal keeps increasing with increasing amount of equilibration time, and the equilibration is achieved by about 30 mins. Equilibration at the CNPEs is required due to two reasons. First, the open tube geometry requires an amount of time for the siphon effect to draw solution into the tip to a stable level. In addition, the waveform applied by FSCV mildly etches the carbon surface and introduces more surface oxide groups, which also needs time to achieve equilibration. ${ }^{12}$ Compared to the conventionally used CFMEs, the oxidation and reduction 
peaks are more symmetric, indicating a better dopamine redox reaction reversibility, which is consistent with signals obtained at a thin-film condition.
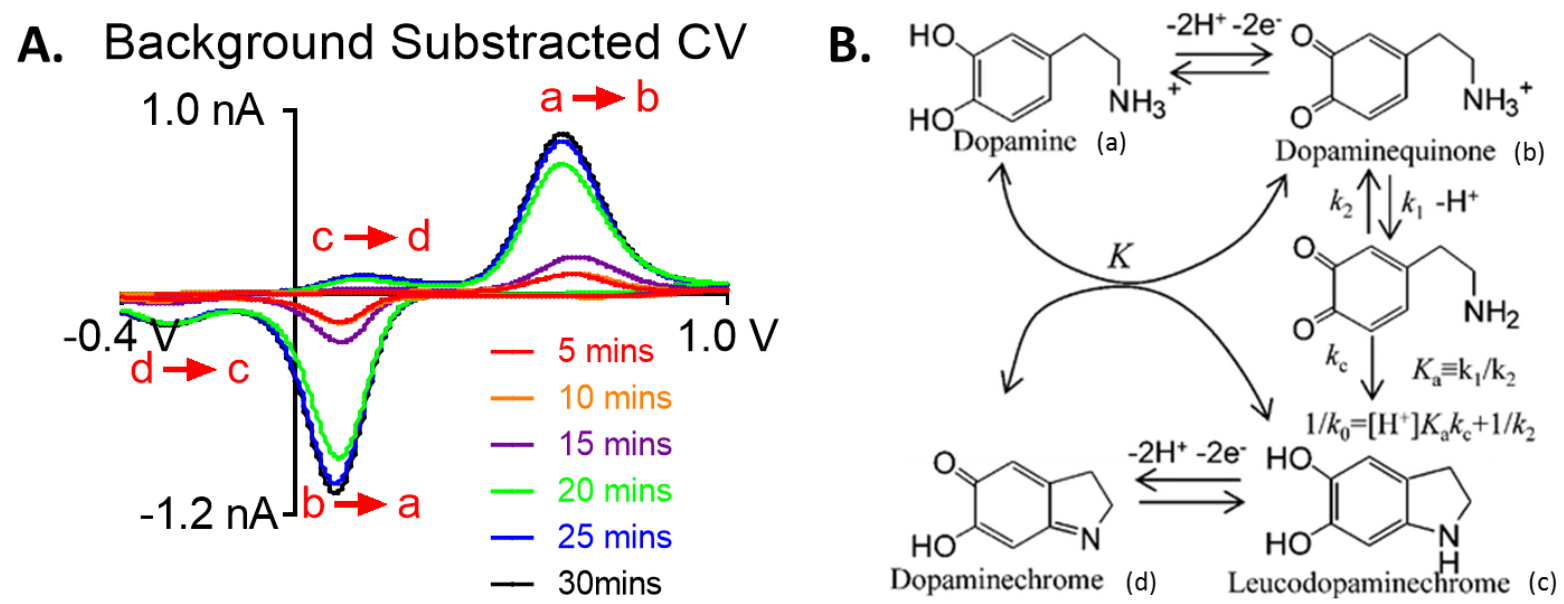

Figure 8.3: (A) CVs recorded at an open tube CNPE in $10 \mu \mathrm{M}$ dopamine PBS solution with equilibration time of 5 mins (red), 10 mins (orange), 15 mins (purple), 20 mins (green), 25 mins (blue), and 30 mins (black). Measurements were taken using FSCV with a waveform of -0.4 to $1.3 \mathrm{~V}$ and back at $400 \mathrm{~V} / \mathrm{s}$ and scanning repetition rate of $10 \mathrm{~Hz}$. (B) Dopamine oxidation scheme. Reprinted with permission from reference [42], Copyright 2016 American Chemical Society.

Notably, two redox pairs due to the reaction of dopamine are found with the open tube CNPEs. Dopamine undergoes a series of reactions, and the dopamine reaction pathway is summarized in Figure 8.3B: following the two-electron oxidation of dopamine (a, DA) to dopamineo-quinone (b, DOQ), ring closure via deprotonation of the amine side chain to leucodopaminechrome (c, LDAC) occurs irreversibly. And then compound $\mathrm{c}$ is oxidized to dopaminechrome $(\mathrm{d}, \mathrm{DAC})$. Although redox peaks due to $\mathrm{a} \rightleftharpoons \mathrm{b}$ and $\mathrm{c} \rightleftharpoons \mathrm{d}$ in dopamine analyses have been recognized previously, ${ }^{14,40,41}$ most researchers have not been interested in the $\mathrm{c} \rightleftharpoons \mathrm{d}$ reaction since it's not stable and the peak is broader than the peaks caused by reaction $a \rightleftharpoons b$. It has been reported that the rich and long m-electron network of the long-length CNT reduces an 
activation potential for chemical (oxidative) reaction and efficiently propagates the electron produced by $\mathrm{c} \rightarrow \mathrm{d}$ reaction. ${ }^{42}$ However, based on this preliminary data, another possibility might be due to the different morphology of carbon surface which could change dopamine diffusion profile: the longer time and higher concentration of DOQ versus DA remained on electrode surface would cause the second redox reaction happen. Therefore, theoretically, with faster scan rate using FSCV, an increased secondary oxidation peak of $\mathrm{c}$ to $\mathrm{d}$ could be obtained.

The preliminary data indicates the cavity and open tube CNPEs performs differently with the general CNPEs with an extruding tip. Future studies are still required to test the electrode stability and temporal resolution, which would be influenced significantly caused by the thin-film condition. 


\subsection{Final Remarks}

Overall, this dissertation described several novel carbon nanomaterial based electrodes fabrication methods, investigated in vivo applications, and filled up the void of fundamental studies comparing materials and their properties. The field of carbon nanomaterial based electrodes is exploding dramatically in the past decade. However, most of recent researches focuses on utilizing a single type of carbon nanomaterials, and lacks a comparison of different carbon nanomaterials for biomolecules detection. Many studies have focused on sensor design and proof of principle detection, but only a few studies have been performed in real biological samples. My work answers both fundamental and practical considerations for implementation in real experiments focusing on neurotransmitters.

For the practical application of electrodes, we successfully applied novel carbon materials (CNT- metal, CNS - metal, CNT yarn, and CNT fibers made by different polymers), new surface treatments (laser etching, $\mathrm{O}_{2}$ plasma etching, and anti-static gun treatment), and advanced sensor fabrication methods (3D printed mold and 3D printed electrode substrate). In addition, the in vivo monitoring has been performed and investigated at most of novel carbon materials, electrodes modified with different surface treatments and fabricated using different manufacture approaches. For the fundamental studies, first, we endeavored for a better understanding of how surface properties lead to electrochemical properties using several methods (e.g., Langmuir adsorption isotherm and diffusion model for better understanding of dopamine redox reaction on electrode surface). A systematic comparison of different carbon nanomaterials and different surface modifications provides a useful structure to evaluate which new nanomaterials would be good as electrochemical sensor and which electrode a neuroscientist might choose for different experiments. Moreover, novel materials (e.g., carbon nanopipette) and electrode fabrication method (e.g., nano-scale printed electrodes) further provide tools for better understanding of fundamental electrochemistry which cannot be achieved by other materials or conventionally 
fabrication methods. Therefore, these works described in this dissertation lead to better understanding and application of carbon nanomaterials sensors for neurotransmitters detection, which could facilitate the neuroscience study of behaviors and neurodegenerative disease and promise to be a fruitful research area for years to come. 


\subsection{References}

(1) Zestos, A. G.; Yang, C.; Jacobs, C. B.; Hensley, D.; Venton, B. J. Carbon Nanospikes Grown on Metal Wires as Microelectrode Sensors for Dopamine. Analyst 2015, 140 (21), 7283-7292.

(2) Amin, R.; Knowlton, S.; Hart, A.; Piqué, A.; Auyeung, R. C. Y.; Kim, H.; Bishop, G. W.; Satterwhitewarden, J. E. 3D-Printed Bioanalytical Devices. Nanotechnology 27 (28), 1-8.

(3) Ambrosi, A.; Pumera, M. 3D-Printing Technologies for Electrochemical Applications. Chem. Soc. Rev. 2016, 45 (10), 2740-2755.

(4) Bak, D. Rapid Prototyping or Rapid Production? 3D Printing Processes Move Industry towards the Latter. Assem. Autom. 2003, 23 (4), 340-345.

(5) Giannuzzi, L. A.; Stevie, F. A. A Review of Focused Ion Beam Milling Techniques for TEM Specimen Preparation. Micron 1999, 30 (3), 197-204.

(6) Roberts, J. G.; Moody, B. P.; McCarty, G. S.; Sombers, L. a. Specific Oxygen-Containing Functional Groups on the Carbon Surface Underlie an Enhanced Sensitivity to Dopamine at Electrochemically Pretreated Carbon Fiber Microelectrodes. Langmuir 2010, 26 (11), 9116-9122.

(7) Runnels, P. L.; Joseph, J. D.; Logman, M. J.; Wightman, R. M. Effect of pH and Surface Functionalities on the Cyclic Voltammetric Responses of Carbon-Fiber Microelectrodes. Anal. Chem. 1999, 71 (14), 2782-2789.

(8) Chen, P.; McCreery, R. L. Control of Electron Transfer Kinetics at Glassy Carbon Electrodes by Specific Surface Modification. Anal. Chem. 1996, 68 (22), 3958-3965.

(9) Jacobs, C. B.; Vickrey, T. L.; Venton, B. J. Functional Groups Modulate the Sensitivity and Electron Transfer Kinetics of Neurochemicals at Carbon Nanotube Modified Microelectrodes. Analyst 2011, 136 (17), 3557.

(10) Filik, J.; May, P. W.; Pearce, S. R. J.; Wild, R. K.; Hallam, K. R. XPS and Laser Raman Analysis of Hydrogenated Amorphous Carbon Films. Diam. Relat. Mater. 2003, 12 (3-7), 974-978.

(11) Yang, J.; Zhang, Y.; Kim, D. Y. Electrochemical Sensing Performance of Nanodiamond-Derived Carbon Nano-Onions: Comparison with Multiwalled Carbon Nanotubes, Graphite Nanoflakes, and Glassy Carbon. Carbon N. Y. 2016, 98, 74-82.

(12) Takmakov, P.; Zachek, M. K.; Keithley, R. B.; Walsh, P. L.; Donley, C.; McCarty, G. S.; Wightman, R. M. Carbon Microelectrodes with a Renewable Surface. Anal. Chem. 2010, 82 (5), 2020-2028.

(13) Canevari, T. C.; Raymundo-Pereira, P. a; Landers, R.; Benvenutti, E. V; Machado, S. a S. Sol-Gel ThinFilm Based Mesoporous Silica and Carbon Nanotubes for the Determination of Dopamine, Uric Acid and Paracetamol in Urine. Talanta 2013, 116, 726-735.

(14) Sainio, S.; Palom??ki, T.; Rhode, S.; Kauppila, M.; Pitk??nen, O.; Selk??l??, T.; Toth, G.; Moram, M.; Kordas, K.; Koskinen, J.; Laurila, T. Carbon Nanotube (CNT) Forest Grown on Diamond-like Carbon (DLC) Thin Films Significantly Improves Electrochemical Sensitivity and Selectivity towards Dopamine. Sensors Actuators, B Chem. 2015, 211, 177-186.

(15) Jia, D.; Dai, J.; Yuan, H.; Lei, L.; Xiao, D. Selective Detection of Dopamine in the Presence of Uric Acid Using a Gold Nanoparticles-Poly(luminol) Hybrid Film and Multi-Walled Carbon Nanotubes with Incorporated $\beta$-Cyclodextrin Modified Glassy Carbon Electrode. Talanta 2011, 85 (5), 2344- 
2351.

(16) Li, F.; Chai, J.; Yang, H.; Han, D.; Niu, L. Synthesis of Pt/ionic Liquid/graphene Nanocomposite and Its Simultaneous Determination of Ascorbic Acid and Dopamine. Talanta 2010, 81 (3), 1063-1068.

(17) Huang, T.-C.; Yeh, L.-C.; Huang, H.-Y.; Nian, Z.-Y.; Yeh, Y.-C.; Chou, Y.-C.; Yeh, J.-M.; Tsai, M.-H. The Use of a Carbon Paste Electrode Mixed with Multiwalled Carbon Nanotube/electroactive Polyimide Composites as an Electrode for Sensing Ascorbic Acid. Polym. Chem. 2014, 5 (2), 630.

(18) Hisatomi, T.; Kubota, J.; Domen, K. Recent Advances in Semiconductors for Photocatalytic and Photoelectrochemical Water Splitting. Chem. Soc. Rev. 2014, 43 (22), 7520-7535.

(19) Wang, H.; Zhang, L.; Chen, Z.; Hu, J.; Li, S.; Wang, Z.; Liu, J.; Wang, X. Semiconductor Heterojunction Photocatalysts: Design, Construction, and Photocatalytic Performances. Chem. Soc. Rev. 2014, 43 (15), 5234-5244.

(20) Li, X.; Chen, G.; Yang, L.; Jin, Z.; Liu, J. Multifunctional Au-Coated TiO2 Nanotube Arrays as Recyclable SERS Substrates for Multifold Organic Pollutants Detection. Adv. Funct. Mater. 2010, 20 (17), 2815-2824.

(21) Xu, J.-Q.; Liu, Y.-L.; Wang, Q.; Duo, H.-H.; Zhang, X.-W.; Li, Y.-T.; Huang, W.-H. Photocatalytically Renewable Micro-Electrochemical Sensor for Real-Time Monitoring of Cells. Angew. Chemie Int. Ed. 2015, 54 (48), 14402-14406.

(22) Pifferi, V.; Soliveri, G.; Panzarasa, G.; Cappelletti, G.; Meroni, D.; Falciola, L. Photo-Renewable Electroanalytical Sensor for Neurotransmitters Detection in Body Fluid Mimics. Anal. Bioanal. Chem. 2016.

(23) Baldrich, E.; Gómez, R.; Gabriel, G.; Muñoz, F. X. Magnetic Entrapment for Fast, Simple and Reversible Electrode Modification with Carbon Nanotubes: Application to Dopamine Detection. Biosens. Bioelectron. 2011, 26 (5), 1876-1882.

(24) Herrasti, Z.; Martínez, F.; Baldrich, E. Detection of Uric Acid at Reversibly Nanostructured Thin-Film Microelectrodes. Sensors Actuators B Chem. 2016, 234, 667-673.

(25) Yang, C.; Denno, M. E.; Pyakurel, P.; Venton, B. J. Recent Trends in Carbon Nanomaterial-Based Electrochemical Sensors for Biomolecules: A Review. Anal. Chim. Acta 2015, 887, 17-37.

(26) Liu, X.; Zhang, M.; Xiao, T.; Hao, J.; Li, R.; Mao, L. Protein Pretreatment of Microelectrodes Enables in Vivo Electrochemical Measurements with Easy Precalibration and Interference-Free from Proteins. Anal. Chem. 2016, 88 (14), 7238-7244.

(27) Mercanzini, A.; Colin, P.; Bensadoun, J.-C.; Bertsch, A.; Renaud, P. In Vivo Electrical Impedance Spectroscopy of Tissue Reaction to Microelectrode Arrays. IEEE Trans. Biomed. Eng. 2009, 56 (7), 1909-1918.

(28) Van Dersarl, J. J.; Mercanzini, A.; Renaud, P. Integration of 2D and 3D Thin Film Glassy Carbon Electrode Arrays for Electrochemical Dopamine Sensing in Flexible Neuroelectronic Implants. Adv. Funct. Mater. 2015, 25 (1), 78-84.

(29) Mercanzini, A.; Cheung, K.; Buhl, D.; Boers, M.; Maillard, A.; Colin, P.; Bensadoun, J.-C.; Bertsch, A.; Carleton, A.; Renaud, P. Demonstration of Cortical Recording and Reduced Inflammatory Response Using Flexible Polymer Neural Probes. 2007 IEEE 20th Int. Conf. Micro Electro Mech. Syst. 2007, No. 
January, 573-576.

(30) Therriault, D.; White, S. R.; Lewis, J. A. Chaotic Mixing in Three-Dimensional Microvascular Networks Fabricated by Direct-Write Assembly. 2003, 2 (April), 265-272.

(31) Miller, P. R.; Xiao, X.; Brener, I.; Burckel, D. B.; Narayan, R. Microneedle-Based Transdermal Sensor for On-Chip Potentiometric Determination of K +. 2014, 876-881.

(32) Koehne, J. E.; Marsh, M.; Boakye, A.; Douglas, B.; Kim, I. Y.; Chang, S.-Y.; Jang, D.-P.; Bennet, K. E.; Kimble, C.; Andrews, R.; Meyyappan, M.; Lee, K. H. Carbon Nanofiber Electrode Array for Electrochemical Detection of Dopamine Using Fast Scan Cyclic Voltammetry. Analyst 2011, 136 (9), 1802.

(33) Li, Y.; Zhang, S.-H.; Wang, L.; Xiao, R.; Liu, W.; Zhang, X.; Zhou, Z.; Amatore, C.; Huang, W. Nanoelectrode for Amperometric Monitoring of Individual Vesicular Exocytosis Inside Single Synapses. Angew. Chemie Int. Ed. 2014, 53 (46), 12456-12460.

(34) Rees, H. R.; Anderson, S. E.; Privman, E.; Bau, H. H.; Venton, B. J. Carbon Nanopipette Electrodes for Dopamine Detection in Drosophila. Anal. Chem. 2015, 87 (7), 3849-3855.

(35) Yu, Y.; Noël, J. M.; Mirkin, M. V.; Gao, Y.; Mashtalir, O.; Friedman, G.; Gogotsi, Y. Carbon PipetteBased Electrochemical Nanosampler. Anal. Chem. 2014, 86, 3365-3372.

(36) Arrigan, D. W. M. Nanoelectrodes, Nanoelectrode Arrays and Their Applications. Analyst 2004, 129 (12), 1157.

(37) Shen, M.; Colombo, M. L. Electrochemical Nanoprobes for the Chemical Detection of Neurotransmitters. Anal. Methods 2015, 7 (17), 7095-7105.

(38) Bucher, E. S.; Wightman, R. M. Electrochemical Analysis of Neurotransmitters. Annu. Rev. Anal. Chem. (Palo Alto. Calif). 2015, 8, 239-261.

(39) Traver, D.; Paw, B. H.; Poss, K. D.; Penberthy, W. T.; Lin, S.; Zon, L. I. Transplantation and in Vivo Imaging of Multilineage Engraftment in Zebrafish Bloodless Mutants. Nat. Immunol. 2003, 4 (12), 1238-1246.

(40) Manjunatha, R.; Suresh, G. S.; Melo, J. S.; D'Souza, S. F.; Venkatesha, T. V. Simultaneous Determination of Ascorbic Acid, Dopamine and Uric Acid Using Polystyrene Sulfonate Wrapped Multiwalled Carbon Nanotubes Bound to Graphite Electrode through Layer-by-Layer Technique. Sensors Actuators B Chem. 2010, 145 (2), 643-650.

(41) Li, Y.; Du, J.; Yang, J.; Liu, D.; Lu, X. Electrocatalytic Detection of Dopamine in the Presence of Ascorbic Acid and Uric Acid Using Single-Walled Carbon Nanotubes Modified Electrode. Colloids Surf. B. Biointerfaces 2012, 97 (2012), 32-36.

(42) Muguruma, H.; Inoue, Y.; Inoue, H.; Ohsawa, T. Electrochemical Study of Dopamine at Electrode Fabricated by Cellulose-Assisted Aqueous Dispersion of Long-Length Carbon Nanotube. J. Phys. Chem. C 2016, 120 (22), 12284-12292. 